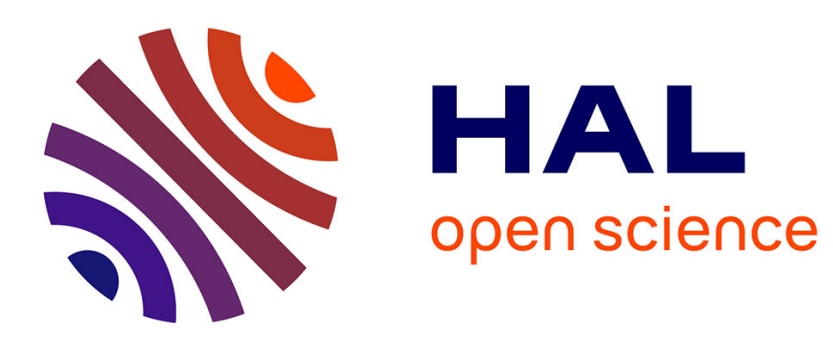

\title{
General Description of Fission Observables: GEF Model Code
}

\author{
Karl-Heinz Schmidt, Beatriz Jurado, Charlotte Amouroux, C. Schmitt
}

\section{To cite this version:}

Karl-Heinz Schmidt, Beatriz Jurado, Charlotte Amouroux, C. Schmitt. General Description of Fission Observables: GEF Model Code. Nuclear Data Sheets, 2016, 131, pp.107-221. 10.1016/j.nds.2015.12.009 . in2p3-01241907

\section{HAL Id: in2p3-01241907 https://hal.in2p3.fr/in2p3-01241907}

Submitted on 11 Dec 2015

HAL is a multi-disciplinary open access archive for the deposit and dissemination of scientific research documents, whether they are published or not. The documents may come from teaching and research institutions in France or abroad, or from public or private research centers.
L'archive ouverte pluridisciplinaire HAL, est destinée au dépôt et à la diffusion de documents scientifiques de niveau recherche, publiés ou non, émanant des établissements d'enseignement et de recherche français ou étrangers, des laboratoires publics ou privés. 


\title{
General Description of Fission Observables: GEF Model Code
}

\author{
Karl-Heinz Schmidt, ${ }^{1, *}$ Beatriz Jurado, ${ }^{1, \dagger}$ Charlotte Amouroux, ${ }^{2}$ and Christelle Schmitt ${ }^{3, \ddagger}$ \\ ${ }^{1}$ CENBG, CNRS/IN2 P3, Chemin du Solarium B.P. 120, F-33175 Gradignan, France \\ ${ }^{2} C E A, D S M-S a c l a y$, France \\ ${ }^{3}$ GANIL, Bd. Henri Becquerel, B.P. 55027, F-14076 Caen Cedex 05, France
}

(Dated: October 2, 2015; Received xx July 2015; revised received xx September 2015; accepted xx October 2015)

The GEF ("GEneral description of Fission observables") model code is documented. It describes the observables for spontaneous fission, neutron-induced fission and, more generally, for fission of a compound nucleus from any other entrance channel, with given excitation energy and angular momentum. The GEF model is applicable for a wide range of isotopes from $\mathrm{Z}=80$ to $\mathrm{Z}=112$ and beyond, up to excitation energies of about $100 \mathrm{MeV}$. The results of the GEF model are compared with fission barriers, fission probabilities, fission-fragment mass- and nuclide distributions, isomeric ratios, total kinetic energies, and prompt-neutron and prompt-gamma yields and energy spectra from neutron-induced and spontaneous fission. Derived properties of delayed neutrons and decay heat are also considered.

The GEF model is based on a general approach to nuclear fission that explains a great part of the complex appearance of fission observables on the basis of fundamental laws of physics and general properties of microscopic systems and mathematical objects. The topographic theorem is used to estimate the fission-barrier heights from theoretical macroscopic saddle-point and ground-state masses and experimental ground-state masses. Motivated by the theoretically predicted early localisation of nucleonic wave functions in a necked-in shape, the properties of the relevant fragment shells are extracted. These are used to determine the depths and the widths of the fission valleys corresponding to the different fission channels and to describe the fission-fragment distributions and deformations at scission by a statistical approach. A modified composite nuclear-level-density formula is proposed. It respects some features in the superfluid regime that are in accordance with new experimental findings and with theoretical expectations. These are a constant-temperature behaviour that is consistent with a considerably increased heat capacity and an increased pairing condensation energy that is consistent with the collective enhancement of the level density. The exchange of excitation energy and nucleons between the nascent fragments on the way from saddle to scission is estimated according to statistical mechanics. As a result, excitation energy and unpaired nucleons are predominantly transferred to the heavy fragment in the superfluid regime. This description reproduces some rather peculiar observed features of the prompt-neutron multiplicities and of the even-odd effect in fission-fragment $Z$ distributions. For completeness, some conventional descriptions are used for calculating pre-equilibrium emission, fission probabilities and statistical emission of neutrons and gamma radiation from the excited fragments. Preference is given to simple models that can also be applied to exotic nuclei compared to more sophisticated models that need precise empirical input of nuclear properties, e.g. spectroscopic information.

The approach reveals a high degree of regularity and provides a considerable insight into the physics of the fission process. Fission observables can be calculated with a precision that complies with the needs for applications in nuclear technology without specific adjustments to measured data of individual systems. The GEF executable runs out of the box with no need for entering any empirical data. This unique feature is of valuable importance, because the number of systems and energies of potential significance for fundamental and applied science will never be possible to be measured. The relevance of the approach for examining the consistency of experimental results and for evaluating nuclear data is demonstrated.

\footnotetext{
* Corresponding author: schmidt-erzhausen@t-online.de, URL: http://www.khs-erzhausen.de

$\dagger$ E-mail: jurado@cenbg.in2p3.fr

$\ddagger$ E-mail: schmitt@ganil.fr
} 


\section{CONTENTS}

I. Introduction
A. Core of the GEF Model
B. Additional Ingredients
C. Understanding of the Fission Process
D. Further Developments and Applications
E. Complementing, Estimating and Evaluating Nuclear Data

3

3

4

4

4

4

II. Basic Ideas of the GEF Model

A. Topological Properties of the Nuclear Potential in Multi-dimensional Space

B. Statistical Filling of Quantum Oscillators

C. Early Localization of Nucleonic Wave Functions

D. Characteristic Times of Stochastic Processes

E. Entropy-driven Energy Sorting

F. Exploiting Empirical Knowledge

G. Generality

H. Correlations and Dependencies

I. Range of Validity

III. Modeling of the Fission Process

A. Systematics of Fission Barriers

B. Nuclear Level Densities

C. Empirical Fragment Shells

D. Charge Polarization

E. Quantum Oscillators of Normal Modes

F. Energetics of the Fission Process

1. From Saddle to Scission

2. Fully Accelerated Fragments

G. Even-odd Effects

1. $Z$ Distribution

2. $N$ Distribution

3. Total Kinetic Energy

H. Spontaneous Fission

I. Fission-fragment Angular Momentum

J. Emission of Prompt Neutrons and Prompt Gammas

IV. Particle-induced Fission
A. Neutron-induced Fission
B. Charged-particle- and Heavy-ion-induced Fission

V. Multi-chance Fission

VI. Parameter Values
A. Positions of the Fission Channels
B. Widths of the Fission Channels
C. Strength of the Fragment Shells
D. Fragment Deformation
E. Charge Polarization

4

5

5

7

8

8

8

8

9

14

15

16

17

19

19

20

21

21

22

22

23

24

25

27

27

29

29

30

30

30

31

31

32

F. Energies and Temperatures

1. Temperatures

2. Excitation Energy at Scission

3. Deformation Energy

4. Tunneling

G. Concluding Remarks

VII. Computational Structure of tha GEF Code A. Flowchart of the GEF Code

1. Pre-fission Processes

2. Average Fission-fragment Properties

3. Monte-.Carlo Sampling of Fission Events

B. Subroutines

C. Input and Output

1. Input

2. Output

VIII. Uncertainties and Covariances

IX. Assessment

A. Fission Probability

1. Introduction

2. Formulation of the Fission Probability

3. Comparison with Experimental Data

4. Discussion

5. Conclusion

B. Fission-fragment Yields

1. Introduction

2. Experimental Technique;

3. Mass Distributions

4. Deviations

5. Charge Polarization and Emission of Prompt Neutrons

6. Nuclide Distributions

7. Energy Dependence

8. Discussion

9. Conclusion and Outlook

C. Isomeric Yields

1. Comparison with Measuced Data

2. Even-odd Staggering in Angular Momentum

D. Prompt-neutron Multiplicities

1. System Dependence

2. Energy Dependence

3. Fragment-mass Dependence

4. Multiplicity Distributions

5. Conclusion

E. Prompt-neutron Energies

1. Key Systems

2. Energy Dependence

F. Prompt-gamma Emission

1. Average Values

2. Energy Spectrum

3. Saw-tooth Gamma Multiplicity

G. Fragment Kinetic Energies

X. Data for Application 

A. Decay Heat
B. Delayed Neutrons

99

XI. Validation and Evaluation of Nuclear Data

101

A. Indications for a Target Contaminant

102

B. An Inconsistent Mass Identification

C. Complex Properties of Fission Channels

D. Data Completion and Evaluation

1. Mathematical Procedure in Two Dimensions

2. Two Examples

102

103

103

103

104

XII. Conclusion

Acknowledgments

References

\section{INTRODUCTION}

This work is an updated and extended version of a recent documentation of the GEF model in the JEFF Report 24 [1, 2] of the NEA Data Bank.

\section{A. Core of the GEF Model}

The phenomena related to nuclear fission result from many different processes. For many of these, there exist very elaborate models, for example for the capture of an incoming particle in a target nucleus and for the emission of neutrons and gamma radiation from an excited nucleus. However, the modeling of the re-ordering of the nucleons from an excited mono-nucleus into two (or eventually more) fragments is still a challenge for nuclear theory. Estimating the properties of the fission fragments with the high quality required for applications in nuclear technology still relies on empirical models [3]. The excitation energy of the primary fragments gives rise to promptneutron and prompt-gamma emission. The composition of the fission products in $A$ and $Z$ after prompt-neutron emission determines the starting points of the radioactive decay chains and defines the decay-heat production. The major problem is that a fissioning nucleus is an open system that evolves from a quasi-bound configuration to a continuum of possible configurations on the fission path, finally forming hundreds of different fragments with continuous distributions of different shapes, kinetic energies, excitation energies and angular momenta. One of the most advanced approaches for modeling low-energy nuclear fission describes the fission process by a numerical solution of the Langevin equations [4 [6]. A random-walk approach that neglects the influence of inertia on the nuclear dynamics has been proposed in Ref. 77. In these stochastic models, a subspace of collective variables that is restricted by the limited available computing power is explicitly considered, while all other collective and intrin- sic degrees of freedom are replaced by a heat bath. It is a draw-back that quantum-mechanical features are not properly considered in this classical approach. Another approach follows the evolution of the fissioning system with quantum-mechanical tools [8]. However, the inclusion of dissipative processes and phenomena of statistical mechanics within quantum-mechanical algorithms is still not sufficiently developed [9, 10]. Also these calculations require very large computer resources.

The GEneral description of Fission observables (GEF model) presented in this work makes use of many theoretical ideas of mostly rather general character, avoiding microscopic calculations with their approximations and limitations, e.g. by the high computational needs. The large body of empirical information is used for developing a global description of the fission quantities, which is in good agreement with the empirical data. The theoretical frame assures that this model is able to provide quantitative predictions of the manifold fission observables for a wide range of fissioning systems. The model has a high degree of generality. Firstly, it is applicable to essentially all fissioning systems from spontaneous fission up to excitation energies of about $100 \mathrm{MeV}$ without specific adjustment to individual fissioning systems, secondly, it calculates the majority of all possible fission quantities, and, thirdly, it is based on general properties of microscopic systems and on general properties of a function in multidimensional space.

The empirical input to the model required adjusting a number of parameters by performing a large number of calculations for many systems. For this purpose, it is important that the calculation is relatively fast, allowing for applying fit procedures in order to find the optimum parameter values.

The relationship between GEF and microscopic fission models may best be illustrated by recalling the role of the liquid-drop model in the development of nuclear mass models, although the dynamical fission process is much more complex than the statical property of a nucleus in its ground state. For a long time, purely microscopic models were not able to attain the precision of the liquid-drop model in reproducing the macroscopic nuclear properties. Only very recently, the precision of fully microscopic and self-consistent models became comparable with the precision of macroscopic-microscopic models [11, 12]. While the powerful basic relations of the liquid-drop model follow directly from the theoretical assumption of a leptodermous system, the most reliable parameter values were determined by an adjustment to experimental masses and other nuclear properties. However, only microscopic models were able to relate the values of these model parameters to the properties of the nuclear force [13]. Remembering this analogy clarifies that GEF is not intended to compete with microscopic models, although GEF is presently better suited as far as use for applications is concerned. On the contrary, both approaches may be considered to be complementary for extracting physics. 


\section{B. Additional Ingredients}

For the calculation of fission observables it is not enough to master the dynamics of the fission process, starting from an excited compound nucleus and ending with the formation of two separated nuclei at scission. Also the initial reaction that induced the fission process, for example the capture of a neutron, must be described, eventually including pre-compound processes. Furthermore, the competition between particle emission, gamma emission and fission must be considered, because it determines the relative contributions of different fissioning systems with different excitation-energy distributions, if the initial excitation energy is high enough for multi-chance fission to occur. After scission, the fragments may be highly excited, and, thus, they emit a number of particles, mostly neutrons and gamma radiation. Also these processes are considered.

The GEF code aims to provide a complete description including the entrance channel and the de-excitation of the fragments. This is particularly important for the determination of the optimum parameters of the model, because all available observables should be included in the fit procedure. For this purpose, the algorithms should be very efficient in order to assure a short computing time. Therefore, whenever possible, approximations and analytical descriptions were preferred if they are precise enough not to alter the results beyond the inherent uncertainties of the model. More elaborate models that have been developed in many cases may later easily be implemented in the code.

\section{Understanding of the Fission Process}

The general character of the model makes it necessary to establish the systematics of the variation of the fission observables for different fissioning nuclei as a function of excitation energy and to interpret the origin of these features. It will be shown that the basic ideas of the model are astonishingly powerful. Therefore, the links between the observations and the ingredients of the model enable extraction of valuable information on the physics of the nuclear-fission process, much better than the inspection of isolated systems or the direct study of the measurements. An essential advantage provided by the model is the consistent description of all relevant degrees of freedom, due to the efficient computational technique of the code.

\section{Further Developments and Applications}

The GEF model is unique in the sense that it treats the complete fission process with explicit consideration of a large number of degrees of freedom in a coherent way on physics ground. Thus, it provides the links between the different processes and mechanisms and the fission observables. Even more, it preserves the correlations between the different degrees of freedom. It might be very useful for a better understanding of the fission process to carefully study any discrepancy between the model results and available experimental data. Moreover, the systematic trends and global features revealed or predicted by the model may stimulate dedicated experiments and calculations on specific problems with microscopic models in order to better understand certain aspects of the fission process.

While most of the available codes concentrate on specific observables, depending on the purpose, the GEF code provides a consistent description of all available experimental observables over a wide range, be they concerned with the properties of fragments, prompt neutrons, prompt gamma rays, or isomers. This is done without relying on the availability of specific experimental information, and with a single set of parameters. Moreover, GEF is a very fast code, meaning that the GEF code is suitable for large-scale model calculations, e.g. for a simulation of the influence of fission cycling on the astrophysical r-process [14].

In the application for nuclear data, the GEF code may replace purely parametric descriptions for many quantities and serve as a realistic, consistent and complete event generator for transport calculations with dedicated codes like MCNP [15] or FLUKA 16].

\section{E. Complementing, Estimating and Evaluating Nuclear Data}

Since the empirical data are an important input of the model, it is suggestive that GEF can be useful for the evaluation of nuclear data. First, the predictions of the model may directly be used for estimating some fission observables, if no experimental data are available. The code can also be useful in order to check whether certain experimental results are in line with or in contradiction with observed trends and systematics. This may lead to an enhanced or diminished confidence level of these data or eventually stimulate dedicated experiments for verification. A very useful application may be the exploitation of the code results for complementing missing data. For this purpose, a special algorithm has been developed that "fine-tunes" the calculated values in a way that they fit to the available experimental data. This algorithm is implemented in the computer code MATCH [17].

\section{BASIC IDEAS OF THE GEF MODEL}

This chapter gives a concise overview on the basic theoretical considerations of general validity that are employed in the GEF model. They define the specific physics content of the model and, moreover, they decisively determine the computational structure of the code and the range of applicability. Detailed informa- 
tion about the implementation in the GEF code is given separately in dedicated sections.

\section{A. Topological Properties of the Nuclear Potential in Multi-dimensional Space}

At first, the nucleus needs to leave the first minimum at its ground-state shape by passing the fission barrier, which in the actinides consists of two or more consecutive barriers with a minimum in between. The heights of these barriers have a strong impact on the fission-decay width. Thus, they are key quantities for spontaneous fission and for the relative weights of the different fission chances in multi-chance fission.

Myers and Swiatecki pointed out that the nuclear binding energy at the fission threshold, that is the binding energy at the highest one of the consecutive barriers between the ground-state shape and the scission configuration, is influenced only little by shell effects [18], meaning that the shell-correction energy $\delta U_{\text {sad }}$ is small. The basic idea is illustrated in Fig. 1, where the influence of the pairing condensation energy is neglected, because it cancels in first order. Thus, the height of the fission barrier $B_{f}$ is given to a good approximation by the difference of the macroscopic barrier $B_{f}^{m a c}$ and the shell-correction energy in the ground-state $\delta U_{g s}=E_{g s}^{e x p}-E_{g s}^{\text {mac }}$

$$
B_{f} \approx B_{f}^{m a c}-E_{g s}^{e x p}+E_{g s}^{m a c} .
$$

If the experimental ground-state energy $E_{g s}^{\exp }$ is not available, for example for very exotic nuclei, the shellcorrection energy in the ground-state $\delta U_{g s}$ in Eq. (1) may be estimated by a suitable macroscopic-microscopic model.

The condition for this topological property of a surface in multi-dimensional space is that the wavelength of the

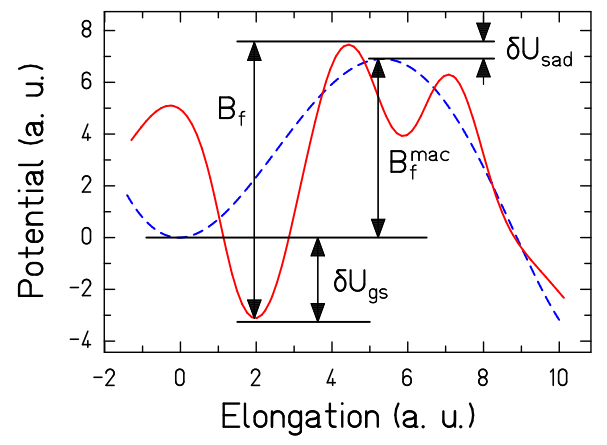

FIG. 1. (Color online) Schematic drawing of the potential energy on the fission path relative to the macroscopic groundstate energy $E_{g s}^{m a c}$ for a nucleus that is deformed in its ground state. Spherical shape corresponds to zero elongation. Blue dashed line: macroscopic potential. Red full line: full potential including the shell effect. fluctuations induced by the shell effects is smaller than the wavelength of the variations induced by the macroscopic potential. This behavior can be understood because a local modification of the potential by a bump or a dip, for example by shell effects, does not have a big effect on the height of the saddle: The fissioning nucleus will go around the bump, and it cannot profit from the depth of the dip, because the potential at its border has changed only little. A detailed investigation of the applicability of the topographic theorem was performed in Ref. [19]. The topographic theorem is exploited in the GEF model for deriving a precise semi-empirical systematics of fission barriers, see Sec. III A

\section{B. Statistical Filling of Quantum Oscillators}

Since tunneling occurs with a very low probability, as can be deduced from the long spontaneous-fission half lives, an excited nucleus has enough time to re-arrange its available energy before. The probability for the passage of the fission barrier increases considerably, if the nucleus concentrates enough of its energy on the relevant shape degrees of freedom for avoiding tunneling as much as the available energy allows. The remaining energy, however, can be randomly distributed between the different states above the barrier without any further restriction, such that the barrier is passed with maximum possible entropy on the average [20]. For this reason, the fissioning system has no memory on the configurations before the barrier, except the quantities that are preserved due to general conservation laws: total energy, angular momentum and parity. Thus, the starting point for calculating the properties of the fission fragments is the configuration above the outer fission barrier.

The evolution of the entropy plays a decisive role in the fission process. The concentration of a sufficiently large amount of energy into the elongation degree of freedom in order to overcome the fission barrier essentially without tunneling, if the total energy is sufficiently high, induces a reduction of the entropy. Moreover, the states at the barrier are populated according to their statistical weights. After passing the barrier, the entropy increases again due to dissipation. Therefore, we think that the approximation of treating fission as an isentropic process [21, 22] is not a generally valid assumption.

Beyond the outer barrier, one can define an optimum fission path, consisting of a sequence of configurations in deformation space with minimum potential energy for a certain elongation. (Strictly speaking, one should consider the dynamic fission path that includes the influence of dissipation and inertia.) Due to shell effects, structures emerge on the way from saddle to scission that produce additional fission valleys, defining competing fission paths. The fissioning system is unbound only with respect to one degree of freedom, the motion in direction of the fission path, and it is bound with respect to motion in any other direction in deformation space, for exam- 
ple in mass asymmetry or charge polarization at fixed elongation. The distribution of the collective coordinate is given by the occupation probability of the states of the quantum oscillators in the respective fission valleys. Some of these degrees of freedom that are confined by a restoring force towards the potential minimum are directly linked to fission observables, e.g. the mass asymmetry $\left(A_{1}-A_{2}\right) /\left(A_{1}+A_{2}\right)$ or the charge polarization $<Z_{1}>-Z_{U C D}$ [23] with $Z_{U C D}=A_{1} \cdot Z_{C N} / A_{C N} \cdot A_{C N}$, $Z_{C N}, A_{i}$ and $Z_{i}$ are mass and atomic number of the fissioning system and of one fragment, respectively.

The situation is schematically illustrated in Fig. 2 for the mass-asymmetry coordinate in two fission valleys that are well separated. The fission-fragment distribution in $Z$ and $A$ is given by the evolution of the respective collective variables, until the system reaches the scission configuration. It is defined by the number of occupied states in the different valleys. The value of the respective collective variable is the integral result of the forces, including the influence of dissipation and inertial forces, acting on the whole fission path.

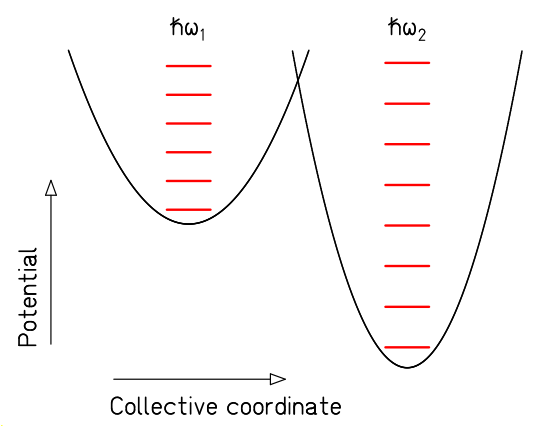

FIG. 2. (Color online) Schematic drawing of the potential energy as a function of a collective coordinate that is orthogonal to the fission direction at a fixed elongation. The two harmonic oscillator potentials with different depths and $\hbar \omega$ values represent the potential in two fission valleys for massasymmetric distortions that are related to different fission channels. The energies of the stationary states are indicated by the red horizontal lines. The overlapping of the two curves illustrates the possibility that the fission valleys are divided by a higher ridge that becomes perceptible when a continuous transition from one valley to the other is established in a deformation space with a sufficiently high dimension, see for example Ref. [24].

In the case of weak coupling and in thermal equilibrium with a heat bath of temperature $T$, the ratio of the yields $Y_{i}$ of the two fission channels corresponding to the population of the two harmonic quantum oscillators depicted in Fig. 2 is given by

$$
Y_{2} / Y_{1}=e^{-\Delta E / T} \cdot \frac{\hbar \omega_{1}}{\hbar \omega_{2}} \approx e^{-\Delta E / T} .
$$

$\Delta E$ is the potential-energy difference between the bottoms of the two quantum oscillators. This is the basic equation that is used in the GEF code to calculate the relative yields of the different fission channels at an elongation around the outer fission barrier. The relation is strongly dominated by the exponential term. For this reason, and because there are indications that the shape of the main asymmetric fission valley deviates from a Gaussian (see Sec. VIB), only the exponential term is used.

The distribution of the collective coordinate of a quantum oscillator, for example for asymmetric distortions in one fission channel, is a Gaussian function with a variance $\sigma^{2}$ that is given by the well known equation

$$
\sigma^{2}=\frac{\hbar \omega}{2 C} \operatorname{coth}\left(\frac{\hbar \omega}{2 T}\right)
$$

$C$ is the second derivative of the potential near its minimum in the direction of the collective coordinate considered.

\section{Early Localization of Nucleonic Wave Functions}

The observables from low-energy fission show strong manifestations of quantum-mechanical effects. These include (i) the contributions of the different fission channels to the fission-fragment mass and total-kinetic-energy distributions that are related to nuclear shell effects and (ii) the considerable enhancement of even- $Z$ fission fragments that are linked to pairing correlations. These quantummechanical features are responsible for a great part of the complexity of nuclear fission, and, thus, they considerably complicate the theoretical description of the fission process. The GEF model exploits a long-known general property of quantum mechanical wave functions in a strongly deformed potential in order to simplify this problem considerably, as described below.

When the two-center shell model became available, it was possible to study the single-particle structure in a dinuclear potential with a necked-in shape. Early investigations of Mosel and Schmitt 25] revealed that the singleparticle structure not far beyond the outer fission barrier already resembles very much the coherent superposition of the single-particle levels in the two separated fragments after fission, see Fig. 3. The authors explained this result by the general quantum-mechanical feature that wave functions in a slightly necked-in potential are already essentially localized in the two parts of the system. This feature is a direct consequence of the necking, independent of the specific shape parametrization. This finding immediately leads to the expectation that the shells on the fission path that are responsible for the complex structure of fission channels are essentially given by the fragment shells [26]. Potential-energy surfaces of fissioning systems calculated with the macroscopic-microscopic approach (e.g. Ref. [27]) support this assumption.

As a consequence, the shell effects on the fission path can be approximately considered as the sum of the shell effects in the proton- and neutron subsystems of the light and the heavy fission fragment. Thus, these shells do 


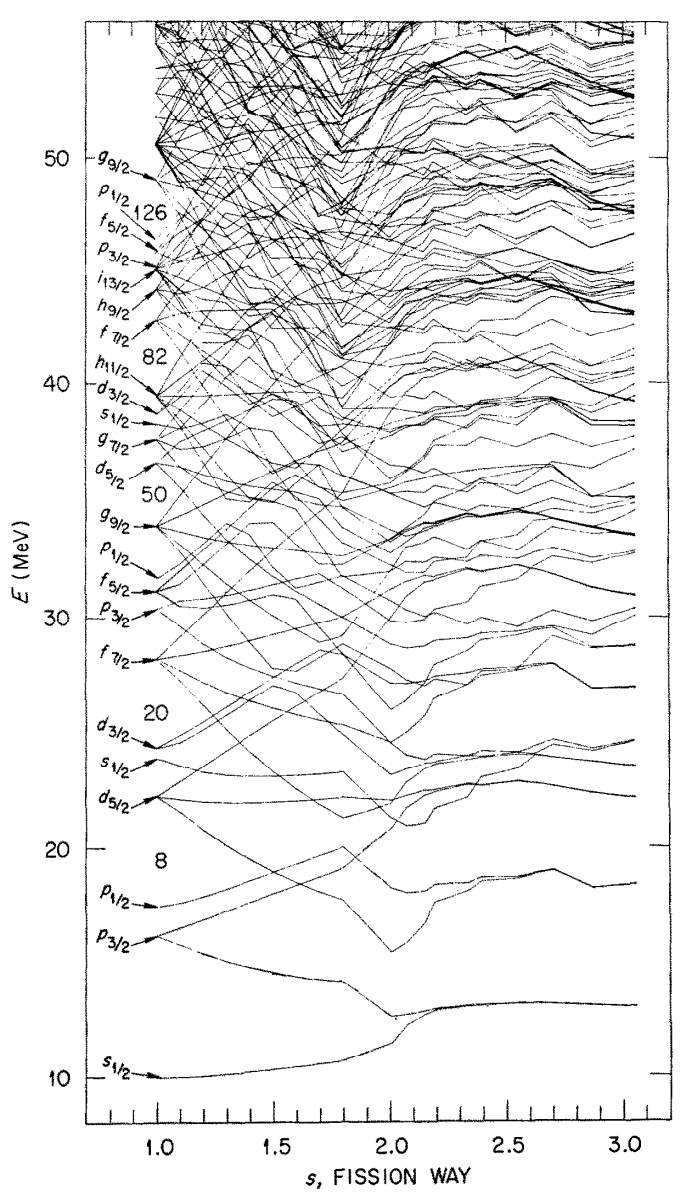

FIG. 3. Neutron shell-model states calculated with the twocenter shell model for the nucleus ${ }^{236} \mathrm{U}$. The coordinate $s$ characterizes the nuclear shape on the fission path. The figure covers the range from the spherical shape $(s=1)$ to a configuration with a neck radius of about $40 \%$ of the maximum extension of the system perpendicular to the symmetry axis $(s \approx 3.1)$. The outer saddle is located at $s=1.7$. The figure is taken from Ref. [25].

not primarily depend on the fissioning system but on the number of neutrons and protons in the two fission fragments. However, these shells may be substantially different from the shell effects of the fragments in their ground state, because the nascent fragments in the fissioning dinuclear system might be strongly deformed due to the interaction with the complementary fragment.

The potential energies at the bottom of the different fission valleys that determine the relative yields of the fission valleys according to Eq. (2) have 5 terms: the macroscopic potential and the shell energies of the proton and neutron subsystems of the two nascent fragments. In the GEF code, the last four terms are described by a common parametrization for all fissioning systems that is extracted from empirical data as described in Sec. III C The same is true for the potential curvatures $C$ that determine the widths of the distributions for specific fission channels according to Eq. (3). Also the temperature values that enter into Eqs. (2) and (3), including the variation with the fissioning system and its initial excitation energy as well as the different $\hbar \omega$ values, are empirically determined.

\section{Characteristic Times of Stochastic Processes}

Dynamical calculations revealed that, depending on the nature of the collective degree of freedom considered, dynamical effects induce a kind of memory on the fission trajectory due to the influence of dissipative and inertial forces [28]. The corresponding characteristic memory time determines, after which time a specific coordinate value is forgotten and how long it takes for this coordinate to adjust to modified conditions. This means that the distribution of a specific observable is essentially determined by the properties of the system, for example the potential-energy surface, at an earlier stage. According to experimental observations [29] and theoretical studies [30], the mass distribution is essentially determined already well before reaching scission. The memory of the charge-polarization degree of freedom has been found to be much shorter [31, 32].

As a practical consequence, the measured distribution of a specific fission observable essentially maps a kind of effective potential the system was exposed to by the characteristic memory time before reaching scission. In other words, the effective potential that is extracted from the measured distribution of a fission observable implicitly includes the influence of dynamical effects. It cannot be interpreted as the potential energy at a specific elongation, e. g. at scission.

Stochastic calculations [6] suggest that the fluctuations in mass asymmetry continue to grow after the decision for a specific fission channel is already made. In the picture of coupled quantum oscillators, this suggests that the coupling between the different oscillators vanishes rather early, while the collective temperature still increases on the way to scission. Thus, the two temperature values in Eqs. (2) and (3) are separately determined by an adjustment to the characteristics of the yields and the widths of the fission channels, respectively.

\section{E. Entropy-driven Energy Sorting}

The transformation of energy between potential energy, intrinsic and collective excitations as well as kinetic energy is another very important aspect of the nuclearfission process. It determines the partition of the fission Q value (plus eventually the initial excitation energy of the fissioning system) between kinetic and excitation energy of the final fragments. Moreover, the division of the total excitation energy between the fragments is of considerable interest, because it induces a shift of the isotopic distributions from the primary fragments by neutron evap- 
oration towards less neutron-rich isotopes. It has also been noticed that it has a practical consequence for the interpretation of other experimental data: The shape of the mass-dependent prompt-neutron yields strongly affects the mass values of the fission products deduced from kinematical double-energy measurements 33].

The GEF model takes advantage of the general laws of statistical mechanics, which govern the energetics of any object, independently of its size [34]. In particular, statistical mechanics requires that the available energy tends to be distributed among the accessible degrees of freedom in equal share during the dynamical evolution of the system. This general law provides an estimation of the evolution of the intrinsic excitation energies and the population of the available states in the nascent fragments during the fission process with little computational expense. In the super-fluid energy domain, where the level density has been found to be well approximated by a constant-temperature formula, this leads to the phenomenon of entropy-driven energy sorting [35].

\section{F. Exploiting Empirical Knowledge}

Many of the ideas outlined in the previous sections establish a link between measured observables and specific properties of the fissioning system. It is an essential part of the GEF concept that this empirical information is exploited to assure that the quantitative results of the model are in best possible agreement with the available experimental data. For this purpose, the ingredients of the model and its parameter values are adjusted in a global fit procedure that minimizes the deviations from a large set of experimental data of different kind. Note that the GEF model, in contrast to current empirical models, is not a direct parametrization of the observables. Instead, as was mentioned above, it describes the fission quantities, making use of several approximations based on general properties of microscopic systems. The quality with which the GEF code is able to reproduce a large body of data with a moderate number of parameters will give an indication about the validity of these approximations.

\section{G. Generality}

From a theoretical model, one requires that the evolution of the fissioning system is fully described, considering all degrees of freedom, their dependencies and their correlations. Current microscopic models, either classical or quantum-mechanical ones, do not meet this requirement, because they only consider a restricted number of degrees of freedom for the parametrization of the shape and other properties of the system, or for the external constraint, respectively. Statistical models applied at the saddle or at the scission point are not suited neither, because they neglect the dynamical aspects of the fission process.
The GEF model is a compromise that does not eliminate any of the complex features of the fission process by far-reaching approximations or restrictions from the beginning on. Instead, it makes use of a number of generally valid physics laws and characteristics that allow reducing the computing expenses to an affordable level.

\section{H. Correlations and Dependencies}

Nuclear fission provides a large number of observables. The correlations between different kind of observables represent valuable information on the fission process. Therefore, the GEF code is designed as a Monte-Carlo code that follows all quantities of the fissioning systems with their correlations and dependencies. Finally, all observables can be listed event by event, which allows the investigation of all kind of correlations. Moreover, complex experimental filters can easily be implemented as was done for the planning of the SOFIA experiment [36] by using GEF as an event generator for the CONFID code 37] that calculates the trajectories of the fission fragments in an experimental set-up that includes a dipole magnet.

\section{Range of Validity}

According to the concept of the GEF model, the range of validity is not strictly defined. Technically, the code runs for any heavy nucleus. However, the results of the model are more reliable for nuclei which are not too far from the region where experimental data exist. It is recommended not to use the code outside the range depicted in Fig. 4 on the chart of the nuclides.

Ternary fission is not covered.

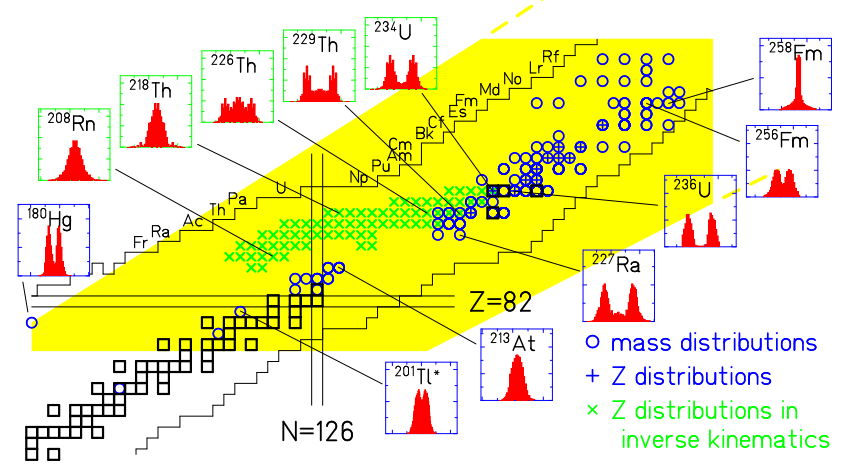

FIG. 4. (Color online) Validity range of the GEF model. The validity range of the GEF model is marked in yellow. For a detailed description of the figure see Fig. 9 


\section{MODELING OF THE FISSION PROCESS}

This section describes the modeling of the fission process in the GEF code, starting from a specific compound nucleus that is characterized by its excitation energy and angular momentum and ending with the emission of prompt neutrons and gamma radiation from the fully accelerated fragments. Entrance-channel effects and the treatment of multi-chance fission are considered in separate sections. Emission of delayed neutrons is considered as well in the context of applications.

Some specific formulations and parameter values that will presumably be subject to modifications in the further development of the GEF code, because they are adjusted to the body of experimental data which is continuously being extended and improved, are listed separately in a dedicated section.

\section{A. Systematics of Fission Barriers}

One of the most critical input parameters of the GEF model is the height of the fission barrier. That is the energy a nucleus has to invest in order to proceed to fission without tunneling. Since experimental fission barriers are available for a rather restricted number of nuclei, only, a model description is needed in order to meet the requirement of the GEF model for generality.

An elaborate analysis 38] of available experimental data revealed that different theoretical models differ appreciably in their predictions for the average trend of the fission-barrier height along isotopic chains. Also selfconsistent models deviate drastically from each other.

During the last years, the efforts for developing improved models for the calculation of fission barriers were intensified, using the macroscopic-microscopic approach [27, 39 44], the density-functional theory 22, 45, 46] and varieties of Hartree-Fock methods [47 50]. Still, the results from the different models, in particular in regions, where no experimental data exist, differ appreciably. Since the fission-barrier height is the difference of the mass at the saddle-point which defines the fission barrier and the ground-state mass, it is obvious that the fissionbarrier values from these models cannot be more precise than the values of the ground-state masses, which show typical root-mean square deviations of at least several 100 $\mathrm{keV}$ from the experimental values. This results from the assumption that the deviations in reproducing the fission barriers are partly caused by deficiencies of the models for estimating the microscopic contributions to the binding energies in the ground state and at saddle, and that these two contributions are essentially independent from each other, regarding the complex level crossings in the Nilsson diagram [52] and the fact that the topographic theorem is only valid at the barrier.

An alternative approach that avoids this problem was used in Ref. [51], by estimating the fission barrier as the sum of the macroscopic fission barrier and the shell- correction energy in the ground-state, making use of the topographic theorem [18, 19]. In the GEF model, we follow this idea. However, we explicitly consider the pairing condensation energies in the ground state and at the barrier, because they are systematically different. For the macroscopic part of the fission barrier, we chose the Thomas-Fermi barriers of Myers and Swiatecki [53] and combined them with the Thomas-Fermi masses of the same authors [18] for determining the contributions of shells and pairing to the ground-state binding energy, because these models were found [38] to follow best the isotopic trends of the experimental masses and fission barriers.

In detail, the macroscopic fission-barrier height for the nucleus with mass number $A$ and atomic number $Z$ is calculated with the following relations, adapted from Ref. [53]:

$$
N=A-Z
$$

$$
\begin{aligned}
& I=(N-Z) / A \\
& \kappa=1.9+(Z-80) / 75 \\
& S=A^{2 / 3}\left(1-\kappa I^{2}\right) \\
& X=\frac{Z^{2}}{A\left(1-\kappa I^{2}\right)}
\end{aligned}
$$

For $30 \leq X<X_{1}$ :

$$
F=0.595553-0.124136\left(X-X_{1}\right)
$$

For $X \geq X_{1}$ and $X \leq X_{0}$ :

$$
F=0.000199749\left(X_{0}-X\right)^{3}
$$

with $X_{0}=48.5428$ and $X_{1}=34.15$. Finally, the ThomasFermi macroscopic fission barrier is given by:

$$
B_{f}^{T F} / \mathrm{MeV}=F \cdot S .
$$

The higher one of the inner $\left(B_{A}\right)$ and the outer $\left(B_{B}\right)$ fission barrier $\left(B_{f}=\max \left(B_{A}, B_{B}\right)\right)$ is given by the sum of the macroscopic fission barrier and the negative value of the microscopic contribution to the ground-state mass $\delta E_{g s}^{m i c}$ plus the microscopic contribution $\delta E_{f}^{m i c}$ to the binding energy at the respective barrier by:

$$
B_{f}=B_{f}^{T F}-\delta E_{g s}^{m i c}+\delta E_{f}^{m i c} .
$$

The microscopic contribution to the ground-state mass is the difference of the actual ground-state mass and the 
macroscopic mass obtained with the Thomas-Fermi approach $\delta E_{g s}^{m i c}=m_{g s}-m_{T F}$. It represents the structural variation of the ground-state mass due to shell correction $\delta U_{g s}$ and pairing condensation energy $\delta P_{g s}$. If available, the values of $m_{g s}$ are taken from the 2012 mass evaluation. Otherwise, the calculated shell-correction energies from Ref. [54] were used. The topographic theorem claims that the shell effect at the barrier can be neglected. Therefore, only the contribution from pairing must be considered: $\delta E_{f}^{m i c}=\delta P_{f}$. Best agreement with the data is obtained by including the even-odd staggering of the binding energy at the barrier with $\delta P_{f}^{\prime}=-n \cdot 14$ $\mathrm{MeV} / \sqrt{A}, n=0,1,2$ for odd-odd, odd-mass and eveneven nuclei, respectively. Note that the quantity $\delta P_{f}^{\prime}$ represents only part of the pairing condensation energy $\delta P_{f}$. The remaining, slowly varying, part is hidden in the macroscopic barriers of Ref. [53]. Thus, the final equation is

$$
B_{f}=B_{f}^{T F}-\left(m_{g s}-m_{T F}\right)+\delta P_{f}^{\prime} .
$$

The available measured fission barriers were used to deduce the following empirical function, which describes the difference between the inner and the outer barrier height:

$$
\begin{array}{r}
\left(B_{A}-B_{B}\right) / \mathrm{MeV}=5.40101- \\
0.00666175 \cdot Z^{3} / A+1.52531 \cdot 10^{-6} \cdot\left(Z^{3} / A\right)^{2} .
\end{array}
$$

The result of this procedure was not yet fully satisfactory, because the barriers around thorium were somewhat overestimated. This discrepancy decreases for lighter and for heavier elements. Fig. 5 illustrates a possible reason for this deviation in a schematic way: The lower part shows the macroscopic potential, essentially given by the

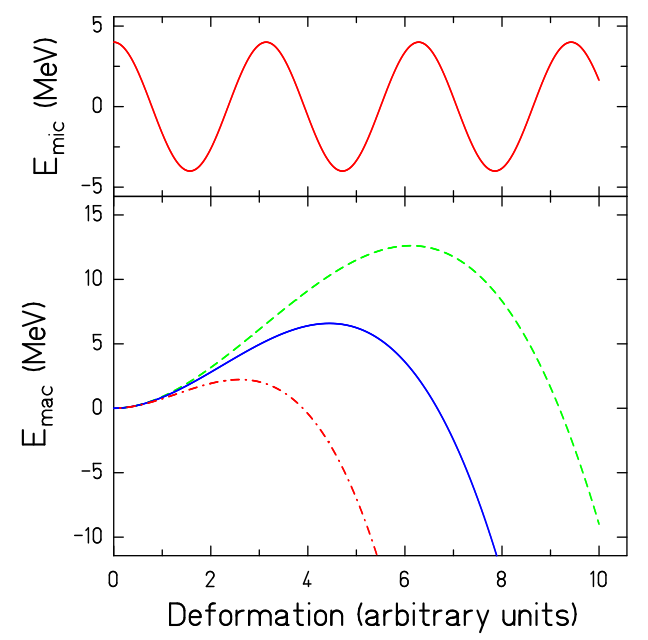

FIG. 5. (Color online) Schematic drawing of the microscopic and the macroscopic potential in fission direction. See text for details. asymmetry-dependent surface energy and Coulomb interaction potential, for a lighter (dashed line), for a mediumheavy (full line) and for a heavier (dot-dashed line) nucleus. The upper part shows the schematic variation of the shell correction as a function of deformation, which is assumed to be the same for all nuclei in the region of the heavy nuclei concerned. The full potential can be assumed as the sum of the macroscopic and the microscopic potential. The first minimum of the nuclear ground state is deformed in the actinides considered. The full line in the lower part corresponds to the situation around thorium: The inner and the outer barriers have about the same height. This situation is realized when the second minimum is localized near the maximum of the macroscopic potential. In this case, the inner and the outer barriers are localized at deformations, where the macroscopic potential is far from its maximum value. For lighter nuclei, the maximum of the macroscopic potential moves to larger deformations, closer to the outer barrier, which becomes the higher one. For heavier nuclei, the maximum of the macroscopic potential moves to smaller deformations, closer to the inner barrier, which becomes the higher one. This consideration makes it understandable that the barriers of nuclei around thorium deviate systematically from the smooth trend expected from the topographic theorem: They are systematically smaller. This deviation was parametrized by the following correction term:

For $86.5<Z<90$

$$
\Delta B_{f} / \mathrm{MeV}=-0.15(Z-86.5),
$$

for $90 \leq Z<93$

$$
\Delta B_{f} / \mathrm{MeV}=-0.15(Z-86.5)+0.35(Z-90),
$$

for $93<Z<95$

$$
\begin{aligned}
\Delta B_{f} / \mathrm{MeV}=-0.15(Z-86.5) & +0.35(Z-90) \\
& +0.15(Z-93),
\end{aligned}
$$

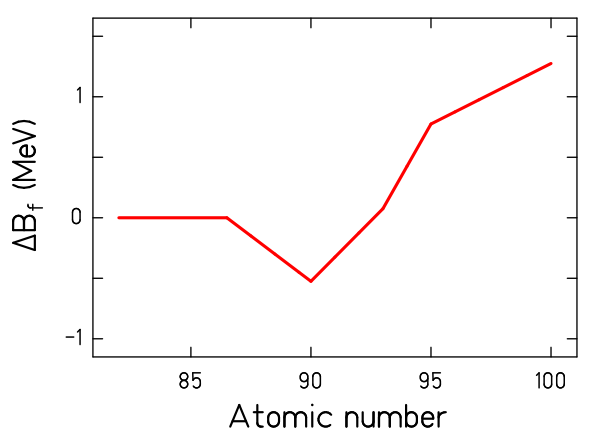

FIG. 6. (Color online) Empirical correction applied to the fission-barrier height obtained with the topographic theorem as a function of the atomic number of the fissioning nucleus. 
and for $Z \geq 95$

$$
\begin{aligned}
\Delta B_{f} / \mathrm{MeV}=- & 0.15(Z-86.5)+0.35(Z-90) \\
& +0.15(Z-93)-0.25(Z-95) .
\end{aligned}
$$

The resulting function is depicted in Fig. 6. In addition to the dip around $Z=90$, which can be considered as a refinement of the topographic theorem, the barrier heights had to be further increased for the heavier elements in order to better reproduce the measured values. This latter effect, which is the only violation of the topographic theorem in our description, may be caused by shell effects at the barrier or by a shortcoming of the Thomas-Fermi barriers for the heaviest elements.

The fission-barrier values of several heavy nuclei that are used in the GEF code are listed in Tables I and II In addition, they are compared in Figs. 7 and 8 with different empirical and theoretical values. The values of Goriely et al. [50], which result from a normalization of theoretical barriers obtained with a self-consistent model to measured neutron-induced fission cross sections, are quite close to the empirical data. In contrast, the values of Möller et al. 27, 55], which directly result from a macroscopic-microscopic model, deviate strongly in their absolute values and in the isotopic trends. Obviously, the description used in the GEF code agrees rather well with the empirical data. In particular, the good agreement with the isotopic trend in each of the isotopic sequences for the inner and for the outer barrier suggests that this description can be extrapolated further away from the beta-stable region without any new assumptions.

TABLE I. Fission barriers used in GEF, part 1.

\begin{tabular}{c|c|c|c|c|c}
\hline \hline $\mathrm{N}$ & $\mathrm{Th}$ & $\mathrm{Pa}$ & $\mathrm{U}$ & $\mathrm{Np}$ & $\mathrm{Pu}$ \\
\hline 134 & $5.75 / 5.87$ & $4.99 / 4.79$ & $4.12 / 3.57$ & $3.41 / 2.46$ & $3.20 / 1.83$ \\
135 & $5.84 / 6.01$ & $5.53 / 5.39$ & $4.45 / 3.95$ & $4.18 / 3.29$ & $3.48 / 2.18$ \\
136 & $5.64 / 5.85$ & $5.25 / 5.15$ & $4.54 / 4.10$ & $4.07 / 3.25$ & $3.81 / 2.57$ \\
137 & $5.70 / 5.96$ & $5.64 / 5.60$ & $4.84 / 4.46$ & $4.67 / 3.91$ & $4.30 / 3.13$ \\
138 & $5.67 / 5.98$ & $5.44 / 5.45$ & $5.00 / 4.67$ & $4.64 / 3.94$ & $4.37 / 3.27$ \\
139 & $5.78 / 6.13$ & $5.88 / 5.93$ & $5.35 / 5.07$ & $5.22 / 4.58$ & $4.79 / 3.75$ \\
140 & $5.70 / 6.09$ & $5.91 / 6.43$ & $5.37 / 5.15$ & $5.24 / 4.66$ & $4.89 / 3.92$ \\
141 & $5.91 / 6.34$ & $6.03 / 6.18$ & $5.85 / 5.67$ & $5.81 / 5.29$ & $5.43 / 4.52$ \\
142 & $5.72 / 6.19$ & $5.60 / 5.70$ & $5.69 / 5.56$ & $5.60 / 5.13$ & $5.37 / 4.53$ \\
143 & $5.84 / 6.35$ & $6.03 / 6.18$ & $5.95 / 5.87$ & $6.08 / 5.66$ & $5.80 / 5.01$ \\
144 & $5.65 / 6.19$ & $5.72 / 5.91$ & $5.76 / 5.73$ & $5.70 / 5.34$ & $5.65 / 4.92$ \\
145 & $5.87 / 6.46$ & $6.06 / 6.30$ & $6.04 / 6.06$ & $6.19 / 5.88$ & $6.08 / 5.41$ \\
146 & $5.56 / 6.18$ & $5.48 / 5.84$ & $5.64 / 5.71$ & $5.68 / 5.43$ & $5.70 / 5.09$ \\
147 & $5.71 / 6.37$ & $5.77 / 6.17$ & $5.82 / 5.94$ & $5.97 / 5.76$ & $5.97 / 5.41$ \\
148 & $5.19 / 5.89$ & $5.48 / 5.84$ & $5.44 / 5.59$ & $5.63 / 5.47$ & $5.61 / 5.10$ \\
149 & & $5.77 / 6.17$ & $5.63 / 5.83$ & $5.98 / 5.87$ & $5.89 / 5.44$ \\
150 & & $5.28 / 5.72$ & $5.19 / 5.43$ & $5.36 / 5.30$ & $5.49 / 5.09$ \\
151 & & & $5.29 / 5.57$ & $5.77 / 5.75$ & $5.65 / 5.31$ \\
152 & & & $5.63 / 5.95$ & $5.04 / 5.07$ & $5.30 / 5.01$ \\
153 & & & & $5.96 / 6.03$ & $5.34 / 5.10$ \\
154 & & & & &
\end{tabular}

Note: Height of first and second barrier used in the GEF code in $\mathrm{MeV}$.
The even-odd staggering of the fission-barrier height is well reproduced by the model, assuming a pairing-gap parameter $\Delta=14 \mathrm{MeV} / \sqrt{A}$, compared to an average value of $\Delta=12 \mathrm{MeV} / \sqrt{A}$ in the nuclear ground state. This may eventually be evidence for the deformation dependence of the pairing strength [56]. But a stronger pairing at the barrier is also expected by the systematically higher single-particle level density at the barrier compared to the ground state due to topological reasons: while the barrier is practically not lowered by shell effects compared to the macroscopic barrier, the nuclear ground state is almost generally more bound than the macroscopic ground state, because it is the state with the absolute lowest energy in deformation space. Generally, more binding by shell effects is related to a lower single-particle level density at the Fermi level. This kind of even-odd staggering is also present in the theoretical values [27] and [50], although the amplitude is not large enough.

The rms deviations between the different sets of fission barriers shown in Figs. 7 and 8 are listed in Table III. There is a remarkably large deviation between the empirical barriers from Bjornholm and Lynn [57] that are extracted from measured fission probabilities and the recommended values of RIPL 3 [58]. The best agreement exists between the GEF parametrization and the empiri-

TABLE II. Fission barriers used in GEF, part 2.

\begin{tabular}{c|c|c|c|c|c}
\hline \hline $\mathrm{N}$ & $\mathrm{Am}$ & $\mathrm{Cm}$ & $\mathrm{Bk}$ & $\mathrm{Cf}$ & $\mathrm{Es}$ \\
\hline 137 & $4.35 / 2.73$ & $3.77 / 1.67$ & & & \\
138 & $4.28 / 2.74$ & $4.05 / 2.03$ & $3.64 / 1.11$ & & \\
139 & $4.98 / 3.51$ & $4.45 / 2.51$ & $4.27 / 1.82$ & $3.89 / 0.89$ & \\
140 & $5.02 / 3.62$ & $4.60 / 2.74$ & $4.41 / 2.04$ & $4.12 / 1.21$ & $3.95 / 0.46$ \\
141 & $5.75 / 4.42$ & $5.16 / 3.37$ & $5.11 / 2.82$ & $4.68 / 1.85$ & $4.61 / 1.21$ \\
142 & $5.67 / 4.41$ & $5.21 / 3.49$ & $5.12 / 2.92$ & $4.77 / 2.04$ & $4.59 / 1.28$ \\
143 & $6.23 / 5.03$ & $5.70 / 4.06$ & $5.69 / 3.57$ & $5.22 / 2.58$ & $5.22 / 2.01$ \\
144 & $5.97 / 4.84$ & $5.64 / 4.07$ & $5.63 / 3.58$ & $5.27 / 2.70$ & $5.18 / 2.06$ \\
145 & $6.50 / 5.43$ & $6.10 / 4.60$ & $6.16 / 4.19$ & $5.70 / 3.22$ & $5.73 / 2.70$ \\
146 & $6.05 / 5.04$ & $5.77 / 4.34$ & $5.77 / 3.88$ & $5.57 / 3.17$ & $5.55 / 2.69$ \\
147 & $6.39 / 5.45$ & $6.08 / 4.71$ & $6.23 / 4.41$ & $5.94 / 3.62$ & $6.08 / 3.23$ \\
148 & $5.90 / 5.01$ & $5.82 / 4.52$ & $5.95 / 4.19$ & $5.85 / 3.61$ & $5.83 / 3.06$ \\
149 & $6.30 / 5.48$ & $6.19 / 4.95$ & $6.51 / 4.83$ & $6.37 / 4.21$ & $6.43 / 3.74$ \\
150 & $5.73 / 4.96$ & $5.82 / 4.65$ & $6.05 / 4.44$ & $6.09 / 4.00$ & $6.22 / 3.62$ \\
151 & $5.99 / 5.28$ & $6.07 / 4.96$ & $6.41 / 4.87$ & $6.36 / 4.35$ & $6.73 / 4.20$ \\
152 & $5.50 / 4.84$ & $5.69 / 4.64$ & $5.94 / 4.46$ & $6.02 / 4.08$ & $6.35 / 3.90$ \\
153 & $5.66 / 5.06$ & $5.71 / 4.72$ & $6.02 / 4.61$ & $6.05 / 4.18$ & $6.35 / 3.98$ \\
154 & $5.01 / 4.47$ & $5.18 / 4.25$ & $5.26 / 3.91$ & $5.48 / 3.68$ & $5.78 / 3.49$ \\
155 & $5.34 / 4.84$ & $5.11 / 4.24$ & $5.35 / 4.07$ & $5.43 / 3.70$ & $5.82 / 3.60$ \\
156 & & $4.64 / 3.83$ & $4.70 / 3.48$ & $4.95 / 3.29$ & $5.10 / 2.96$ \\
157 & & & $4.85 / 3.69$ & $4.91 / 3.31$ & $5.23 / 3.17$ \\
158 & & & $4.51 / 3.41$ & $4.46 / 2.93$ & $4.62 / 2.62$ \\
159 & & & & $4.03 / 3.17$ & $4.76 / 2.84$ \\
160 & & & & $4.41 / 3.01$ & $4.79 / 2.94$ \\
161 & & & & & $5.17 / 3.39$ \\
162 & & & & & \\
\hline \hline
\end{tabular}

Note: Height of first and second barrier used in the GEF code in $\mathrm{MeV}$. (Continuation of Table I.) 

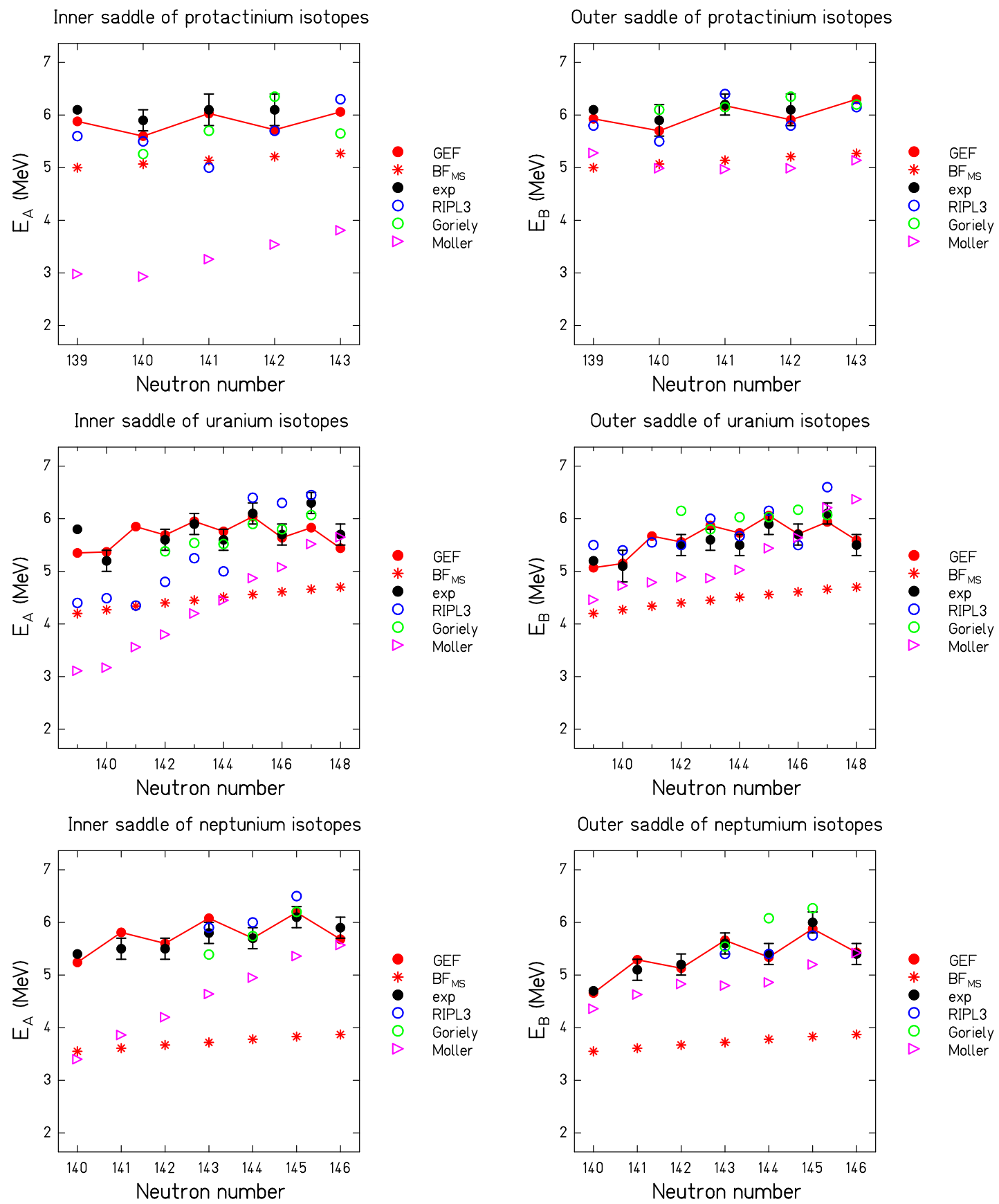

FIG. 7. (Color online) Overview of fission barriers, part 1. Height of the inner and the outer fission barrier above the nuclear ground state for isotopes of protactinium, uranium and neptunium. The description used in the GEF model is compared with the empirical barriers determined in Ref. [57] (marked by "exp"), with the recommended barriers given in RIPL3 [58], with the normalized self-consistent barriers [50] given in RIPL3 (marked by "Goriely"), and with the macroscopic-microscopic barriers of Möller et al. 27]. In addition, the macroscopic fission barriers $\left(B F_{M S}\right)$ from Ref. [53] are shown. Note that the symbols of the barriers from GEF are hardly visible, because they are covered in several cases by the experimental points.

cal values determined by Bjornholm and Lynn [57]. The rms deviation of $0.2 \mathrm{MeV}$ does not exceed the estimated uncertainties of the experimental values [57] and is appreciably smaller than the rms deviations with which the best atomic mass models reproduce the experimental values 60]. Also, the normalised self-consistent barriers of Goriely et al. [50] agree better with the empirical values of Bjornholm and Lynn than with the recommended values 

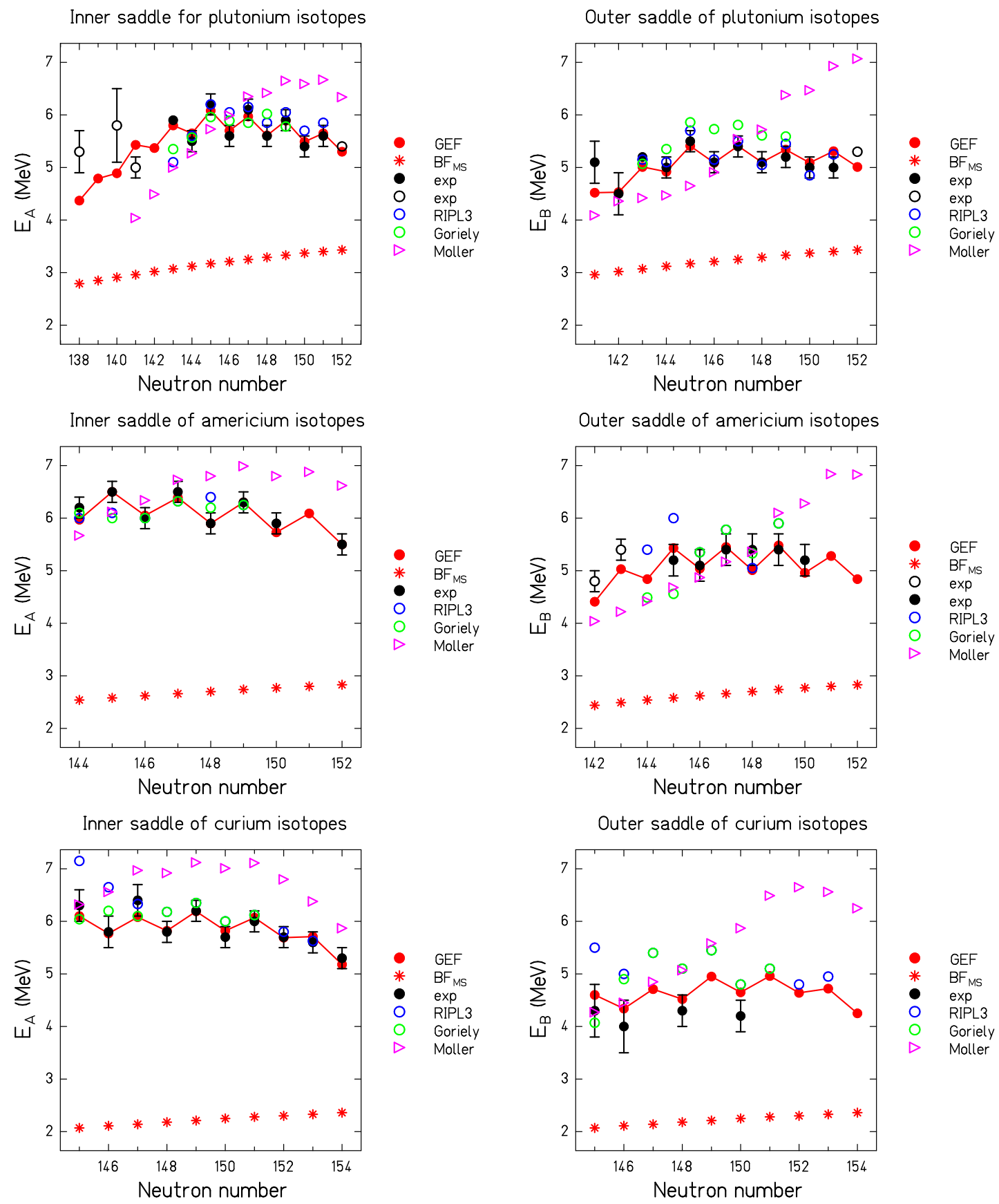

FIG. 8. (Color online) Overview of fission barriers, part 2. Height of the inner and the outer fission barrier above the nuclear ground state for isotopes of plutonium, americium and curium. The description used in the GEF model is compared with the empirical barriers (marked by "exp") determined in Ref. [57] (full symbols) and other papers cited in Ref. 59] (open symbols), with the recommended barriers given in RIPL3 [58], with the normalized self-consistent barriers [50] given in RIPL3 (marked by "Goriely"), and with the macroscopic-microscopic barriers of Möller et al. 27]. In addition, the macroscopic fission barriers $\left(B F_{M S}\right)$ from Ref. [53] are shown. Note that the symbols of the barriers from GEF are hardly visible, because they are covered in several cases by the experimental points.

of RIPL $3[\underline{58}]$. From the figures, one can deduce that the data from Ref. [57] and the GEF parametrization, which is deduced from the topographic theorem, agree best in the isotopic trend, while the theoretical values of Ref. 50. and the RIPL 3 values show increasingly discrepant local deviations. The theoretical values of Möller et al. deviate 
most strongly from any other set. Considering that the only adjustment of the proposed description is the application of the simple and well justified global $Z$-dependent function shown in Fig. 6, experiment and calculation are fully independent in their structural features and in their global dependency on neutron excess. Most remarkable is the great similarity in the structures along isotopic chains that are not modified by the corrections of Fig. 6, because these only depend on the atomic number of the fissioning system. The good agreement evidences that the barriers obtained with the present approach represent the empirical values or Ref. [57] better than the two theoretical models considered or the RIPL-3 recommended values.

\section{B. Nuclear Level Densities}

Nuclear level densities are another important ingredient of any nuclear model. There exist several descriptions that differ substantially, in particular in their low-energy characteristics. A recent analysis revealed that many of these descriptions are not consistent with our present understanding of nuclear properties 63. The result can be summarized as follows:

1. The even-odd staggering of the nuclear binding energies proves that pairing correlations are present in essentially all nuclei at low excitation energies. Therefore, any kind of level-density formula based on the so-called Fermi-gas level-density, which is derived under the independent-particle assumption, is not valid in the low-energy regime.

2. Since pairing correlations are only stable, if they enhance the nuclear binding, the binding energies of all nuclei are enhanced with respect to the value obtained in the independent-particle picture. Therefore, the level-density description for energies above the regime of pairing correlations, for example by a Fermi-gas formula, must be constructed with an energy scale that starts at an energy above the ground state for all nuclei. States at an excitation energy below this energy-shift parameter exist only due the

TABLE III. Rms deviation between different sets of fission barriers.

\begin{tabular}{c|c|c|c|c|c}
\hline \hline & exp & RIPL 3 & GEF & Goriely & Möller \\
\hline exp & - & 0.43 & 0.20 & 0.37 & 1.1 \\
RIPL 3 & 0.43 & - & 0.46 & 0.46 & 1.0 \\
GEF & 0.20 & 0.46 & - & 0.38 & 1.1 \\
Goriely & 0.37 & 0.46 & 0.38 & - & 1.0 \\
Möller & 1.1 & 1.0 & 1.1 & 1.0 & - \\
\hline \hline
\end{tabular}

Note: The table lists the rms deviations in $\mathrm{MeV}$ between the different sets of fission barriers shown in Figs. 7 and 8 . References are given in the figure captions. The typical uncertainty of the experimental values is 0.2 to $0.3 \mathrm{MeV}$. gain of binding that result from the pairing correlations.

3. From an almost constant-temperature behavior of measured level densities, high heat capacities are deduced for nuclei up to excitation energies in the order of $10 \mathrm{MeV}$. Wiggles in the heat capacity prove that the high heat capacity is caused by the consecutive creation of internal degrees of freedom by pair breaking, such that the energy per degree of freedom stays approximately constant as a function of excitation energy in the regime of pairing correlations.

In the GEF model, a simple and transparent analytical description is used that meets the above-mentioned requirements. The nuclear level density was modeled by the slightly simplified constant-temperature description of von Egidy and Bucurescu [64] at low energies

$$
\rho_{C T}=\frac{1}{T} e^{\left(E-E_{0}\right) / T}
$$

with

$$
E_{0}=-n \cdot \Delta
$$

( $n=0,1,2$ for even-even, odd- $A$, and odd-odd nuclei, respectively, and $\Delta=12 \mathrm{MeV} / \sqrt{A}$ ). The temperature parameter $T$ depends on the mass $A$ of the nucleus and the shell-correction energy $\delta U$

$$
T / \mathrm{MeV}=\frac{A^{-2 / 3}}{0.0597+0.00198 \cdot \delta U / \mathrm{MeV}} .
$$

In the ground-state shape this is the ground-state shell effect. The same formula is applied in different configurations, e.g. at the fission barrier, where the shell effect is basically different from the ground-state shell effect, however with a larger pairing-gap parameter $\Delta=14$ $\mathrm{MeV} / \sqrt{A}$.

The level density was smoothly joined at higher energies with the modified Fermi-gas description of Ignatyuk et al. 65, 66] for the total nuclear level density

$$
\rho_{F G}=C_{F G} \frac{e^{2 \sqrt{\tilde{a} U}}}{\tilde{a}^{1 / 4} U^{5 / 4}}
$$

with $U=E+E_{\text {cond }}+\delta U(1-\exp (-\gamma E)), \gamma=$ $0.055 / \mathrm{MeV}$ and the asymptotic level-density parameter $\tilde{a}=\left(0.078 A+0.115 A^{2 / 3}\right) / \mathrm{MeV}$. The shift parameter is $E_{\text {cond }}=-2 \mathrm{MeV}-n \Delta, \Delta=12 \mathrm{MeV} / \sqrt{A}(\Delta=14$ $\mathrm{MeV} / \sqrt{A}$ at the barrier) with $n=0,1,2$, for odd-odd, odd- $A$ and even-even nuclei, respectively, as proposed in Ref. 63 ]. $\delta U$ is the shell correction. The matching energy, which is equal to the critical pairing energy, is determined from the matching condition (continuous level-density values and derivatives of the constant-temperature and the Fermi-gas parts) This condition fixes the factor $C_{F G}$ in Eq.(22). This factor includes a collective-enhancement 
factor of about 100, which is close to the theoretically expected value [67].

The proposed modeling of the nuclear level density avoids two problems of the standard Gilbert-Cameron composite level-density description [68], which does not have room for a collective-enhancement factor and which imposes a loss of nuclear binding by pairing correlations in many nuclei, see Ref. 63]. This is in conflict with the fact that pairing correlations are only stable, if they increase the nuclear binding. The constant-temperature range reaches to about 8 to $10 \mathrm{MeV}$, which is appreciably higher than the matching energy in the standard GilbertCameron composite level-density description. This is an important feature for the energy-sorting process described below and for a weak sensitivity of the shape of the prompt-neutron spectra to the total excitation energy TXE of the fission fragments.

\section{Empirical Fragment Shells}

Quantitative predictions of the fission-fragment yields with the GEF code rely on the properties of the fragment shells that define the quantum oscillators in mass asymmetry and charge polarization on the fission path. The present section describes the underlying ideas and the applied procedure, how these are deduced from the measured fission-fragment distributions.

Fig. 9 gives an overview on the measured mass and nuclear-charge distributions of fission products from lowenergy fission. Fission of target nuclei in the actinide region, mostly induced by neutrons, shows predominantly asymmetric mass splits. A transition to symmetric mass splits is seen around mass 258 in spontaneous fission of fusion residues. Electromagnetic-induced fission of relativistic secondary beams covers the transition from asymmetric to symmetric fission around mass 226 [69]. A pronounced fine structure close to symmetry appears in ${ }^{201} \mathrm{Tl}$ [70] and in ${ }^{180} \mathrm{Hg}$ [71]. It is difficult to observe low-energy fission in this mass range. Thus, ${ }^{201} \mathrm{Tl}$ could only be measured down to $7.3 \mathrm{MeV}$ above the fission barrier due to its low fissility, which explains the filling of the minimum between the two peaks. Only some neutron-deficient nuclei in the vicinity of ${ }^{180} \mathrm{Hg}$ were measured at energies close to the barrier after beta decay. Considering the measured energy dependence of the structure for ${ }^{201} \mathrm{Tl}$ [70], the fission characteristics of ${ }^{180} \mathrm{Hg}$ and ${ }^{201} \mathrm{Tl}$ are rather similar. Also other nuclei in this mass region show similar features, which have been attributed to the influence of fragment shells [2]. The observation of asymmetric fission in ${ }^{180} \mathrm{Hg}$ has triggered intense experimental and theoretical activity on fission studies with light neutrondeficient nuclei in this mass range. Nuclei in this region are not further considered in this article that concentrates on heavier nuclei with mass numbers $A>200$, which are more important for technical applications.

In the range where asymmetric fission prevails, e.g. from ${ }^{227} \mathrm{Ra}$ to ${ }^{256} \mathrm{Fm}$, the light and the heavy fission-

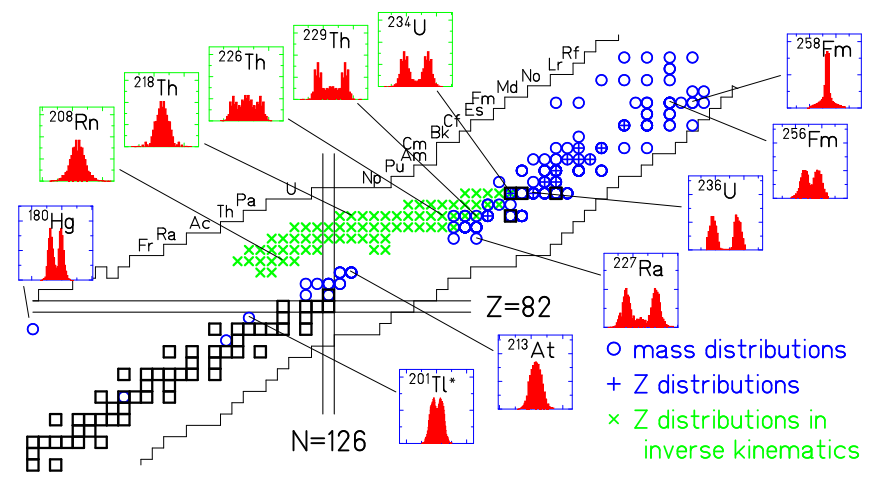

FIG. 9. (Color online) Systematics of fission-fragment distributions. General view on the systems for which mass or nuclear-charge distributions have been measured. The distributions are shown for 12 selected systems. Blue circles (blue crosses): Mass (nuclear-charge) distributions, measured in conventional experiments [70, 71], and references given in 69]. Green crosses: Nuclear-charge distributions, measured in inverse kinematics 69]. The observation of asymmetric fission in ${ }^{180} \mathrm{Hg}$ has triggered very recently an intense experimental activity on fission of neutron-deficient nuclei in this region. These continuously extending cases are not included in the figure.

product components gradually approach each other, see Fig. 9. A quantitative analysis revealed that the mean mass of the heavy component stays approximately constant at about $A=140[73]$. This has been explained by the influence of a deformed $(\beta \approx 0.6)$ fragment shell at $N=88$ [4], suggesting that the position of the heavy fragment is essentially constant in neutron number.

More recent data on $Z$ distributions over long isotopic chains [69], however, reveal very clearly that the position in neutron number varies systematically over more than 7 units, while the position in proton number is approximately constant at $Z=54$ (see Fig. 10). The rather short isotopic sequences covered in former experiments did not show this feature clearly enough and gave the false impression of a constant position in mass. Up to now, it has not been possible to identify the fragment shells, which are behind the observed position of the heavy fragments in the actinides. Although the position of the heavy fragment is almost constant at $Z \approx 54$, it may be doubted that a proton shell is at the origin of the asymmetric fission of the actinides, because a proton shell in this region is in conflict with shell-model calculations [74, 76. Also the sign of the charge polarization suggests that a neutron shell is at the origin of this so-called S2 fission channel, which dominates the asymmetric fission component, as discussed in more detail below.

At present, we limit ourselves in extracting the positions and the shapes of the fission valleys of the standard 1 (S1) and the standard 2 (S2) fission channels (in the nomenclature of Brosa et al. 77]), which form part of 


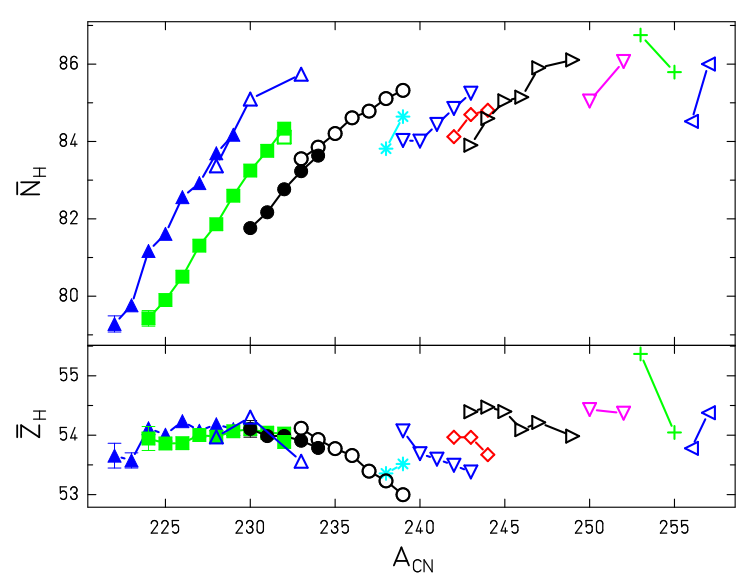

FIG. 10. (Color online) Mean neutron and proton number of the heavy component in asymmetric fission in the actinide region before the emission of prompt neutrons. The values were deduced from measured mass and nuclear-charge distributions using the GEF model for the correction of charge polarization and prompt-neutron emission. Open symbols denote results from conventional experiments, full symbols refer to an experiment with relativistic projectile fragments of ${ }^{238} \mathrm{U}$ [69]. Data points for the same $Z_{C N}$ are connected. (See Tables VIII and IX for references of the underlying experimental data).

the asymmetric fission component, and of the symmetric super-long (SL) channel. In addition, a super-asymmetric (SA) fission channel is considered that has the properties of an extremely asymmetric fission channel, evidenced in the TKE- $A$ (total kinetic energy - mass) distributions of many actinides and named S3 by Mulgin et al. [78]. (In this work we use both names SA and S3 as synonyms to denote the asymmetric channel with the properties of the S3 channel described by Mulgin et al. [78]. Our analysis could not confirm the existence of the separate fission channels SA, S3, nor the SX channel postulated by Brosa et al., see section XI C.) This is done by a fit to the corresponding structures in the measured mass distributions. Eventually, some shells in the complementary fragment are also assumed. The depths of the fission valleys are deduced from the relative yields of the fission channels by assuming that the quantum oscillators in the different fission valleys are coupled, which implies that their populations in the vicinity of the outer barrier are in thermal equilibrium. The potential at this elongation is calculated as the sum of the macroscopic potential, which is a function of the fissioning nucleus, and of the shell effects. The magnitudes of the shell effects are assumed to be the same for all fissioning systems.

Fig. 11 illustrates how the observed transition from symmetric to asymmetric fission around ${ }^{226} \mathrm{Th}$ can be explained by the competition of the macroscopic potential that favors mass-symmetric splits and the shell effect around $Z=55$, even if the shell strength is assumed to be constant. (We stress again that the nearly constant

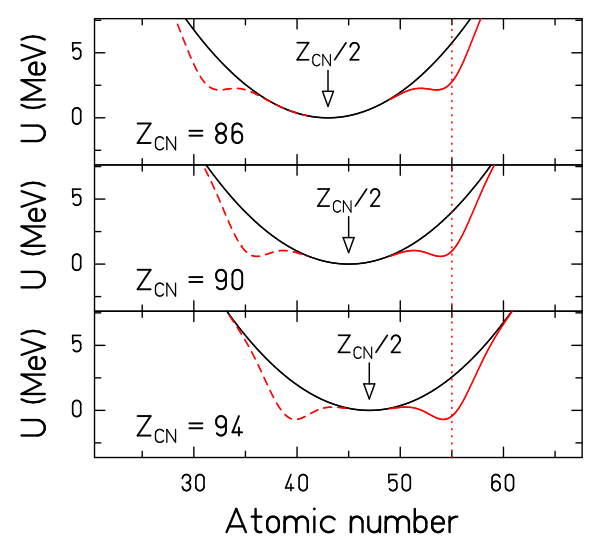

FIG. 11. (Color online) Schematic illustration of the potential energy for mass-asymmetric shape distortions on the fission path, after an idea of Itkis et al. 79]. The black curve shows the macroscopic potential that is minimum at symmetry, while the red curve includes the extra binding due to an assumed shell appearing at $Z=55$ in the heavy fragment. See text for details.

position at $Z=55$ of the asymmetric fission component is deduced from experimental data. We do not claim that it is caused by a proton shell. See also the discussion in other parts of this paper.) With increasing size of the system, the position of the shell assumed to be fixed at $Z$ $=55$ is found closer to symmetry, which is favored by the macroscopic potential. In radium, the potential is lowest at mass symmetry, favoring single-humped mass distributions, in thorium, the potential at symmetry and near $Z=55$ is about equal, creating triple-humped mass distributions, and in plutonium, the potential is lowest near $Z=55$, favoring double-humped mass distributions.

The parametrizations and specific parameter values of the fragment shells actually used in the GEF code are given in Sec. VII.

\section{Charge Polarization}

Besides the mass number, also the numbers of protons and neutrons in a fission fragment can vary independently. In mass-asymmetric fission, the mean $Z / A$ value is found to be smaller on average in the heavy fragment. The cluster plot of a calculation with the GEF code, shown in Fig. 12, demonstrates this finding on a nuclear chart. Fig. 12 shows the yields of the fission fragments of the system ${ }^{235} \mathrm{U}\left(\mathrm{n}_{\mathrm{th}}, \mathrm{f}\right)$ before emission of prompt neutrons. The model reproduces the measured mass-dependent prompt-neutron yields of ${ }^{237} \mathrm{~Np}(\mathrm{n}, \mathrm{f})$ [80] at different incident neutron energies and yields of the light fission fragments after prompt-neutron emission of ${ }^{235} \mathrm{U}\left(\mathrm{n}_{\mathrm{th}}, \mathrm{f}\right)$ [81]. Therefore, this calculation is expected to be quite realistic.

Fig. 13 illustrates how the $Z / A$ degree of freedom is 


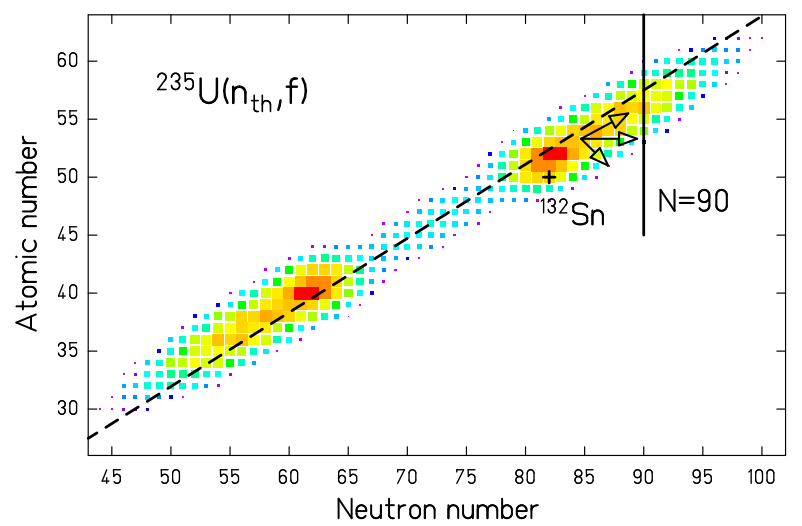

FIG. 12. (Color online) Calculated nuclide distribution produced in thermal-neutron-induced fission of ${ }^{235} \mathrm{U}$ before the emission of prompt neutrons. The size of the clusters and the color scale from cold (blue) to warm(red) represent the yield in a logarithmic scale. The dashed line marks the nuclei with the same $N / Z$ ratio as the fissioning nucleus ${ }^{236} \mathrm{U}$. In addition, the position of the doubly magic ${ }^{132} \mathrm{Sn}$ and an assumed neutron shell at $N=90$ are marked. See text for details.

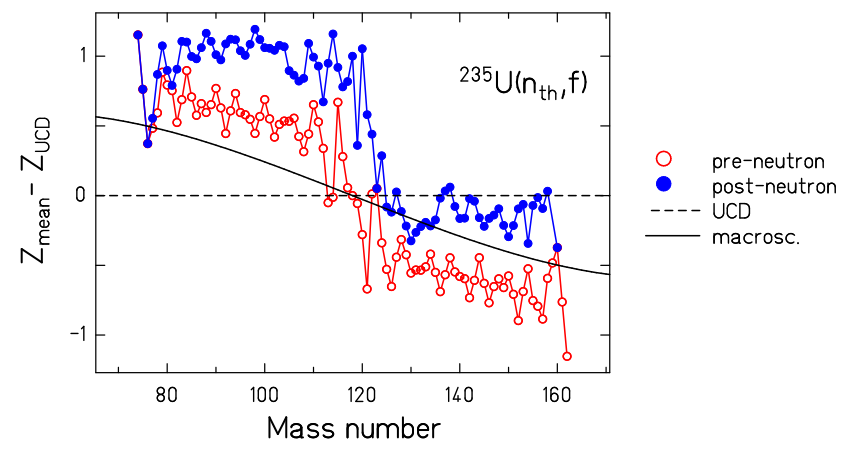

FIG. 13. (Color online) Deviation of the mean nuclear charge of isobaric chains from the UCD value for different cases for the system ${ }^{235} \mathrm{U}\left(\mathrm{n}_{\mathrm{th}}, \mathrm{f}\right)$. Dashed line: $\mathrm{UCD}$ value. Full line: Macroscopic value at scission. Open symbols: Values before prompt-neutron emission as a function of pre-neutron mass. Full symbols: Values after prompt-neutron emission as a function of post-neutron mass.

treated in the GEF model. First, a calculation minimizes the energy of the system near scission with respect to the deformations and the charge densities ( $Z / A$ ratio) of the two fragments without considering structural effects. The macroscopic binding energies of the two fragments and the Coulomb repulsion between the fragments at a tip distance of $1 \mathrm{fm}$ are considered. The neutron loss by emission of prompt neutrons that is consistent with the available data on mass-dependent prompt-neutron yields would not be sufficient to match the $Z_{\text {mean }}-Z_{U C D}$ values of the measured post-neutron nuclide distribution. In order to be consistent with the systematics of mass- dependent prompt-neutron yields, an additional charge polarization of 0.32 units in $Z_{\text {mean }}-Z_{U C D}$ of the preneutron nuclide distribution, essentially constant over the whole mass range, before the prompt-neutron emission, must be assumed. Since it is further assumed that the fission process of all actinides is caused by essentially the same fragment shells, this polarization is expected to be the same in the asymmetric fission channels for all systems.

The fine structure in the curves in Fig. 13 results from the even-odd fluctuations in the fission-fragment yields as a function of atomic number $Z$ and neutron number $N$.

Fig. 12 illustrates the origin of this charge polarization. The additional binding energy of spherical nuclei in the S1 fission channel in the vicinity of the $N=82$ and the $Z=50$ shells increases when approaching the doubly magic ${ }^{132} \mathrm{Sn}$. This explains why the fragments in the S1 fission channel tend to be more neutron-rich than expected from the optimization of the macroscopic energy. The charge polarization of the fragments with deformed shape in the S2 fission channel is explained by the force caused by a shell around $N=90$. This force can be decomposed in a force towards mass asymmetry and a force towards higher $N / Z$ values at constant mass as illustrated in Fig. 12. Since the curvature of the binding energy against charge polarization is much larger than the curvature of the macroscopic potential for mass-asymmetric distortions, the displacement of the mass peak from symmetry $(\Delta A=140-118=22)$ is much larger than the displacement in charge polarization (0.32 units).

This reasoning indicates that the $\mathrm{S} 2$ fission channel is mainly caused by a deformed neutron shell, because a proton shell in the heavy fragment would induce a charge polarization in the opposite direction. Thus, the finding of an almost constant position in atomic number of the asymmetric fission component in the actinides cannot be attributed to a proton shell. It must rather be considered as the result of other influences, e.g. of the competition with the macroscopic potential or additional shells in the light fragment.

\section{E. Quantum Oscillators of Normal Modes}

There is a long tradition in applying the statistical model to nuclear fission [74, 82 84]. However, it is well known [85] that the statistical model, applied to the scission-point configuration, is unable of explaining the variances of the mass and energy distributions and their dependence on the compound-nucleus fissility parameter. Studies of Adeev and Pashkevich [28] suggest that dynamical effects due to the influence of inertia and dissipation can be approximated by considering the properties of the system at an earlier time. That means that the statistical model may give reasonable results if it is applied to a configuration that depends on the typical time constant of the collective coordinate considered.

The potential $U$ in the vicinity of a minimum as a 
function of a collective coordinate $q$ is approximated by a parabola

$$
U=\frac{1}{2} C q^{2} .
$$

Thus, the motion along the collective coordinate $q$ corresponds to an excited state of an harmonic quantum oscillator. In an excited nucleus, there is an exchange of energy between the specific collective and all the other nuclear degrees of freedom that may be considered as a heat bath. In thermal equilibrium, the properties of the heat bath (e.g. state density and temperature $T$ ) determine the probability distribution of excited states of the harmonic oscillator considered. The probability distribution along the coordinate $q$ is the sum of the contributions from different excited states of the oscillator

$$
P(q)=\sum_{0}^{i_{\max }} W_{i}\left|\phi_{i}(q)\right|^{2}
$$

where $W_{i}$ is the population probability of the state $i$ of the oscillator with excitation energy $E_{i}=i \cdot \hbar \omega$, and $\phi_{i}$ is the wave function of that state. The upper limit $i_{\max }$ is given by the available energy of the system. If the temperature of the heat bath does not depend on the energy of the nucleus and if the energy of the nucleus is appreciably higher than the temperature of the heat bath, the probability distribution is a Gaussian distribution with the variance

$$
\sigma_{q}^{2}=\frac{\hbar \omega}{2 C} \operatorname{coth}\left(\frac{\hbar \omega}{2 T}\right) .
$$

Two limiting cases are the width of the zero-point motion

$$
\sigma_{q}^{2}=\frac{\hbar \omega}{2 C}
$$

and the classical limit for $T>>\hbar \omega$

$$
\sigma_{q}^{2}=T / C
$$

The evolution of the width of the mass distribution of the symmetric fission channel at higher excitation energies, where shell effects are essentially washed out, has been the subject of many experimental and theoretical investigations, see Refs. 28, 86 88. It was found that the width of the mass distribution varies with energy $E$ according to the relation $\sigma_{q}^{2}=\frac{\sqrt{E / a}}{C}$ that corresponds to the classical limit with the temperature defined by the Fermi-gas nuclear level density. The values and the variation of the stiffness parameter $C$ as a function of $Z^{2} / A$ agree with theoretical estimations of the stiffness for mass-asymmetric distortions for a configuration between saddle and scission [88].

A more refined consideration is needed for understanding the mass distribution of a fission channel in asymmetric fission at energies little above the fission barrier. Here, the fission valley is formed by a shell effect, which also influences the level density. The restoring force $F(q)$ of the corresponding oscillator is defined by the variation of the entropy $S$

$$
F(q)=T \frac{d S}{d q},
$$

and the effective potential $U(q)$ is obtained by integration

$$
U(q)=\int F(q) d q .
$$

The stiffness $C$ in the vicinity of the potential minimum is given by the second derivative

$$
C=d^{2} U / d q^{2} .
$$

In Fig. 14, the measured increase of the standard deviation of the mass distribution of the asymmetric fission channels (standard 1 and standard 2) 89] is compared with the result of a numerical calculation on the basis of the level-density description presented in Sec. III B

The situation is again different for the chargepolarization degree of freedom. Here, the potential is dominated by the macroscopic contribution. In addition, the zero-point energy is so high that the quantum oscillator is not excited in low-energy fission [90, 91]. Therefore, the width of the charge polarization is essentially a constant value. That does not mean that the observed width of the isotopic or isobaric distributions is a constant, because it is broadened by neutron evaporation.

In the GEF code, the widths of the corresponding observables are described with analytical expressions that represent the physics ideas described above.

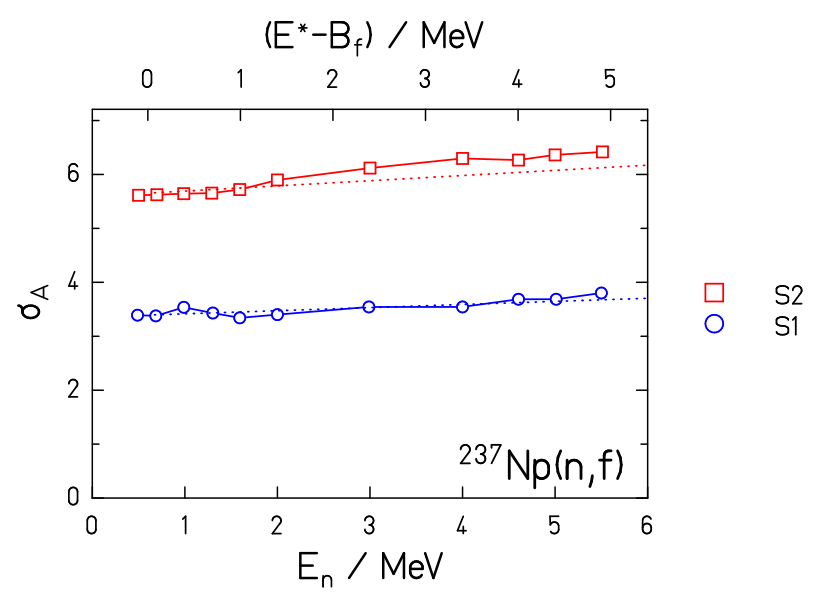

FIG. 14. (Color online) Standard deviation of the mass distribution of the asymmetric fission channels (standard 1 and standard 2) in the fission of ${ }^{237} \mathrm{~Np}(\mathrm{n}, \mathrm{f})$ as a function of the neutron energy $E_{n}$ (lower scale) and the excitation energy above the outer barrier (upper scale). The measured data [89] (symbols) are compared with the calculated widths of the corresponding quantum oscillators (dotted lines). 


\section{F. Energetics of the Fission Process}

In low-energy fission, the available energy, consisting of the $\mathrm{Q}$ value of the reaction plus the initial excitation energy of the fissioning nucleus, ends up either in the total kinetic energy (TKE) or the total excitation energy (TXE) of the fragments. Moreover, the TXE is divided between the two fission fragments. In the GEF code, these values are not parametrized directly. In accordance with the general character of the model, the exchange of the available energy between the different degrees of freedom of the fissioning system is described along the fission path. This way, a consistent and complete description of all phenomena is obtained that depend on the energetics of the fission process. A schematic presentation of the evolution of the fissioning system is shown in Fig. 15.

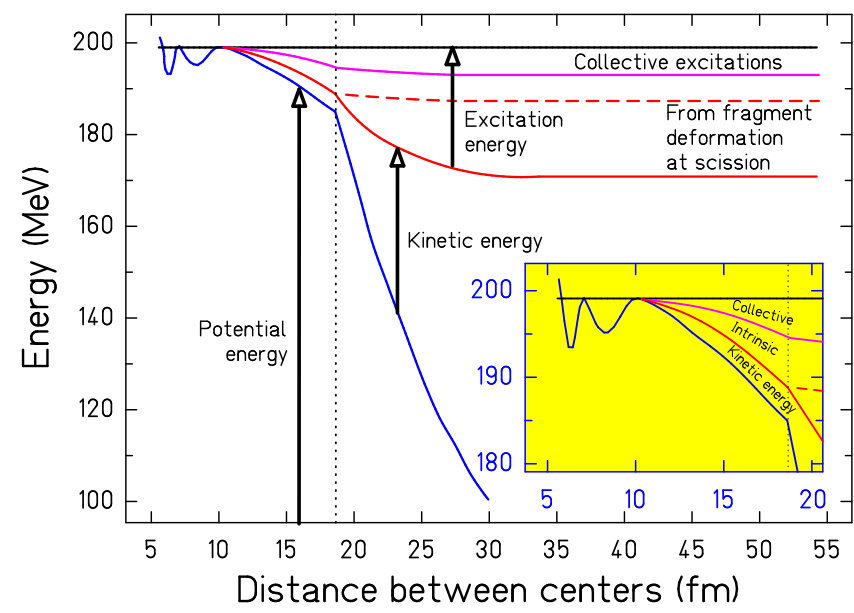

FIG. 15. (Color online) Energetics of the fission process. Schematic presentation of the different energies appearing in the fission process. The vertical dotted line indicates the scission point. The inset illustrates that the energy release due to the decreasing potential energy is partly dissipated into excitations of collective normal modes and intrinsic excitations. The remaining part feeds the pre-scission kinetic energy. The main figure demonstrates that the excitation energy of the fragments still increases right after scission, because the excess surface energy of the deformed fragments with respect to their ground states becomes available. Later, also the collective excitations are damped into the intrinsic degrees of freedom. The figure represents the fission of ${ }^{236} \mathrm{U}$ with an initial excitation energy equal to the fission-barrier height.

\section{From Saddle to Scission}

It is assumed that the energy available above the outer saddle (initial excitation energy of the fissioning nucleus minus the height of the outer fission barrier) is thermalized [20]. This implies that the available energy above the fission barrier is equally shared between each of the different degrees of freedom. Except for the energy range very close to the barrier, most of the available states are intrinsic excitations. Thus, most of the excitation energy available above the outer saddle is stored in intrinsic excitations.

According to two-center shell-model calculations, documented in Fig. 12 of Ref. [25], which is reproduced in Fig. 3 of the present work, there are many level crossings on the first section behind the outer saddle. Due to these level crossings, part of the potential-energy release in this section is transformed into intrinsic excitations by dissipation. This is also true at low excitation energies in the regime of strong pairing correlations, although the situation is more complex [92, 93]. Also this excitation-energy gain is expected to be homogeneously distributed within the nuclear volume.

Further down on the way to scission, the level structure changes only little, the levels almost do not cross any more, dissipation almost vanishes, and the intrinsic excitation energy stays nearly constant. As already discussed in Sec. IID, this change of the single-particle structure indicates a transition in the vicinity of the outer saddle from the mono-nuclear to the di-nuclear regime, where the properties of the individual fission fragments are already well established. This transition can also be observed in the pairing correlations [95] and the congruence energy 96 .

Since both the deformation at the macroscopic barrier and the gain of potential energy from saddle to scission are related with the Coulomb parameter $Z^{2} / A^{1 / 3}$, the amount of dissipated energy from the outer saddle to scission is assumed to be a constant fraction of the calculated macroscopic potential energy gain from saddle to scission 94]. Additional intrinsic excitations that may appear at neck rupture are not considered.

The system enters the di-nuclear regime with an excitation energy $E_{t o t}$ that is equal to the intrinsic excitation energy above the outer saddle plus the energy acquired by dissipation on the first section behind the outer saddle. It is assumed that $E_{\text {tot }}$ is initially shared among the nascent fragments according to the ratio of their masses.

In the di-nuclear regime, the fissioning system consists of two well-defined nuclei in contact through the neck. We assume that the system then evolves on the way to scission towards a state of statistical equilibrium, the macrostate of maximum entropy, where all the available microstates have equal probability [34]. This means that the fission process is slow enough that the dynamical time in the di-nuclear regime is sufficiently long that statistical equilibrium is established. This implies that the intrinsic excitation energy is distributed among the two nascent fragments according to the probability distribution of the available micro-states which is given by the total nuclear level density [97].

Thus, the distribution of excitation energy $E_{1}$ of one fragment at scission is calculated by the statistical weight of the states with a certain division of excitation energy 
between the fragments

$$
\frac{d N}{d E_{1}} \propto \rho_{1}\left(E_{1}\right) \cdot \rho_{2}\left(E_{t o t}-E_{1}\right)
$$

Note that $\rho_{1}$ and $\rho_{2}$ are the level densities of the fragments in their shape at scission, not in their ground-state shape. The remaining energy $E_{t o t}-E_{1}$ is taken by the other fragment.

In the regime of pairing correlations, where the level density was found to grow almost exponentially with increasing excitation energy [98 105], energy sorting will take place, and the light fragment will transfer essentially all its excitation energy to the heavy one [35, 106]. At higher energies, in the independent-particle regime where pairing correlations die out, there is a gradual transition to a division closer to the ratio of the fragment masses according to the validity of the Fermi-gas level density.

The phenomenon of energy sorting explains in a straightforward and natural way the finding of Ref. [80] demonstrated in Fig. 16 that the additional energy introduced in neutron-induced fission of ${ }^{237} \mathrm{~Np}$ raises the neutron multiplicities of the heavy fragment, only. A similar result was reported for the system ${ }^{235} \mathrm{U}(\mathrm{n}, \mathrm{f})$ [107], but data of this kind with good quality are scarce.

Part of the energy gain from saddle to scission may also be transferred to collective modes perpendicular to the fission direction (normal modes [108]). These excitations correspond to correlated motions of the whole system. The division between the fragments depends on the kind of collective motion. As an approximation, it is assumed that the collective excitation energy is equally shared between the fragments.

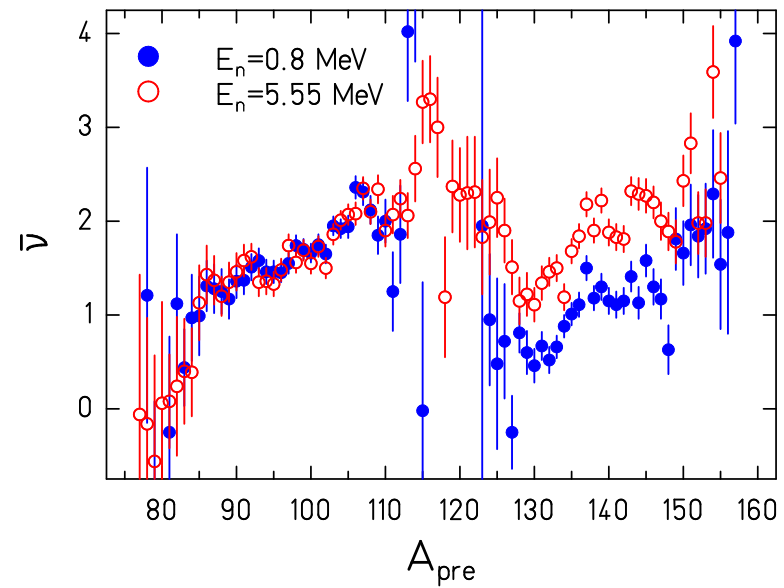

FIG. 16. (Color online) Prompt-neutron multiplicity as a function of the pre-neutron fragment mass for the system ${ }^{237} \mathrm{~Np}(\mathrm{n}, \mathrm{f})$ for $E_{n}=0.8 \mathrm{MeV}$ and $5.55 \mathrm{MeV}$ [80].

\section{Fully Accelerated Fragments}

There is widespread agreement that the saw-tooth shape of the prompt- neutron yields (see Fig. 16) is caused by the deformation energies of the nascent fragments at scission. The scission-point model of Ref. 74 attributes it to the influence of fragment shells, the random-neckrupture model [77] links it to the location of the rupture, and also microscopic calculations predict large deformation energies of the fragments near scission [109]. Large even-odd effects in the fragment $Z$ distributions indicate that the intrinsic excitation energy at scission is generally much too low to account for the variation of the prompt-neutron yield by several units over the different fragments.

In the scission-point model of Ref. [74], the deformation at scission is determined by minimizing the potential energy for fixed tip distance. An alternative condition would be to require a fixed distance between the centres of mass of the two nascent fragments. The validity of one or the other case depends on the magnitude of dissipation [110]. The first case is valid in the presence of strong dissipation, because the relative velocity of the fragments is slowed down by an attractive force which acts on the nascent fragments through the neck. The second case is valid for weak dissipation, where the relative velocity of the fragments reflects the action of the long-range Coulomb force between the nascent fragments. The magnitude of dissipation in low-energy fission in the regime of strong pairing correlations is a delicate problem [93]. The GEF model follows the idea of Ref. 74. In this scenario, the macroscopic forces favor fragments that are strongly deformed $(\beta \approx 0.5$ to 0.6 ). Thus, shell effects at these large deformations are favored, while e.g. the influence of the ${ }^{132} \mathrm{Sn}$ spherical shell is weakened by the macroscopic forces. According to Ref. 74, this explains the weak relative yield of the standard 1 fission channel corresponding to spherical heavy fragments in the vicinity of ${ }^{132} \mathrm{Sn}$, while the bulk of the yield of the asymmetric component is provided by the standard 2 fission channel with appreciably more strongly deformed heavy fragments.

In the GEF model, the mean deformation and the width of the different fragment shells that correspond to the different fission valleys are determined empirically by the deformation distribution of the light and the heavy fragment that is consistent with the observed promptneutron multiplicity distribution.

After fixing the intrinsic and the collective excitation energy at scission as well as the fragment deformation energy, the total kinetic energy is determined by energy conservation for a given $A$ and $Z$ split that defines the fission $\mathrm{Q}$ value.

The calculated two-dimensional $A-E_{k i n}$ distribution and the TKE distribution for the system ${ }^{235} \mathrm{U}\left(\mathrm{n}_{\mathrm{th}}, \mathrm{f}\right)$ are shown in Figs. 17 and 18. Fig. 18 demonstrates that the numerical result of the GEF model is not well represented by a normal distribution. Deviations of measured TKE distributions from a normal distribution have al- 


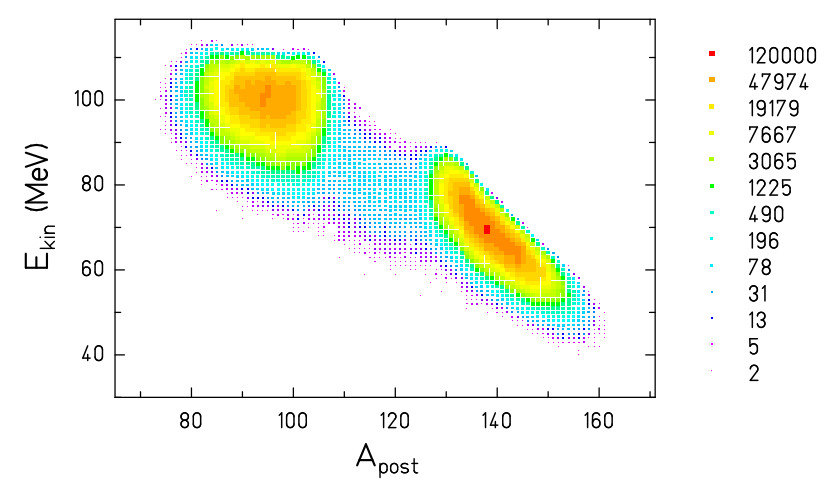

FIG. 17. (Color online) Calculated 2-dimensional $A-E_{k i n}$ distribution for the system ${ }^{235} \mathrm{U}\left(\mathrm{n}_{\mathrm{th}}, \mathrm{f}\right)$. The color scale gives the number of events per bin.

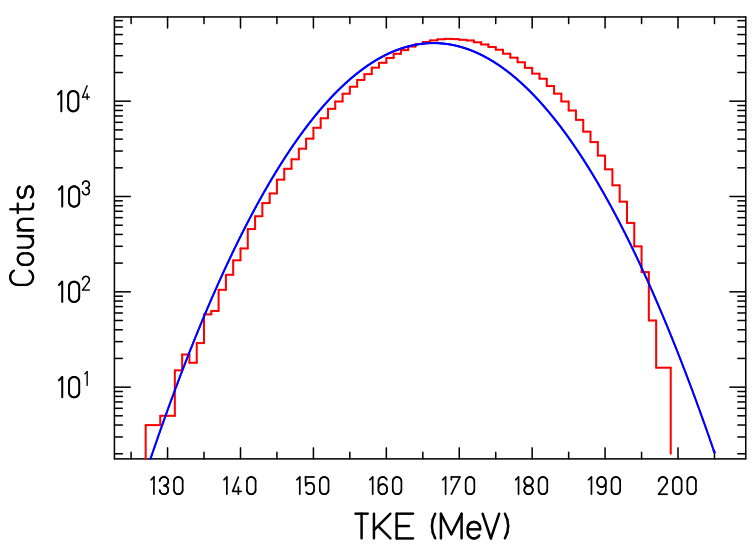

FIG. 18. (Color online) Calculated post-neutron TKE distribution for the system ${ }^{235} \mathrm{U}\left(\mathrm{n}_{\mathrm{th}}, \mathrm{f}\right)$ (histogram). The smooth curve shows a fit with a normal distribution.

ready been described by Brosa et al. [77] and Zhdanov et al. 111] by adapted analytical formulations.

\section{G. Even-odd Effects}

Several quantities show a systematic staggering for fragments with even or odd number of protons. The most striking effect is the enhanced production of even- $Z$ fragments, for which values up to $40 \%$ in the thermalneutron-induced fission of ${ }^{232} \mathrm{Th}$ have been found. But also the yields of even- $N$ fragments are found to be systematically higher. Moreover, the total kinetic energies of fragments in even- $Z$ charge splits from even- $Z$ fissioning systems were found to be enhanced.

\section{Z Distribution}

The enhanced production of even- $Z$ fragments is the most direct observation, because the number of protons in the fragments is generally not changed after scission since the probability for proton evaporation from the neutronrich fission fragments is very low.

In the quasi-particle picture, the ground state is the only state in an even-even nucleus that is systematically lower than the states in an odd-odd nucleus on an absolute energy scale. The case of an odd-mass nucleus is in between. Therefore, we attribute the observed even-odd effect in fission fragment $Z$ distributions to the population of the ground state or eventually some collective states in the vicinity of the ground state of even- $Z$ fragments. It seems straightforward to attribute the observed enhanced production of even- $Z$ light fragments [112] to the energy-sorting mechanism [113] that explained already the differential behavior of the prompt-neutron yields [35]. If the time until scission is sufficiently long for the energy sorting to be accomplished, the system can still gain an additional amount of entropy by predominantly producing even-even light fragments. Compared to the production of odd-odd light fragments, the excitation energy of the heavy fragment increases by two times the pairing gap, and its entropy increases due to the increasing number of available states.

The quantitative calculation of the even-odd effect is based on the assumption that the distribution of excited states in the two fragments at scission is in statistical equilibrium. This means that each state of the fissioning system is populated with the same probability.

For an even-even fissioning nucleus, the number of configurations with $Z_{1}$ even at fixed total reduced energy $U_{\text {tot }}$ is given by

$$
\begin{array}{r}
N_{Z_{1}=e}^{e e}\left(Z_{1}\right)= \\
\int_{-2 \Delta_{1}}^{U_{t o t}+2 \Delta_{2}} \rho_{1}\left(U_{1}\right)_{(e e)} \rho_{2}\left(U_{t o t}-U_{1}\right)_{(e e)} d U_{1}+ \\
\int_{-\Delta_{1}}^{U_{t o t}+\Delta_{2}} \rho_{1}\left(U_{1}\right)_{(e o)} \rho_{2}\left(U_{t o t}-U_{1}\right)_{(e o)} d U_{1}
\end{array}
$$

where $\rho_{i}\left(U_{i}\right)_{(e e)}$ and $\rho_{i}\left(U_{i}\right)_{(e o)}$ are the level densities of representative even-even and even-odd nuclei, respectively, with mass close to $A_{1}$ or $A_{2}$. The reduced energy $U$ is shifted with respect to the energy $E$ above the nuclear ground state by $U=E-n \Delta, n=0,1,2$ for odd-odd, odd-mass, and even-even nuclei, respectively.

The number of configurations with $Z_{1}$ odd for an even- 
even fissioning nucleus is

$$
\begin{gathered}
N_{Z_{1}=o}^{e e}\left(Z_{1}\right)= \\
\int_{-\Delta_{1}}^{U_{t o t}-\Delta_{2}} \rho_{1}\left(U_{1}\right)_{(o e)} \rho_{2}\left(U_{t o t}-U_{1}\right)_{(o e)} d U_{1}+ \\
\int_{0}^{U_{t o t}} \rho_{1}\left(U_{1}\right)_{(o o)} \rho_{2}\left(U_{t o t}-U_{1}\right)_{(o o)} d U_{1},
\end{gathered}
$$

where $\rho_{i}\left(U_{i}\right)_{(o)}$ and $\rho_{i}\left(U_{i}\right)_{(o o)}$ are the level densities of representative odd-even and odd-odd nuclei, respectively, with mass close to $A_{1}$ or $A_{2}$. The yield for even- $Z_{1}$ nuclei is $Y_{Z_{1}=e}^{e e}\left(Z_{1}\right)=N_{Z_{1}=e}^{e e}\left(Z_{1}\right) / N_{\text {tot }}^{e e} /\left(Z_{1}\right)$ with $N_{\text {tot }}^{e e}\left(Z_{1}\right)=N_{Z_{1}=e}^{e e}\left(Z_{1}\right)+N_{Z_{1}=o}^{e e}\left(Z_{1}\right)$. Similar equations hold for odd-even, even-odd and odd-odd fissioning systems. The total available reduced intrinsic excitation energy $U_{\text {tot }}$ is assumed to be a fraction of the potentialenergy difference from saddle to scission plus the initial excitation energy above the barrier. Thus, it increases with the Coulomb parameter $Z^{2} / A^{1 / 3}$ of the fissioning nucleus, according to Sec. III F.

This approach reproduces the observed salient features of the even-odd effect [112]: (i) The global even-odd effect $\left(\Sigma Y_{Z=e}-\Sigma Y_{Z=o}\right) /\left(\Sigma Y_{Z=e}+\Sigma Y_{Z=o}\right)$ decreases with the Coulomb parameter $Z^{2} / A^{1 / 3}$ and with increasing initial excitation energy. (ii) The local even-odd effect

$\delta_{p}(Z+3 / 2)=1 / 8(-1)^{Z+1}(\ln Y(Z+3)-\ln Y(Z)-$ $3[\ln Y(Z+2)-\ln Y(Z+1)])$

increases towards mass asymmetry. (Note that this equation is only defined for integer $Z$ values!) (iii) The local even-odd effect for odd- $Z$ fissioning nuclei is zero at mass symmetry and approaches the value of even- $Z$ nuclei for large mass asymmetry.

In the GEF code, an analytical function that parametrizes the result of this approach with some adjustment to the measured values is used.

\section{2. $N$ Distribution}

In the GEF model it is assumed that the even-odd effect in the yields of the fission fragments as a function of the atomic number $Z$ is also present in the yields as a function of the number of neutrons $N$. However, this structure cannot easily be observed. It is washed away by prompt-neutron emission, and another even-odd structure is established. This structure is generated by the influence of the neutron separation energy on the last stages of the evaporation process. It has also been observed in the cross-section of projectile fragments in reactions at relativistic energies 114 116].

An interesting feature of the even-odd staggering of the fission-product yields in neutron number is that the structure generated by the evaporation process is not sensitive to the excitation energy of the fragments: The structure will remain unchanged with increasing excitation energy.
That is in contrast to the even-odd staggering in atomic number.

However, the pairing gap depends on the angular momentum, and the pairing correlations gradually disappear around an angular momentum $J=15 \hbar$. Thus, the evenodd staggering in the neutron binding energy and also the even-odd staggering in neutron number will decrease accordingly with increasing fragment angular momentum. The angular-momentum dependence of the pairing gap is considered in the GEF code according to Ref. [117] as

$$
\Delta=\Delta_{(J=0)} \cdot \sqrt{1-\frac{J}{J_{c r i t}}} .
$$

\section{Total Kinetic Energy}

The global even-odd effect in the $Z$ distribution of the fission-fragment yields at constant TKE (or at constant kinetic energy of the light fragment) increases towards higher energy and decreases towards lower energy. This finding can also be expressed in a different way: The TKE distributions of even- $Z$ elements are shifted to higher values with respect to their odd- $Z$ neighbors. The magnitude of this shift for thermal-neutron-induced fission of ${ }^{229} \mathrm{Th},{ }^{233} \mathrm{U},{ }^{235} \mathrm{U},{ }^{239} \mathrm{Pu},{ }^{241} \mathrm{Pu},{ }^{245} \mathrm{Cm}$, and ${ }^{249} \mathrm{Cf}$ is correlated with the global even-odd effect in the $Z$ yields. This can be deduced from the slope of the even-odd effect in the $Z$ yields as a function of the kinetic energy of the light fragment shown in Fig. 13 of Ref. [118]. In the GEF model, the even-odd fluctuation of the TKE is calculated by a simple description following an idea of Ref. [81]. It is assumed that two components contribute to the even- $Z$ yield. One component which contains at least one broken proton pair and the other where no proton pair is broken. If one proton pair is broken anywhere between the saddle and the scission point, it is assumed that the two unpaired protons will be distributed statistically on the two fragments. Therefore, the even- $Z$ yield component with at least one broken pair is equal in amplitude to the odd- $Z$ yield, and the energy distributions are expected to be the same, too. In contrast, the superfluid component of the even- $Z$ yield is shifted to higher kinetic energies. The shapes of the energy distributions of the two components of the even- $Z$ yields are assumed to be equal. The shift between the two components is the only free parameter of this description. The data are well described if the two components are assumed to be shifted by $1.7 \mathrm{MeV}$.

This picture is valid for the situation for nearsymmetric mass splits. The increase of the even-odd effect in $Z$ yields with growing asymmetry has no influence on the magnitude of the even-odd effect in total kinetic energy, because it is ascribed to an accumulation of unpaired nucleons in the heavy fragment, not to a reduction of pair breaking. 


\section{H. Spontaneous Fission}

Fission from excitation energies above or in the vicinity of the fission barrier and spontaneous fission, starting from the nuclear ground state, are very much related. Therefore, both processes must be described on a common footing. The potential-energy surface is the same [119]. The most important difference is that the passage across the barrier from the entrance point to the exit point, where the height of the potential exceeds the available excitation energy, is only possible by tunneling in spontaneous fission.

In the GEF model, it is assumed that the exit point is located inside one of the fission valleys. Therefore, the relative yields of the fission channels are given by the relative values of the transmission coefficients, corresponding to the different fission channels.

For covering fission from energies above and below the height of the fission barrier, Eq. (2) was extended in order to account for tunneling. The relative yield of a specific fission channel $i$ is calculated with the following equation

$$
Y_{i}=\frac{e^{E_{B, i}^{*} / T}}{1+e^{\left(-E_{B, i}^{*} /\left(T \cdot T_{t u n}^{i} /\left(T-T_{\text {tun }}^{i}\right)\right)\right)}},
$$

which asymptotically gives the same relative yields as Eq. (2) for energies well above the barrier, if the relevant potential energies are approximated by their values at the second barrier. The influence of the excitation energy $E_{B, i}^{*}$ relative to the second-barrier height $B_{B, i}$ on the relative yield of fission channel $i$ is assumed to be well described by an exponential function with the slope parameter $T_{\text {tun }}^{i}$. Note that $E_{B, i}^{*}$ is negative in the case of spontaneous fission!

The potential energy along the fission path for ${ }^{240} \mathrm{Pu}$ is schematically shown in Fig. 19. In addition to the

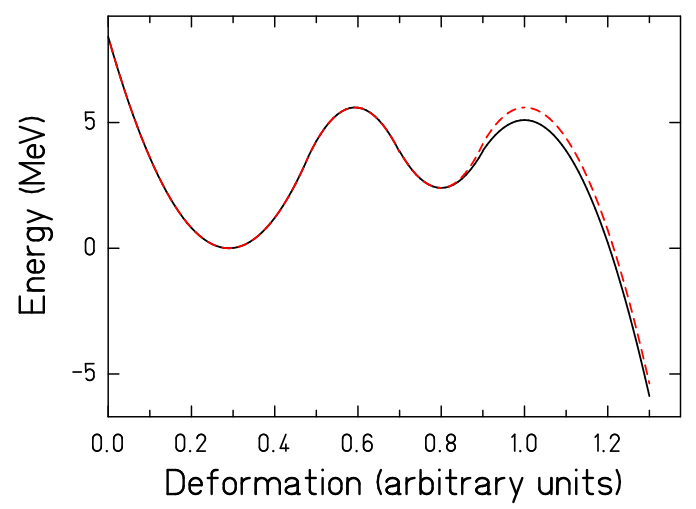

FIG. 19. (Color online) Schematic drawing of the potential energy as a function of deformation in fission direction. The heights of the inner and the outer barrier as well as the depth of the second minimum are those determined for ${ }^{240} \mathrm{Pu}$ 57 (full line). The potential for another fission path with a 0.5 $\mathrm{MeV}$ higher outer barrier is shown in addition (dashed line). passage over the lowest outer barrier, a second passage over another, $0.5 \mathrm{MeV}$ higher, outer barrier of another fission channel is schematically shown.

By the systematics of spontaneous-fission half lives it is known that a variation of the binding energy of the fissioning nucleus (e.g. by a variation of the shell-correction energy in the ground-state) by $1 \mathrm{MeV}$ changes the fission half life by about 5 orders of magnitude [121, 122]. In that case, the energy deficit is modified over the whole path from entrance point to exit point. In the present case, it is only the potential around the outer barrier and beyond that is modified by the influence of fragment shells. Therefore, the relative population of the different fission valleys is much less sensitive to the magnitudes of the shell effects in the different fission valleys than the spontaneous-fission half-life is to the ground-state shell effect. A simple estimate for this sensitivity is given by the Hill-Wheeler formula, using the $\hbar \omega$ value of the outer barrier, which is in the order of $0.7 \mathrm{MeV}$ [57, 123]. (We do not consider the appreciably larger value found for symmetric fission in Ref. [123], which has a very low yield.) However, Fig. 19 illustrates that the sensitivity is expected to be much weaker, because the potential towards the second minimum is not affected by the shell effects that form the different fission valleys.

The sensitivity of the transmission coefficient to the influence of the shell effect that forms the fission valley is parametrized with the Hill-Wheeler formula with a $\hbar \omega$ value around $2 \mathrm{MeV}$, corresponding to $T_{\text {tun }}^{i} \approx 0.3$ $\mathrm{MeV}$, which is determined by a fit to the experimental fission-fragment yields in the different fission channels. In view of the above reasoning, this value that is even much larger than the value governing the tunneling through the outer barrier appears to be reasonable. A theoretical estimation of the difference of the transmission for different fission channels would require a full dynamical quantum-mechanical calculation of the problem, which is not available with the necessary precision.

Another aspect in which the initial excitation energy of the fissioning nucleus matters is the energetics of the fission process. The excitation of the quantum oscillators for mass-asymmetric distortions is considered by a variation of the effective temperature. As mentioned in Sec. III E, the fluctuation of the charge polarization is not expected to vary, because this mode is not excited in lowenergy fission anyhow. A variation of the intrinsic excitation energy at scission is expected to influence the magnitude of the even-odd effect in $Z$ yields, see Sec. III G Unfortunately, for spontaneous fission this cannot be confirmed by experiment, because such kind of data are not available.

The description developed for the case of spontaneous fission is also used when the initial excitation energy falls below the height of the outer fission barrier of a specific fission channel. The only necessary modification to be considered is the finite initial energy of the system above the nuclear ground state. 


\section{Fission-fragment Angular Momentum}

The empirical information on fission-fragment angular momenta is mostly obtained from the relative yields of fission fragments in their isomeric states, from the multiplicity and angular distributions of prompt gamma rays, and from the spectroscopy of the gamma transitions between yrast states. Methods for deducing the initial angular-momentum distributions from these data rely on the modeling of the statistical decay of the excited fragments by neutron emission and gamma de-excitation. In particular, the angular momentum carried away by the neutrons and the gammas before reaching the isomer must be estimated [124 -126]. The analysis of the gamma multiplicity requires the distinction of statistical (dipole) and rotational or vibrational (quadrupole) radiation [127].

The mechanism that is responsible for creating the angular momenta of the fission fragments has long been controversially discussed. According to Ref. [128], the thermal excitation of angular-momentum-bearing modes [86, 129, 130] within the statistical model requires temperatures as high as 2 or $3 \mathrm{MeV}$ that might only be possible if strong coupling between collective degrees of freedom and weak coupling to intrinsic degrees of freedom is assumed. This is in contradiction to large single-particle excitations found in near magic nuclei [131]. The pumping of fragment angular momenta by the zero-point motion of these modes has been found to explain the measured values 129, 132], however with the exception of spherical fragments in the vicinity of the doubly magic ${ }^{132} \mathrm{Sn}$. Also, the torque by electrostatic repulsion between deformed fragments at scission has been considered [129, 133].

Recently, Kadmensky came up with another idea that seems to solve these problems: He pointed out that the assumption, often implicitly made, that the orbital angular momentum of the fission fragments is essentially zero, is in conflict with the uncertainty principle 134]. He assumes that the fluctuation of the orbital angular momentum according to the quantum-mechanical uncertainty is the true principal origin of the fission-fragment angular momenta. The orbital angular momentum is compensated by the fragment angular momenta, eventually also with single-particle excitations in spherical nuclei. Thus, the angular momenta of the two fragments are strongly aligned. The angular momentum $J_{1}+J_{2}$ is shared between the two fragments according to the ratio of their momenta of inertia at scission $I_{1}$ and $I_{2}$ in order to minimize the energy expense $E_{r o t}=J_{1}^{2} /\left(2 I_{1}\right)+J_{2}^{2} /\left(2 I_{2}\right)$.

In the GEF code, we are primarily interested in providing the information that is relevant for technical applications. This is the intensity and the spectral distribution of the gamma radiation, including the population of isomeric states. (GEF does not cover the delayed emission of gammas and neutrons. Dedicated codes have been developed for this purpose, and they can be fed with the yields provided by the GEF code.) For this purpose it is sufficient to know the angular-momentum distribution after the emission of statistical gammas, because it determines the energy that goes into collective E2 photons and, approximately, the angular-momentum-dependent effective threshold for neutron emission. This distribution can rather directly be deduced from measured yields of spin isomers without considering the angular momentum carried away by the emission of prompt neutrons and statistical gammas, if one assumes that all collective transitions pass by a sequence of states on the yrast line. Nota bene this distribution is not identical to the angularmomentum distribution of the primary fragments before prompt-neutron and gamma emission, but it is close to it [124, 125].

The shape of the angular-momentum distribution of a fragment is assumed to be given by the formula

$$
N(J) \propto(2 J+1 \hbar) \exp \left(-\frac{J(J+1 \hbar)}{2 b^{2}}\right),
$$

which describes the angular-momentum distribution of a single object that is in equilibrium with a heat bath of temperature $T_{\text {eff }}$. The spin cut-off parameter $b$ is related to the rms spin value by $J_{r m s}=b \cdot \sqrt{2}$ that is given by

$$
J_{r m s}=\sqrt{2 I T_{\text {eff }}} .
$$

We found that the measured isomeric ratios are well reproduced by assuming

$$
T_{\text {eff }}=T_{n u c},
$$

where the intrinsic nuclear temperature $T_{n u c}$ is defined by the inverse logarithmic slope of the energy-dependent nuclear level density according to the parametrization presented in Sec. III B. This would be in agreement with a scenario, where the fragment angular momentum is created by nuclear excitations in the nascent fragments in the scission configuration. It is essential that a realistic temperature in the regime of pairing correlations is used. Below the critical pairing energy, this value is appreciably larger than the temperature deduced from the Fermi-gas expression.

Thus, the formulation used in GEF corresponds to the statistical population of any available state in the nascent fragments according to the nuclear temperature at scission. The influence of the orbital angular momentum on the creation of fragment angular momentum is not explicitly considered, although this idea of Kadmensky is very appealing. This question needs further clarification by dedicated experimental and theoretical studies.

Starting from the rigid-body momentum of inertia of a fragment with mass number $A_{f}$ and deformation $\alpha$ at scission 135]

$$
\begin{array}{r}
I_{\text {rigid }}=1.16^{2} \cdot A_{f}^{5 / 3} /\left(103.8415 \mathrm{MeV} / \hbar^{2}\right) \\
\cdot\left(1+1 / 2 \cdot \alpha+9 / 7 \cdot \alpha^{2}\right) .
\end{array}
$$

The reduction of $I$ in the pairing regime [136]

$$
I=I_{\text {rigid }} \cdot\left(1-0.8 \cdot \exp \left(-0.693 E_{\text {exc }} /(5 \mathrm{MeV})\right)\right)
$$


is considered as a function of excitation energy $E_{e x c}$.

The initial spin distribution of the fissioning nucleus is included by adding the rms value $J_{C N}$ in a classical approximation

$$
J_{r m s}=\sqrt{2 I T_{e f f}+J_{C N}^{2}} .
$$

In spontaneous fission, $J_{C N}$ is the ground-state spin $J_{0}$. In induced fission, it is the value of the entrance channel. Thus, in neutron-induced fission, the influence of the spin and the orbital angular momentum of the incident neutron (center-of-mass energy $E_{c m}$ ) and the spin of the target nucleus $J_{0}$ is given by

$$
\sqrt{\frac{J_{C N}}{\hbar}=}=
$$

Finally, the observed enhancement of the angular momenta of odd- $Z$ fission fragments [137 is considered by increasing $J_{r m s}$ for odd-Z fragments by the amount $F_{\text {odd }} \cdot A_{f}^{2 / 3}$, which depends on the fragment mass $A_{f}$ in accordance with the average single-particle spin at the Fermi level that is associated with the enhanced angularmomentum in odd-Z fragments in Ref. [138]. The only parameter of this description is $F_{\text {odd }}=0.0148$, which was adjusted to reproduce the experimental isomeric ratios. With this value, we obtain that the deduced magnitude of the odd-even staggering in fission-fragment angular momenta (about 0.4 units in $\hbar$ ) is appreciably smaller than the one extracted by Naik et al. 126] (about 3 to 4 units of $\hbar$ ) and the one theoretically estimated by Tomar et al. [138] (about 2 units of $\hbar$ ). It seems that the odd-even staggering is considerably amplified by the correction for neutron and statistical gamma emission performed in Ref. [126].

For a given angular-momentum distribution, the population of the states of interest on the yrast line is calculated with a modified sharp-cut-off model, taking into account the energy difference between the states. That means that the state of interest is fed by the angularmomentum range in direction of increasing rotational energy up to the next state of interest. The state with the highest, respectively lowest, angular momentum collects the remaining angular-momentum range below, respectively above, that state. Best reproduction of the measured isomeric ratios was obtained by "cutting" the angular-momentum range between two states of interest with spin values $J_{1}$ and $J_{2}$ and energy values $E_{1}$ and $E_{2}$ at

$$
J_{12}^{\text {cut }}=J_{1}+\Delta J_{12} \cdot\left(\frac{1}{2}+\frac{1}{2} \cdot \frac{\Delta E_{12} / \Delta J_{12}}{\left|\Delta E_{12} / \Delta J_{12}\right|+\frac{0.05 M e V}{\hbar}}\right)
$$

with $\Delta J_{12}=J_{2}-J_{1}$ and $\Delta E_{12}=E_{2}-E_{1}$. This way $J_{12}^{\text {cut }}$ moves towards the middle between the two spin values, when the energy difference tends to zero.
The population of states at high angular momentum is limited by the condition that the available excitation energy of the system at scission is sufficiently high.

This procedure substantially differs from the often used descriptions of Madland and England [146] and of Rudstam [147]. Madland and England do not consider the preferred direction of the E2 transitions towards lowerlying states. Rudstam emphasizes the change of the fragment spin due to E1 radiation, which is in conflict with Refs. 124, 125], and in our opinion this work overestimates the possibility that neutron evaporation inhibits the population of high-energy isomers, because most fission fragments have initial excitation energies sufficiently above the yrast line in the angular-momentum range considered.

\section{J. Emission of Prompt Neutrons and Prompt Gammas}

The de-excitation of the fission fragments after scission, including the acceleration phase, is obtained within the statistical model. Right after scission, the fragments are continuously accelerated in the Coulomb field, starting with their pre-scission kinetic energy. At scission, they are assumed to switch back immediately to their groundstate shape, giving rise to the corresponding increase of intrinsic excitation energy to the initial value $E^{*}$ of the de-excitation process that feeds prompt-neutron and prompt-gamma emission. The neutron and the gamma emission widths are given by

$$
\Gamma_{n}=\frac{(A-1)^{2 / 3} \cdot 0.13 / \mathrm{MeV} \cdot T_{d}^{2}}{\exp \left(S_{n}^{e f f} / T_{d}\right)}
$$

and

$$
\Gamma_{\gamma}=0.62410^{-9} \cdot A^{1.6} \cdot T^{5} / \mathrm{MeV}^{4},
$$

as proposed in Refs. [139] and [66], respectively. $A$ is the mass number and $T$ the nuclear temperature of the fragment, $S_{n}^{e f f}$ is the neutron separation energy, averaged over odd-even fluctuations, $T_{d}$ is the temperature of the daughter nucleus at $E^{*}-S_{n}^{e f f}$.

In addition, the geometrical inverse total neutron cross sections appearing in Eq. (44) were multiplied with the optical-model parameters of Ref. 140]. For calculating the energies of the statistical E1 gammas, the gamma strength of the giant dipole resonance (GDR) following the description proposed in Ref. 141] was applied. The modeling of the level density of the fragments is described in Sec. III B.

Neutron emission during fragment acceleration is considered. It reduces especially the laboratory energies of the first neutrons emitted at short times from highly excited fragments. As already mentioned, it is assumed that both the emission of neutrons and the emission of E1 gammas do not change the angular momentum, which seems to be a good approximation in the relevant angularmomentum range [124]. 
When the yrast line is reached, the angular momentum is carried away by discrete gamma rays, characteristic of the nuclear structure of the specific fission-fragment product. These transitions give rise to a complex peak structure in the low-energy range of the gamma spectrum. In recent work [142, 143], it has been demonstrated that this structure can be well reproduced by taking into account the experimental spectroscopic information of the fission fragments. However, this is not a satisfactory solution for the present purpose, because the GEF code should also be applicable for the fission of any nucleus and for all of the potentially exotic produced fragments for which sufficient information on spectroscopic properties is not necessarily available. Therefore, instead of relying on existing empirical information, an alternative approach is adopted, based on a fully analytical and independent parametrization of the yrast line. It is assumed that, once the yrast line is reached, the angular momentum is carried away by a cascade of E2 gamma transitions, and a special kind of variable-moment-of-inertia (VMI) model [144, 145] has been developed for modeling the angular-momentum dependent energy of that line. The parametrization is particularly, but not only, applicable for the mass range of fission fragments in the low-to-medium angular-momentum range of interest.

The following mathematical formulation is used:

The transition energy $E_{\gamma}$ from angular momentum $J$ to $J-2 \hbar$ is given by

$$
E_{\gamma}=\frac{J_{e f f}\left(J_{e f f}+1 \hbar\right)}{2 I_{e f f}}-\frac{\left(J_{e f f}-2 \hbar\right)\left(J_{e f f}-1 \hbar\right)}{2 I_{e f f}} .
$$

$I_{\text {eff }}$ is given by the following equation

$$
I_{\text {eff }}=0.45 \cdot \frac{1.16^{2} \cdot A^{5 / 3}}{103.8415 \mathrm{MeV} / \hbar^{2}} \cdot U_{\text {shell }}(Z, A) .
$$

The factor 0.45 represents roughly the effect of pairing correlations, and the function $U_{\text {shell }}(Z, A)$ considers the effect of a shell closure on the yrast line at low angular momentum.

Both quantities $J_{\text {eff }}$ and $I_{\text {eff }}$ are parameters of the VMI model. They do not represent the "true" angularmomentum and moment-of-inertia value, respectively. $J_{\text {eff }}$ differs from the "true" $J$ by two functions due to shell and pairing effects, namely

$$
J_{\text {shell }}=f_{\text {shell }}(J)
$$

and

$$
J_{\text {eff }}=f_{\text {pair }}\left(J_{\text {shell }}\right) .
$$

$f_{\text {shell }}$ considers the transition from rotational to vibrational behavior in the vicinity of spherical magic nuclei, and $f_{\text {pair }}$ considers the angular-momentum dependent reduction of the moment of inertia below the critical angular momentum that is set to $12 \hbar$ due to pairing correlations. These are given by the following functions

$$
f_{\text {shell }}(J)=2+(J / \hbar-2) \cdot U_{\text {shell }}^{2}(Z, A)
$$

and

$$
f_{\text {pair }}(J)=0.45 \cdot \frac{J}{\hbar}+0.65 \cdot \max \left(12-\frac{J / \hbar}{12}, 0\right) .
$$

The two-dimensional function $U_{\text {shell }}(Z, A)$ is of the type

$$
\frac{1}{1+W_{n} \cdot\left[N_{\max }-N_{v a l}\right]+W_{z} \cdot\left[Z_{\max }-Z_{\text {val }}\right]},
$$

whereby negative values of the brackets in the denominator are replaced by zero. This function has sharp valleys at shell closures in $N$ and $Z$ and approaches 1 with increasing number of valence neutrons $\left(N_{v a l}\right)$ and valence protons $\left(Z_{\text {val }}\right)$. The shells at proton or neutron number $20,28,50,82$, and 126 are included. In addition, the sub-shell around ${ }^{96} \mathrm{Zr}$ is considered in a similar way. The constants $W_{n}, W_{z}, N_{\max }$, and $Z_{\max }$ are specific to each shell. The parameters are adjusted to reproduce the experimental yrast lines. The full VMI model will be documented in a dedicated publication.

A table of long-lived yrast isomers is provided. If such a long-lived state is met in the E2 cascade, the eventual further emission of delayed gamma radiation is not considered any more.

The achievement of the above-outlined modeling of the yrast line is illustrated below. Figs. 20 to 22 compare the experimental yrast line (black dots) with the new parametrization implemented in GEF (full red squares) and the prediction by the former VMI model 145] (full blue triangles). To have an horizontal scale that is common to all isotopes, with even or odd, integer or not, angular-momentum sequences, the yrast-line energies are shown as a function of so-called state number, with number 0 referring to the band-head ground state. The number of states shown varies from one nucleus to the other, depending on the available experimental information, on one side, and to the highest angular momentum populated in GEF, on the other side. Figs. 20 and 21 show the Sr chain (including an odd-A isotope), and the Sn (upper half) and Sm (lower half) chains, for those isotopes typically populated in low-energy fission of actinides. This permits to appreciate the smooth transition from vibrator-like to rotor-like systems along isotopic chains, and which is exhibited by the progressive change of the shape of the yrast line, from nearly linear (vibrator-like) to parabolic (rotor-like), as a function of angular momentum. The new parametrization developed for GEF is observed to fairly well describe the experimental trend. The level of accuracy reached for the yrast 


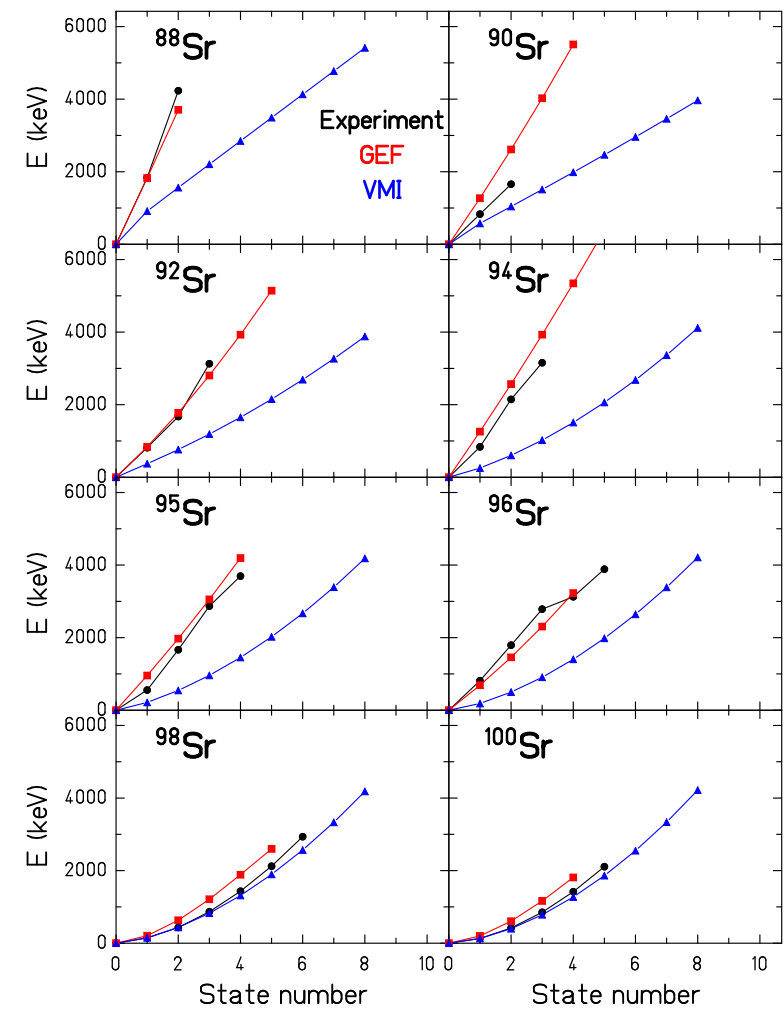

FIG. 20. (Color online) Yrast-line energies with increasing angular momentum presented as a function of state number starting from the ground-state band-head (referred to as state 0 ) for several Sr isotopes as indicated. Experimental energies (black dots) are compared with predictions by the new parametrization developed for GEF (full red squares) and the VMI model of Ref. [145] (full blue triangles).

energies of collective systems is rather good, and much superior to what achieved with the former VMI model in most cases. However, substantial discrepancies appear, when one approaches closed-shell nuclei. The yrast sequence of the latter is very much dependent on the specific single-particle structure, and, therefore, it is difficult to encompass in a global analytical parametrization.

The sample of more fragments typically produced in low-energy fission of actinides displayed in Fig. 22 further corroborates the above observations. There, the $\mathrm{y}$-axis range is limited to $4 \mathrm{MeV}$, in order to facilitate quantitative considerations. In addition, for two fragments $\left({ }^{128} \mathrm{Sn}\right.$ and $\left.{ }^{133} \mathrm{Te}\right)$, which can be populated in an isomeric state, the energy pattern above the isomer is additionally shown with open symbols for the experiment and for the parametrization in GEF. As concluded above, the description of collective patterns is very good. Also, the reproduction of the levels of near-to-closed-shell isotopes can be rather reasonable in some cases, too (see e.g. ${ }^{133} \mathrm{Te}$ and ${ }^{136} \mathrm{Xe}$ ). However, as also observed above, the discrepancy between the GEF level energies and experiment is rather large in some cases. It varies from a few

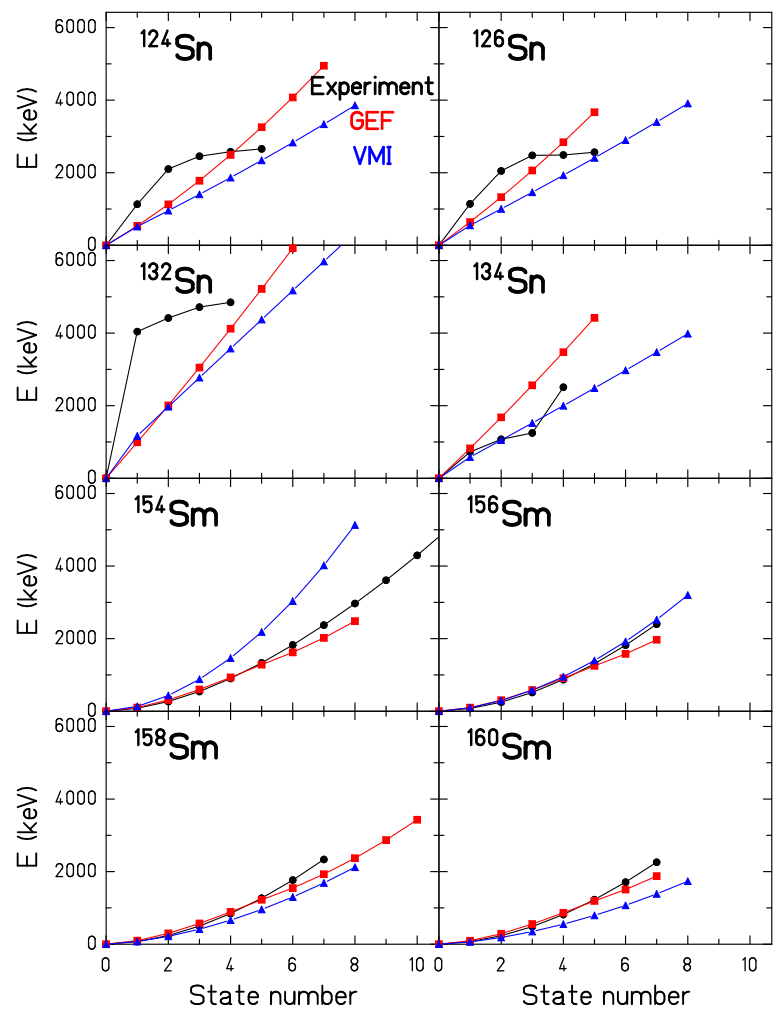

FIG. 21. (Color online) Yrast-line energies with increasing angular momentum presented as a function of state number starting from the ground-state band-head (referred to as state 0 ) for several Sn and Sm isotopes as indicated. Experimental energies (black dots) are compared with predictions by the new parametrization developed for GEF (full red squares) and the VMI model of Ref. [145] (full blue triangles).

tens of $\mathrm{keV}$ up to $1 \mathrm{MeV}$ at low angular momentum, and sometimes even more at higher spin.

\section{PARTICLE-INDUCED FISSION}

For practical reasons, it is desirable that the GEF code also provides a description of the processes that happen in induced fission before the formation of an excited compound nucleus. The technically most important reaction is the interaction of a neutron with a heavy nucleus. But also charged-particle and heavy-ion induced fission is considered, although in a less elaborate way.

\section{A. Neutron-induced Fission}

Neutrons with energies below a few $\mathrm{MeV}$ are absorbed, forming a compound nucleus with an excitation energy equal to the full center-of-mass energy. Interactions at higher energies are characterized by the collision of the incoming neutron with another nucleon and successive 


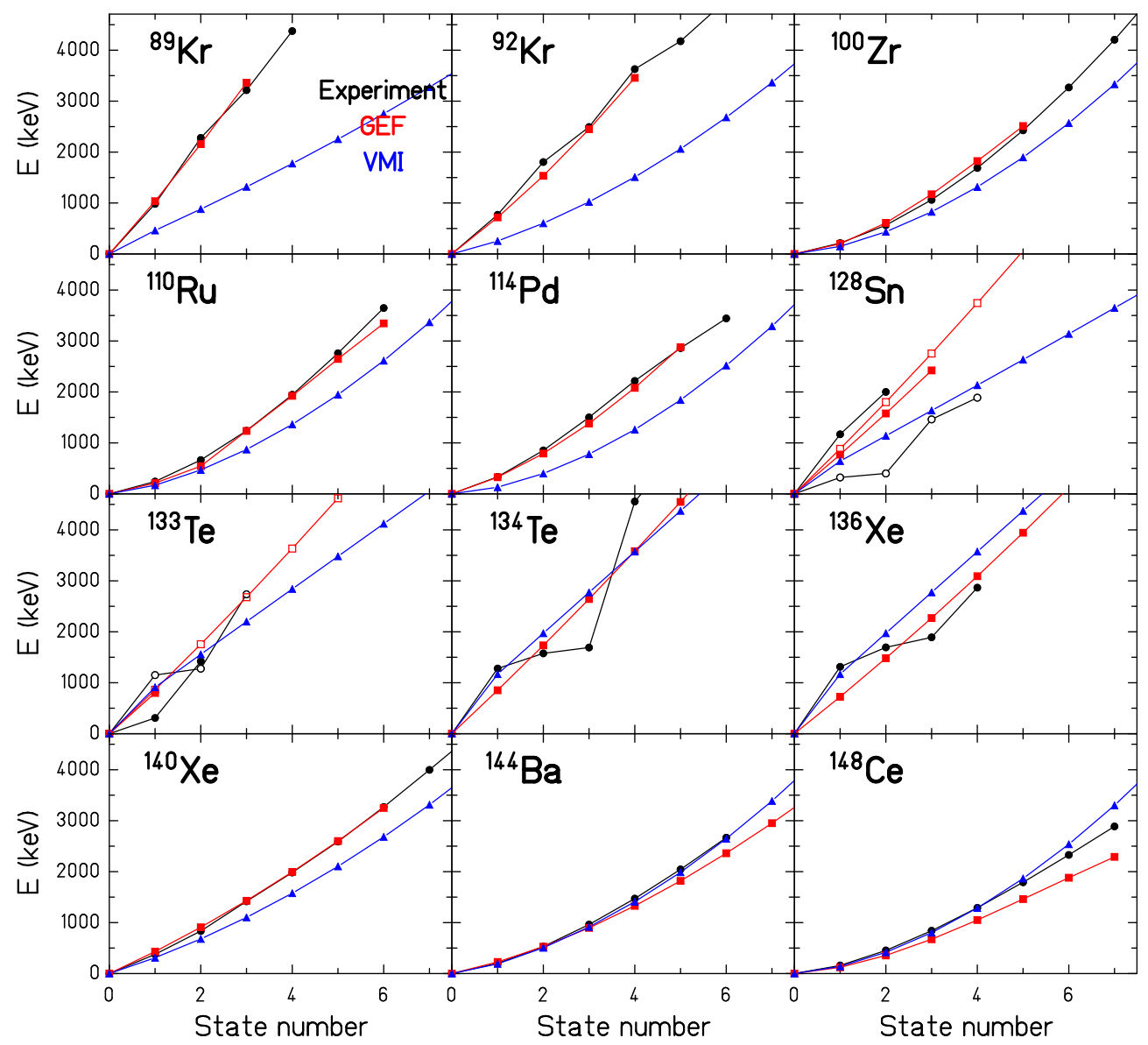

FIG. 22. (Color online) Similar to Fig. 20 for typical fission fragments as indicated. In case of ${ }^{128} \mathrm{Sn}$ and ${ }^{133} \mathrm{Te}$, the evolution of the energies above the isomeric state is shown also (open symbols) for experiment and GEF. Note that the y-axis range is less extended than in Figs. 20 and 21

collisions of the excited nucleons with more and more nucleons of the heavy nucleus. The single-particle configurations develop by more and more complex patterns towards an equilibrated compound nucleus. During this process, some of the excited nucleons may be emitted.

The basic idea for the description of this preequilibrium emission is the evolution of the system towards an increasing number of excited particles and holes (excitons). Particles are emitted from each of these configurations with a characteristic energy spectrum and with essentially equal probability [148]. More sophisticated pre-equilibrium models were developed, e.g. Refs. 149 151, which describe the spectra of the emitted nucleons and eventually also of light clusters with better quality, often with specific empirical adjustments.

Guided by elaborate calculations [152], the probability of pre-equilibrium neutron emission $\left(P_{p e}\right)$ up to $N_{\text {exciton }}=10$ and statistical emission $\left(P_{\text {stat }}\right)$ is assumed to follow a linear dependence as a function of the incident- neutron energy $E_{\text {in }}$

$$
P_{p e} / P_{\text {stat }}=\left(E_{\text {in }} / \mathrm{MeV}-2\right) / 30 \text {. }
$$

The shape of the pre-equilibrium neutron spectrum is essentially given by

$$
d N / d E_{n} \sim v_{n} \cdot\left(E_{\text {in }}-E_{n}\right)^{N_{\text {exciton }} .}
$$

( $v_{n}$ is the velocity of the emitted neutron.) This simple description represents the influence of pre-equilibrium emission on the prompt-neutron spectrum and the fissionfragment yields for a fixed incident-neutron energy rather well.

The angular momentum induced by the incoming neutron, although it is generally rather small, is considered by its effect on the fission barrier, as described in the next subsection. 


\section{B. Charged-particle- and Heavy-ion-induced Fission}

Fission of an excited nucleus emerging from another entrance channel is supported in a less elaborate way. The calculation starts from a compound nucleus with given excitation energy and angular momentum. Preequilibrium processes in light-charged-particle-induced fission and complex features like incomplete fusion, transfer, deep-inelastic reactions and fragmentation in heavyion-induced reactions are not explicitly covered and need to be handled by a dedicated external model. Moreover, quasi-fission, that is a binary reaction without the formation of a compound nucleus, is beyond the scope of the GEF model.

In the fusion of a heavy nucleus and a charged particle, an excited compound nucleus with a characteristic angular-momentum distribution is formed. The angularmomentum range is even broader after the fusion of two heavy nuclei [153]. The main effect of an increased angular momentum is the reduction of the fission barrier 154. If the excitation energy is sufficiently high, this leads to an increased fission probability that modifies the weights of the different fission chances. The increase of the fission-decay width $\Gamma_{f}(J)$ is considered by an additional factor

$$
F_{\text {rot }}=\exp \left(\left(J / J_{\text {lim }}\right)^{2}\right) .
$$

See Sec. IX A 2 for details.

Also the stiffness of the macroscopic potential for massasymmetric shape distortions between saddle and scission depends on the angular momentum and the corresponding distribution of its projections $K$ on the symmetry axis of the fissioning system [154]. This may change the relative yields of the different fission channels. This variation (that is expected to be small for small $K$ values) is not considered. The effect on the width of the mass distribution of the symmetric fission channel is taken into account, see Sec. VIB.

For calculating the observables in charged-particle and heavy-ion-induced fission, the GEF code must be called with the appropriate rms angular momentum defined by the entrance channel.

Another aspect of particle-induced fission, equally valid for neutrons, charged particles and heavy ions, is the influence of the spin distribution of the entrance channel on the angular-momentum distribution of the fission fragments. This aspect is described in Sec. III I

\section{MULTI-CHANCE FISSION}

If the excitation energy of a heavy nucleus is high enough that the excitation energy after particle emission falls near or above the fission barrier of the daughter nucleus, the observed fission events are a mixture from the fission of the mother (first-chance fission) and of the daughter nucleus (second-chance fission). With

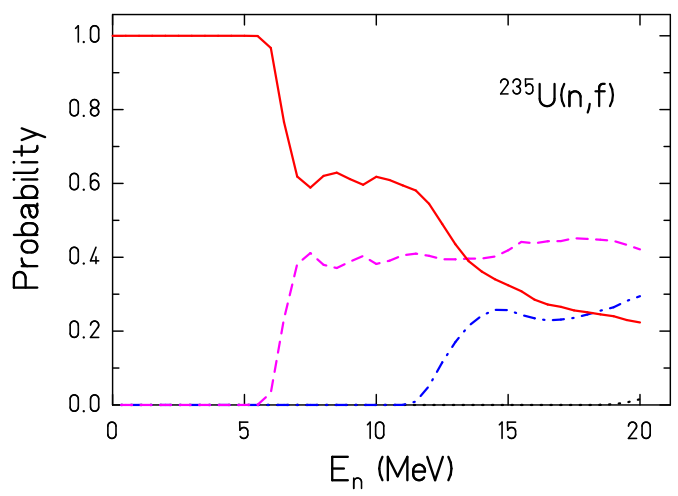

FIG. 23. (Color online) Relative contributions of the different fission chances in the system ${ }^{235} \mathrm{U}(\mathrm{n}, \mathrm{f})$ as a function of the energy of the incident neutron. Full line: first-chance fission, dashed line: second-chance fission, dot-dashed line: third-chance fission.

increasing excitation energy, also the fission of the granddaughter nucleus begins to contribute (third-chance fission), and so on. For the GEF code, it is important to know the relative contributions of the different chances.

Modeling of multi-chance fission requires calculating the competition between particle and gamma decay and fission as a function of excitation energy and angular momentum for determining the relative contributions of the different nuclei and the corresponding excitation-energy distributions at fission. Moreover, the variation of the fis-

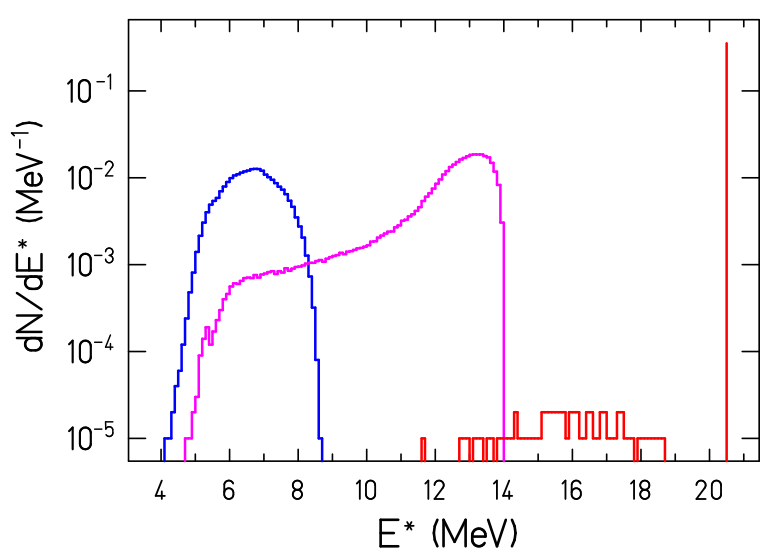

FIG. 24. (Color online) Distribution of excitation energies $E^{*}$ at fission for the system ${ }^{235} \mathrm{U}(\mathrm{n}, \mathrm{f})$ at $E_{n}=14 \mathrm{MeV}$. $E^{*}$ is the excitation energy of the compound nucleus above its ground state, before it passes the fission barrier towards scission. The right-most peak shows events from first-chance fission (fission of ${ }^{236} \mathrm{U}$ ), the middle curve corresponds to second-chance fission (fission of ${ }^{235} \mathrm{U}$ ), and the left curve corresponds to thirdchance fission (fission of ${ }^{234} \mathrm{U}$ ). The broad distribution around $16 \mathrm{MeV}$ corresponds to gamma emission before fission. 
sion characteristics, e.g. the nuclide distributions, needs to be described. Both topics are detailed in other sections of this report. Fig. 23 shows the calculated relative contributions of the different fission chances in the system ${ }^{235} \mathrm{U}(\mathrm{n}, \mathrm{f})$ as a function of the energy of the incident neutron.

The excitation energies at fission corresponding to the different fission chances are shown in Fig. 24 for the system ${ }^{235} \mathrm{U}(\mathrm{n}, \mathrm{f})$ at $E_{n}=14 \mathrm{MeV}$. The events in the broad weak distribution around $16 \mathrm{MeV}$ correspond to pre-fission gamma emission.

Detailed information on the characteristics of multichance fission, in particular at the threshold energies where a new fission chance opens, is very scarce, because there exist no comprehensive high-precision data on nuclide distributions with a fine grid of excitation energies.

\section{PARAMETER VALUES}

According to the concept of the GEF model, a number of parameters were determined from a systematic analysis of empirical data. In the following, the parameters that are relevant for the physics of the model are listed. Some details of predominantly technical nature are not documented in full detail. They may be searched for in the open source of the code [75].

\section{A. Positions of the Fission Channels}

The mean positions of the shell-stabilized heavy fragments of the different fission channels in thermal-neutroninduced fission are given by the following empirical relations:

For the S1 channel

$$
\bar{Z}_{S 1}=51.5+25 \cdot\left(\frac{Z_{C N}^{1.3}}{A_{C N}}-1.5\right),
$$

for the S2 channel

$$
\bar{Z}_{S 2}=53.4+21.67 \cdot\left(\frac{Z_{C N}^{1.3}}{A_{C N}}-1.5\right),
$$

and for the S3 channel

$$
\bar{Z}_{S 3}=58.0+21.67 \cdot\left(\frac{Z_{C N}^{1.3}}{A_{C N}}-1.5\right) .
$$

The exact position of the shell around $Z=42$ in the light fragment that enhances the yield of the S1 channel in fissioning nuclei around $\mathrm{Pu}$ is

$$
\bar{Z}_{\text {light }}=42.15 \text {. }
$$

The shell in the light fragment that enhances the yield of the S3 channel in fissioning nuclei around $\mathrm{Cf}$ has a slightly different position

$$
\bar{Z}_{\text {light }}=39.7 \text {. }
$$

These two values probably refer to the same shell. In addition to the uncertainties of this analysis, the displacement can be explained by the correlation between particle number and deformation for deformed shells as discussed in Sec. VID. The spherical heavy fragment of the S1 channel induces a stronger Coulomb force and thus drives the light fragment to larger deformation and the shell to larger particle number than the strongly deformed heavy fragment of the S3 channel. When this shell in the light fragment meets one of the shells in the heavy fragment, it enhances the yield of the corresponding fission channel, but it is apparently too weak to shift its position. There are indications, however, that this shell has an influence on the deformation of the light fragment at scission, see Sec. VID.

According to the assumption that fragment shells are behind the structural effects that form the fission valleys, this is the same fragment shell, which creates the doublehumped fission-fragment mass distributions for several fissioning systems around $Z=80$ [70 72 .

The positions of the fission channels in fragment mass vary with increasing excitation energy. They are determined by maximizing the level density in the massasymmetry degree of freedom, considering the macroscopic potential in mass-asymmetry and the shell effects.

The positions of the fragment shells are parametrized as a function of nuclear charge $Z$, because the positions of the fission channels are rather constant in $Z$ for the different fissioning systems. This does not necessarily mean that these are proton shells. The sign of the charge polarization rather indicates that the shell behind the most intense asymmetric fission channel S2 is predominantly caused by a neutron shell, as discussed in Sec. IIID

\section{B. Widths of the Fission Channels}

The shape of the potential for mass-asymmetric distortions at the moment of freeze-out of this degree of freedom is the sum of the macroscopic and the microscopic contribution. All these contributions are approximated by parabolas $U=U_{0}+C \cdot\left(Z-Z_{0}\right)^{2}$ in the vicinity of their minima, except for the $\mathrm{S} 2$ fission channel, where the potential has a more complex shape. The values of the stiffness coefficients $C$ are listed in Table IV.

TABLE IV. Stiffness coefficients.

\begin{tabular}{c|c|c|c}
\hline \hline macroscopic & S1 & S3 & $Z \approx 42$ \\
\hline systematics [29] & $0.30 \mathrm{MeV}$ & $0.076 \mathrm{MeV}$ & $0.28 \mathrm{MeV}$ \\
\hline \hline
\end{tabular}

Note: Stiffness coefficients of the different contributions to the potential for mass-asymmetric distortions at freeze out of this degree of freedom. The stiffness of the macroscopic potential depends on the system. It is taken from Ref. [29].

The shell that forms the $\mathrm{S} 2$ channel is parametrized as a rectangular distribution in particle number with a width 
of $\Delta Z=5.6$. The borders are smoothed by a parabolic shape with $C=0.174 \mathrm{MeV}$ at the lower side and with $C=0.095 \mathrm{MeV}$ at the upper side. This is technically performed by convoluting the rectangular distribution with two Gaussian distributions with different width around the two borders of the rectangle. This kind of shape is consistent with the general feature of deformed shells obtained from shell-model calculations [74, 76], which show an extended valley in the 2-dimensional plane of particle number and deformation that starts at a specific particle number at small deformation and extends to a larger particle number at large deformation with a rather constant amount of additional binding over the whole range.

The effect of angular momentum $J$ on the width of the symmetric fission channel is taken into account by assuming that the centrifugal force can approximately be accounted for by an increased fissility [135]. This is done by calculating the stiffness according to the empirical systematics of Rusanov et al. 29] with a modified value of $Z^{2} / A$

$$
\left(Z^{2} / A\right)_{e f f}=Z^{2} / A+\frac{C_{\text {rot }} \cdot(J / \hbar)^{2}}{A^{7 / 3}}
$$

The parameter $C_{\text {rot }}=600$ was adjusted to a systematics of measured mass distributions [29].

\section{Strength of the Fragment Shells}

The strengths of the fragment shells are listed in Table $\mathrm{V}$. These values refer to the configuration, where the population of the fission valleys is determined. This is assumed to be the case a little behind the outer fission barrier. The strength of the shell behind the S1 fission channel varies as a function of neutron excess, because it is created by both the $Z=50$ and the $N=82$ shells. Thus, its strength decreases if the $N / Z$ ratio of the fissioning system deviates from the one of the doubly magic ${ }^{132}$ Sn by

$$
\frac{\delta U_{e f f}}{\mathrm{MeV}}=-1.8 \cdot\left(1-4.5 \cdot\left|82 / 50-N_{C N} / Z_{C N}\right|\right)
$$

The maximum value of the effective shell strength $\delta U_{\text {eff }}$ is the sum of the shell strength $\delta U=-4.6 \mathrm{MeV}$ and the expense $\Delta U_{m a c}=2.8 \mathrm{MeV}$ to be paid to the macroscopic potential due to the unfavorable spherical shape.

TABLE V. Strengths of the fragments shells near the outer fission barrier.

\begin{tabular}{c|c|c}
\hline \hline $\mathrm{S} 2$ & $\mathrm{~S} 3$ & $Z \approx 42$ \\
\hline$-4.0 \mathrm{MeV}$ & $-6.0 \mathrm{MeV}$ & $-1.3 \mathrm{MeV}$ \\
\hline \hline
\end{tabular}

\section{Fragment Deformation}

The shape of the nascent fragments at scission is assumed to be governed by a global feature that is shown by shell-model calculations [74, 76]. Although the results differ in their details, the calculations show regular patterns of valleys and ridges extending from lower particle number and smaller deformation to higher particle number and larger deformation both for neutron and proton shells. Fig. 25 demonstrates this correlation for the neutron subsystem. For example, there are two valleys reaching from $N=60$ and $\beta=0.4$ to $N=70$ and $\beta=$ 0.8 and from $N=78$ and $\beta=0.4$ to $N=92$ and $\beta=$ 0.8. Similar structures appear in the proton subsystem.

This correlation between shell-stabilized shape and size of the nucleus is assumed to govern the deformation of the fission fragments at scission and to explain the saw-tooth behavior of the prompt-neutron yields.

The deformation of the fragments at scission is approximated by a second-order spheroid with a tip distance of $1 \mathrm{fm}$. The deformation parameter $\beta$ of the heavy fragment of the $\mathrm{S} 2$ fission channel is parametrized as a linear function of the atomic number $Z_{\text {heavy }}$

$$
\beta_{\text {heavy }}=0.0275\left(Z_{\text {heavy }}-48.0\right) .
$$

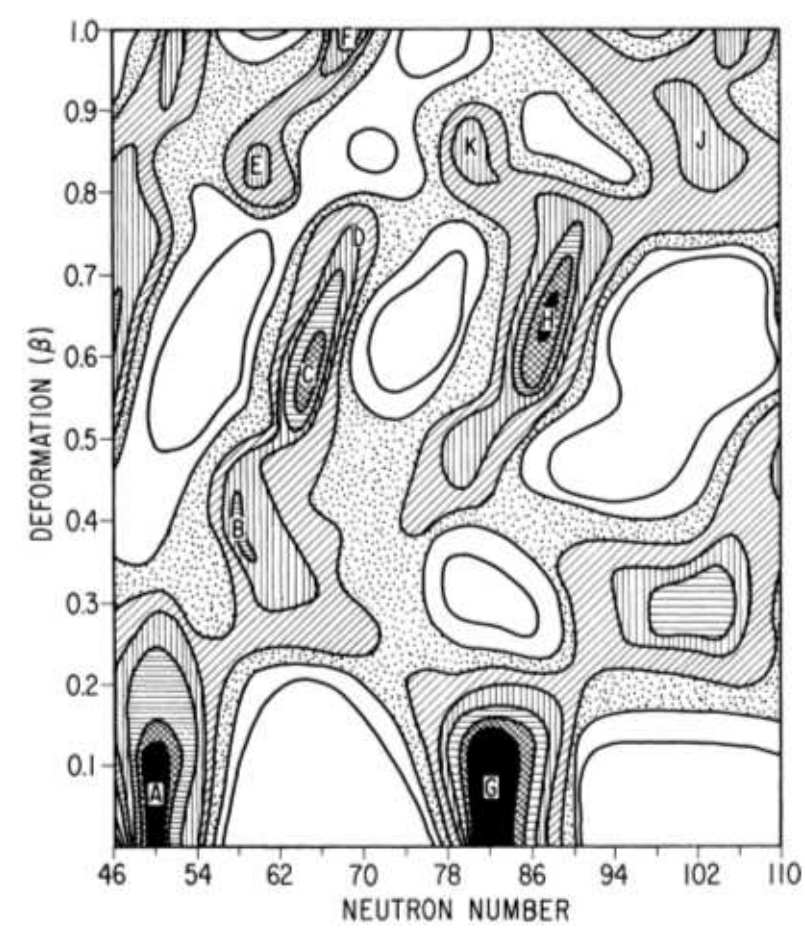

FIG. 25. Neutron-shell corrections calculated as a function of deformation $(\beta)$ and neutron number. The contours are plotted at $1 \mathrm{MeV}$ intervals with the black regions containing all values lower than $-4 \mathrm{MeV}$ and the inner white region containing all values greater than $+2 \mathrm{MeV}$. Pairing and liquid-drop terms are not included. The figure is copied from Ref. [74]. 
The deformation of the light fragment of the S2 fission channels is given by

$$
\beta_{\text {light }}=0.0325\left(Z_{\text {light }}-24.5\right) .
$$

We assume that this is due to a shell, roughly in the region $28<Z<50$. It was not possible to deduce the strength of this shell from the fission observables, but it is certainly weaker than the shells in the heavy fragment, because this shell in the light fragment does not influence the positions of the S1, S2 and S3 fission channels.

Deviating from this behaviour, the nascent heavy fragment of the S1 channel is assumed to be spherical. The deformation of the complementary light fragment is set to

$$
\beta_{\text {light }}=0.0325\left(Z_{\text {light }}-24.5\right)-0.1 \text {. }
$$

in order to reproduce the measured TKE values.

The deformation parameters of the nascent fragments of the super-long (symmetric) fission channel were determined by minimizing the potential energy (binding energies of the fragments plus Coulomb interaction potential) at the scission configuration.

The deformation parameter of the heavy fragment of the S3 fission channel is given by

$$
\beta_{\text {heavy }}=0.0275\left(Z_{\text {heavy }}-48.0\right)+0.2 .
$$

The deformation of the light fragment of the S3 fission channel is

$$
\beta_{\text {light }}=0.0325\left(Z_{\text {light }}-24.5\right)-0.1 .
$$

In all cases, oblate deformation resulting from these formulae was replaced by spherical shape $(\beta=0)$.

\section{E. Charge Polarization}

The charge polarization at scission (related to the deviation of the $N / Z$ ratios of the fragments from the value of the fissioning nucleus) is calculated by minimizing the macroscopic potential energy of the corresponding scission configuration for a given mass division. In order to obtain agreement with experimental data, the mean number of protons in the light (heavy) fragment for a fixed fragment mass is reduced (increased) by 0.32 units, except for the super-long fission channel. This additional charge polarization is attributed to the influence of fragment shells. Because the fragment shells do not depend on the fissioning system, this additional polarization is assumed to be the same for all systems.

\section{F. Energies and Temperatures}

\section{Temperatures}

The width $\sigma$ of the distribution of the coordinate in a quantum oscillator can be expressed by an effective tem- perature $T_{Z}$ that includes the effect of the zero-point motion

$$
\sigma=\sqrt{T_{Z} / C}
$$

with

$$
T_{Z}=\frac{\hbar \omega}{2} \operatorname{coth}\left(\frac{\hbar \omega}{2 T}\right)
$$

The minimum value of the $T_{Z}=\hbar \omega / 2$ is not only specific to the collective coordinate, but, for example for the mass-asymmetry degree of freedom, also to the fission channel considered.

The temperature parameter for the symmetric fission channel that is created by the macroscopic potential is given by the parametrization on the basis of the Fermigas level density of Ref. [29] with a minimum value of $0.72 \mathrm{MeV}$ in the constant-temperature regime. The calculation of the widths of the other fission channels, which are caused by shell effects, is more complex, see above. They are directly parametrized to reproduce the empirical values and their variation with excitation energy. The collective temperature $T$ in the mass-asymmetry degree of freedom is given by

$$
T=0.034 \cdot E_{B}^{*}+0.04 \cdot \Delta E_{p o t} .
$$

$E_{B}^{*}$ is the excitation energy relative to the height of the outer barrier, and $\Delta E_{\text {pot }}$ is the potential-energy gain from the outer barrier to scission.

The values for the quantum oscillator for the chargepolarization degree of freedom are $\hbar \omega=2 \mathrm{MeV}$ and stiffness coefficient $c=3.16 \mathrm{MeV}$ (variation of $Z$ for fixed $A$ ). The width is dominated by the zero-point motion.

\section{Excitation Energy at Scission}

The total excitation energy at scission consists of three contributions $E_{\text {scission }}^{*}=E_{B}^{*}+E_{\text {diss }}+E_{\text {coll }}$ :

1. The initial excitation energy of the fissioning nucleus minus the height of the outer fission barrier

$$
E_{B}^{*}=E_{C N}^{*}-B_{B} .
$$

2. The intrinsic energy acquired through dissipation on the way from the barrier to scission

$$
E_{\text {diss }}=0.35 \cdot \Delta E_{\text {pot }}
$$

with

$$
\Delta E_{\text {pot }} / \mathrm{MeV}=0.08 \cdot\left(Z_{C N}^{2} / A_{C N}^{1 / 3}-1358\right)+11 .
$$

$E_{\text {diss }}$ is roughly $35 \%$ of the potential-energy gain from saddle to scission given in Ref. 94 with a slight modification.

3. The collective energy acquired through coupling between collective degrees of freedom on the way from the outer barrier to scission

$$
E_{\text {coll }}=0.065 \cdot \Delta E_{\text {pot }}^{\text {coll }}
$$


with

$$
\Delta E_{\text {pot }}^{\text {coll }} / \mathrm{MeV}=0.08 \cdot\left(Z_{C N}^{2} / A_{C N}^{1 / 3}-1390\right)+11 .
$$

This is roughly $6.5 \%$ of the potential-energy gain from saddle to scission given in Ref. [94] with a slight modification.

The dissipated energy at scission $E_{\text {diss }}$ is assumed to fluctuate with a standard deviation of $70 \%$, not including negative values. The total intrinsic excitation energy at scission $E_{B}^{*}+E_{d i s s}$ is subject to energy sorting [35]. The collective energy $E_{\text {coll }}$ at scission is shared equally between the fragments.

Note that the excitation-energy-dependent promptneutron multiplicities and total kinetic energies show that due to the lack of suitable transition states below the pairing gap $(2 \cdot \Delta$ for even-even nuclei and $\Delta$ for odd-mass nuclei), fission at excitation energies above the barrier proceeds by an effective barrier that is correspondingly higher. Therefore, $E_{B}^{*}$ is correspondingly reduced.

\section{Deformation Energy}

After scission, the Coulomb repulsion between the fragments and the condition of a quasi-fixed tip-distance are not present any more, and, therefore, the fragments snap to a less deformed shape. In this process, a certain amount of energy is liberated and adds up to the intrinsic energy of the respective fragment. This energy is assumed to be dominated by the macroscopic deformation-energy difference given by the liquid-drop model [155]. Therefore, the contribution due to shell effects is neglected. For the heavy fragments of the S1 fission channel around ${ }^{132} \mathrm{Sn}$ this seems to be a realistic assumption, because these nuclei are nearly spherical at scission and in their ground state. Thus, the shell effect, which is rather strong, does not change. The shell effects of the fragments formed in other fission channels, before and after scission, typically in the order of a few $\mathrm{MeV}$, are small compared to the variation of the macroscopic deformation energy, which reaches up to more than $10 \mathrm{MeV}$.

The energy gain by the fragment deformation at scission is estimated by the difference of the macroscopic deformation energies before and after scisssion according to Ref. [155]. The observed width of the prompt-neutronmultiplicity distribution is well reproduced by assuming that the fragment deformation at scission fluctuates with a standard deviation of $\sigma_{\beta}=0.165$.

\section{Tunneling}

Fission at energies below the outer barrier of a specific fission channel, either in the ground state or at low excitation energies, is characterized by tunneling and a reduced value of $E_{\text {diss }}$. The effective transmission coefficients that determine the populations of the different fission channels are calculated with the Hill-Wheeler formula. The effective $\hbar \omega$ values are expressed by effective temperature parameters $T_{\text {eff }}=\hbar \omega /(2 \pi)$. Slightly different values are used for the different fission channels as listed in Table VI. The slightly larger value for the S1 channel is clearly proven by the data. It is very important for a good reproduction of the data. It may be connected with a smaller effective mass or with a thinner barrier due to the more compact configuration at the scission point for this channel.

The reduced value of $E_{\text {diss }}$ is obtained by the formula

$$
E_{\text {diss }}=0.35 \cdot \Delta E_{\text {pot }}^{\text {intr }}
$$

with

$$
\begin{aligned}
\Delta E_{\text {pot }}^{\text {intr }}=0.08 \cdot( & \left.Z_{C N}^{2} / A_{C N}^{1 / 3}-1358\right) \mathrm{MeV} \\
& +11 \mathrm{MeV}-B_{B}+E_{C N}^{*} .
\end{aligned}
$$

Also the value of $E_{\text {coll }}$ is modified

$$
E_{\text {coll }}=0.065 \cdot \Delta E_{\text {pot }}^{\text {coll }}
$$

with

$$
\begin{array}{r}
\Delta E_{\text {pot }}^{\text {coll }}=0.1 \cdot\left(Z_{C N}^{2} / A_{C N}^{1 / 3}-1390\right) \mathrm{MeV} \\
+11 \mathrm{MeV}-B_{B}+E_{C N}^{*} .
\end{array}
$$

TABLE VI. Effective temperature parameter for tunneling.

\begin{tabular}{c|c|c|c}
\hline \hline SL & S1 & S2 & S3 \\
\hline $0.31 \mathrm{MeV}$ & $0.342 \mathrm{MeV}$ & $0.31 \mathrm{MeV}$ & $0.31 \mathrm{MeV}$ \\
\hline \hline
\end{tabular}

Note: Effective temperatures $T_{e f f}$ for the calculation of the effective transmission coefficients through the outer fission barrier.

\section{G. Concluding Remarks}

Most of the parameters discussed in this section have a physical meaning and, thus, can be rather directly compared with results of microscopic theoretical models. Since these parameters comprise already the knowledge on systematic properties of a large number of systems, this might give a more valuable constraint than the rather complex body of direct experimental information.

Altogether, the number of about 50 parameter values from the simultaneous description of a variety of fission observables for almost 100 systems covering from spontaneous fission to fission at excitation energies of about 100 $\mathrm{MeV}$ is astonishingly modest. One should consider that a comparable number of parameter values was used by Wahl [3] for describing only the fission-fragment yields of only one single system with his empirical description.

The physical background of the description and its simplicity give confidence that the GEF model has a good predictive power for nuclei in the neighborhood of the cases which were used to constrain the model. Exceptions may exist due to very local structural effects. 


\section{COMPUTATIONAL STRUCTURE OF THE GEF CODE}

In the present section, some technical information is given on the computational structure and the handling of the GEF code. For more details, the reader is referred to the supplement to the JEFF Report 24 [2].

\section{A. Flowchart of the GEF Code}

The overall computational structure of the GEF code is illustrated in Fig. 26 that shows a concise flowchart of the code. The flowchart is valid for the case of neutroninduced fission. For the computation of uncertainties and covariances with perturbed model-parameter values that is described in Sec. VIII, the complete sequence of calcu-

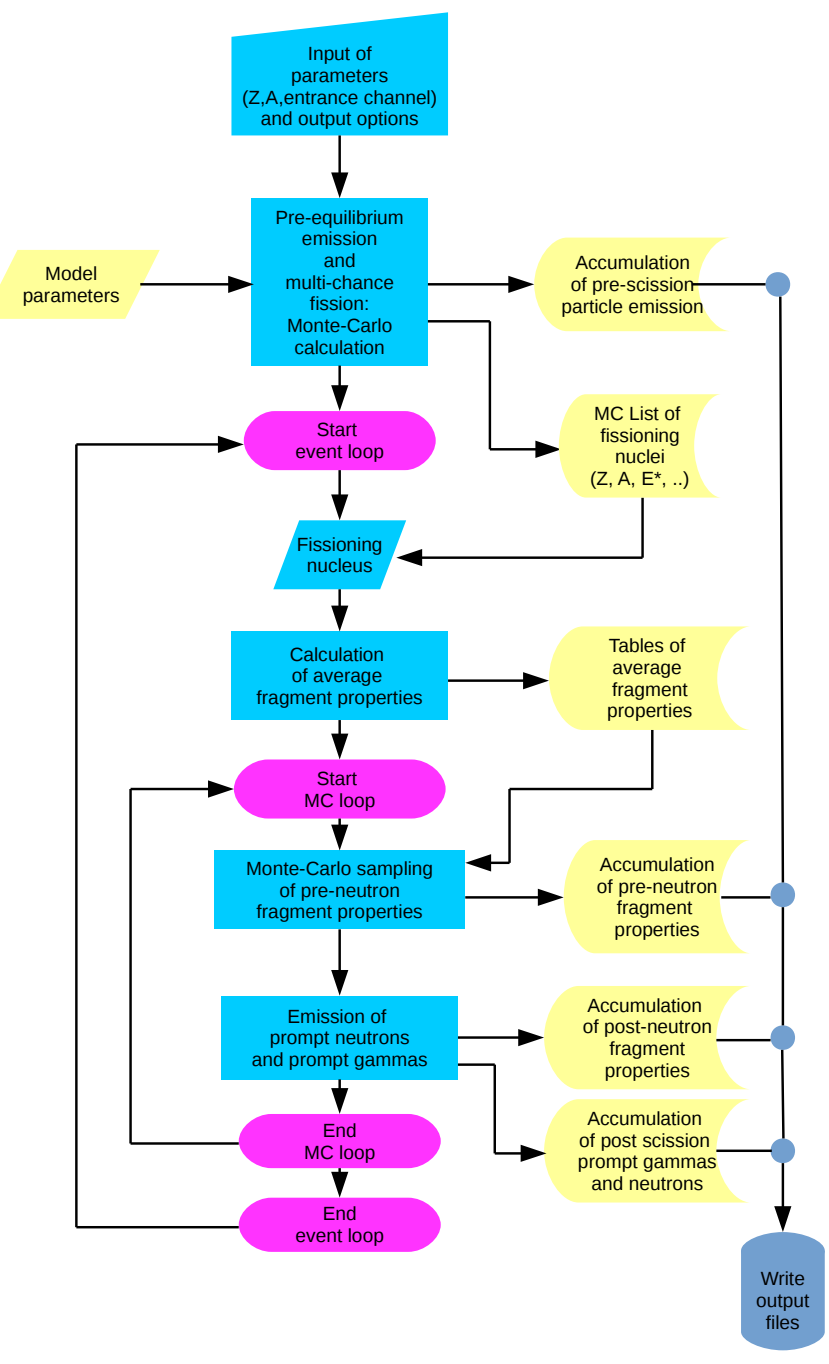

FIG. 26. (Color online) Flowchart of the GEF code. lations is repeated with different parameter values, and the results are stored and further processed.

One can grossly distinguish three computational sections, concerning pre-fission processes, average fragment properties and event-wise calculation of individual fission events.

\section{Pre-fission Processes}

This section is bypassed if the excitation energy of the system does not exceed the particle-emission threshold. It is organized as an independent Monte-Carlo routine. It considers the decay of the system by particle emission, by both pre-equilibrium processes and emission from the compound nucleus. Emission of neutrons and protons is considered. The result of this section is a list of excited nuclei going to fission. They are characterized by mass number $A$, atomic number $Z$, excitation energy $E^{*}$, and angular momentum. Moreover, this list comprises the properties of the emitted particles.

When the number of required events is reached, the events are ordered according to $A, Z$ and $E^{*}$, because this makes the following calculations more efficient. If the GEF code is to be used as an event generator, it is important to know this feature, because the sequence of events in the list-mode output file follows this ordering. For restoring a true random sequence of events, the fission events must be taken in a random sequence from the listmode output file.

\section{Average Fission-fragment Properties}

The input of this computing section is the compound nucleus going to fission, characterized by mass number $A$, atomic number $Z$, excitation energy $E^{*}$, and rms angular momentum $l_{r m s}$. The following key quantities are calculated that define the distributions for the Monte-Carlo sampling of the individual fragment properties that is performed in the next section of the code:

- Central $Z$ values of fragment shells.

- $Z$-dependent mean fragment deformation per mode.

- Curvature of macroscopic potential energy.

- Mean excitation energy above lowest saddle per mode.

- Collective temperatures per mode.

- Central positions in $Z$ of fission modes.

- Mass-dependent mean charge polarization per mode.

- Energy- and mass-dependent mean fragment deformation per mode.

- Mean $Z$ value as a function of $A$ per mode.

- Yields of fission modes.

- Widths of fission modes.

- Mean intrinsic excitation energies at scission.

- Mean intrinsic temperatures at scission.

- Local odd-even effect in $Z$ and $N$ at scission. 
- Mean intrinsic temperatures of final fragments.

- RMS fragment angular momentum.

\section{Monte-Carlo Sampling of Fission Events}

By use of the distributions of fragment properties provided by the previous computing section, the properties of individual fission events are calculated by Monte-Carlo sampling. All possible correlations are taken into account by respecting all possible conservation laws. The result is accumulated and stored in a number of arrays, and eventually all calculated observables are printed event-wise in a list-mode file. The statistical uncertainty of the resulting distributions depends on the number of processed events. In detail, the following properties are determined:

- Fission mode*.

- Mass division*.

- $Z$ division, including odd-even effect*

- Q value for each fragment split.

- Fragment deformation energy at scission*.

- Fragment intrinsic excitation energy at scission*.

- Collective excitation energy at scission*.

- Fragment angular momentum*.

- Pre-neutron total kinetic energy.

- Emission of prompt neutrons and gammas*.

The quantities marked by an asterisk are sampled at random.

\section{B. Subroutines}

The function getyield returns the unnormalized yield of a fission channel.

Input:

- Excitation energy relative to the outer-barrier height.

- Temperature above the barrier (constant-temperature regime).

- Effective temperature below the barrier (for tunneling).

The function masscurv returns the curvature of the macroscopic potential for mass-asymmetric distortions according to the systematics of Rusanov et al.

Input:

$-Z$ of fissioning nucleus

- $A$ of fissioning nucleus

The function d_e_saddle_scission returns the potentialenergy gain from fission barrier to scission according to Asghar and Hasse.

Input:

$-Z^{2} / A^{1 / 3}$ of fissioning nucleus

The function t_egidy returns the temperature parameter of the constant-temperature nuclear-level-density formula of von Egidy et al.

Input:

- Nuclear mass number $A$
- Shell effect

The function t_rusanov returns the temperature of the Fermi-gas nuclear-level-density formula of Rusanov et al.

Input:

- Excitation energy

- Nuclear mass number $A$

The function lymass returns the nuclear mass according to the liquid drop model of Myers and Swiatecki. Input:

- Atomic number $Z$

- Nuclear mass number $A$

- Deformation parameter $\beta$

The function lypair returns the pairing-fluctuation energy according to the liquid-drop model of Myers and Swiatecki.

Input:

- Atomic number $Z$

- Nuclear mass number $A$

The function fedefolys returns the nuclear deformation energy according to the liquid-drop model of Myers and Swiatecki.

Input:

- Atomic number $Z$

- Nuclear mass number $A$

- Deformation parameter $\beta$

The function ldmass returns the macroscopic nuclear mass according to the Thomas-Fermi model of Myers and Swiatecki.

Input:

- Atomic number $Z$

- Nuclear mass number $A$

- Deformation parameter $\beta$

The function ame2012 returns the nuclear mass from the 2012 mass evaluation.

Input:

- Atomic number $Z$

- Nuclear mass number $A$

The function $u \_s h e l l$ returns the ground-state shell effect from the Strutinsky-type model calculation of Möller et al.

Input:

- Atomic number $Z$

- Nuclear mass number $A$

The function $u \_s h e l l \_$exp returns the ground-state shell effect from the difference of empirical mass and Thomas-Fermi mass without even-odd fluctuations.

Input:

- Atomic number $Z$

- Nuclear mass number $A$ 
The function u_shell_e0_exp returns the difference of the empirical mass and the Thomas-Fermi mass. It includes shell effect and pairing fluctuation.

Input:

- Atomic number $Z$

- Nuclear mass number $A$

The function u_mass returns the Thomas-Fermi macroscopic mass plus the ground-state shell correction of Möller et al.

Input:

- Atomic number $Z$

- Nuclear mass number $A$

The function ecoul returns the Coulomb repulsion between two nuclei in the tip-tip configuration.

Input:

- $Z_{1}, A_{1}, \beta_{1}, Z_{2}, A_{2}, \beta_{2}$, tip distance $d$

The function beta_light returns the mean deformation of the light fragment of the $\mathrm{S} 2$ fission channel.

Input:

- Atomic number $Z$ of light fragment

The function beta_heavy returns the mean deformation of the heavy fragment of the S2 fission channel.

Input:

- Atomic number $Z$ of heavy fragment

The function $z_{-} e q u i$ determines the charge polarization and returns $Z_{1}$ in a configuration of two deformed nuclei $\left(Z_{1}, A_{1}, \beta_{1}, Z_{2}, A_{2}, \beta_{2}\right)$ in tip-tip-configuration with a tip distance $d$ by minimizing the total potential energy.

Input:

$-Z_{C} N, A_{1}, A_{2}, \beta_{1}$, beta $2, d$

The subroutine beta_opt_light determines the optimum deformation $\beta_{2}$ of the light fragment when the deformation $\beta_{1}$ of the heavy fragment is imposed in a tip-tip configuration.

Input:

- $A_{1}, A_{2}, Z_{1}, Z_{2}, d, \beta_{2}$

The subroutine beta_equi determines the optimum deformation parameters of two deformed nuclei in a tip-tip configuration.

Input:

- $A_{1}, A_{2}, Z_{1}, Z_{2}$, tip distance $d$

The subroutine eva is a simple evaporation code, used for the fragment de-excitation cascade. It considers neutron evaporation and statistical E1 gamma emission. The subroutine eva returns for neutron evaporation the times after scission and the kinetic energies of the neutrons, for gamma emission the energies of the photons, and the composition $(Z$ and $A$ ) and the excitation energy of the residual nucleus.

The function $u_{-}$accel returns the velocity of the fragment 1 at time $T_{n}$ after scission in units of $\sqrt{(E / \mathrm{MeV}) / A}$.

Input:

- $A_{1}, Z_{1}, A_{2}, Z_{2}$, pre-scission $T K E$

Random generator of gamma energy for gamma emission below the neutron separation energy.

Input:

- Atomic number $Z$

- Nuclear mass number $A$

- Initial excitation energy $E^{*}$

Random generator of gamma energy for gamma emission above the neutron separation energy.

Input:

- Atomic number $Z$

- Nuclear mass number $A$

- Initial excitation energy $E^{*}$

The function u_ired returns the rigid-body nuclear moment of inertia, including the influence of pairing correlations.

Input:

- Atomic number $Z$

- Nuclear mass number $A$

The function u_iredff returns the nuclear moment of inertia with the influence of pairing correlations and nuclear-structure effects. It is calculated as the product of u_ired and u_i_shell.

Input:

- Atomic number $Z$

- Nuclear mass number $A$

The function $u_{-} i_{-}$shell returns a factor that represents the influence of nuclear structure on the nuclear moment of inertia. It is a kind of a modified variablemoment-of-inertia (VMI) model that describes the yrast line of the fission fragments.

Input:

- Atomic number $Z$

- Nuclear mass number $A$

The function $u_{-} a l e v_{-} l d$ returns the macroscopic leveldensity parameter of the Fermi-gas formula according to Ignatyuk.

Input:

- Atomic number $Z$

- Nuclear mass number $A$

The function $u_{-} t e m p$ returns the nuclear temperature parameter from the modified composite level-density formula of Schmidt and Jurado with the influence of shells and pairing correlations (optional).

Input:

- Atomic number $Z$ 
- Nuclear mass number $A$

- Excitation energy $E^{*}$

The function gggtot returns the probability to emit a gamma of energy $E_{\gamma}$ in competition with neutron emission.

Input:

- Atomic number $Z$ of emitting nucleus

- Mass number $A$ of emitting nucleus

- Excitation energy $E^{*}$ of the emitting nucleus

- Energy $E_{\gamma}$ of the emitted gamma

The function bftf returns the height of the fission barrier with shell effects and pairing correlations considered (optional).

Input:

- Atomic number $Z$

- Nuclear mass number $A$

The function bfta returns the height of the inner fission barrier with shell effects and pairing correlations considered (optional).

Input:

- Atomic number $Z$

- Nuclear mass number $A$

The function bftfb returns the height of the outer fission barrier with shell effects and pairing correlations considered (optional).

Input:

- Atomic number $Z$

- Nuclear mass number $A$

\section{Input and Output}

\section{Input}

Required input of GEF:

- $Z, A$ and rms angular momentum of the fissioning nucleus or target.

- Excitation mode and excitation energy.

The user is guided through additional input options by the input dialogue (on LINUX) or by the GUI (on WINDOWS ${ }^{\#}$.

\#) WINDOWS is either a registered trademark or a trademark of Microsoft Corporation in the United States of America and/or other countries.

\section{Output}

a. Tables The following quantities are available on output of GEF:

- Contributions of fission chances.
- Relative yields of fission channels.

- Element-yield distribution*).

- Isotonic-yield distribution (pre- and post-neutron).

- Isobaric-yield distribution*).

- Mass-chain yields (pre- and post-neutron)*).

- Fragment kinetic energies.

- Fragment angular-momentum distributions (for every nuclide).

- Relative independent isomeric yields.

- Prompt-neutron spectrum.

- Neutron-multiplicity distribution.

- Energies and directions of prompt neutrons (preand post-scission).

- Prompt-gamma spectrum.

- Gamma-multiplicity distribution.

*) Including uncertainties and covariances.

Many more quantities are internally calculated and may be listed.

b. List-mode Output The optional list-mode output comprises many properties of the fission fragments and the prompt neutrons and gammas on an event-by-event basis. A short sample of the thermal-neutron-induced fission of ${ }^{235} \mathrm{U}$ is listed below. It demonstrates the large coverage of fission quantities that can all be obtained in correlation with each other, as for example angular correlations of the emitted neutrons [156]. Moreover, complex experimental cuts can easily be applied.

\footnotetext{
- Sample:

* Z1 Z2 A1pre A2pre A1post A2post I1pre I2pre I1gs I2gs Eexc1 Eexc2 n1 n2 TKEpre TKEpost * Z1: Atomic number of first fragment

* Z2: Atomic number of second fragment

* A1pre: Pre-neutron mass number of first fragment

* A2pre: Pre-neutron mass number of second fragment

* A2post: Post-neutron mass number of second fragment

* I1pre: Spin of first fragment after scission

* I2pre: Spin of second fragment after scission

* I1gs: Ground-state spin of first fragment

* I2gs: Ground-state spin of second fragment

* Eexc1: Excitation energy of first fragment [MeV]

* Eexc2: Excitation energy of second fragment [MeV]

* n1: Prompt neutrons emitted from first fragment

* n2: Primpt neutrons emitted from second fragmen

* TKEpre: Pre-neutron total kinetic energy [MeV]

* TKEpost: Post-neutron total kinetic energy [MeV]

In separate lines: Prompt post-scission neutrons (including acceleration phase)

* O E1, cos(theta1), phi1, E2, cos(theta2), phi2, E3, cos(theta3, phi3, ...

Energies [MeV] in lab. frame and angles vs. direction of light fragment

of all post-scission neutrons

* 1 E11, E21, E31, ...: Energies [MeV] of neutrons emitted from light fragment . in frame of light fragment

* 2 E1h, E2h, E3h, ...: Energies [MeV] of neutrons emitted from heavy fragment .

The in frate lines: Prompt post-scission gamm

* 3 E11, E21, E31, ...: Energies [MeV] of gammas emitted from light fragment ..

in competition with neutron

4 ETl, E21, E3, .... Energies [MeV] of statistical gammas emitted from..

light fragment after neutron emission

* 5 E11, E21, E31, ...: Energies $[\mathrm{MeV}]$ of collective gammas emitted from .

* light fragmen

...: Energies

in competition with neutrons

heavy fragment after neutron emissio heavy fragment
} 


\section{UNCERTAINTIES AND COVARIANCES}

Experimental data or results of a model calculation are not expected to be precise. Generally they are subject to an uncertainty margin. In both cases, it is important to provide a realistic estimation of the uncertainty. However, for estimating the uncertainty of an integral derived quantity that depends on many values, e.g. a whole distribution, the knowledge of the uncertainty of individual data points is not sufficient.

Often, the variations of different data points are correlated by a contribution from a common source. A simple case for a common error source for all measured data concerned is a global normalization. The uncertainty of the normalization acts on all data points in a fully correlated way. In the case of an efficiency curve that is known to be smooth, the correlation will decrease with the distance between the points of interest. Also, in the calculated distribution of some observables there exist correlations between different values, but they have a different origin. If a specific property of the system is changed, this has an influence on the values of many observables. For example, a decreased energy dissipation lowers the intrinsic excitation energy at scission and raises the even-odd effect of the element yields, leading to higher yields for even- $Z$ and lower yields for odd- $Z$ elements. The fissionfragment yields in the same fission channel are connected by a positive correlation.

The GEF code provides uncertainties and covariance data for the element yields, the mass yields and the nuclide yields (depending on $Z$ and $A$ ), the latter ones before and after emission of prompt neutrons. Covariances between any other pair of observables can be determined by analyzing the list-mode output of the GEF code. The covariance between two observables $x$ and $y$ is determined by performing a large number $N$ of calculations with different sets of parameters $\vec{p}_{i}=\left(p_{1}, p_{2}, \cdots, p_{n}\right)_{i}$. The index $i$ denotes a specific set of parameters. In each set of parameters $\vec{p}_{i}$ the values of the different parameters are chosen randomly from a normal distribution with a central value given by the nominal parameter value of the model and a standard deviation defined by the uncertainty range of this parameter. The uncertainty range of a specific parameter of the GEF model was determined by investigating, how much the parameter value can deviate from the nominal value, until the agreement with the body of empirical data deteriorates substantially. This analysis was done with some caution, considering that the comprehensive comparison of the data with the GEF results gave occasion to distrust some of the experimental or evaluated data. The determined uncertainties are listed in Table VII. Each parameter is varied independently from the others. The covariance between the two observables $x$ and $y$ is defined by

$$
\operatorname{Cov}(x, y)=\frac{\sum_{i=1}^{N}\left(x_{i}-\bar{x}\right)\left(y_{i}-\bar{y}\right)}{N-1}
$$

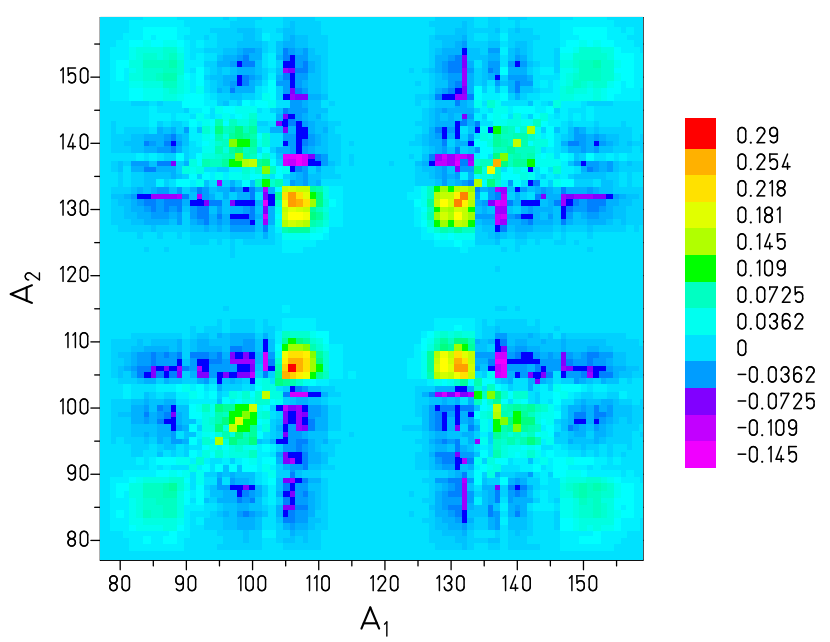

FIG. 27. (Color online) Covariance matrix of the fissionfragment mass yields after prompt-neutron emission from the thermal-neutron-induced fission of ${ }^{239} \mathrm{Pu}$.

$x_{i}$ and $y_{i}$ are the values of the observables $x$ and $y$ from the individual calculations with perturbed parameters, $\bar{x}$ is the mean value of the observable $x$ and $\bar{y}$ is the mean value of the observable $y$ of all $N$ calculations. The values of the covariances of a set of observables, e.g. the yields of the fission-fragment $Z$ distribution form a matrix.

Fig. 27 shows the covariance matrix of the mass yields after prompt-neutron emission for the system ${ }^{239} \mathrm{Pu}\left(\mathrm{n}_{\mathrm{th}}, \mathrm{f}\right)$. The values on the diagonal from the lowerleft corner to the upper-right corner show the largest positive values. They are identical with the variances of the mass yields. Also, the values from the upper-left corner to the lower-right corner are positive. These are the covariances between complementary masses. Due to emission of prompt neutrons, the largest covariance values are slightly smaller and a bit shifted from the diagonal to the left-lower side. The values of the covariances between the yields of masses from different fission channels are negative. This is a consequence of the normalization of the yields to $200 \%$. The post-neutron mass yields of ${ }^{239} \mathrm{Pu}\left(\mathrm{n}_{\mathrm{th}}, \mathrm{f}\right)$ including the error bars determined with perturbed-parameter calculations from the GEF code are compared with the evaluated data from ENDF/B-VII in Fig. 28. The estimated uncertainties of the evaluated data can be seen in Figs. 40 and 41.

Assuming that the model is realistic, the covariances between different observables of a model calculation provide valuable information on the inherent relations between the different observables imparted by the physics of the fission process. This information can be used as a tool to verify the consistency of experimental data and to make evaluations more efficient. 
TABLE VII. Standard deviations of perturbed parameter values.

\begin{tabular}{|c|c|c|}
\hline Quantity & $\bar{\sigma}$ & unit \\
\hline Position of the shell for S1 channel & 0.1 & $Z$ units \\
\hline Position of the shell for S2 channel & 0.1 & $Z$ units \\
\hline Rectangular contribution to the width of $\mathrm{S} 2$ channel & 0.05 & Mass units \\
\hline Position of the shell for S3 channel & 0.1 & $Z$ units \\
\hline Position of the shell at $Z \approx 42$ & 0.1 & $Z$ units \\
\hline Shell effect at mass symmetry & 0.1 & $\mathrm{MeV}$ \\
\hline Shell effect for S1 channel & 0.1 & $\mathrm{MeV}$ \\
\hline Shell effect for $\mathrm{S} 2$ channel & 0.1 & $\mathrm{MeV}$ \\
\hline Shell effect for S3 channel & 0.2 & $\mathrm{MeV}$ \\
\hline Shell at $Z \approx 42$ & 0.05 & $\mathrm{MeV}$ \\
\hline Curvature of shell for S1 channel & 5 & $\%$ \\
\hline Curvature of shell for $\mathrm{S} 2$ channel & 5 & $\%$ \\
\hline Curvature of shell for S3 channel & 5 & $\%$ \\
\hline Curvature of shell at $Z \approx 42$ & 5 & $\%$ \\
\hline$(\hbar \omega)_{e f f}$ for tunneling of S1 channel & 3 & $\%$ \\
\hline$(\hbar \omega)_{e f f}$ for tunneling of S2 channel & 3 & $\%$ \\
\hline$(\hbar \omega)_{e f f}$ for tunneling of S3 channel & 3 & $\%$ \\
\hline$(\hbar \omega)_{e f f}$ for tunneling of channel at $Z \approx 42$ & 3 & $\%$ \\
\hline Weakening of the $\mathrm{S} 1$ shell with $82 / 50-N_{C N} / Z_{C N}$ & 20 & $\%$ \\
\hline Width of the fragment distribution in $N / Z$ & 10 & $\%$ \\
\hline Charge polarization $(\bar{Z}$ for fixed $A)$ & 0.1 & $Z$ units \\
\hline
\end{tabular}

Note: Standard deviations of the parameter values used for determining the uncertainties and the covariances of the GEF results.

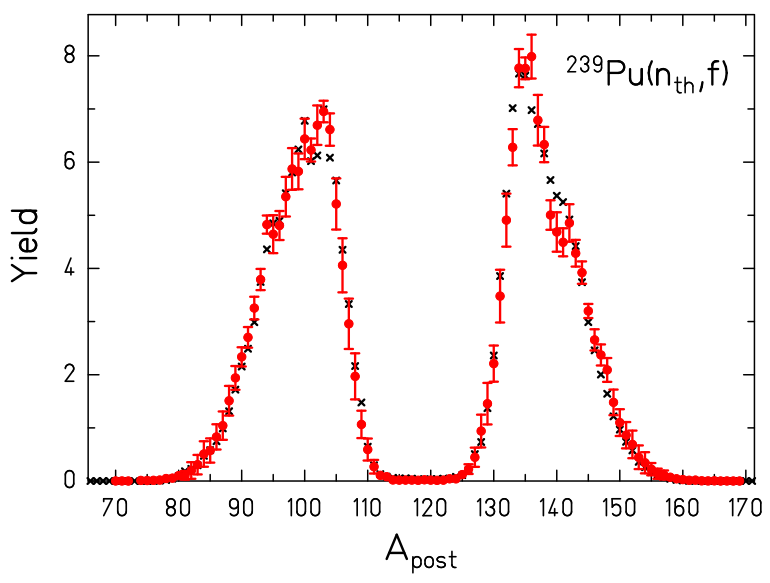

FIG. 28. (Color online) Uncertainties of mass yields from perturbed-parameter calculations. Mass yields after promptneutron emission from the thermal-neutron-induced fission of ${ }^{239} \mathrm{Pu}$. The GEF result (red full points) with error bars is compared with the evaluated data from ENDF/B VII (black crosses).

\section{ASSESSMENT}

\section{A. Fission Probability}

\section{Introduction}

The description of fission observables above the threshold of multi-chance fission requires the knowledge of the competition between fission, neutron emission and gamma decay as a function of excitation energy and angular momentum of the compound nucleus, because they determine the relative weights of the different chances. Entrance-channel-specific pre-compound processes must eventually be considered in addition. They are not included in the present study. Since the GEF code aims at modeling the fission process in a global way without being locally adjusted to experimental data of specific systems, global descriptions of the relevant decay widths are required. This ensures that the GEF code can predict fission observables for systems for which no experimental data are available. However, this also means that specific nuclear-structure effects can only be considered in a global approximate way.

\section{Formulation of the Fission Probability}

The fission probability is calculated as

$$
P_{f}=\Gamma_{f} /\left(\Gamma_{f}+\Gamma_{n}+\Gamma_{\gamma}\right)
$$


The gamma-decay width is calculated by the global formula

$$
\Gamma_{\gamma}=0.62410^{-9} \cdot A_{C N}^{1.6} \cdot T_{i}^{5} / \mathrm{MeV}^{4}
$$

proposed by Ignatyuk [66]. $A_{C N}$ is the mass number and $T_{i}$ is the temperature of the compound nucleus with energy $E_{i}$. (This formula is also applied as Eq. (45) to the gamma emission from the fragments.)

The neutron-decay width is calculated by the global formula

$$
\Gamma_{n}=\frac{0.13}{\mathrm{MeV}} \cdot\left(A_{C N}-1\right)^{2 / 3} \cdot \frac{T_{n}^{2}}{\exp \left(<S_{n}>/ T_{n}\right)}
$$

proposed in Ref. [139], which is valid for an exponential neutron-energy spectrum. $S_{n}$ is the neutron separation energy, $T_{n}$ is the maximum temperature of the daughter nucleus at the energy $E_{i}-<S_{n}>$. This expression is multiplied by

$$
1-\exp \left(-\left(E_{i}-<S_{n}>\right) /\left(1.6 \cdot T_{n}\right)\right)
$$

in order to approximately adapt to the Maxwellian shape of the neutron-energy spectrum. The use of $\left\langle S_{n}\right\rangle=$ $S_{2 n} / 2$ is another way to consider the shift of the level density by $\Delta$ and $2 \Delta$ in odd-mass and even-even nuclei, respectively, with respect to odd-odd nuclei. $\Gamma_{n}$ is set to zero at energies below the neutron separation energy $S_{n}$.

The calculation of the fission-decay width is based on the following equations proposed in Ref. [157] with a few extensions

$$
\Gamma_{f}=F_{\text {rot }} \cdot T_{f} /\left(G \cdot \exp \left(B_{\max } / T_{f}\right)\right) .
$$

$B_{\max }$ is the maximum value of the inner fission barrier $B_{A}$ and the outer fission barrier $B_{B}, T_{f}$ is the temperature of the compound nucleus at the barrier $B_{\max }$. $F_{\text {rot }}$ considers the influence of the root-mean square value $J_{r m s}$ of the angular-momentum distribution of the compound nucleus which depends on nuclear temperature and fissility [135]. It is given by

$$
F_{\text {rot }}=\exp \left(\left(J_{\text {rms }} / J_{\text {lim }}\right)^{2}\right)
$$

with

$$
J_{l i m}=7.93 \hbar \cdot \sqrt{\frac{T_{f}}{T_{c t} \cdot(1-x)}} .
$$

$T_{c t}$ is the nuclear temperature in the constanttemperature regime, and $x$ is the fissility parameter.

$$
\begin{array}{r}
G=G_{A} \cdot \exp \left(\left(B_{A}-B_{\max }\right) / T_{f}\right)+ \\
G_{B} \cdot \exp \left(\left(B_{B}-B_{\max }\right) / T_{f}\right)
\end{array}
$$

whereby $G_{A}$ and $G_{B}$ consider the collective enhancement of the level densities on top of the inner barrier (assuming triaxial shapes) and the outer barrier (assuming massasymmetric shapes) and of tunneling through the corresponding barrier

$$
G_{A}=F_{A} \cdot 0.14 / \sqrt{\pi / 2}
$$

$$
F_{A}=1 /\left(1+\exp \left(-\left(E-B_{A}\right) / T_{\text {equi }}\right)\right.
$$

$$
G_{B}=F_{B} / 2
$$

and

$$
F_{B}=1 /\left(1+\exp \left(-\left(E-B_{B}\right) / T_{\text {equi }}\right)\right.
$$

$T_{\text {equi }}$ is related to the values of $\hbar \omega_{A}$ and $\hbar \omega_{B}$ at the inner and outer barriers by $T_{\text {equi }}=\hbar \omega / 2 \pi$, assuming $\hbar \omega_{A}=$ $\hbar \omega_{B}=0.9 \mathrm{MeV}$.

In order to account for the low level density above $B_{\max }$ at energies below the pairing gap $2 \Delta$ in even-even nuclei, the value of $\Gamma_{f}$ was multiplied at energies in the vicinity of the barrier $B_{\max }$ by a reduction factor that was deduced from the average behavior of measured fission probabilities. The function is shown in Fig. 29.

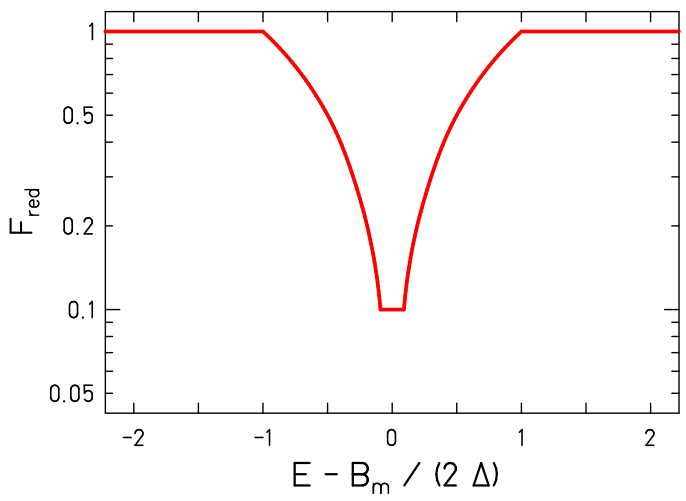

FIG. 29. (Color online) Adapted reduction of the fission-decay width around the fission barrier for even-even nuclei.

The collective-enhancement factors at the inner and outer barrier with respect to the daughter nucleus after neutron decay that is assumed to have a quadrupole shape (the inverse of $0.14 / \sqrt{\pi / 2}$ and 0.5 , respectively) are assumed to fade out at higher energies, where the shape of the fissioning nucleus at scission becomes mass symmetric. They are multiplied by the attenuation factor

$$
F_{a t t}=\frac{\exp \left(0.05\left(E-B_{A}\right)\right)}{1 / G_{A}+\exp \left(0.05\left(E-B_{A}\right)\right)}
$$

for the inner barrier and an analogous factor for the outer barrier.

The temperature values are determined as the inverse logarithmic derivative of the nuclear level density with respect to excitation energy. The nuclear level density both in the ground-state minimum and at the fission barrier are modeled as described in section IIIB. Because the level density in the low-energy range is described by the 
constant-temperature formula, a constant spin-cutoff parameter is used.

The fission barriers are modeled on the basis of the Thomas-Fermi fission barriers of Myers and Swiatecki 53., using the topographic theorem of the same authors [18] to account for the contribution of the ground-state shell effect. Adjustments to measured barrier values [59] are applied. Details are described in Sec. III A

\section{Comparison with Experimental Data}

Figs. 30 to 35 show a survey on measured fission probabilities in comparison with the results of the GEF code. The data are taken from Refs. [158 163]. Some of the figures show the data from different reactions with different symbols. (See the original publications for details.)

\section{Discussion}

The absolute values and the energy dependence of the fission probabilities of most systems reaching from $\mathrm{Pa}$ to $\mathrm{Cm}$ are rather well reproduced by the GEF code at energies above the fission barrier. However, in many cases, fission sets in at too low energies in the calculation. In a few cases, the measured fission probabilities are considerably lower than the calculated ones, while the threshold and the energy dependence are rather similar. The most pronounced cases are ${ }^{229} \mathrm{Th},{ }^{230} \mathrm{Th},{ }^{231} \mathrm{Th},{ }^{233} \mathrm{Th}$, and ${ }^{234} \mathrm{U}$.

A possible key to the latter problem may be seen in the figures for ${ }^{231} \mathrm{~Pa},{ }^{235} \mathrm{~Np},{ }^{239} \mathrm{Pu},{ }^{240} \mathrm{Pu}$, and ${ }^{244} \mathrm{Cm}$, where different sets of measured data exist. In all these cases, one of the data sets gives appreciably higher values than the other one, and the higher values agree rather well with the model calculations. For fission probabilities obtained with transfer reactions, there may be a background originating from reactions on target contaminants (e.g. oxygen) or from other parasitic reactions like the breakup of the projectile (deuteron-breakup in particular). This may explain the differences encountered between the different groups of experimental data. Thus, this problem might have its origin in the experimental data at least in some of the cases. It may be noted that discrepancies between fission probabilities extracted by means of various entrance-channel reactions have recently been observed in Ref. 164.

The deviations near the threshold may be attributed to the shortcoming of the model due to its global description. As already mentioned in the introduction to this section, specific structural effects at low excitation energies, in particular structural information on the levels above the fission barrier are not properly considered. The observed deviations correspond to a shift of the effective threshold in the order of several $100 \mathrm{keV}$. Considering that the fission barriers extracted by different authors for the same nucleus often differ by $0.5 \mathrm{MeV}$ and more [59],
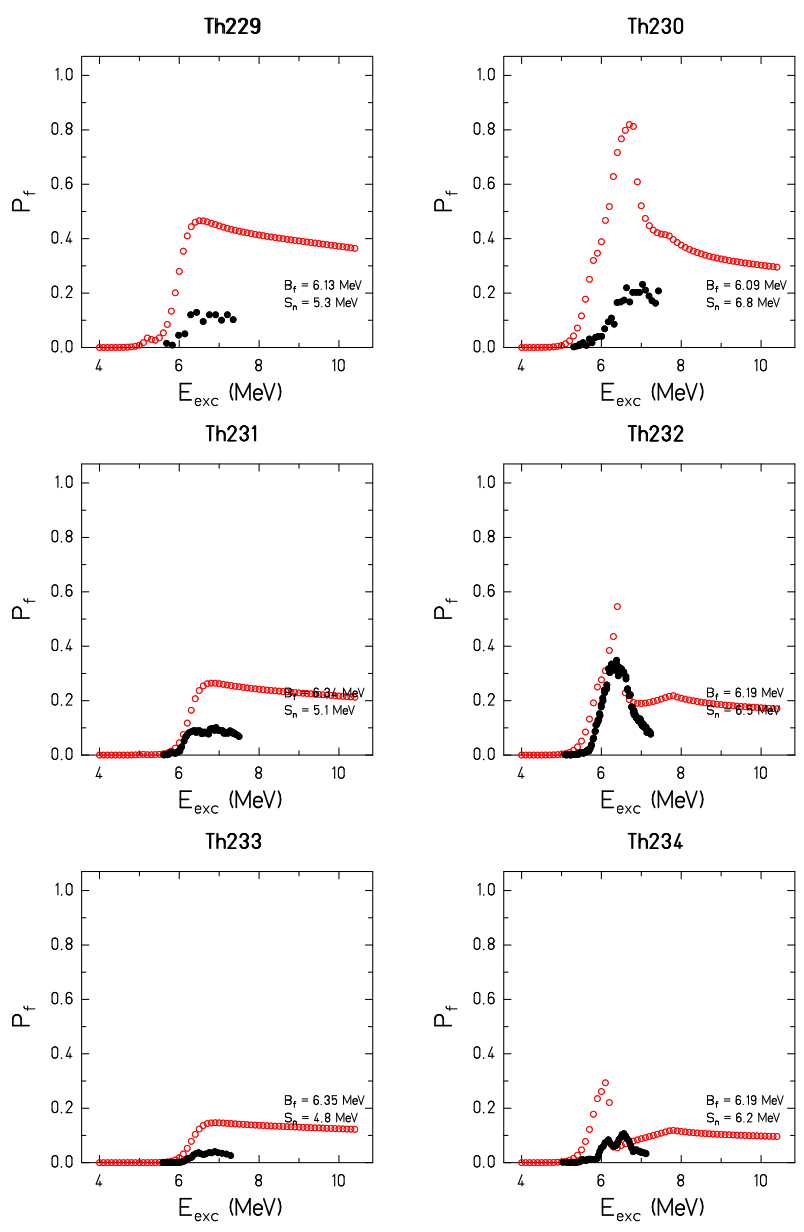

$\mathrm{Pa} 230$
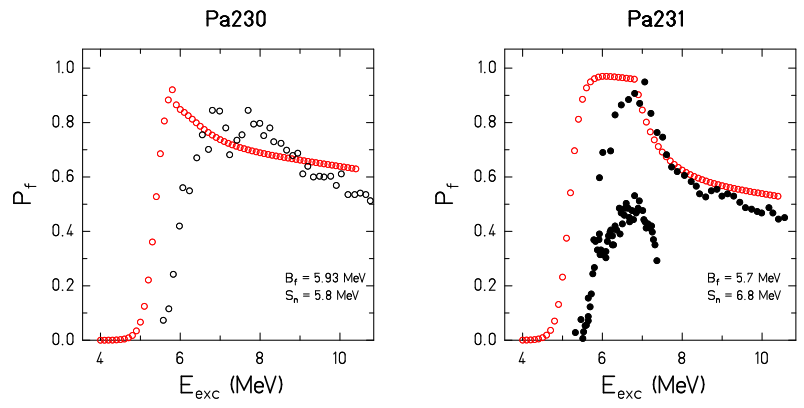

$\mathrm{Pa} 232$
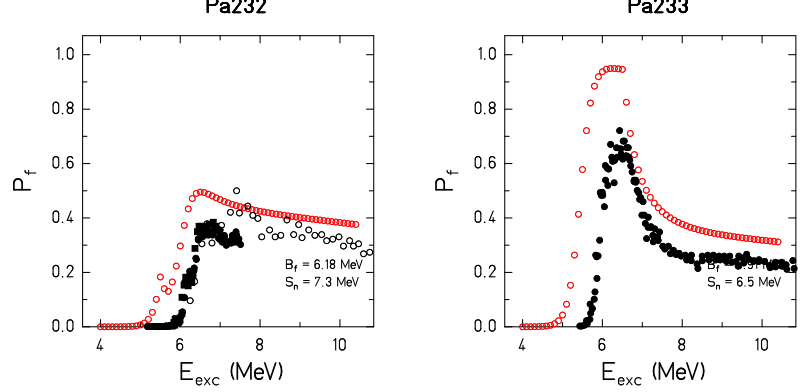

FIG. 30. (Color online) Benchmark of fission probabilities, part 1. Comparison of the measured fission probabilities (black symbols) with calculations with the GEF code (red symbols). The fission barrier and the neutron separation energy used in the calculations are listed. 

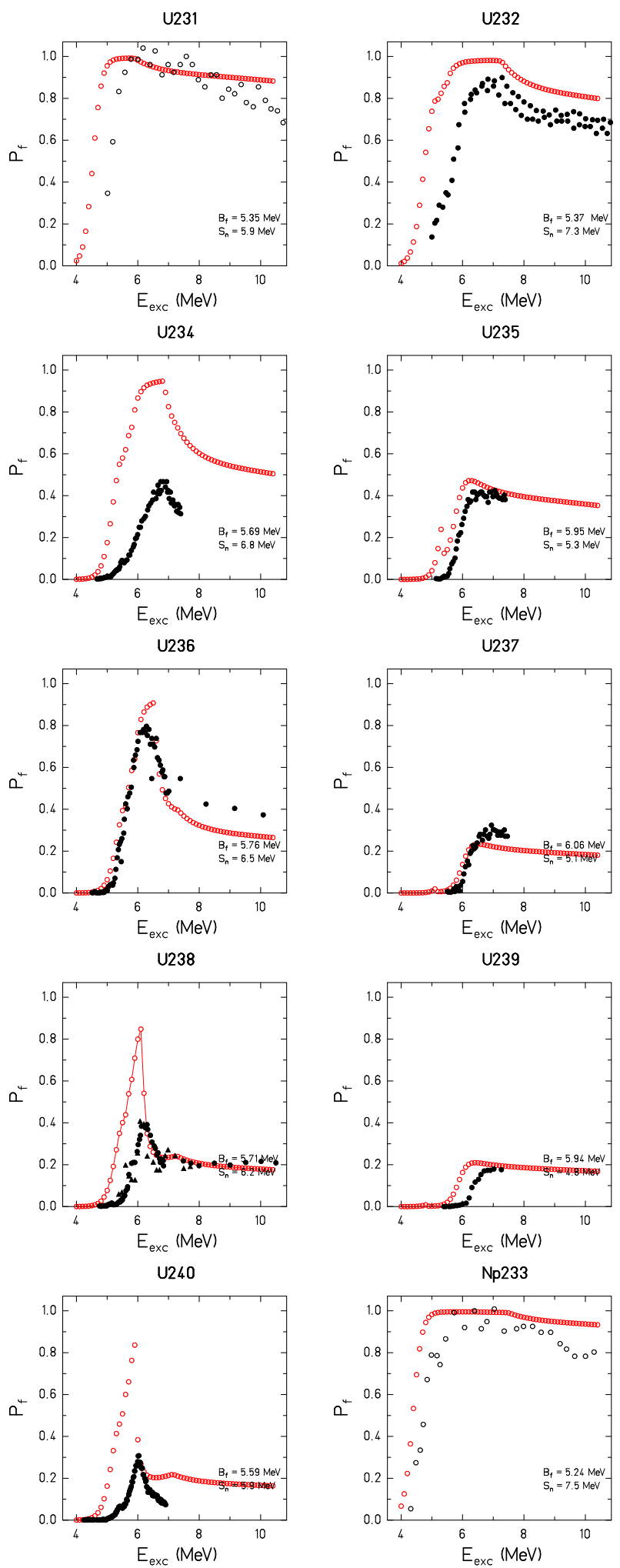

FIG. 31. (Color online) Benchmark of fission probabilities, part 2. Comparison of the measured fission probabilities (black symbols) with calculations with the GEF code (red symbols).
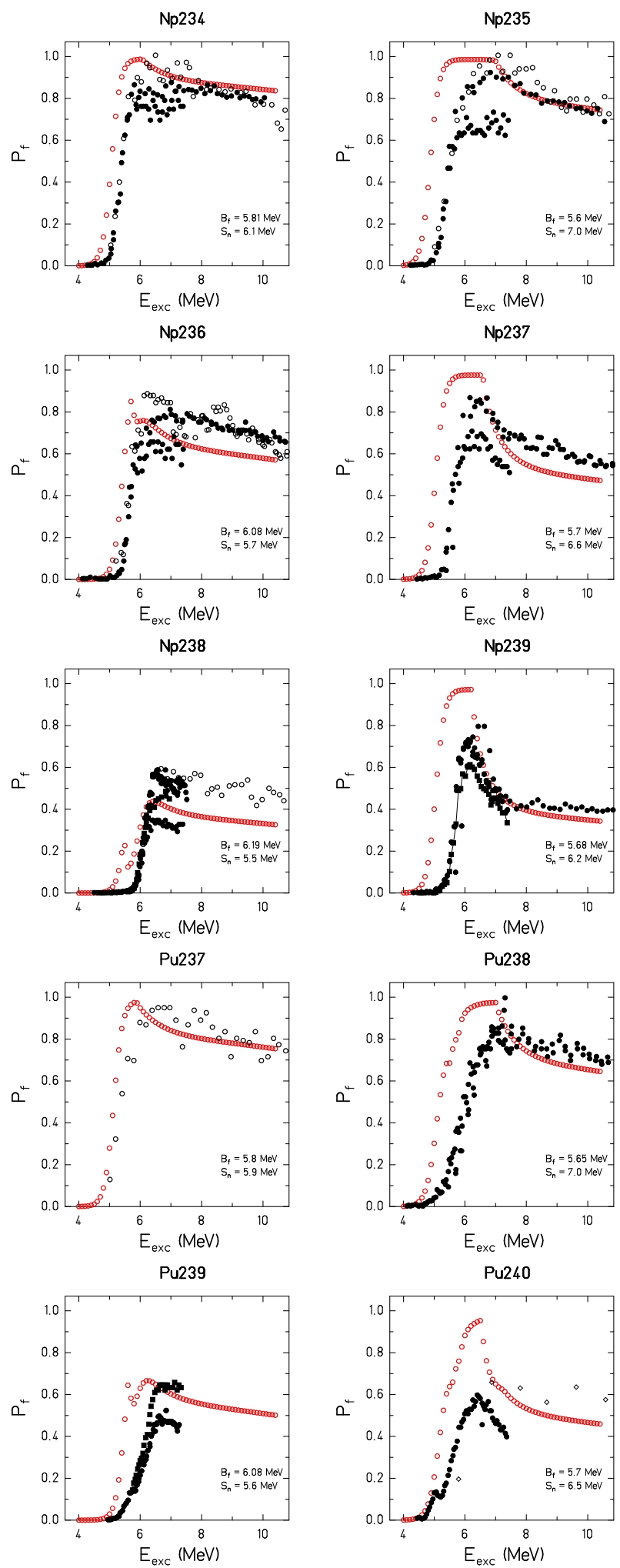

FIG. 32. (Color online) Benchmark of fission probabilities, part 3. Comparison of the measured fission probabilities (black symbols) with calculations with the GEF code (red symbols). 

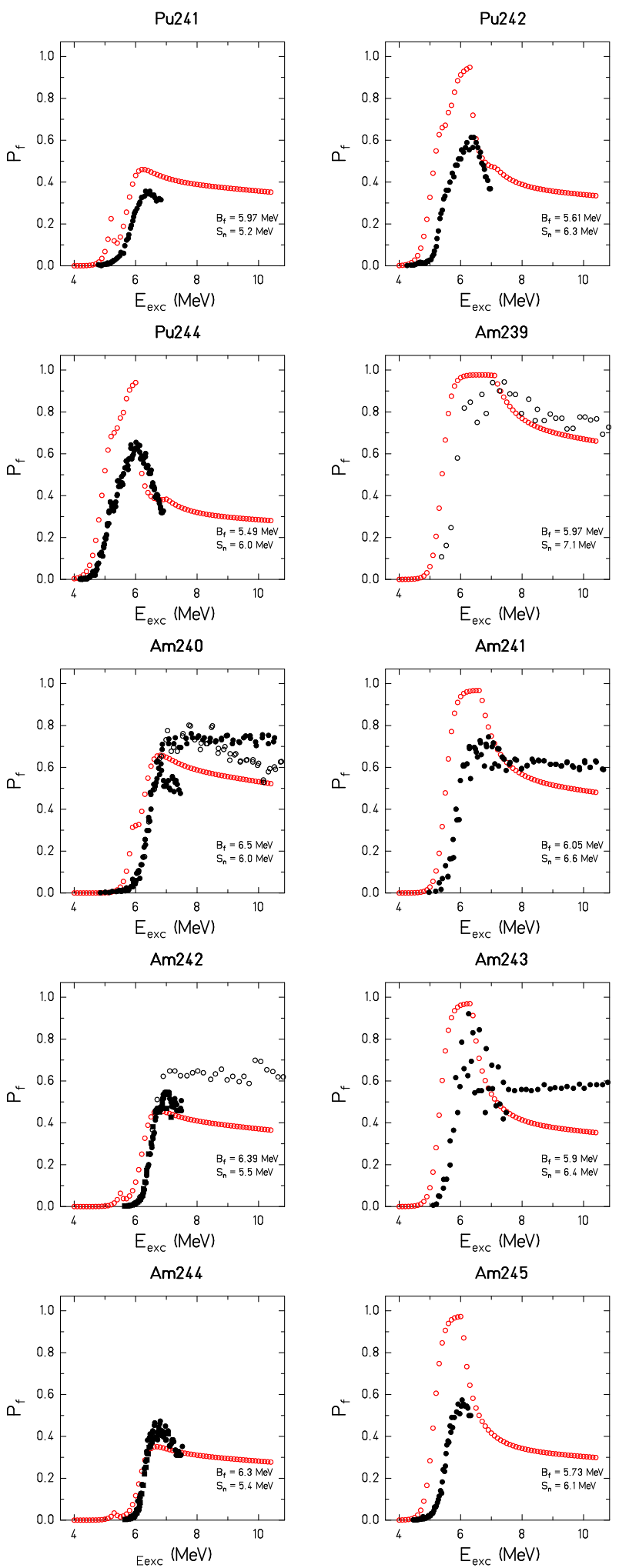

FIG. 33. (Color online) Benchmark of fission probabilities, part 4. Comparison of the measured fission probabilities (black symbols) with calculations with the GEF code (red symbols).
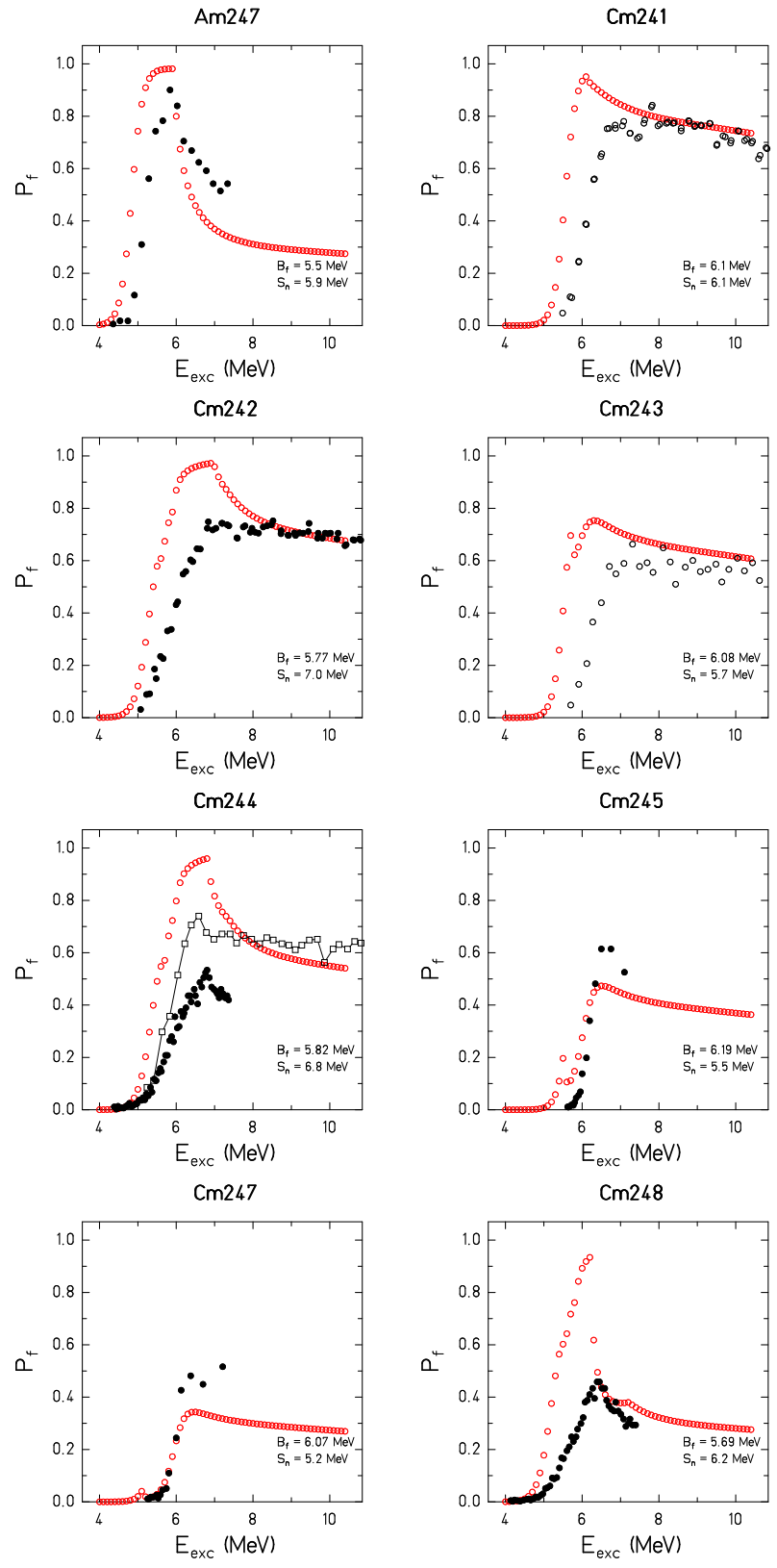

FIG. 34. (Color online) Benchmark of fission probabilities, part 5. Comparison of the measured fission probabilities (black symbols) with calculations with the GEF code (red symbols).

mostly due to differences in the assumed level densities especially at low excitation energies, the deviations may not be surprising. However, we are convinced that a shift of the fission barriers to reduce the deviations near the fission threshold is not the right way to solve this problem. The calculation of the fission probability in GEF is almost identical with the one used by Bjornholm and Lynn [57] with only one important difference: GEF does not take into account the detailed experimental informa- 

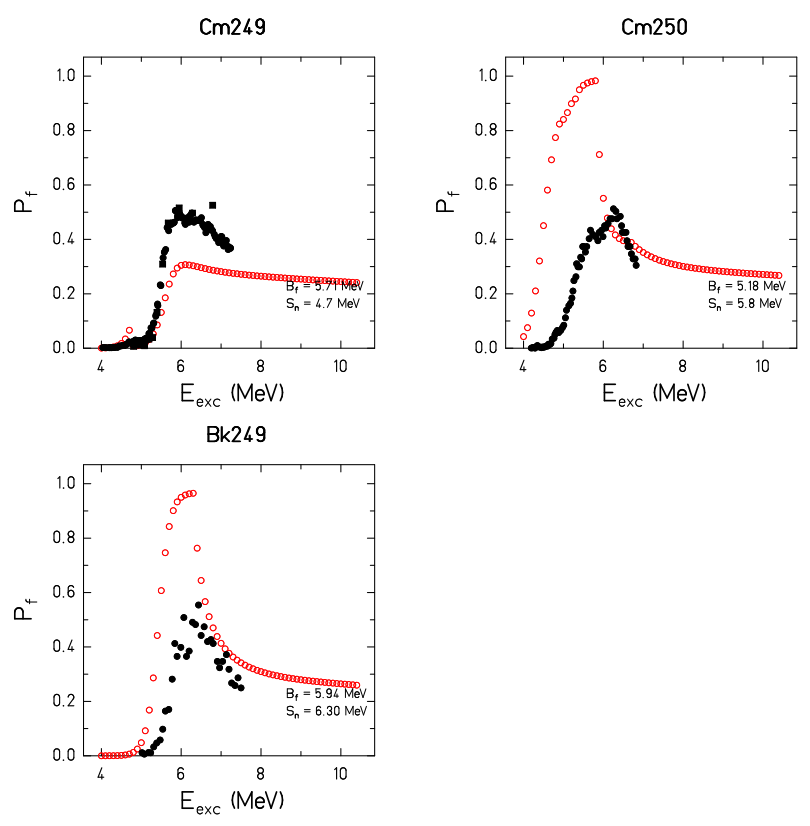

FIG. 35. (Color online) Benchmark of fission probabilities, part 6. Comparison of the measured fission probabilities (black symbols) with calculations with the GEF code (red symbols).

tion on excited states at low excitation energy (essentially those below the pairing gap) in the residue after neutron emission and at the barrier, because this information is not generally available in sufficient detail for the variety of nuclei covered by GEF. As already mentioned, the procedure used in Sec. III A for estimating the fission barriers by using the topographic theorem agrees perfectly with the results deduced by Bjornholm and Lynn from measured fission cross sections in a completely independent way. This good agreement for so many systems can hardly be an accident. It supports strongly the correctness of both approaches. Therefore, the fission barriers should not be modified, because this would distort the fission probabilities above the threshold region.

The kind of disagreement in the fission probabilities, in particular near the fission threshold, that is seen in the figures gives a realistic impression about the quality of the predictions of GEF that can be expected for cases, where no experimental data exist. The description of the fission probability near the threshold is certainly an aspect that deserves some improvement in the future development of the GEF model. Attempts to achieve this in a similar way as the global description of the energies of the yrast states presented in Sec. III J were not yet successful.

\section{Conclusion}

A global description of the fission probability of the actinides has been derived, which reproduces the experi- mental data above the threshold region rather well. Some discrepancies in the absolute values over the whole energy range might be caused by a background contribution due to the presence of light target contaminants in the experiment. The global description of the nuclear level densities near the ground state and near the fission threshold used in the code can only give a rather crude approximation of the behavior of the fission probability near the fission threshold. This explains the discrepancies in the fission probabilities near the fission threshold found for several systems. The energy-dependent fission probabilities are important to calculate the relative weights of the different fission chances at higher energies.

\section{B. Fission-fragment Yields}

\section{Introduction}

Several hundred different nuclides are produced in the fission of a heavy nucleus. They essentially contribute to the radioactive inventory of a fission reactor, and they are the source of most part of the decay heat that incurs in the fuel rods even long time after the shut-down of the reactor. The relative yields of the different nuclides depend on the fissioning nucleus and on the excitation energy at fission. Moreover, the radioactive-decay properties of the different fission products differ very much. Therefore, a very good knowledge on the yields of the different fission products is of paramount importance for the operation of a fission reactor and for the storage of used fuel rods.

New data are required when new generations of fission reactors are developed, e.g. when fission is induced by neutrons of higher energies, or when eventually other kind of fuel is used. Reliable model calculations of the fissionproduct yields are urgently required which can replace time-consuming and expensive experiments.

The GEF code [75, 165, 166] has been developed with the aim to provide this kind of information. In the following, the quality and the predictive power of the GEF code for calculating fission-fragment yields for different fissioning systems and a large range of energy is assessed.

\section{Experimental Techniques}

It is worthwhile to have a look at the different most often used experimental techniques applied to measure fission-product yields, because they determine the nature of the data.

The traditional approach is based on the identification of gamma rays that are characteristic for the radioactive decay of a specific fragment [167]. Fission-product masses after the emission of prompt neutrons are determined. This technique is able to identify the emitting nuclide unambiguously, but it requires additional knowledge on the decay properties, e.g. branching ratios, in order to deduce quantitative yields. Moreover, this tech- 



FIG. 36. (Color online) Evaluated and experimental mass distributions (black symbols) of fission fragments in comparison with the results of the GEF code (green and blue symbols) in a linear scale. Spontaneous fission, part 1. 

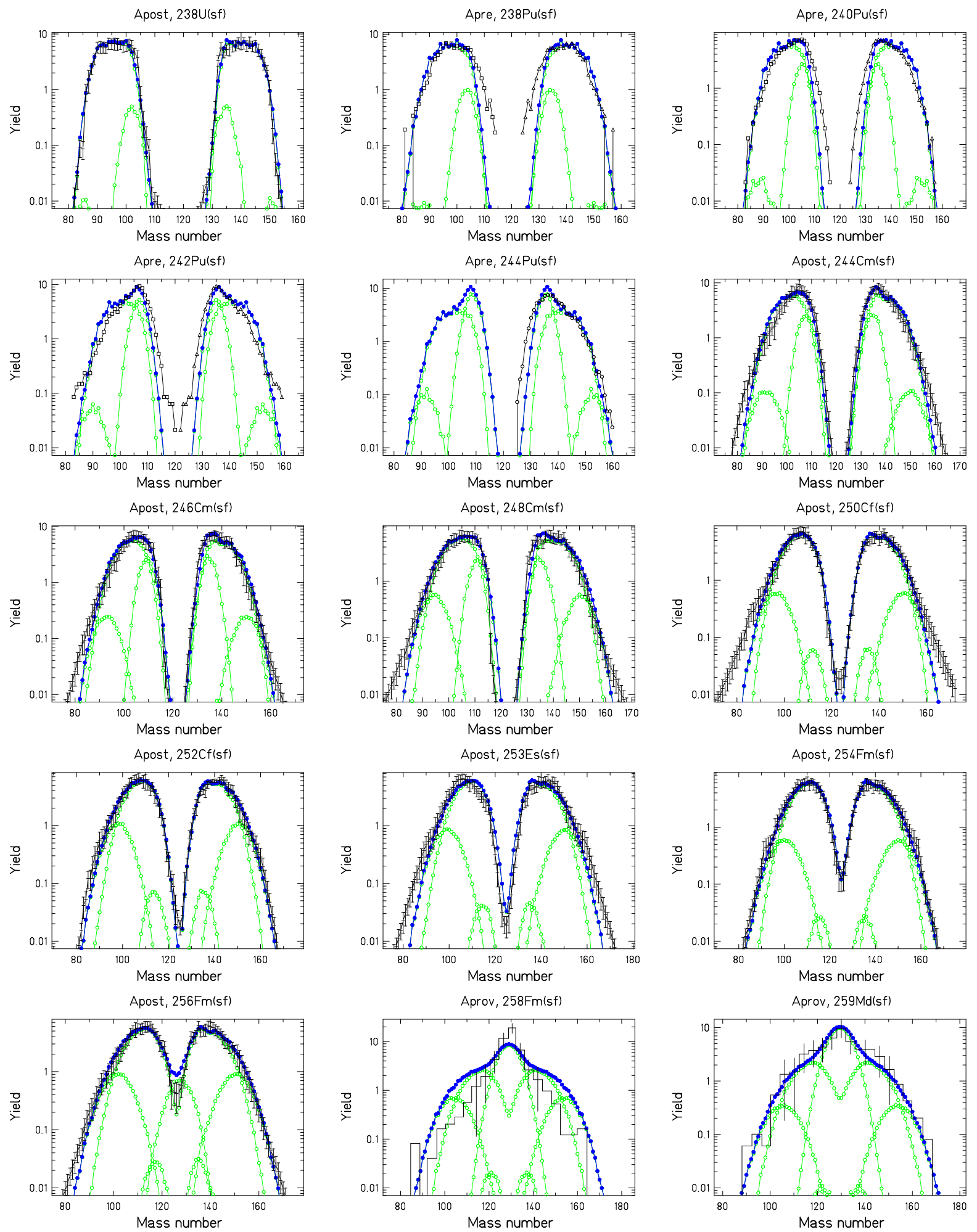

FIG. 37. (Color online) Evaluated and experimental mass distributions (black symbols) of fission fragments in comparison with the results of the GEF code (green and blue symbols) in a logarithmic scale. Spontaneous fission, part 1. 

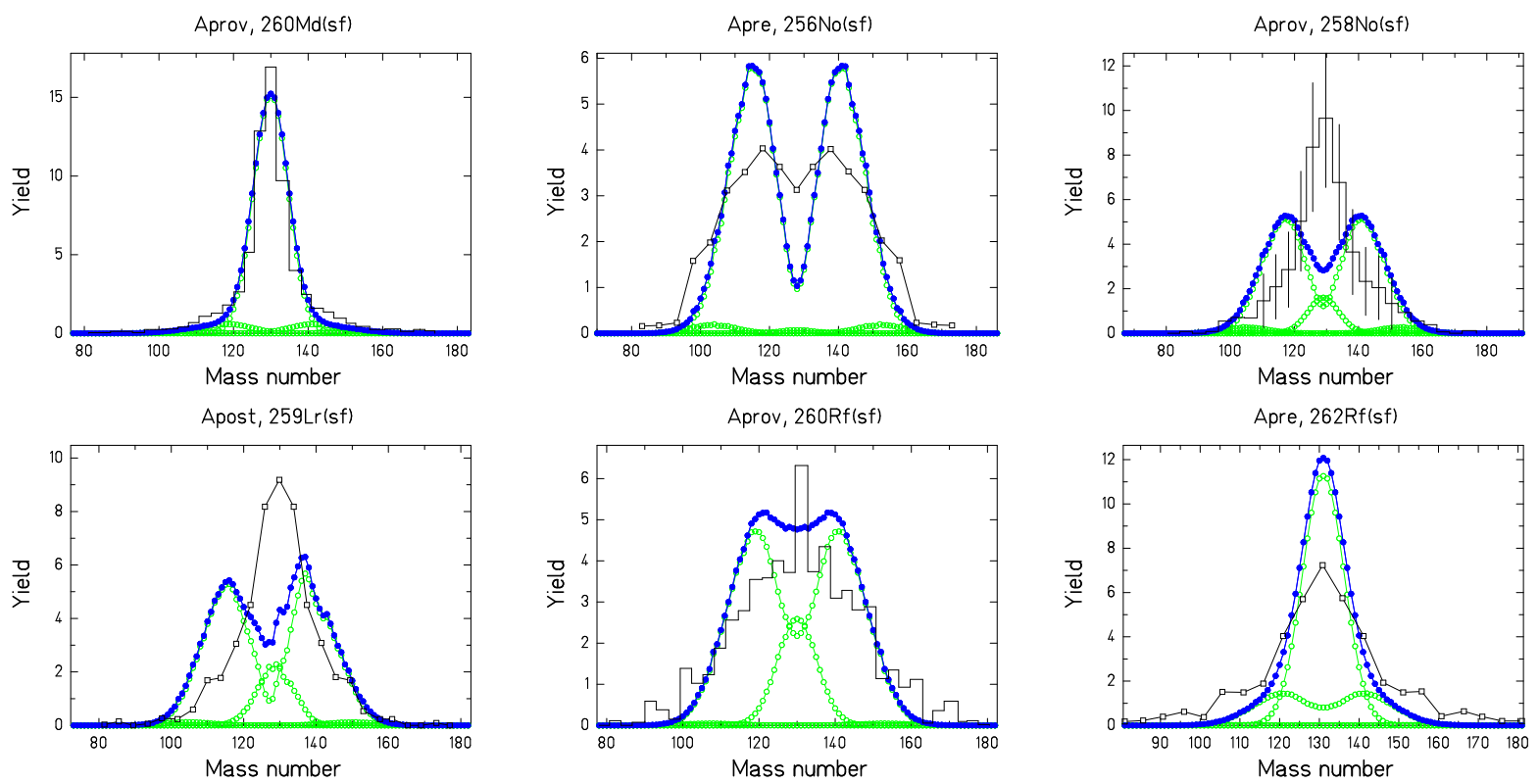

FIG. 38. (Color online) Evaluated and experimental mass distributions (black symbols) of fission fragments in comparison with the results of the GEF code (green and blue symbols) in a linear scale. Spontaneous fission, part 2.
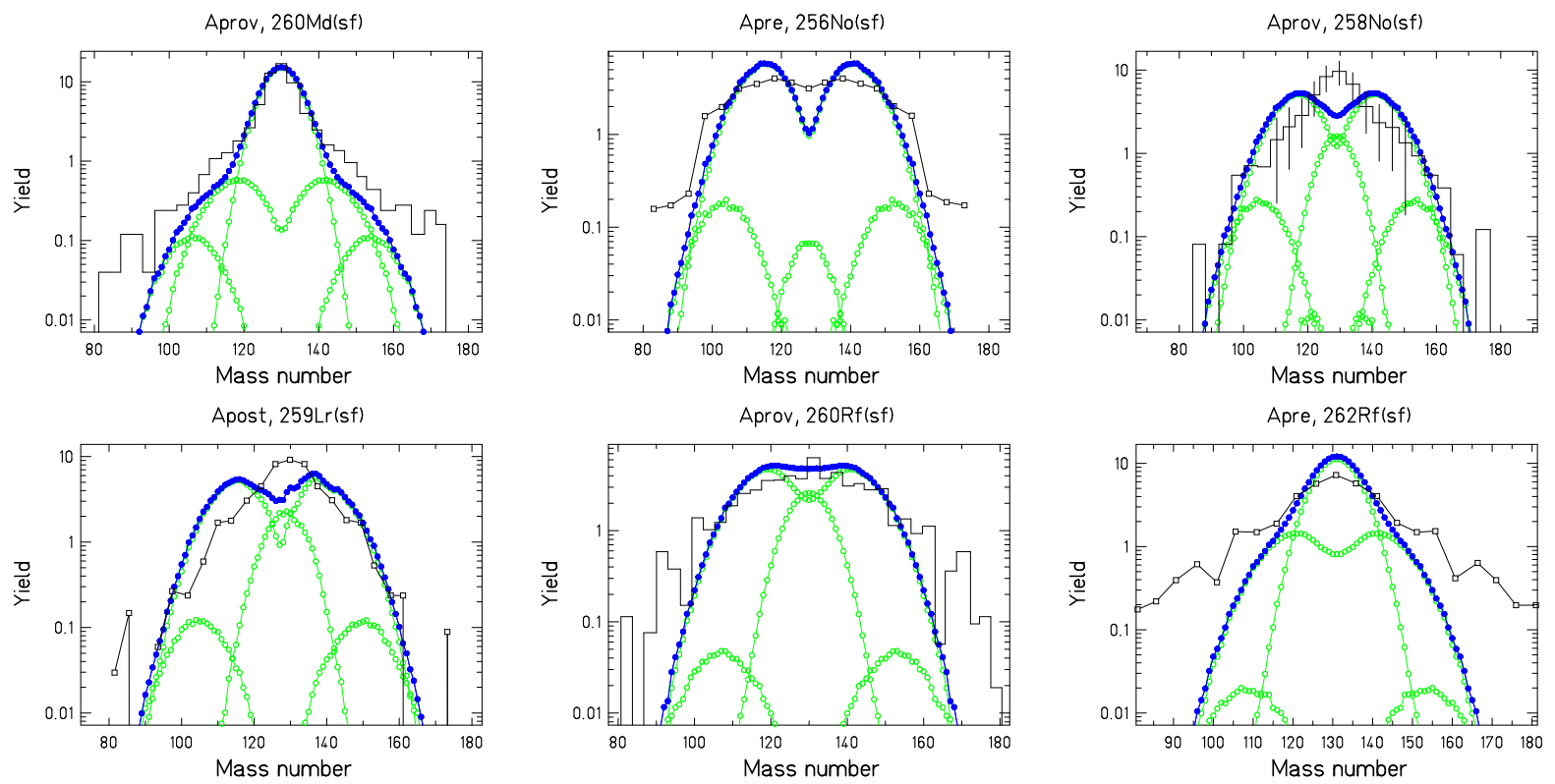

FIG. 39. (Color online) Evaluated and experimental mass distributions (black symbols) of fission fragments in comparison with the results of the GEF code (green and blue symbols) in a logarithmic scale. Spontaneous fission, part 2.

nique is not well suited for measuring the yields of shortlived fission products and unable to determine the yields of stable nuclides.

The masses of the fission products can also be determined by particle detectors that measure the energies and/or the velocities of the two fission products in coincidence [168 170]. These methods are suited for deducing the masses of the fission products before and after the emission of prompt neutrons. However, the resolution is not sufficient to determine the mass unambiguously in most cases. No information on the atomic number of the fission fragment can be obtained.

Unambiguous determination of fission-product masses has been achieved by use of the Lohengrin spectrograph 

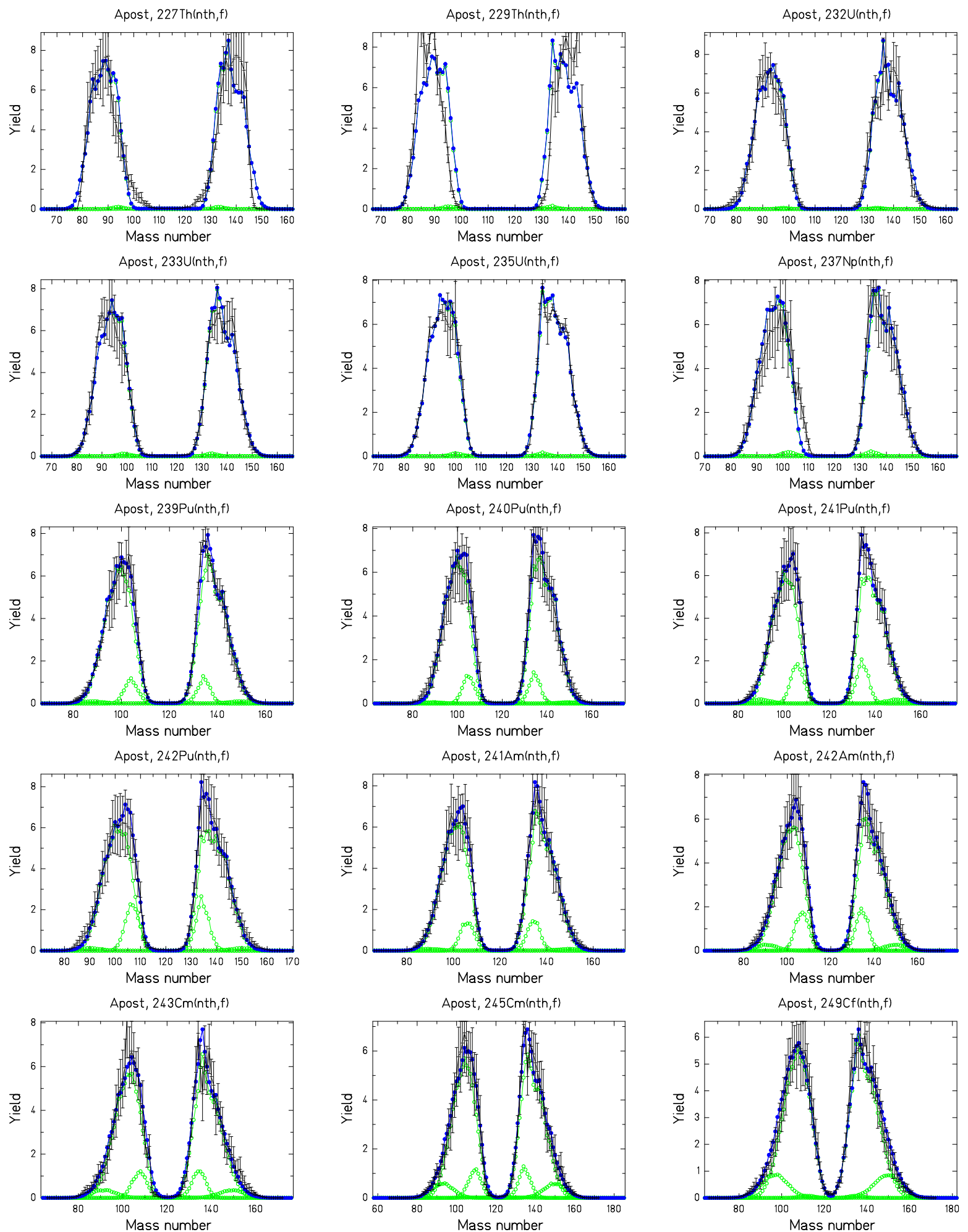

FIG. 40. (Color online) Evaluated and experimental mass distributions (black symbols) of fission fragments in comparison with the results of the GEF code (green and blue symbols) in a linear scale. Thermal-neutron-induced fission, part 1. 

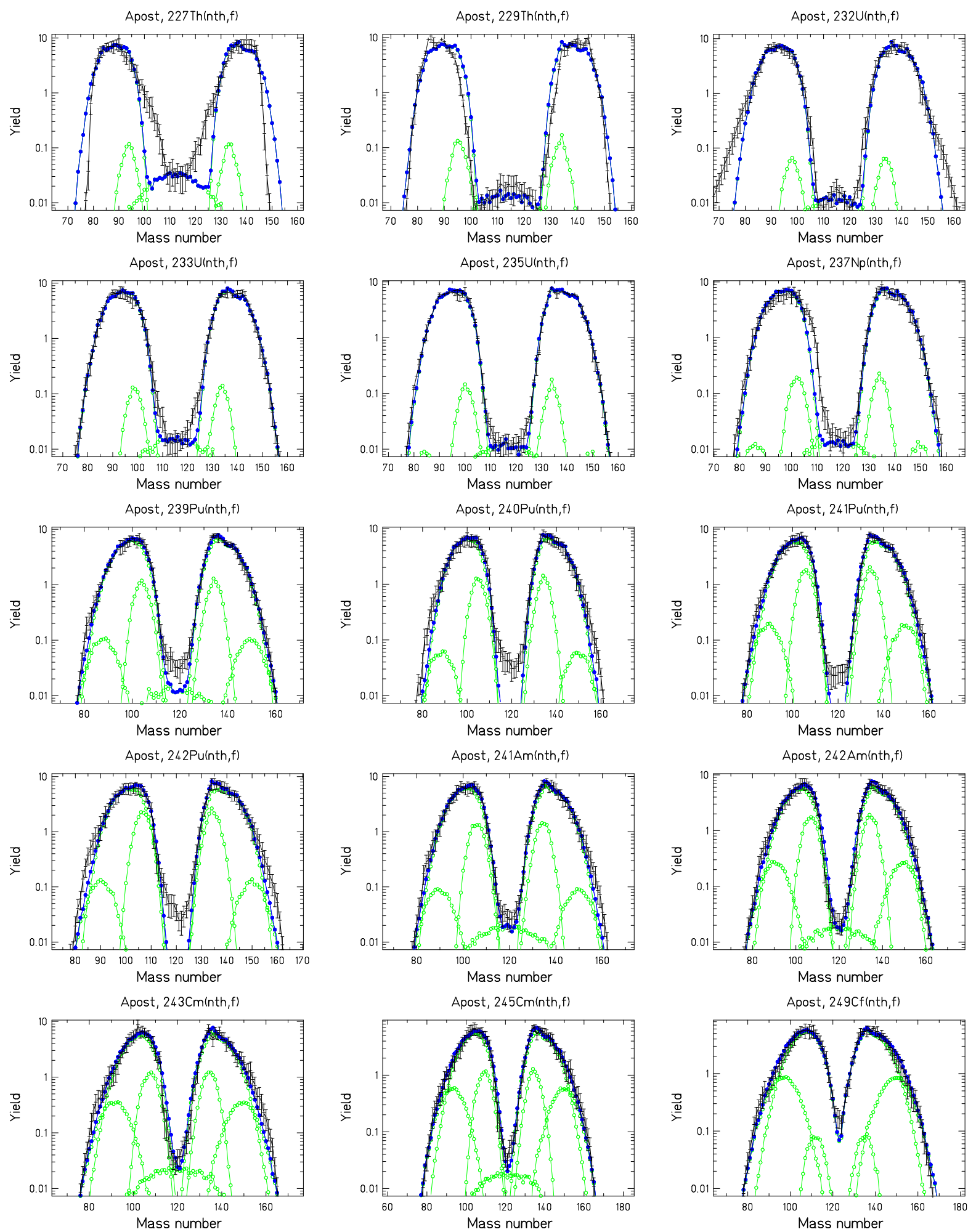

FIG. 41. (Color online) Evaluated and experimental mass distributions (black symbols) of fission fragments in comparison with the results of the GEF code (green and blue symbols) in a logarithmic scale. Thermal-neutron-induced fission, part 1. 

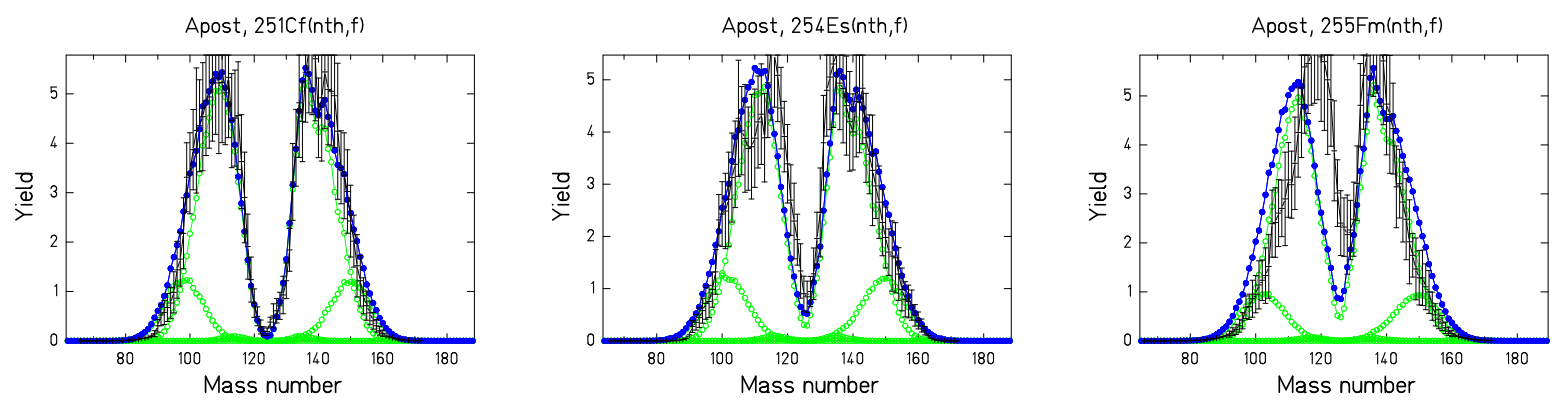

FIG. 42. (Color online) Evaluated and experimental mass distributions (black symbols) of fission fragments in comparison with the results of the GEF code (green and blue symbols) in a linear scale. Thermal-neutron-induced fission, part 2.
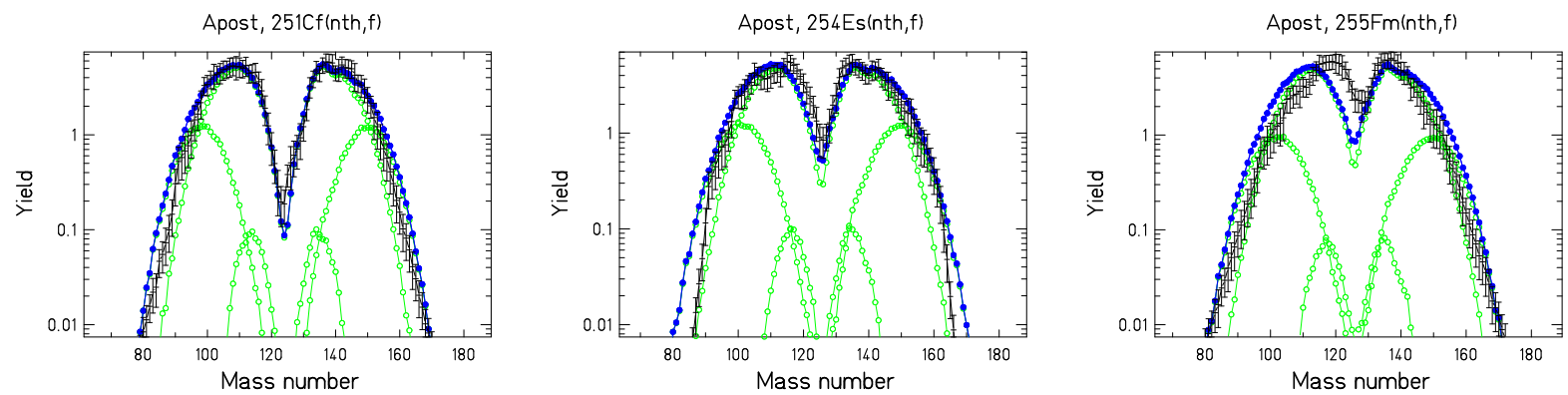

FIG. 43. (Color online) Evaluated and experimental mass distributions (black symbols) of fission fragments in comparison with the results of the GEF code (green and blue symbols) in a logarithmic scale. Thermal-neutron-induced fission, part 2.

[171] at the high-flux reactor of the ILL, Grenoble. Also, the nuclear charge in the light group of the fission products can be determined. However, this technique is restricted to thermal-neutron-induced fission and a limited choice of target material.

A novel kind of experiments in inverse kinematics [69, 172, 173] succeeded to determine the mass $A$ and the atomic number $Z$ of all fission products unambiguously in Coulomb fission of short-lived neutron-deficient projectile fragments at relativistic energies. The full identification of all fission products in $A$ and $Z$ was also achieved in the fission of transfer and fusion products from ${ }^{238} \mathrm{U}$ projectiles at energies above the Coulomb barrier [174]. Besides the unprecedented resolution in kinematical measurements, these experiments offer a wide choice of fissioning systems, not accessible before.

\section{Mass Distributions}

Fission-fragment mass distributions have a particular importance. Firstly, they are determined with full resolution for a large number of systems by gammaspectroscopic measurements. The data which are often incomplete are completed with the Wahl systematics [3] Moreover, the beta decay which is the predominant decay path follows the mass chain. Thus, the mass distribution can also be deduced from cumulative yields. Secondly, the mass distributions allow estimating the long-term radioactive decay characteristics rather well, because beta decay that connects nuclei with the same mass number is the predominant decay path in most cases.

In the following, experimental and evaluated mass distributions in four different energy classes and from different experimental sources are compared with the result of the GEF code. They are shown in logarithmic and in linear scale. The figures in logarithmic scale evidence the large variation of the yields over the whole mass range. For example, small peak-to-valley ratios and the contributions from weak fission channels can only be perceived in logarithmic scale, while deviations between empirical and calculated values in the dominant yields, where the relative empirical uncertainties are small, can be discerned much better in linear scale.

Depending on the experimental technique, mass distributions before emission of prompt neutrons $\left(A_{\text {pre }}\right)$ and after emission of prompt neutrons $\left(A_{\text {post }}\right)$ are given. In a few cases, $A_{\text {prov }}$, the provisional mass, is shown. It is directly deduced from the ratio of the kinetic energies $E_{1}$ and $E_{2}$ of the fragments, assuming that $A_{1} / A_{2}=E_{2} / E_{1}$, and, thus, it is not corrected for neutron emission.

The calculated individual contributions from the different fission modes are shown in addition as green symbols. The comparison is not exhaustive, but it gives a rather 

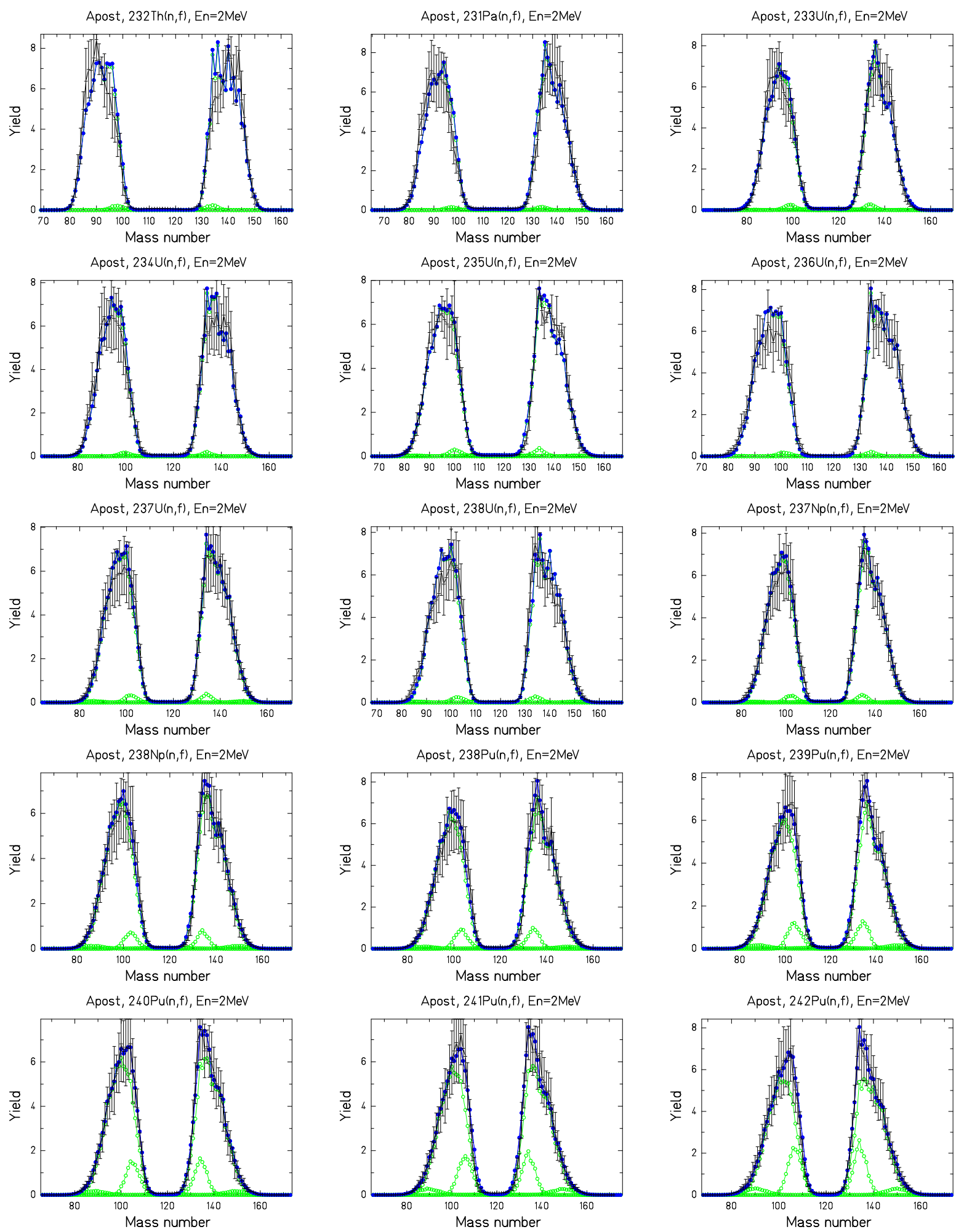

FIG. 44. (Color online) Evaluated and experimental mass distributions (black symbols) of fission fragments in comparison with the results of the GEF code (green and blue symbols) in a linear scale. Fast-neutron-induced fission, part 1. 

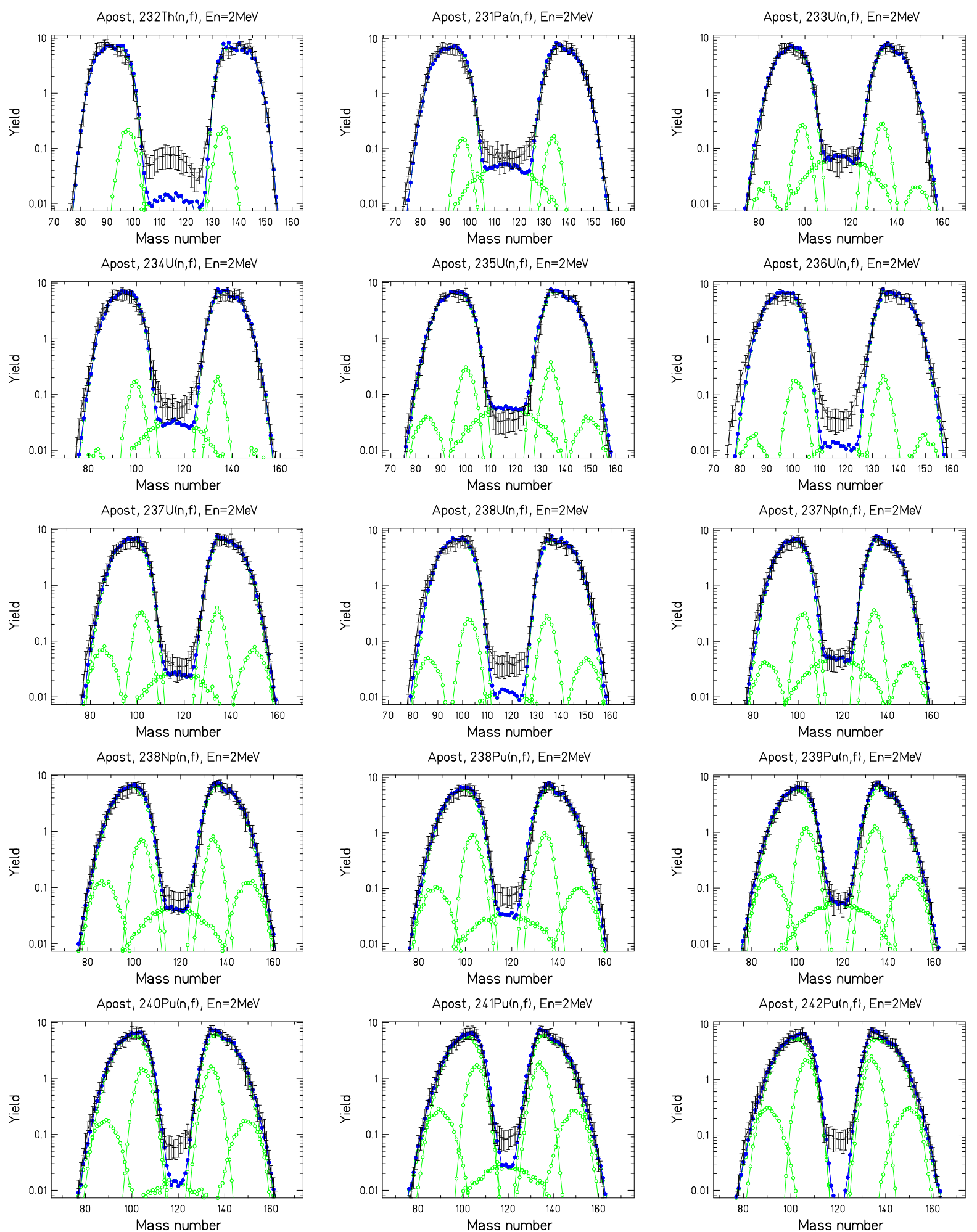

FIG. 45. (Color online) Evaluated and experimental mass distributions (black symbols) of fission fragments in comparison with the results of the GEF code (green and blue symbols) in a logarithmic scale. Fast-neutron-induced fission, part 1. 

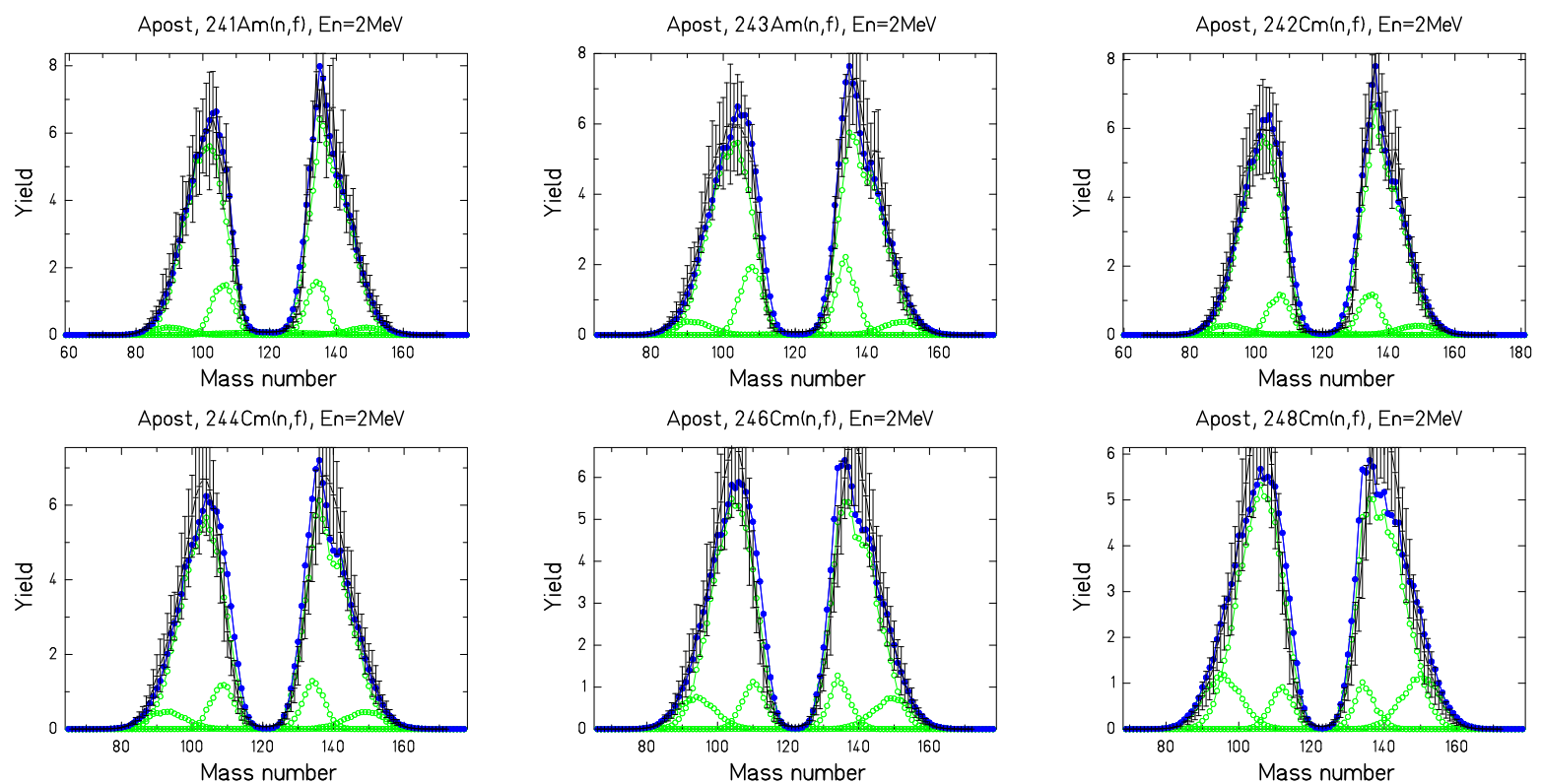

FIG. 46. (Color online) Evaluated and experimental mass distributions (black symbols) of fission fragments in comparison with the results of the GEF code (green and blue symbols) in a linear scale. Fast-neutron-induced fission, part 2.
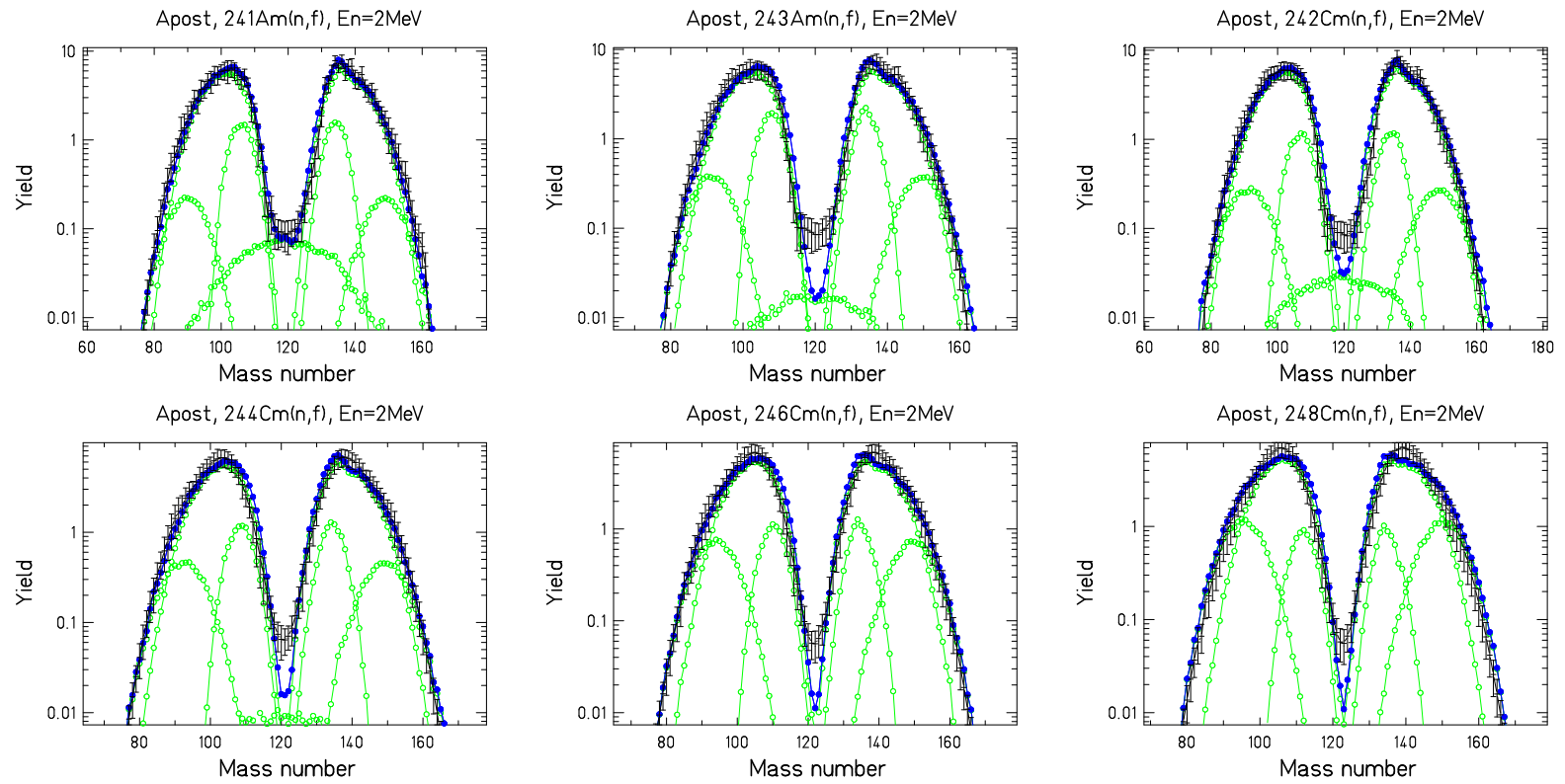

FIG. 47. (Color online) Evaluated and experimental mass distributions (black symbols) of fission fragments in comparison with the results of the GEF code (green and blue symbols) in a logarithmic scale. Fast-neutron-induced fission, part 2.

complete view on the variation of the mass distributions from protactinium to rutherfordium. The error bars represent the uncertainties given in the indicated references (see Tables VIII and IX).

Although the GEF code is able to produce uncertainties by calculations with perturbed parameters, they are not shown in order not to overload the figures. 

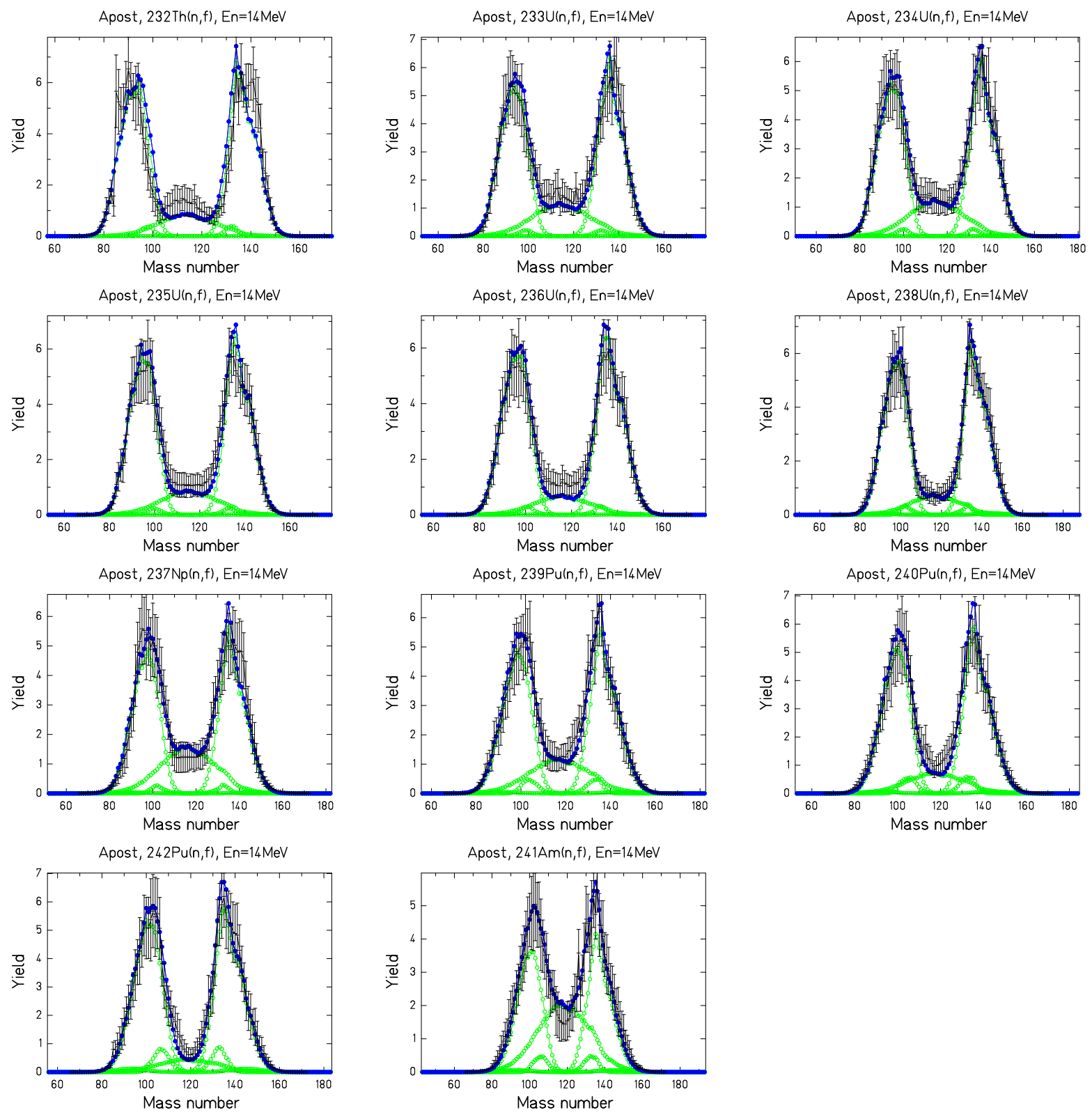

FIG. 48. (Color online) Evaluated and experimental mass distributions (black symbols) of fission fragments in comparison with the results of the GEF code (green and blue symbols) in a linear scale. 14-MeV-neutron-induced fission. 

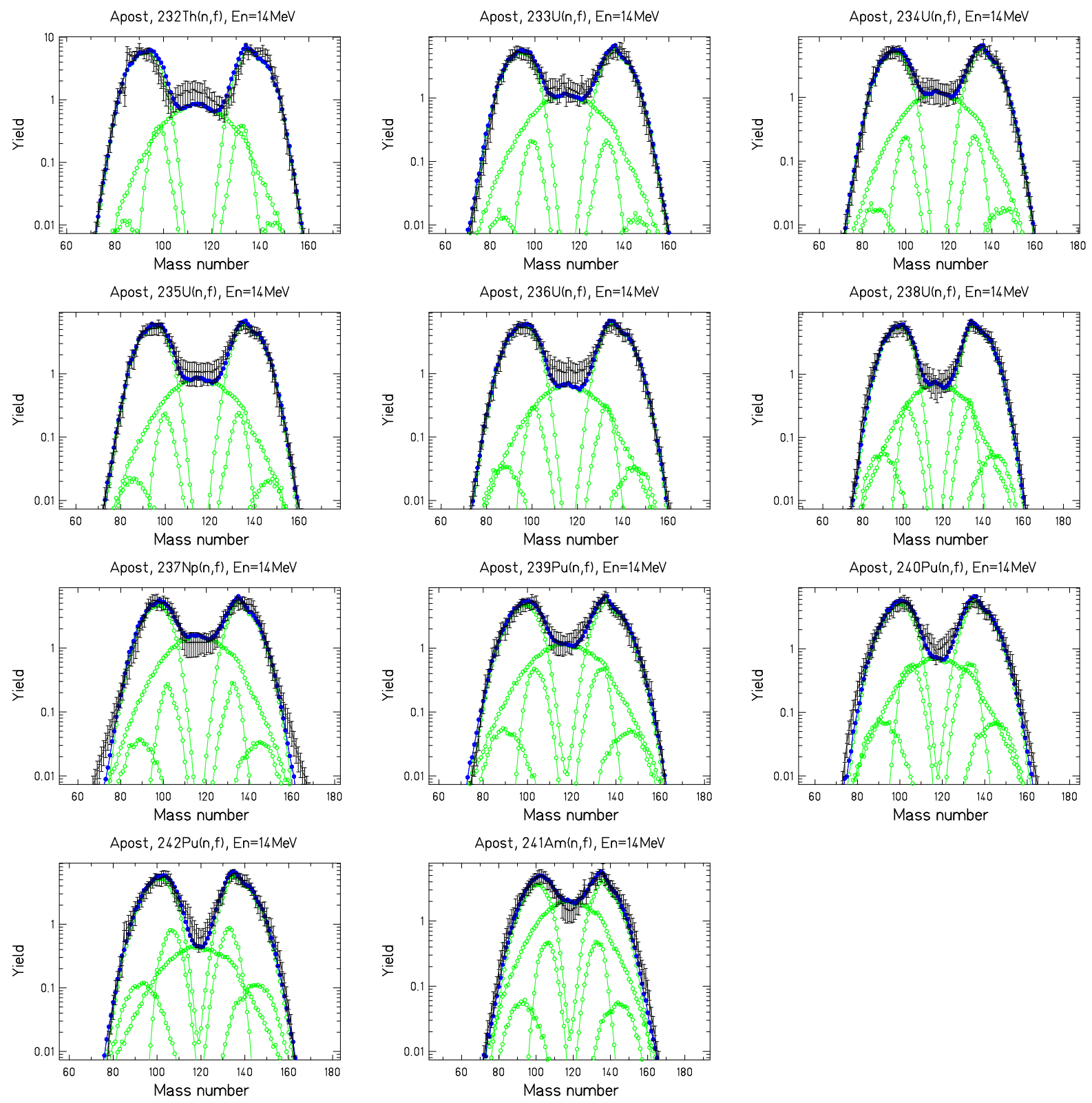

FIG. 49. (Color online) Evaluated and experimental mass distributions (black symbols) of fission fragments in comparison with the results of the GEF code (green and blue symbols) in a logarithmic scale. 14-MeV-neutron-induced fission. 
TABLE VIII. Measured and evaluated mass distributions, part 1 .

\begin{tabular}{|c|c|c|c|}
\hline System & $\begin{array}{c}\text { Measured } \\
\text { quantity }\end{array}$ & Reference & $\begin{array}{c}\text { reduced } \\
\text { Chi-squared }\end{array}$ \\
\hline${ }^{238} \mathrm{U}(\mathrm{sf})$ & $A_{\text {post }}$ & [179] & 3.2 \\
\hline${ }^{238} \mathrm{Pu}(\mathrm{sf})$ & $A_{\text {pre }}$ & [180] & - \\
\hline${ }^{240} \mathrm{Pu}(\mathrm{sf})$ & $A_{\text {pre }}$ & [180] & - \\
\hline${ }^{242} \mathrm{Pu}(\mathrm{sf})$ & $A_{\text {pre }}$ & [180] & - \\
\hline${ }^{244} \mathrm{Pu}(\mathrm{sf})$ & $A_{\text {pre }}$ & 181 & - \\
\hline${ }^{244} \mathrm{Cm}(\mathrm{sf})$ & $A_{\text {post }}$ & [179] & 1.4 \\
\hline${ }^{246} \mathrm{Cm}(\mathrm{sf})$ & $A_{\text {post }}$ & [179] & 1.1 \\
\hline${ }^{248} \mathrm{Cm}(\mathrm{sf})$ & $A_{\text {post }}$ & [179] & 7.8 \\
\hline${ }^{250} \mathrm{Cf}(\mathrm{sf})$ & $A_{\text {post }}$ & [179] & 0.9 \\
\hline${ }^{252} \mathrm{Cf}(\mathrm{sf})$ & $A_{\text {post }}$ & [179] & 0.6 \\
\hline${ }^{253} \operatorname{Es}(\mathrm{sf})$ & $A_{\text {post }}$ & [179] & 3.7 \\
\hline${ }^{254} \mathrm{Fm}(\mathrm{sf})$ & $A_{\text {post }}$ & [179] & 0.4 \\
\hline${ }^{256} \mathrm{Fm}(\mathrm{sf})$ & $A_{\text {post }}$ & [179] & 0.8 \\
\hline${ }^{258} \mathrm{Fm}(\mathrm{sf})$ & $A_{\text {prov }}$ & 182 & - \\
\hline${ }^{259} \mathrm{Md}(\mathrm{sf})$ & $A_{\text {prov }}$ & 182 & 一 \\
\hline${ }^{260} \mathrm{Md}(\mathrm{sf})$ & $A_{\text {prov }}$ & [182] & - \\
\hline${ }^{256} \mathrm{No}(\mathrm{sf})$ & $A_{\text {pre }}$ & [183] & - \\
\hline${ }^{258} \mathrm{No}(\mathrm{sf})$ & $A_{\text {prov }}$ & [182] & - \\
\hline${ }^{259} \operatorname{Lr}(\mathrm{sf})$ & $A_{\text {post }}$ & 184 & 一 \\
\hline${ }^{260} \mathrm{Rf}(\mathrm{sf})$ & $A_{\text {prov }}$ & {$[182]$} & - \\
\hline${ }^{262} \mathrm{Rf}(\mathrm{sf})$ & $A_{\text {pre }}$ & [185] & - \\
\hline${ }^{227} \operatorname{Th}\left(\mathrm{n}_{\mathrm{th}}, \mathrm{f}\right)$ & $A_{\text {post }}$ & [179] & 420 \\
\hline${ }^{229} \operatorname{Th}\left(\mathrm{n}_{\mathrm{th}}, \mathrm{f}\right)$ & $A_{\text {post }}$ & [179] & 26 \\
\hline${ }^{232} \mathrm{U}\left(\mathrm{n}_{\mathrm{th}}, \mathrm{f}\right)$ & $A_{\text {post }}$ & [179] & 1.6 \\
\hline${ }^{233} \mathrm{U}\left(\mathrm{n}_{\mathrm{th}}, \mathrm{f}\right)$ & $A_{\text {post }}$ & [179] & 1.2 \\
\hline${ }^{235} \mathrm{U}\left(\mathrm{n}_{\mathrm{th}}, \mathrm{f}\right)$ & $A_{\text {post }}$ & [179] & 7.4 \\
\hline${ }^{237} \mathrm{~Np}\left(\mathrm{n}_{\mathrm{th}}, \mathrm{f}\right)$ & $A_{\text {post }}$ & [179] & 1.1 \\
\hline${ }^{239} \mathrm{Pu}\left(\mathrm{n}_{\mathrm{th}}, \mathrm{f}\right)$ & $A_{\text {post }}$ & [179] & 1.2 \\
\hline${ }^{240} \mathrm{Pu}\left(\mathrm{n}_{\mathrm{th}}, \mathrm{f}\right)$ & $A_{\text {post }}$ & [179] & 0.9 \\
\hline${ }^{241} \mathrm{Pu}\left(\mathrm{n}_{\mathrm{th}}, \mathrm{f}\right)$ & $A_{\text {post }}$ & [179] & 0.7 \\
\hline${ }^{242} \mathrm{Pu}\left(\mathrm{n}_{\mathrm{th}}, \mathrm{f}\right)$ & $A_{\text {post }}$ & [179] & 0.8 \\
\hline${ }^{241} \mathrm{Am}\left(\mathrm{n}_{\mathrm{th}}, \mathrm{f}\right)$ & $A_{\text {post }}$ & [179] & 0.6 \\
\hline${ }^{242} \mathrm{Am}\left(\mathrm{n}_{\mathrm{th}}, \mathrm{f}\right)$ & $A_{\text {post }}$ & [179] & 0.9 \\
\hline${ }^{243} \mathrm{Cm}\left(\mathrm{n}_{\mathrm{th}}, \mathrm{f}\right)$ & $A_{\text {post }}$ & [179] & 1.7 \\
\hline${ }^{245} \mathrm{Cm}\left(\mathrm{n}_{\mathrm{th}}, \mathrm{f}\right)$ & $A_{\text {post }}$ & [179] & 0.5 \\
\hline${ }^{249} \mathrm{Cf}\left(\mathrm{n}_{\mathrm{th}}, \mathrm{f}\right)$ & $A_{\text {post }}$ & [179] & 0.7 \\
\hline${ }^{251} \mathrm{Cf}\left(\mathrm{n}_{\mathrm{th}}, \mathrm{f}\right)$ & $A_{\text {post }}$ & [179] & 4.9 \\
\hline${ }^{254} \mathrm{Es}\left(\mathrm{n}_{\mathrm{th}}, \mathrm{f}\right)$ & $A_{\text {post }}$ & [179] & 9.5 \\
\hline${ }^{255} \mathrm{Fm}\left(\mathrm{n}_{\mathrm{th}}, \mathrm{f}\right)$ & $A_{\text {post }}$ & [179] & 5.6 \\
\hline
\end{tabular}

Note: Measured and evaluated mass distributions used for the comparison in Figs. 36 to 49, their nature and their references. The last column gives the sum of the squared deviations of the GEF results from the evaluated yields divided by the uncertainties of the empirical data per degrees of freedom (reduced Chi-squared). This value should be around 1 for a good description. Only yields larger than $0.01 \%$ have been considered.

a. Spontaneous Fission: Figs. 36 to 39 show the calculated mass distributions for spontaneous fission in comparison with measured or evaluated data of a number of systems in linear and in logarithmic scale. Although spontaneous fission is less important for technical applications, these figures are essential for revealing the de-
TABLE IX. Measured and evaluated mass distributions, part 2 .

\begin{tabular}{|c|c|c|c|}
\hline System & $\begin{array}{c}\text { Measured } \\
\text { quantity }\end{array}$ & Reference & $\begin{array}{c}\text { reduced } \\
\text { Chi-squared }\end{array}$ \\
\hline${ }^{232} \operatorname{Th}(\mathrm{n}, \mathrm{f}), \mathrm{E}_{n}=$ fast & $A_{\text {post }}$ & [179] & 1.7 \\
\hline${ }^{231} \mathrm{~Pa}(\mathrm{n}, \mathrm{f}), \mathrm{E}_{n}=$ fast & $A_{\text {post }}$ & [179] & 1.0 \\
\hline${ }^{233} \mathrm{U}(\mathrm{n}, \mathrm{f}), \mathrm{E}_{n}=$ fast & $A_{\text {post }}$ & [179] & 1.0 \\
\hline${ }^{234} \mathrm{U}(\mathrm{n}, \mathrm{f}), \mathrm{E}_{n}=$ fast & $A_{\text {post }}$ & [179] & 1.0 \\
\hline${ }^{235} \mathrm{U}(\mathrm{n}, \mathrm{f}), \mathrm{E}_{n}=$ fast & $A_{\text {post }}$ & [179] & 1.5 \\
\hline${ }^{236} \mathrm{U}(\mathrm{n}, \mathrm{f}), \mathrm{E}_{n}=$ fast & $A_{\text {post }}$ & [179] & 1.2 \\
\hline${ }^{237} \mathrm{U}(\mathrm{n}, \mathrm{f}), \mathrm{E}_{n}=$ fast & $A_{\text {post }}$ & [179] & 0.4 \\
\hline${ }^{238} \mathrm{U}(\mathrm{n}, \mathrm{f}), \mathrm{E}_{n}=$ fast & $A_{\text {post }}$ & [179] & 1.1 \\
\hline${ }^{237} \mathrm{~Np}(\mathrm{n}, \mathrm{f}), \mathrm{E}_{n}=$ fast & $A_{\text {post }}$ & [179] & 0.5 \\
\hline${ }^{238} \mathrm{~Np}(\mathrm{n}, \mathrm{f}), \mathrm{E}_{n}=$ fast & $A_{\text {post }}$ & [179] & 0.5 \\
\hline${ }^{238} \mathrm{Pu}(\mathrm{n}, \mathrm{f}), \mathrm{E}_{n}=$ fast & $A_{\text {post }}$ & [179] & 0.7 \\
\hline${ }^{239} \mathrm{Pu}(\mathrm{n}, \mathrm{f}), \mathrm{E}_{n}=$ fast & $A_{\text {post }}$ & [179] & 0.6 \\
\hline${ }^{240} \mathrm{Pu}(\mathrm{n}, \mathrm{f}), \mathrm{E}_{n}=$ fast & $A_{\text {post }}$ & [179] & 0.6 \\
\hline${ }^{241} \mathrm{Pu}(\mathrm{n}, \mathrm{f}), \mathrm{E}_{n}=$ fast & $A_{\text {post }}$ & [179] & 0.7 \\
\hline${ }^{242} \mathrm{Pu}(\mathrm{n}, \mathrm{f}), \mathrm{E}_{n}=$ fast & $A_{\text {post }}$ & [179] & 0.6 \\
\hline${ }^{241} \operatorname{Am}(\mathrm{n}, \mathrm{f}), \mathrm{E}_{n}=$ fast & $A_{\text {post }}$ & [179] & 0.7 \\
\hline${ }^{243} \operatorname{Am}(\mathrm{n}, \mathrm{f}), \mathrm{E}_{n}=$ fast & $A_{\text {post }}$ & [179] & 1.4 \\
\hline${ }^{242} \mathrm{Cm}(\mathrm{n}, \mathrm{f}), \mathrm{E}_{n}=$ fast & $A_{\text {post }}$ & [179] & 1.4 \\
\hline${ }^{244} \mathrm{Cm}(\mathrm{n}, \mathrm{f}), \mathrm{E}_{n}=$ fast & $A_{\text {post }}$ & [179] & 1.0 \\
\hline${ }^{246} \mathrm{Cm}(\mathrm{n}, \mathrm{f}), \mathrm{E}_{n}=$ fast & $A_{\text {post }}$ & [179] & 1.0 \\
\hline${ }^{248} \mathrm{Cm}(\mathrm{n}, \mathrm{f}), \mathrm{E}_{n}=$ fast & $A_{\text {post }}$ & [179] & 1.5 \\
\hline${ }^{232} \operatorname{Th}(\mathrm{n}, \mathrm{f}), \mathrm{E}_{n}=14 \mathrm{MeV}$ & $A_{\text {post }}$ & [179] & 1.7 \\
\hline${ }^{233} \mathrm{U}(\mathrm{n}, \mathrm{f}), \mathrm{E}_{n}=14 \mathrm{MeV}$ & $A_{\text {post }}$ & [179] & 0.7 \\
\hline${ }^{234} \mathrm{U}(\mathrm{n}, \mathrm{f}), \mathrm{E}_{n}=14 \mathrm{MeV}$ & $A_{\text {post }}$ & [179] & 0.3 \\
\hline${ }^{235} \mathrm{U}(\mathrm{n}, \mathrm{f}), \mathrm{E}_{n}=14 \mathrm{MeV}$ & $A_{\text {post }}$ & [179] & 0.5 \\
\hline${ }^{236} \mathrm{U}(\mathrm{n}, \mathrm{f}), \mathrm{E}_{n}=14 \mathrm{MeV}$ & $A_{\text {post }}$ & [179] & 0.4 \\
\hline${ }^{238} \mathrm{U}(\mathrm{n}, \mathrm{f}), \mathrm{E}_{n}=14 \mathrm{MeV}$ & $A_{\text {post }}$ & [179] & 0.3 \\
\hline${ }^{237} \mathrm{~Np}(\mathrm{n}, \mathrm{f}), \mathrm{E}_{n}=14 \mathrm{MeV}$ & $A_{\text {post }}$ & [179] & 0.6 \\
\hline${ }^{239} \mathrm{Pu}(\mathrm{n}, \mathrm{f}), \mathrm{E}_{n}=14 \mathrm{MeV}$ & $A_{\text {post }}$ & [179] & 0.5 \\
\hline${ }^{240} \mathrm{Pu}(\mathrm{n}, \mathrm{f}), \mathrm{E}_{n}=14 \mathrm{MeV}$ & $A_{\text {post }}$ & [179] & 0.7 \\
\hline${ }^{242} \mathrm{Pu}(\mathrm{n}, \mathrm{f}), \mathrm{E}_{n}=14 \mathrm{MeV}$ & $A_{\text {post }}$ & [179] & 0.5 \\
\hline${ }^{241} \mathrm{Am}(\mathrm{n}, \mathrm{f}), \mathrm{E}_{n}=14 \mathrm{MeV}$ & $A_{\text {post }}$ & [179] & 0.6 \\
\hline
\end{tabular}

pendence of the fission-fragment yields upon excitation energy on the fission path. Note that the kinematical measurements of pre-neutron masses are subject to a finite resolution and uncertainties due to the correction for detector response and prompt-neutron emission.

b. Fission Induced by Thermal Neutrons: Mass distributions from thermal-neutron-induced fission are shown in Figs. 40 to 43

c. Fission Induced by Fast Neutrons: Fast-neutroninduced fission comprises the energy range of fission neutrons up to a few $\mathrm{MeV}$. Some data refer to well defined energies of e.g. $0.4 \mathrm{MeV}$ or $2 \mathrm{MeV}$, some correspond to rather broad energy distributions. In the case of a strongly energy-dependent fission cross section, the mean energy of the fissioning nuclei may be rather high. Therefore, in Figs. 44 to 47 the strongly energy-dependent yield at symmetry may often not be correctly reproduced by the calculations, which were performed with an incomingneutron energy of $2 \mathrm{MeV}$ in all cases. 
d. Fission Induced by 14-MeV Neutrons: A few mass distributions were measured for fission induced by 14-MeV neutrons. They are compared with results of the GEF code in Figs. 48 and 49

\section{Deviations}

The reduced Chi-squared values of the deviations between GEF results and evaluated data are given in Tables VIII and IX. There are no Chi-squared values given for distributions of pre-neutron or provisional masses because of several reasons. In some cases, the uncertainties are not available. Moreover, the data are disturbed by the finite mass resolution and possible problems in the corrections for prompt-neutron emission 33. The tables also give the references to the sources of the data shown in Figs. 36 to 49

\section{Charge Polarization and Emission of Prompt Neutrons}

The two fission products are not fully determined by their mass, because the protons and neutrons of the fissioning nucleus can be distributed in a different portion to the fragments at scission by charge polarization. Furthermore, the emission of prompt neutrons from the excited fragments tends to decrease the neutron excess of the fragments. For calculating the nuclide distributions after prompt-neutron emission for which the most precise data are available, both the charge polarization and the prompt-neutron emission must be considered. Data on the mass-dependent prompt-neutron multiplicity exist for a few fissioning systems. They provide the necessary information for disentangling the influence of charge polarization and prompt-neutron emission on the neutron excess of the fission products.

Fig. 50 shows the mean values and the standard deviations of the $Z$ distributions for fixed post-neutron mass. Available experimental data in the light fission-fragment group of four fissioning systems [81, 186, 189 191] are compared with the result of the GEF code. The agreement is generally very good, except for the heaviest nuclei of the system ${ }^{249} \mathrm{Cf}\left(\mathrm{n}_{\mathrm{th}}, \mathrm{f}\right)$. However, it is not clear, whether this discrepancy can be attributed to a shortcoming of the model, because the experiment suffered from insufficient $Z$ resolution in this range.

The mass-dependent mean prompt-neutron multiplicity for ${ }^{237} \mathrm{~Np}(\mathrm{n}, \mathrm{f})$ [80 and ${ }^{252} \mathrm{Cf}(\mathrm{sf})$ 192 are shown below in the dedicated section. Also here one can observe a good reproduction of the experimental data by the GEF code.

\section{Nuclide Distributions}

a. Thermal-neutron-induced Fission: A detailed overview on the nuclide distribution for thermal-neutron induced fission is shown in Figs. 51 to 56 for only one representative system because of the large quantity of this kind of data. In these figures, the empirical mass-chain yields for the system ${ }^{235} \mathrm{U}\left(\mathrm{n}_{\mathrm{th}}, \mathrm{f}\right)$ are compared with the result of the GEF code. In the light fission-product group, the data measured at the Lohengrin spectrograph have been chosen [81], while the evaluation of Wahl [3] was used for the heavy group. However, only those elements of this evaluation were taken for which at least two data points were directly derived from experimental
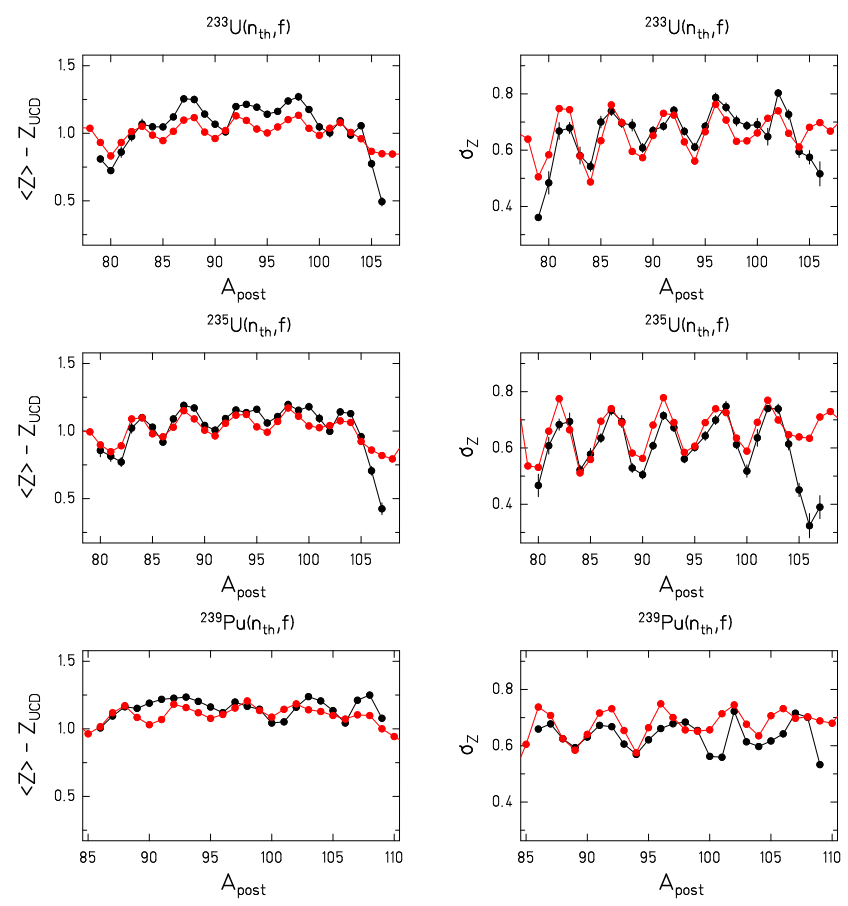

${ }^{239} \mathrm{Pu}\left(\mathrm{n}_{\text {th, }}, \mathrm{f}\right)$
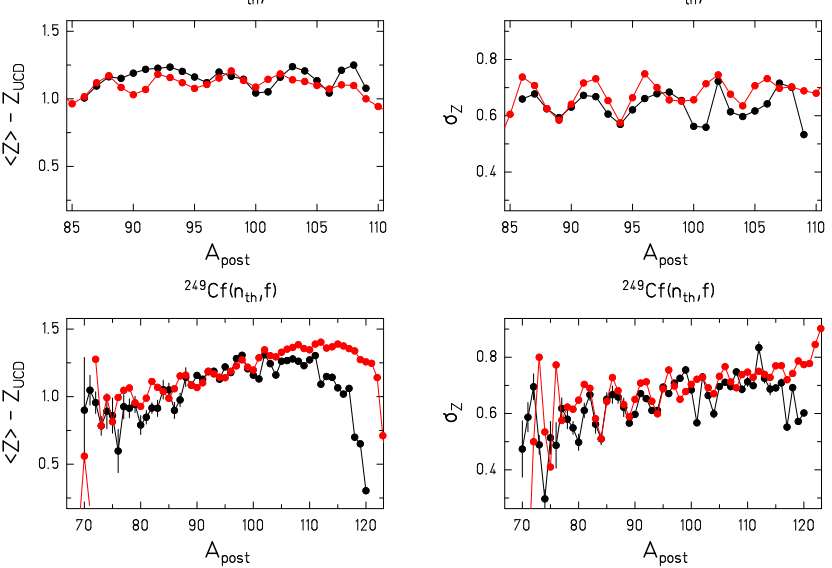

FIG. 50. (Color online) Charge density of post-neutron fragments. Deviation of the mean fission-product nuclear charge for a fixed mass after prompt-neutron emission from the UCD value (left column) and standard deviation of the isobaric $Z$ distribution (right column). Experimental data (black symbols) are compared with the results of the GEF code (red symbols). Error bars of the experimental data points are shown when they exceed the size of the symbols. The experimental uncertainties of the $\langle Z\rangle-Z_{U C D}$ values are typically below 0.02 charge units in the peak region of the mass distribution and around 0.05 charge units or more in the tails. The uncertainty in the range $A>110$ may be larger (see text). The experimental uncertainties of the $\sigma_{Z}$ values are typically around 0.01 charge units in the peak region of the mass distribution and around 0.04 charge units or more in the tails. 

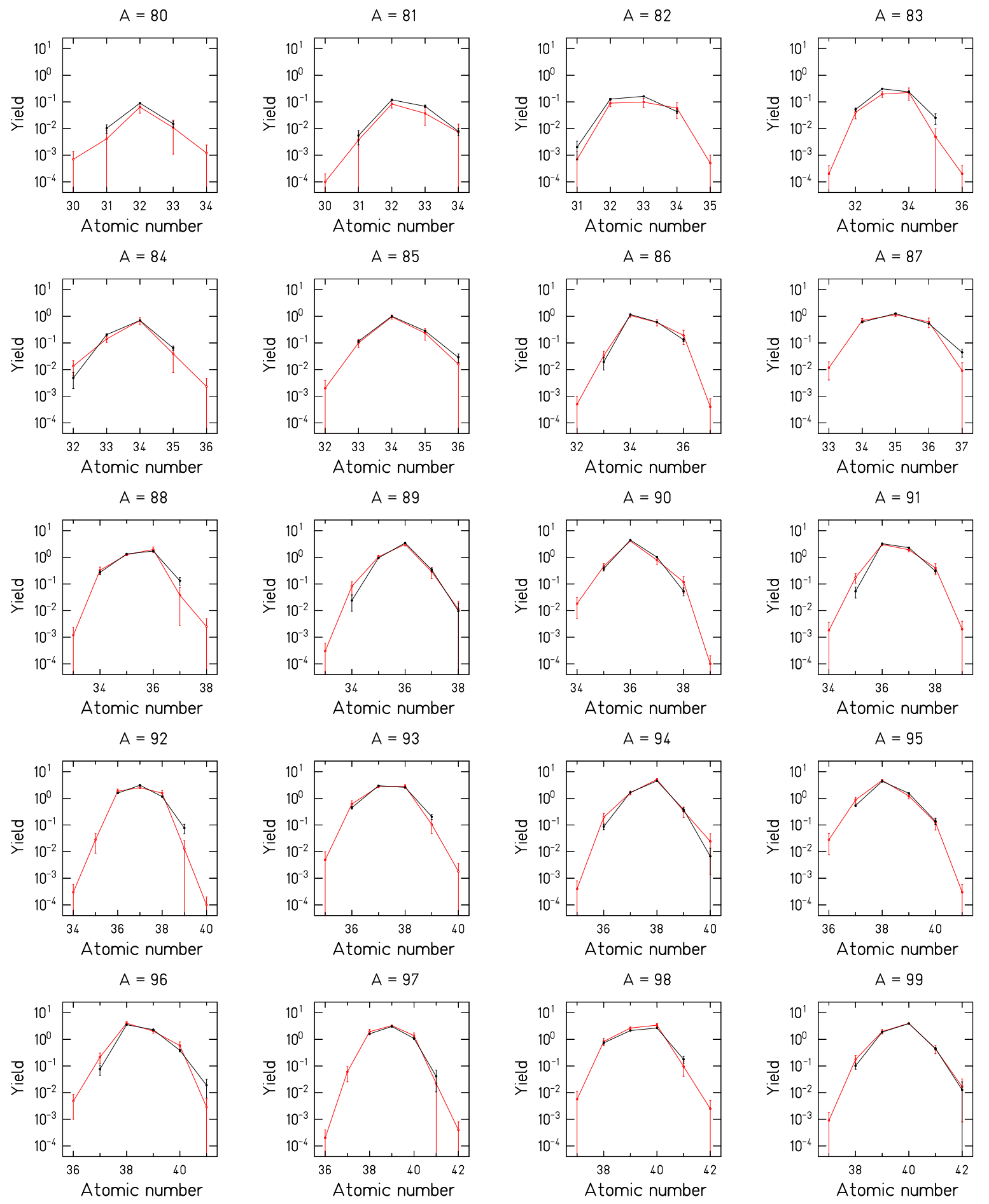

FIG. 51. (Color online) Post-neutron isobaric element distributions for ${ }^{235} \mathrm{U}\left(\mathrm{n}_{\mathrm{th}}, \mathrm{f}\right)$ in logarithmic scale, part 1. The experimental data 181] (black symbols) are compared with the results of the GEF code (red symbols). The mass numbers are specified in the figures. 

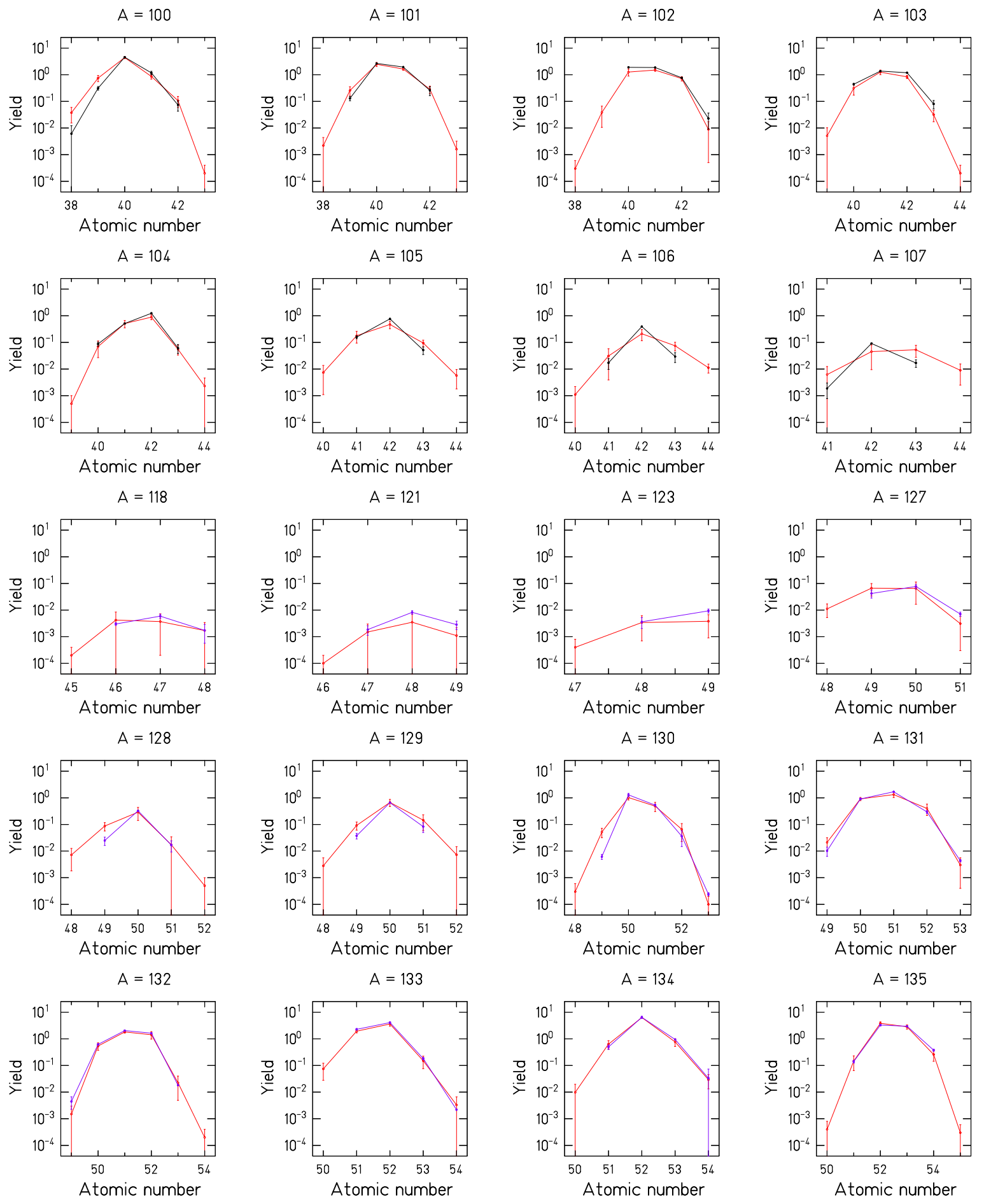

FIG. 52. (Color online) Post-neutron isobaric element distributions for ${ }^{235} \mathrm{U}\left(\mathrm{n}_{\mathrm{th}}, \mathrm{f}\right)$ in logarithmic scale, part 2. The experimental data [81] (black symbols) and the evaluated data [3] (violet symbols) are compared with the results of the GEF code (red symbols). The mass numbers are specified in the figures. 

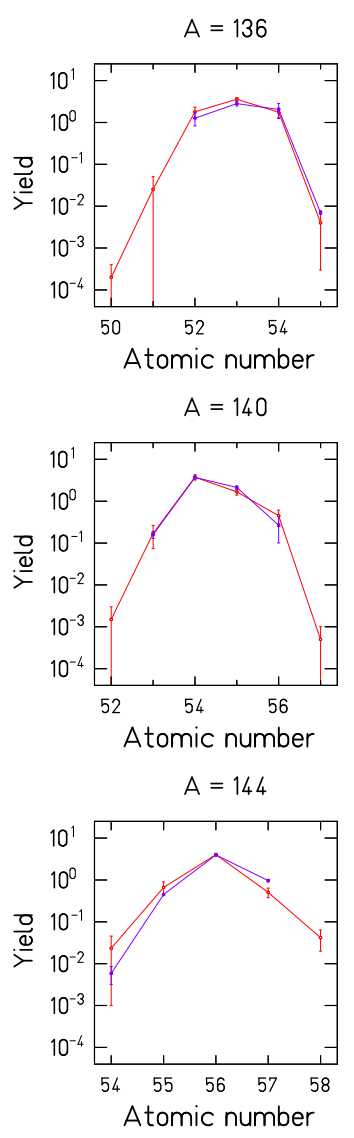
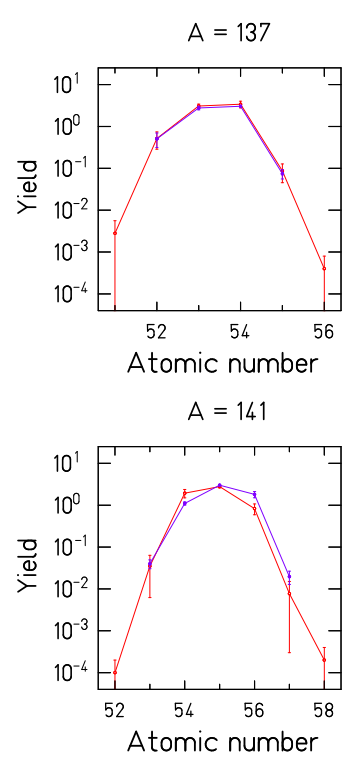

$A=145$

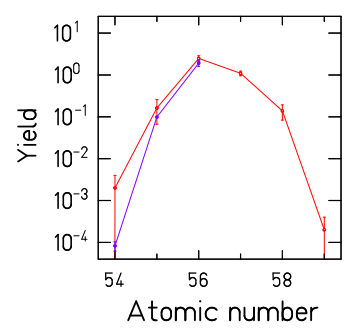

$A=138$

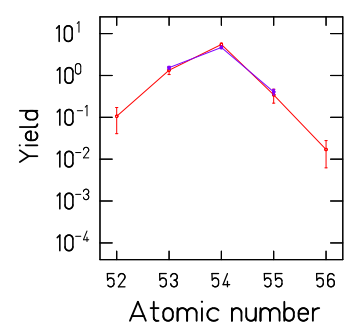

$A=142$

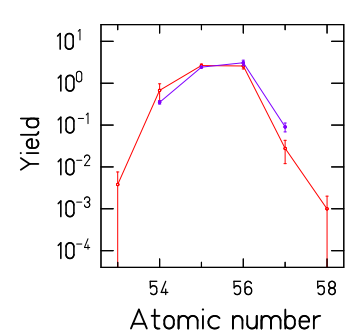

$A=146$

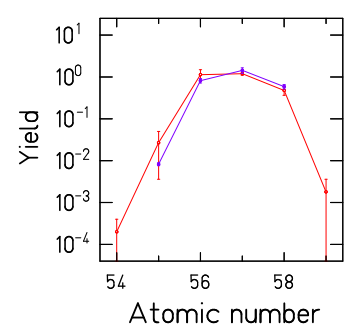

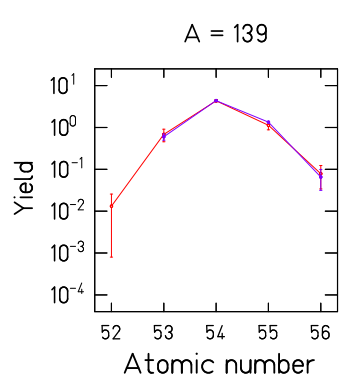

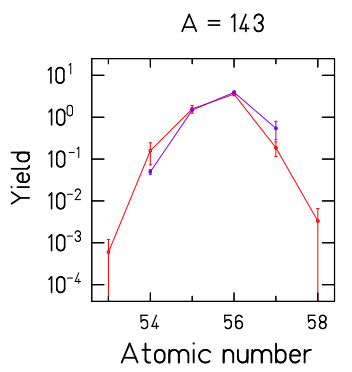

Atomic number

FIG. 53. (Color online) Post-neutron isobaric element distributions for ${ }^{235} \mathrm{U}\left(\mathrm{n}_{\mathrm{th}}, \mathrm{f}\right)$ in logarithmic scale, part 3 . The evaluated data [3] (violet symbols) are compared with the results of the GEF code (red symbols). The mass numbers are specified in the figures.

data.

In Figs. 51 to 53 , a logarithmic scale has been chosen, spanning the same range from $10^{-4 \%}$ to $10 \%$. The error bars of the empirical data represent the uncertainties given in [3, 81]. The error bars of the GEF results represent the estimated uncertainties that were obtained by calculations with perturbed parameters. The relevant parameters were varied within their uncertainty range.

In Figs. 54 to 56, the same data are shown in a linear scale. The range is adjusted to the range of the data in each case individually.

b. Fission after Heavy-ion Fusion: Very recently, measured nuclide distributions from fission of ${ }^{250} \mathrm{Cf}$ at an excitation energy as high as $45 \mathrm{MeV}$ became available in an inverse-kinematics experiment, using a ${ }^{238} \mathrm{U}$ primary beam with an energy of $6.1 \mathrm{~A} \mathrm{MeV}$ [174, impinging on a ${ }^{12} \mathrm{C}$ target. This is a large step in energy from the experiments with full identification of the fission products in $A$ and $Z$ using kinematical methods that were performed previously with thermal-neutron-induced fission in normal kinematics (see Sec. IX B 2). Fig. 57 shows a comparison of the isotopic mass yields with the result of the GEF code. This comparison is a stringent test of the reliability of the GEF code for the application to fission from high excitation energies.

Fig. 57 reveals that the measured nuclide yields are rather well reproduced by the GEF code over the whole fission-product range that was fully covered in the experiment. In particular, the peak position is almost perfectly reproduced, expect for the heaviest elements, where a slight shift with respect to the experiment is observed. The widths of the isotopic mass distributions are slightly underestimated by the GEF model. Further studies are needed to determine, whether this is a sign for a temperature-induced broadening of the charge polarization or for a slightly increased width of the promptneutron multiplicity distribution that could result from stronger shape fluctuations at scission.

The mass distribution shown in Fig. 58 deviates clearly from a Gaussian shape. The flat top indicates that even for this highly excited nucleus, the fission process is still influenced by shell structure. The result of the GEF model reproduces this feature nicely. According to the model, the flat top is caused by fission from lower excitation energies after the evaporation of several neutrons. The deviation above $A=150$ is due to a known kinemat- 

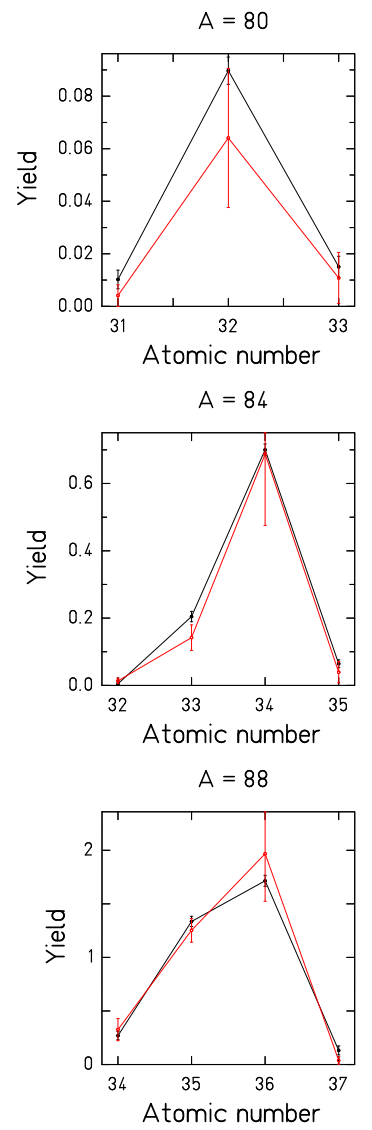

$A=92$
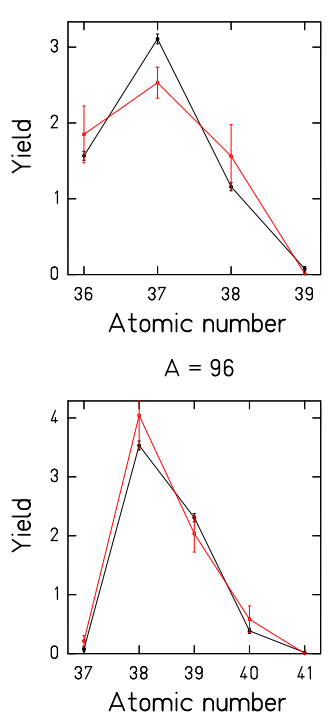

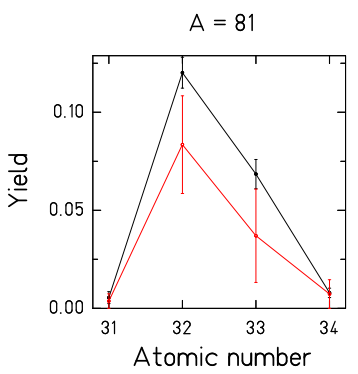

$A=85$
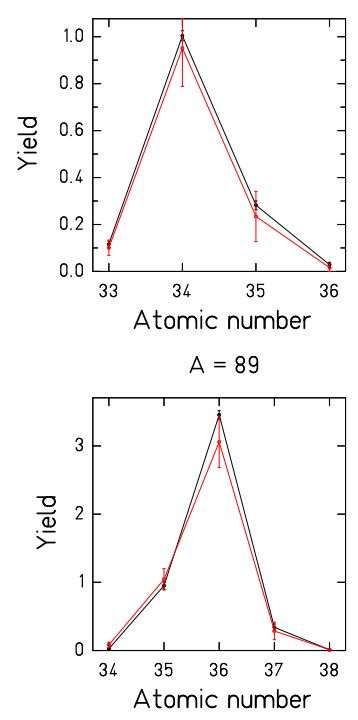

$$
A=93
$$
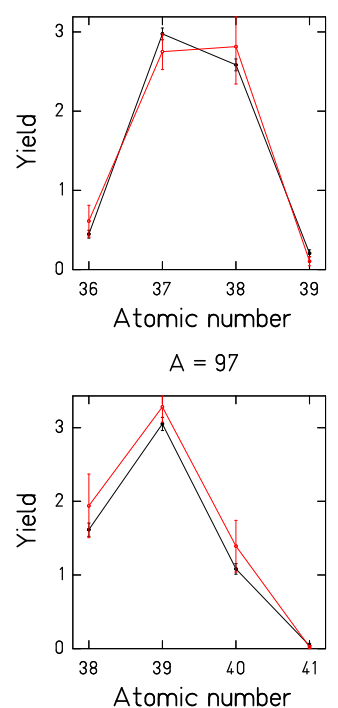

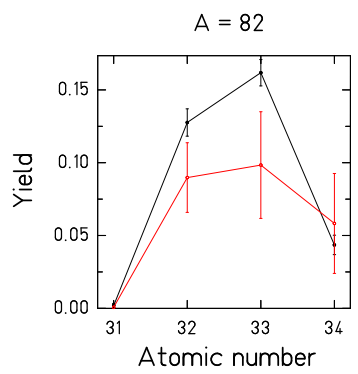

$$
A=86
$$
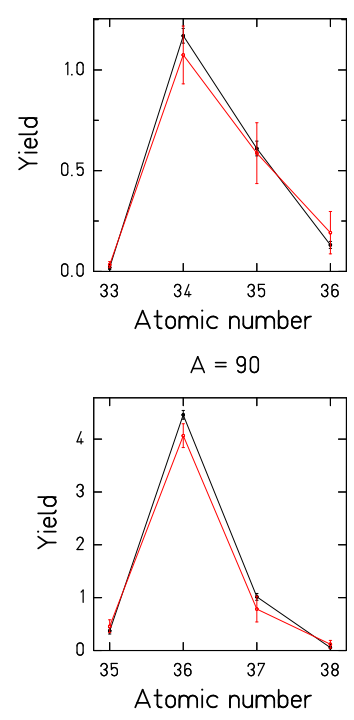

$A=94$
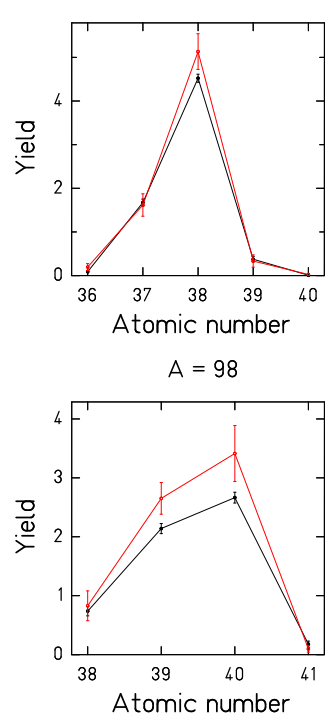

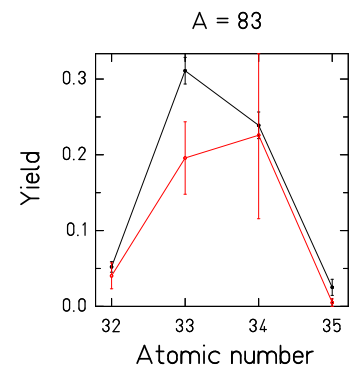

$A=87$
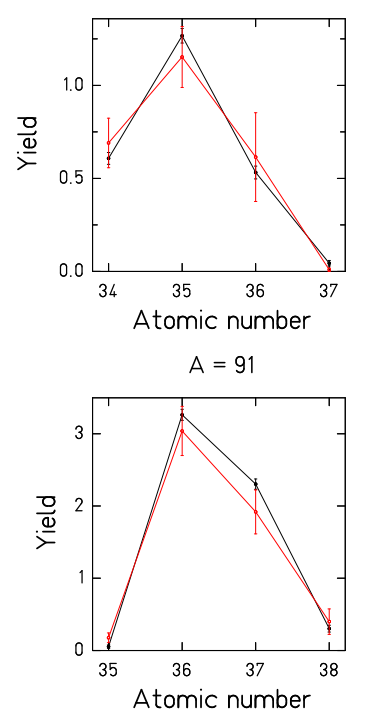

$A=95$

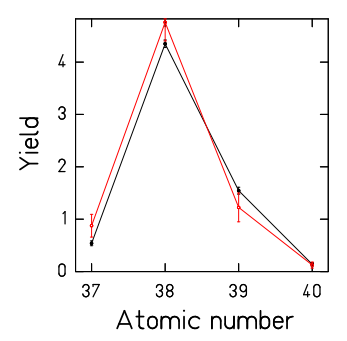

$A=99$

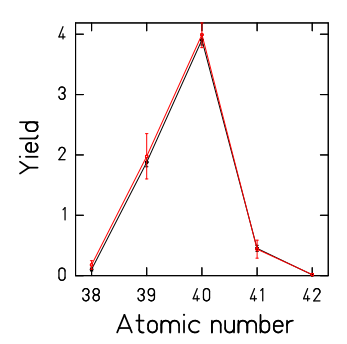

FIG. 54. (Color online) Post-neutron isobaric element distributions for ${ }^{235} \mathrm{U}\left(\mathrm{n}_{\mathrm{th}}, \mathrm{f}\right)$ in linear scale, part 1 . The experimental data [81] (black symbols) are compared with the results of the GEF code (red symbols). The mass numbers are specified in the figures. 

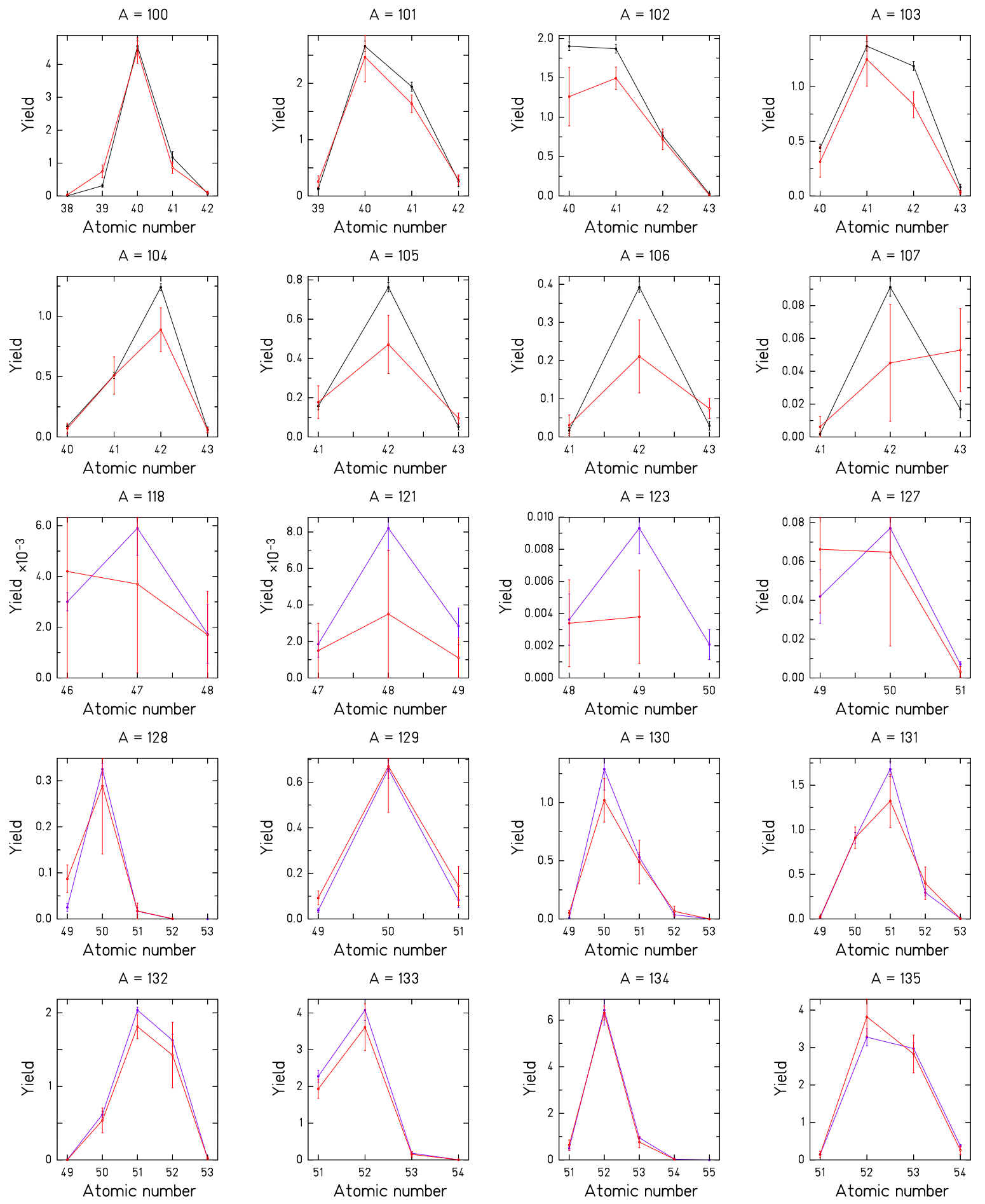

FIG. 55. (Color online) Post-neutron isobaric element distributions for ${ }^{235} \mathrm{U}\left(\mathrm{n}_{\mathrm{th}}, \mathrm{f}\right)$ in linear scale, part 2. The experimental data [81] (black symbols) and the evaluated data [3] (violet symbols) are compared with the results of the GEF code (red symbols). The mass numbers are specified in the figures. 

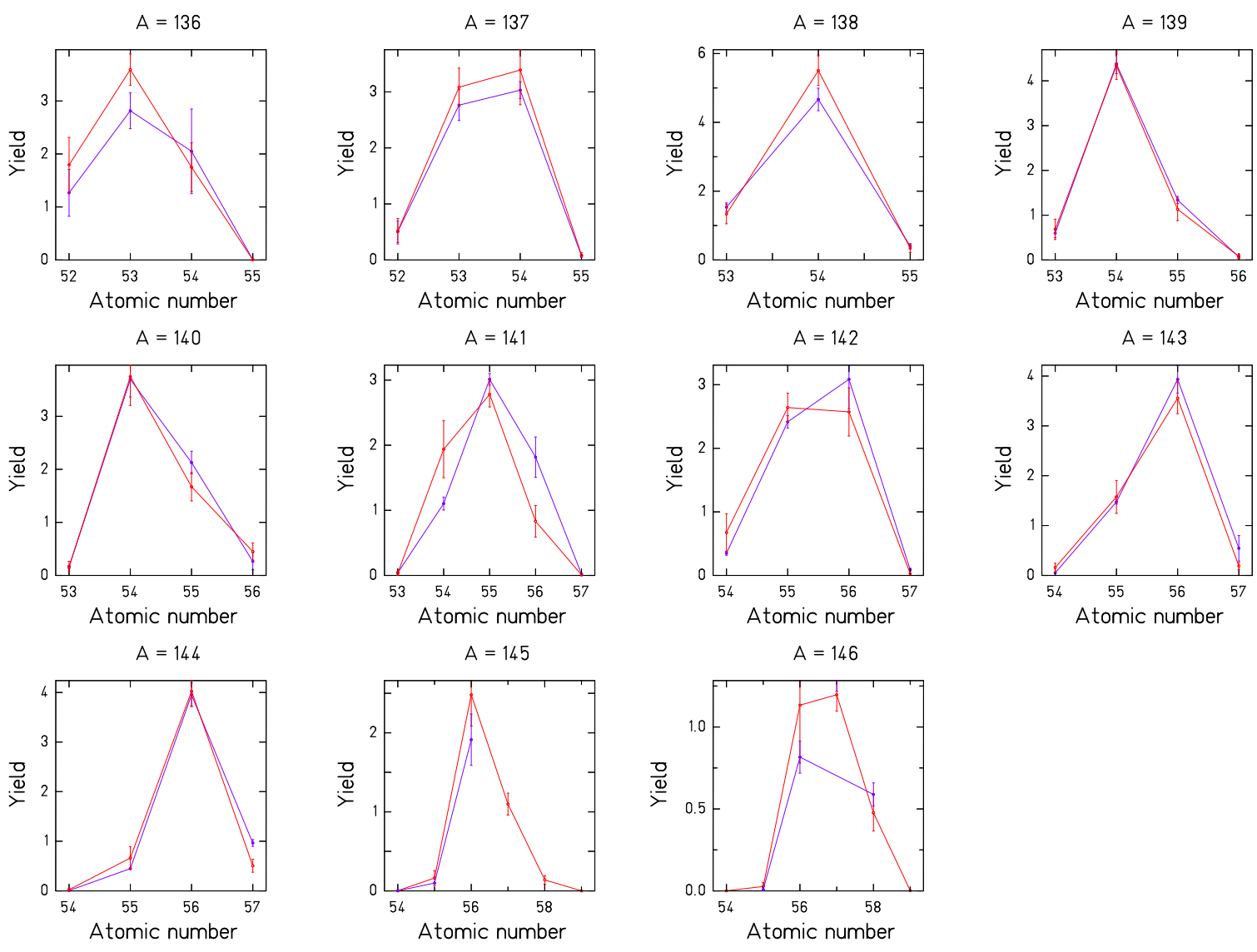

FIG. 56. (Color online) Post-neutron isobaric element distributions for ${ }^{235} \mathrm{U}\left(\mathrm{n}_{\mathrm{th}}, \mathrm{f}\right)$ in linear scale, part 3. The evaluated data 3$]$ (violet symbols) are compared with the results of the GEF code (red symbols). The mass numbers are specified in the figures.

TABLE X. Calculated most probable fission energies and probabilities of the different fission chances for fission of ${ }^{250} \mathrm{Cf}$ at $E^{*}=45 \mathrm{MeV}$ and $J_{r m s}=20 \hbar$.

\begin{tabular}{c|c|c}
\hline Chance & $E_{\text {peak }}^{*}(\mathrm{MeV})$ & Probability \\
\hline 1. & 45.0 & $39.0 \%$ \\
2. & 37.3 & $31.8 \%$ \\
3. & 29.5 & $22.2 \%$ \\
4. & 20.9 & $6.4 \%$ \\
5. & 14.2 & $0.5 \%$ \\
\hline \hline
\end{tabular}

ical cut in the experiment.

The calculated contributions of the different fission chances are listed in Table X. In addition, Fig. 59 demonstrates that in first-chance fission shell effects have completely disappeared, and a Gaussian-type mass distribution is obtained. Fission after evaporation of one or several neutrons shows more and more features of the double-humped distribution that is typical for low-energy fission of the actinides and finally produces the observed mass distribution with the flat top.

\section{Energy Dependence}

In order to benchmark the GEF code up to $14 \mathrm{MeV}$, the fission yields of 3 masses are compared with the available data. As suggested in Ref. [193], the masses 111, 115 and 140 were chosen. In order to be comparable, the shell effect at symmetry was set to $+0.3 \mathrm{MeV}$ for all systems, corresponding to a weak anti-shell. First, the ratio $Y(A=115) / Y(A=140)$ is shown in Fig. 60. The GEF code is able to reproduce the global trend for all systems. Also the absolute values agree very well for the systems ${ }^{238} \mathrm{U}$ and ${ }^{239} \mathrm{Pu}$, while they are slightly overestimated for ${ }^{235} \mathrm{U}$ and ${ }^{232} \mathrm{Th}$. Thus, the conditional barrier for symmetric fission seems to be slightly higher for these two systems.

Two regions in energy domain should be studied: before the second-chance threshold, where the fissioning nucleus is always the same but with different excitation energy and after this threshold, where multi-chance fission must be taken into account. While Fig. 60 shows the evolution of the valley/peak ratio, represented by the $A=115 / A=140$ ratio, before the threshold $\left(E^{*} \approx 10\right.$ $12 \mathrm{MeV}$ ) for neutron-induced fission of ${ }^{232} \mathrm{Th},{ }^{235} \mathrm{U},{ }^{238} \mathrm{U}$, 

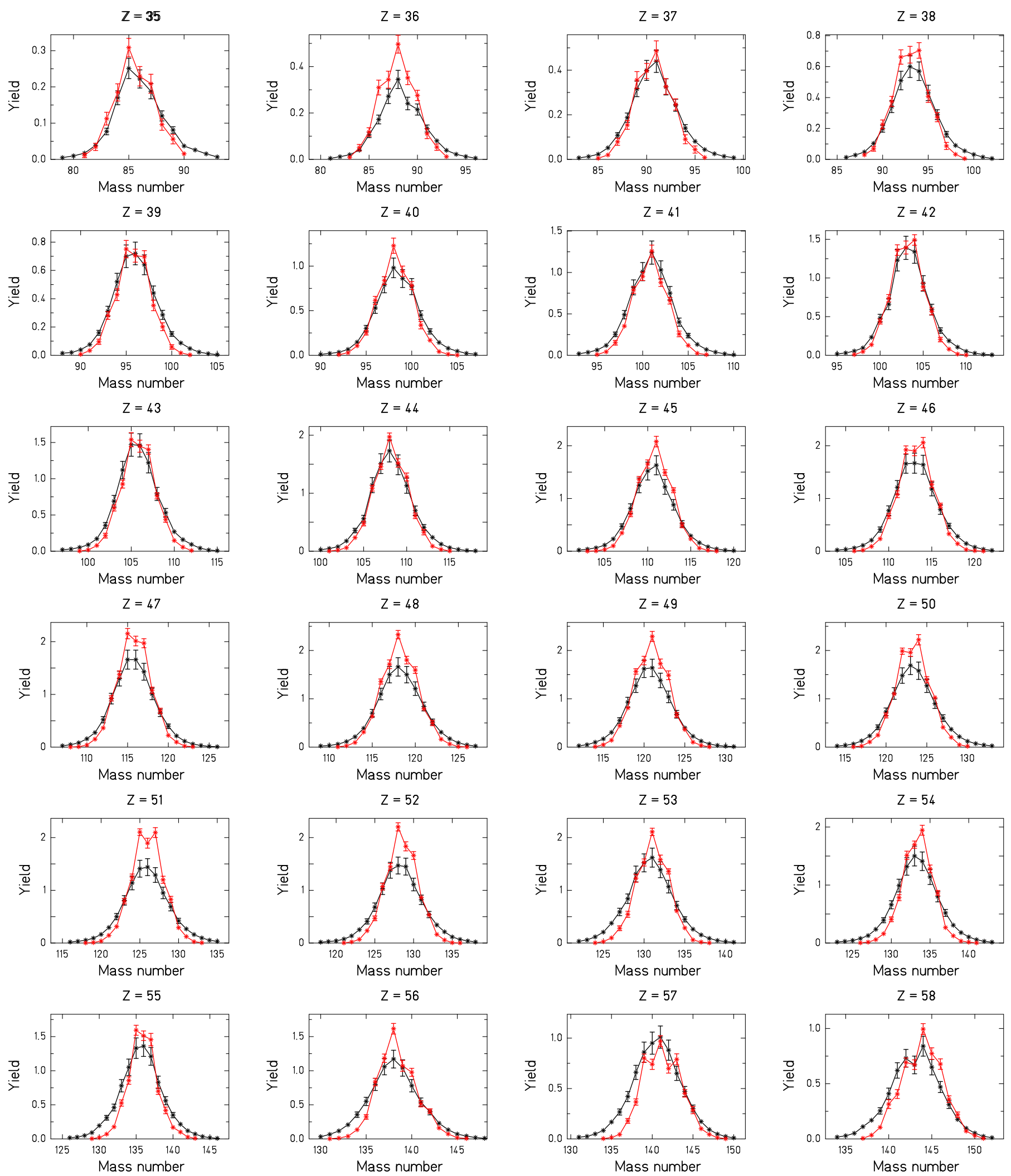

FIG. 57. (Color online) Post-neutron isotopic $A$ distributions for the fission of ${ }^{250} \mathrm{Cf}$ at an excitation energy $E^{*}=45 \mathrm{MeV}$ and an rms angular momentum of $20 \hbar$. The experimental data [174] (black symbols) are compared with the results of the GEF code (red symbols). The elements are specified in the figures. Note the different scales! 


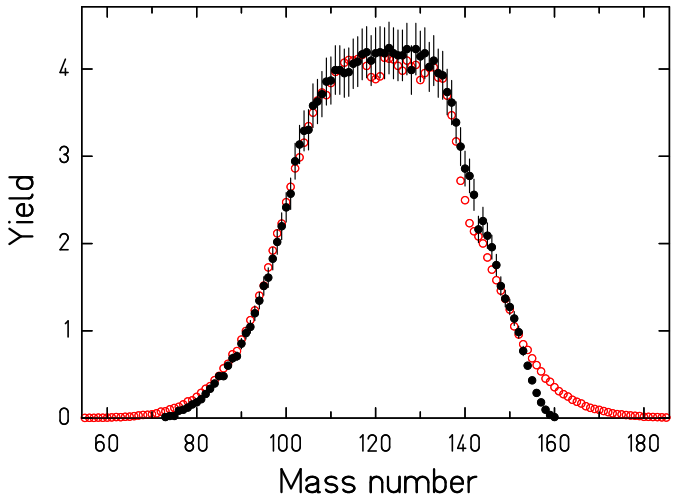

FIG. 58. (Color online) Mass distribution for the fission of ${ }^{250} \mathrm{Cf}$ at an excitation energy $E^{*}=45 \mathrm{MeV}$ and an rms angular momentum of $20 \hbar$. The experimental data 174] (black full symbols) are compared with the results of the GEF code (red open symbols).

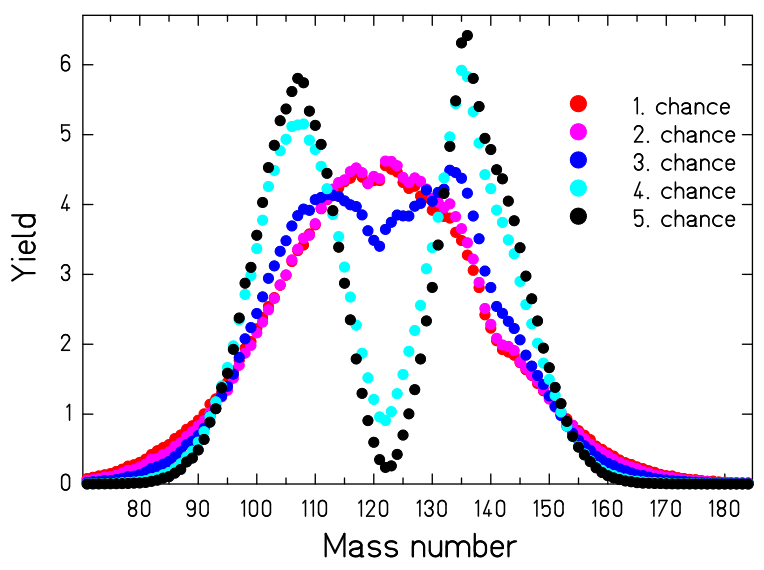

FIG. 59. (Color online) Calculated mass distribution for the most probable excitation energies (see Table X) of the different fission chances of ${ }^{250} \mathrm{Cf}$ at an initial excitation energy $E^{*}=$ $45 \mathrm{MeV}$ and an rms angular momentum of $20 \hbar$.

and ${ }^{239} \mathrm{Pu}$, the fission yields of two masses near symmetry ( $A=111, A=115)$ and one mass near the heavy peak ( $A$ $=140)$ are compared in Figs. 61 and 62 for the systems ${ }^{235} \mathrm{U}$ and ${ }^{239} \mathrm{Pu}$ in an extended energy range. Obviously, the values above the threshold for second-chance fission $\left(E_{n} \approx 6 \mathrm{MeV}\right)$ and third-chance fission $\left(E_{n} \approx 12 \mathrm{MeV}\right)$ are well reproduced. The calculation slightly underestimates the yields of the system ${ }^{235} \mathrm{U}$ near symmetry above the threshold of second-chance fission, a deviation which is opposite to the deviation found at lower energies revealed in Fig. 60. However, the yield near the asymmetric peak at $A=140$ is underestimated in this low-energy range for both systems.

According to Tables XI and XII, the calculated proba-

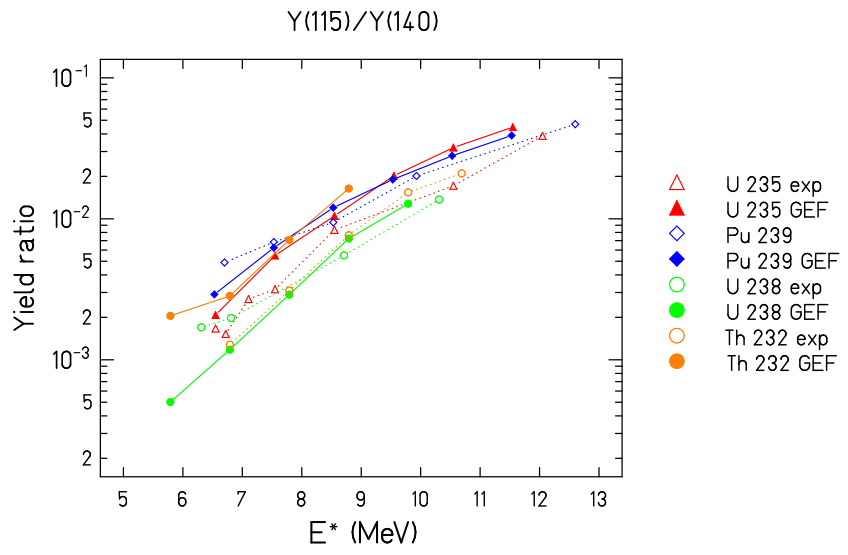

FIG. 60. (Color online) $Y(A=115) / Y(A=140)$ for different fissioning nuclei as a function of the excitation energy. Experimental data [194-197] are compared with the result of the GEF code.

bilities for first-chance fission at $E_{n}=8 \mathrm{MeV}$ and $E_{n}=$ $14 \mathrm{MeV}$ are somewhat higher, but still close to the values given in the ENDF/B-VII library [179].

TABLE XI. First-chance probability for ${ }^{235} \mathrm{U}(\mathrm{n}, \mathrm{f}), E_{n}=8$ $\mathrm{MeV}$ and $14 \mathrm{MeV}$.

\begin{tabular}{c|c|c}
\hline \hline Energy & $\begin{array}{c}\text { GEF first-chance } \\
\text { relative probability }\end{array}$ & $\begin{array}{c}\text { Library first-chance } \\
\text { relative probability }\end{array}$ \\
\hline $8 \mathrm{MeV}$ & $54.3 \%$ & $46.1 \%$ \\
$14 \mathrm{MeV}$ & $25.2 \%$ & $29.6 \%$ \\
\hline \hline
\end{tabular}

TABLE XII. First-chance probability for ${ }^{239} \mathrm{Pu}(\mathrm{n}, \mathrm{f}), E_{n}=8$ $\mathrm{MeV}$ and $14 \mathrm{MeV}$.

\begin{tabular}{c|c|c}
\hline \hline Energy & $\begin{array}{c}\text { GEF first-chance } \\
\text { relative probability }\end{array}$ & $\begin{array}{c}\text { Library first-chance } \\
\text { relative probability }\end{array}$ \\
\hline $8 \mathrm{MeV}$ & $80.4 \%$ & $65.6 \%$ \\
$14 \mathrm{MeV}$ & $59.5 \%$ & $44.8 \%$ \\
\hline \hline
\end{tabular}

As the difference of some specific masses can be the result of some local effects, the complete fission-yield distributions were studied for $E_{n}=4 \mathrm{MeV}, 8 \mathrm{MeV}$ and $14 \mathrm{MeV}$ where library evaluations are also available. Fig. 63 shows the fission-yield distributions for ${ }^{235} \mathrm{U}$ at $E_{n}=4$ and 8 $\mathrm{MeV}$. The predicted fission-yield distribution at $E_{n}=$ $4 \mathrm{MeV}$ overestimates the experimental data slightly. At $E_{n}=8 \mathrm{MeV}$, there exist two data-sets from different experiments. The GEF prediction is close to the symmetric data of Glendenin et al. 194 but higher than the data from Chapman et al. 201]. This is in line with an analysis reported in 193], where comparisons were made with the Ford experimental data 198] which are consis- 
Fission Yields $A=140$

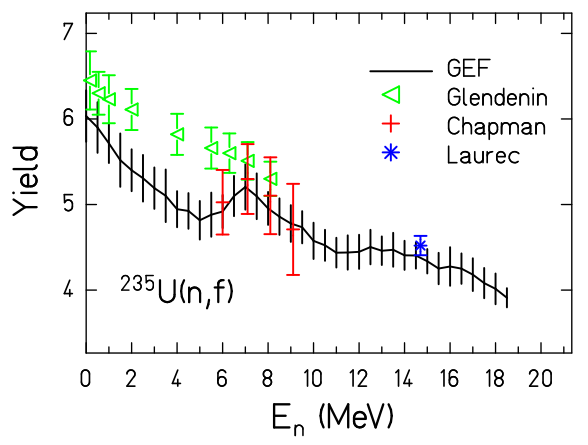

Fission Yields $A=115$

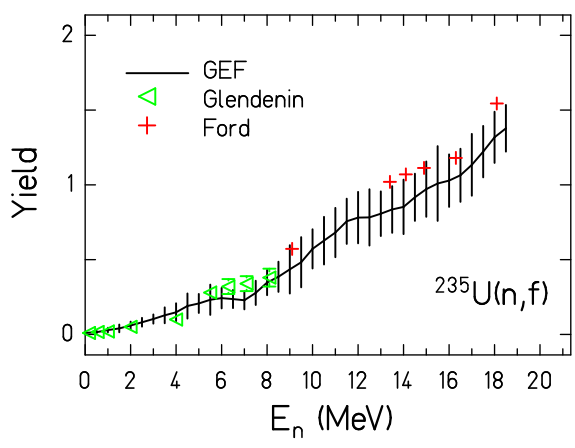

Fission Yields $A=111$

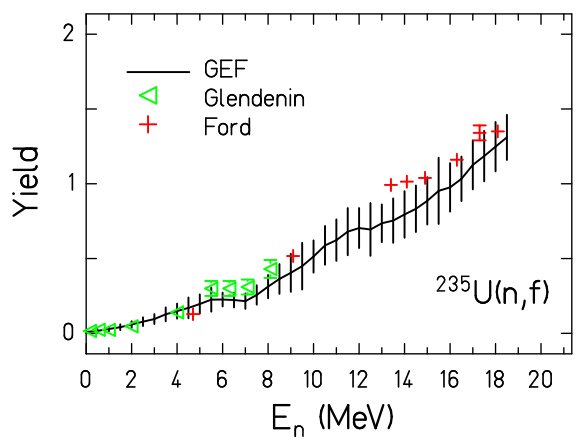

FIG. 61. (Color online) Measured fission yields of $A=140$ (top), $A=115$ (middle) and $A=111$ (bottom) for ${ }^{235} \mathrm{U}(\mathrm{n}, \mathrm{f})$ as a function of $E_{n}$ [194, 198, 199] are compared with the GEF results. The hatched band indicates the uncertainty of the calculated values.

tent with the Glendenin data, according to which the Chapman data seem to systematically underestimate the symmetric part.

The fission yields of the system ${ }^{239} \mathrm{Pu}(\mathrm{n}, \mathrm{f})$ at $E_{n}=4$ and $8 \mathrm{MeV}$ shown in Fig. 64 are rather well reproduced by the GEF code.

In addition to the comparison at the lower energies $\left(E_{n}=4\right.$ and $\left.8 \mathrm{MeV}\right)$, the fission yields of ${ }^{235} \mathrm{U}$ and ${ }^{239} \mathrm{Pu}$ at $E_{n}=14 \mathrm{MeV}$ are shown in Fig. 65. Some deviations to the evaluated data are found: The calculated fission yields at symmetry are slightly lower than the eval-
Fission Yields $A=140$

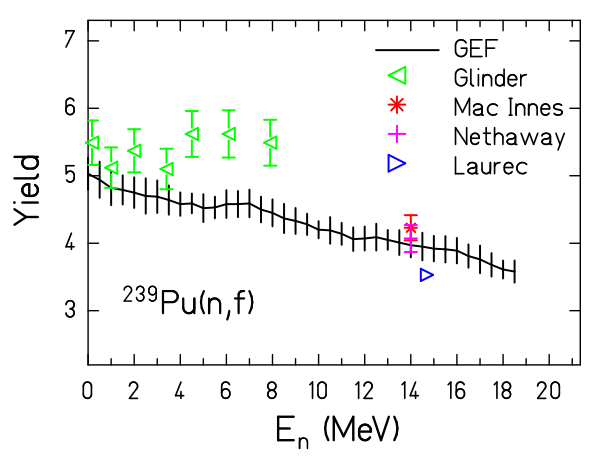

Fission Yields $A=115$

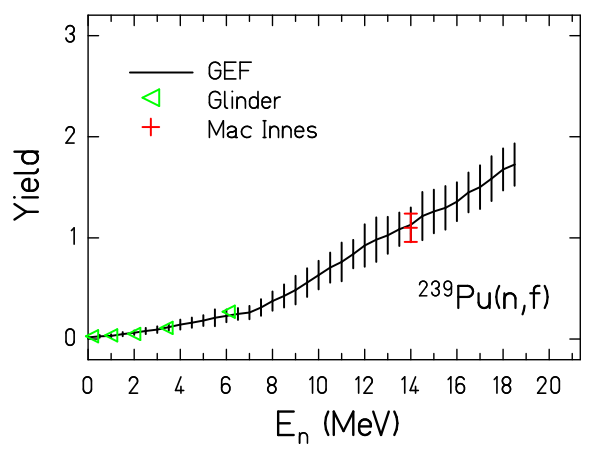

Fission Yields $A=111$

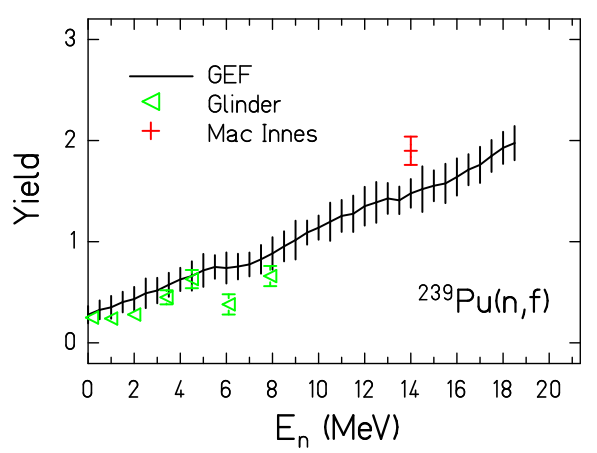

FIG. 62. (Color online) Measured fission yields of $A=140$ (top), $A=115$ (middle) and $A=111$ (bottom) for ${ }^{239} \mathrm{Pu}(\mathrm{n}, \mathrm{f})$ as a function of $E_{n}$ [195, 199, 200] are compared with the GEF results. The hatched band indicates the uncertainty of the calculated values.

uated values. However, in view of the scattering of the data from different experiments this deviation is not very significant. Moreover, the calculated yield distribution shows a more pronounced structure in the peak regions if compared to the evaluation. Also, this deviation is not very significant due to the uncertainties and the large scattering of the experimental data. 


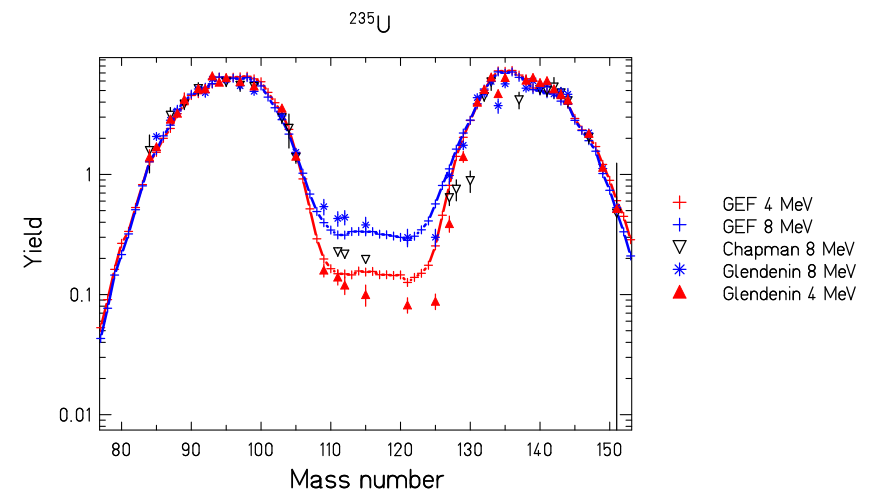

FIG. 63. (Color online) Fission-yield distribution for ${ }^{235} \mathrm{U}(\mathrm{n}, \mathrm{f})$ for $E_{n}=4 \mathrm{MeV}$ and $E_{n}=8 \mathrm{MeV}$. Experimental data 194, 201] are compared with the GEF results.

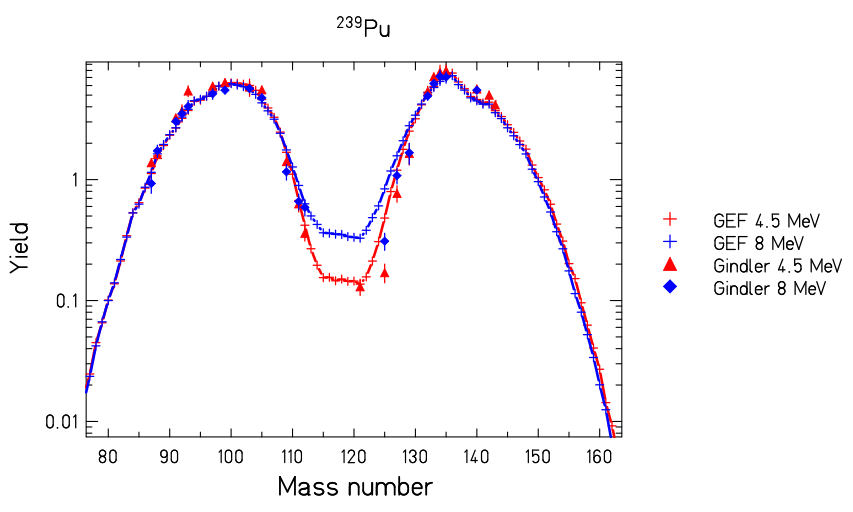

FIG. 64. (Color online) Fission-yield distribution for ${ }^{239} \mathrm{Pu}(\mathrm{n}, \mathrm{f})$ for $E_{n}=4 \mathrm{MeV}$ and $E_{n}=8 \mathrm{MeV}$. Experimental data [195] are compared with the GEF results.

\section{Discussion}

a. Mass Distributions: Figs. 36 to 49 demonstrate an overall rather good agreement between the empirical mass distributions and the results of the GEF code. In particular, the variation of the global shape for different systems and as a function of energy, which can be rather drastic in some cases, are rather well reproduced. When looking in detail, however, more or less severe discrepancies can also be found for several systems. In this comparison, it must be considered that the quality of the experimental data that are shown in some figures or that were used for the evaluation may differ strongly from one system to another one. In many cases, the evaluation is based on incomplete data of limited quality due to the difficulties of the experiment. In other cases, there are plenty of high-quality data. The mass distributions from double-energy or double-velocity measurements generally suffer from a limited resolution. The data from spontaneous fission of the heaviest nuclei are important for
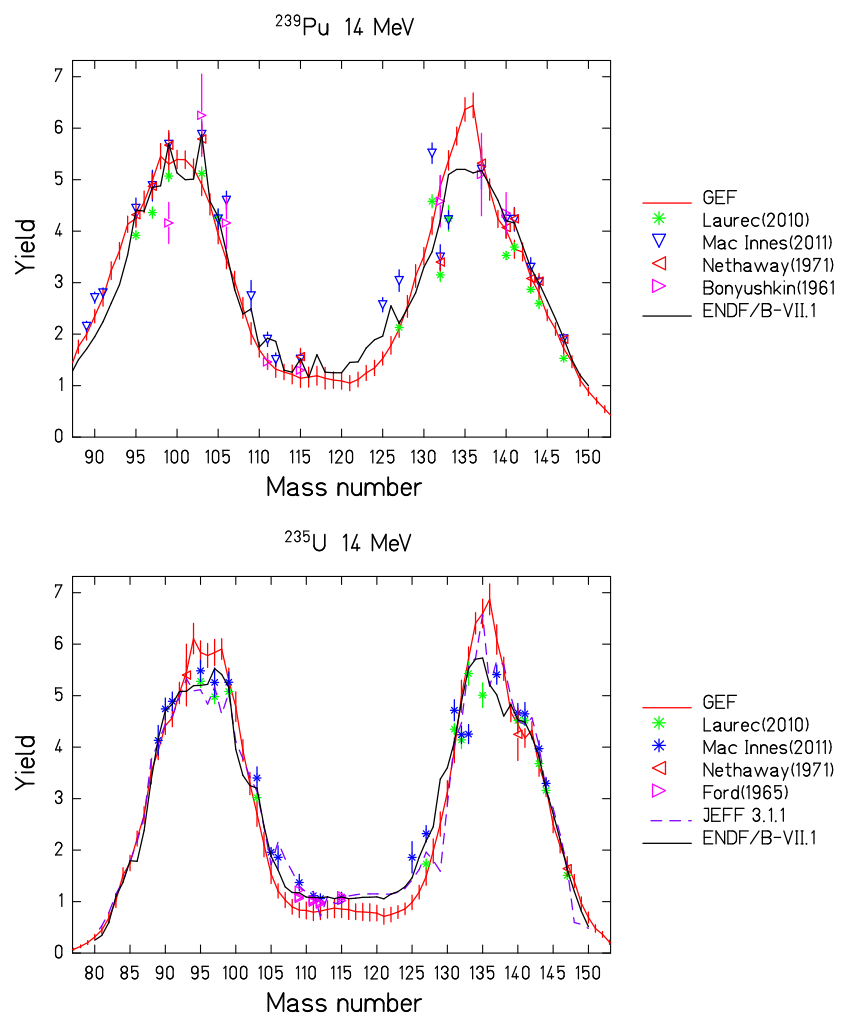

FIG. 65. (Color online) Fission-yield mass distributions for ${ }^{235} \mathrm{U}(\mathrm{n}, \mathrm{f})$ and ${ }^{239} \mathrm{Pu}(\mathrm{n}, \mathrm{f})$ at $E_{n}=14 \mathrm{MeV}$. The result of the GEF model is compared with values from data libraries and experimental data. Color points correspond to experimental data [198 200]. The calculated values are given together with their estimated uncertainties

revealing the strong variation of the global shape from system to system, but the uncertainties are rather large, e.g. due to low statistics and the limited mass resolution. Thus, a careful analysis is needed to decide whether the discrepancies between empirical data and calculated spectra are to be attributed to shortcomings of the model or to uncertainties of the empirical data.

A first step towards a quantitative assessment is the determination of the reduced Chi-squared values of the differences between empirical data and calculated values. The Chi-squared values were only determined for the evaluated data, because they are mostly based on radiochemical methods with full identification of the fissionproduct mass, while the mass spectra from kinematical measurements are distorted by the limited resolution. These Chi-squared values are listed in Table VIII.

The Chi-squared values are also shown in an histogram in Fig. 66. The distribution has a main peak around unity, containing 50 of the 59 cases. It reaches from 0.3 to 1.7 and, thus, seems to be essentially in agreement with the expected scattering caused by the uncertainties of the evaluated data. This picture already does not give indications for a shortcoming of the model in these 50 
cases which represent $85 \%$ of the cases. The uncertainties of the model seem to contribute little to the Chi-squared values of the systems in the main peak, because this peak centers at about unity without taking the uncertainties of the model into account. The remaining 9 cases will be investigated in more detail.

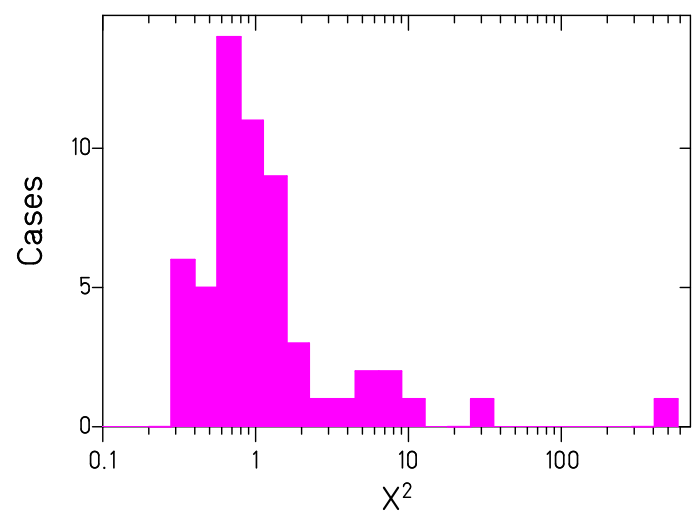

FIG. 66. (Color online) Chi-squared deviations of the mass distributions from GEF calculations from the empirical data (Figs. 36 to 49) in a logarithmic binning. The height of the histogram represents the number of cases per bin.

The largest Chi-squared value is observed for the system ${ }^{227} \operatorname{Th}\left(\mathrm{n}_{\mathrm{th}}, \mathrm{f}\right)$. An inspection of the figure, in particular in the logarithmic scale, reveals that the evaluated spectrum has a very unusual shape that differs substantially from the other spectra of near-by systems: The descent from the asymmetric mass peaks towards symmetry is exceptionally gradual. This observation is a strong argument for assuming that the problem is caused by an unrealistic result of the evaluation in this case.

The second-highest Chi-squared value is found for the system ${ }^{229} \mathrm{Th}\left(\mathrm{n}_{\mathrm{th}}, \mathrm{f}\right)$. Also in this case, the largest deviations occur on the inner wings of the asymmetric peaks. This time, the slope agrees, but the borders towards symmetry are shifted in the calculation. In this case, there exist very reliable and accurate data from different sources, including an experiment at the Lohengrin spectrograph [186]. Thus, this problem must be attributed to a shortcoming of the model. This displacement of the inner wing of the asymmetric mass peak with respect to the global description of the model, which agrees in practically all other cases, is very astonishing. It indicates a local effect that is not considered in the model.

This system is particular in two aspects: Firstly, the potential-energy gain from saddle to scission is especially low 94] compared to the other systems included in this study. Thus, the excitation energy along the fission path is lower than for most of the other systems. Secondly, there are indications that the fission barriers of the nuclei in this region have a complex structure with several consecutive saddle points [187, 188], whereby the height of the third barrier, relative to the inner saddles, grows towards lighter nuclei. Considering that the system ${ }^{238} \mathrm{U}(\mathrm{sf})$ that probably has an even lower excitation energy along the fission path than ${ }^{229} \mathrm{Th}\left(\mathrm{n}_{\mathrm{th}}, \mathrm{f}\right)$ does not show the anomaly in the inner wings of the mass distribution, it seems tempting to attribute this anomaly to the influence of the third barrier on the evolution of the mass-asymmetry degree of freedom. Maybe, the most symmetric mass splits of the asymmetric component are hindered by the potential-energy landscape around the third barrier. This may be linked to the fact that these are the most compact configurations that are characterized by a large Coulomb energy. More data on fully identified fission products for systems in this region that will be provided by the SOFIA experiment [36] will help to get more insight into this problem. Guidance is also expected from dedicated studies with microscopic models.

Seven other systems show larger Chi-squared values between 3.2 and 9.5: ${ }^{238} \mathrm{U}(\mathrm{sf}),{ }^{248} \mathrm{Cm}(\mathrm{sf}),{ }^{253} \mathrm{Es}(\mathrm{sf})$, ${ }^{235} \mathrm{U}\left(\mathrm{n}_{\mathrm{th}}, \mathrm{f}\right),{ }^{251} \mathrm{Cf}\left(\mathrm{n}_{\mathrm{th}}, \mathrm{f}\right),{ }^{254} \operatorname{Es}\left(\mathrm{n}_{\mathrm{th}}, \mathrm{f}\right)$, and ${ }^{255} \mathrm{Fm}\left(\mathrm{n}_{\mathrm{th}}, \mathrm{f}\right)$. The deviations for ${ }^{238} \mathrm{U}(\mathrm{sf})$ are not severe and look unsystematical. $\quad{ }^{248} \mathrm{Cm}(\mathrm{sf}), \quad{ }^{253} \mathrm{Es}(\mathrm{sf}), \quad{ }^{251} \mathrm{Cf}\left(\mathrm{n}_{\mathrm{th}}, \mathrm{f}\right)$, ${ }^{254} \operatorname{Es}\left(\mathrm{n}_{\mathrm{th}}, \mathrm{f}\right)$, and ${ }^{255} \mathrm{Fm}\left(\mathrm{n}_{\mathrm{th}}, \mathrm{f}\right)$ form a group of nuclei that seem to suffer from incomplete data and/or large uncertainties. A closer look reveals two abnormalities: All systems show rather schematic shapes at the outer wings of the mass distributions that differ substantially from the spectrum of ${ }^{252} \mathrm{Cf}(\mathrm{sf})$ which has been investigated in great detail. In addition, ${ }^{254} \operatorname{Es}\left(\mathrm{n}_{\mathrm{th}}, \mathrm{f}\right)$ and even more clearly ${ }^{255} \mathrm{Fm}\left(\mathrm{n}_{\mathrm{th}}, \mathrm{f}\right)$ show a shift of the minimum around symmetry with respect to the calculation. The mass distribution of ${ }^{255} \mathrm{Fm}\left(\mathrm{n}_{\mathrm{th}}, \mathrm{f}\right)$ is symmetric around $A=128 \pm 0.5$, which is half the mass of the fissioning system. Thus, there is no room for neutron evaporation, although the systematics suggests a mean promptneutron yield around 5. Finally, ${ }^{235} \mathrm{U}\left(\mathrm{n}_{\mathrm{th}}, \mathrm{f}\right)$ is a very peculiar case. For this nucleus, the measurements are so precise that the experimental uncertainties are appreciably smaller than the general uncertainties of the model calculation. Thus, although the evaluated mass spectrum is very well reproduced by the calculation, relatively small deviations lead to a large Chi-squared value.

In summary, from the 59 evaluated mass distributions, we found one case where a shortcoming of the model is clearly proven. In 6 cases, it seems that the evaluation suffers from poor data. In addition, the uncertainties of the evaluation have been underestimated, causing large Chi-squared values. A closer look to these cases does not give indications for a shortcoming of the model but rather for somewhat faulty evaluations. In one case, the measured yields (and thus the evaluated data) are so precise that the uncertainties of the model exceed the uncertainties of the evaluation substantially. This leads to large Chi-squared values, although the mass distribution is well reproduced.

A closer view of the mass distributions reveals some additional somewhat minor problems either in the evaluation or in the model. The calculated yields around symmetry often deviate from the empirical data. The 
prediction of the low yields at symmetry is very demanding due to their high sensitivity to excitation energy and the strong variation from system to system. This is particularly critical for fast-neutron-induced fission, where the neutron-energy distribution in the experiment might be rather broad, and the energy distribution of fissioning systems is weighted with the energy-dependent fission probability of the specific system. Moreover, experimental data in the region of very low yields near symmetry are very scarce, and the uncertainties are large. In the right wing of the left peak for the system ${ }^{237} \mathrm{~Np}\left(\mathrm{n}_{\mathrm{th}}, \mathrm{f}\right)$ appears a structure, which is probably caused by a contamination of the target by a heavier nucleus (see section XI A). Note that the position of the heavy fission-product group is roughly independent of the fissioning nucleus, while the position of the light fission-fragment group moves accordingly. There are several systems, where the outer wings of the evaluated mass distribution appear to have a schematic, unusual shape, probably due to the lack of reliable data (in addition to the systems already mentioned above): ${ }^{250} \mathrm{Cf}(\mathrm{sf}),{ }^{232} \mathrm{U}\left(\mathrm{n}_{\mathrm{th}}, \mathrm{f}\right)$, and ${ }^{237} \mathrm{~Np}(\mathrm{n}, \mathrm{f})$ at $E_{n}=14 \mathrm{MeV}$ are the most prominent cases.

b. Charge Polarization and Emission of Prompt Neutrons: There is a rather limited amount of data on the neutron excess of the fission products. Fig. 50 proves that the mean neutron excess and the fluctuations are well reproduced over the large range from ${ }^{233} \mathrm{U}\left(\mathrm{n}_{\mathrm{th}}, \mathrm{f}\right)$ to ${ }^{249} \mathrm{Cf}\left(\mathrm{n}_{\mathrm{th}}, \mathrm{f}\right)$. The reason for the deviations for $A_{\text {post }}>$ 105 from ${ }^{249} \mathrm{Cf}\left(\mathrm{n}_{\mathrm{th}}, \mathrm{f}\right)$ is not clear, because the resolution of the experiment was insufficient to distinguish the energy-loss signals from the different elements. The data show very nicely the influence of an even-odd staggering, predominantly in the $Z$ distribution. The good reproduction of the mass-dependent mean prompt-neutron multiplicity for ${ }^{237} \mathrm{~Np}(\mathrm{n}, \mathrm{f})$ as a representative for a lighter system and ${ }^{252} \mathrm{Cf}(\mathrm{sf})$ as a representative for a heavier system that will be discussed in Sec. IX D shows that the influence of charge polarization and prompt-neutron emission is correctly modeled in the GEF code. In particular, the transport of the additional energy from the $5.55 \mathrm{MeV}$ neutron to the heavy fragment is correctly reproduced 35].

c. Nuclide Distributions: The isobaric $Z$ distributions shown in Figs. 51 to 56 demonstrate a very good agreement of the GEF calculations with the measured data for the system ${ }^{235} \mathrm{U}\left(\mathrm{n}_{\mathrm{th}}, \mathrm{f}\right)$. For almost all mass chains, the error bars of the evaluation and the error bars from the estimated uncertainties of the model calculation overlap. Due to the good agreement of the mean value and the standard deviation of the isobaric $Z$ distributions also for other systems shown in Fig. 50 one expects that the nuclide distributions of other systems are described with a similar quality.

The good reproduction of the mass-yield distribution and of the isotopic yields of ${ }^{250} \mathrm{Cf}$ at an excitation energy of $45 \mathrm{MeV}$ and an rms angular momentum of $20 \hbar$, in particular the very good agreement of the mean $N / Z$ ratio, proves that the GEF code gives a reliable descrip- tion of the fission-fragment distributions up to $45 \mathrm{MeV}$ and probably even higher energies. Crucial ingredients of the GEF model are a realistic description of the fission probability as a function of excitation energy and angular momentum (see Sec. IX A 2) and of the influence of angular momentum on the width of the fission-fragment mass distribution (see Sec. VIB).

We can state that the GEF model provides rather reliable estimations of the nuclide distributions also for fission from higher excitation energies. As long as one can assume that fission proceeds by the formation of a compound nucleus, it is expected that the GEF model can safely be applied up to excitation energies in the order of $100 \mathrm{MeV}$. Pre-equilibrium emission in neutron-induced reactions and multi-chance fission are considered in the GEF model, see Secs. IV and V, respectively. Other processes like quasi-fission [175], fast fission [176] or transient effects [177, 178] require special considerations with adapted models.

d. Energy Dependence: The relative intensities of the fission fragments at symmetry are most sensitive to the excitation energy of the fissioning system. The general increase of the valley-to-peak ratio of the mass distributions is rather well described by the GEF model. This validates the statistical approach assumed for the population of the fission channels, including the parametrization of the level densities. There are mostly minor deviations in the absolute values, but they do not seem to be systematical.

On the basis of this analysis one can expect that the GEF model is suited to give reliable estimations of the complete fission-fragment yields over a large excitationenergy range, although the data are scarce and incomplete.

\section{Conclusion and Outlook}

The overall quality of the GEF code for predicting the fission-product yields was demonstrated on the basis of all mass distributions of the ENDF B VII evaluation and other data, comprising measured fissionproduct mass distributions, mass-dependent promptneutron yields, mass-chain $Z$ yields and isotopic distributions. Severe shortcomings of the model appeared only for the system ${ }^{229} \mathrm{Th}\left(\mathrm{n}_{\mathrm{th}}, \mathrm{f}\right)$, while deficiencies of the evaluation were found for a number of other systems.

From the careful comparison of the measured and the evaluated data and the predictions of the GEF code it becomes evident that the GEF code can be applied to substantially improve the quality of evaluated data. This aspect will be discussed in more detail in Sec. XI. 


\section{Isomeric Yields}

\section{Comparison with Measured Data}

The angular-momentum distribution cannot be directly measured and is often extracted from the isomeric ratio. In order to reduce the bias due to the model used for the extraction of the angular momentum, the only benchmark on the prediction of the angular momentum detailed here are the isomeric ratios.

The Naik compilation [202, 203] was used as a reference for experimental data. These isomeric ratios are usually extracted from $\gamma$-ray spectroscopy coupled with radiochemistry technique. This technique relies on the values of the branching ratio $I_{\gamma}$ which are often known with an uncertainty larger than 5-10\%, which consequently leads to large uncertainties on the isomeric ratio. Moreover, the nuclei studied have a long life time ( $>1$ minute) and are in the heavy peak. Very few measurements were performed on the light peak. As the angular momentum depends on the mass of the fragment, new measurements on the light peak will be welcome.

The isomeric ratio predicted by the GEF model depends on the mass of the fragment, the deformation of the fragment, the $Z$ parity of the fragment, the spin difference between the isomeric state and the ground state of the fragment, the excitation energy, and the spin of the compound nucleus. These dependencies will be studied in this section.

Fig. 67 represents the ratio of the isomeric yield $\left(Y_{m}\right)$ over the sum of the isomeric yield and the ground-state yield $\left(Y_{m}+Y_{g s}\right)$ for several fission products from the ${ }^{239} \mathrm{Pu}\left(\mathrm{n}_{\mathrm{th}}, \mathrm{f}\right)$ reaction. The GEF prediction agrees with the experimental data within the $1 \sigma$ - uncertainty in the majority of cases. The agreement does not depend on the $Z$ parity : the odd- $Z$ isomeric ratios $(\mathrm{Sb}, \mathrm{I}, \mathrm{Cs}, \mathrm{La})$ are predicted with the same quality as the even- $Z$ isomeric ratios $(\mathrm{Te}, \mathrm{Xe})$. The quality does not seem to be influ-

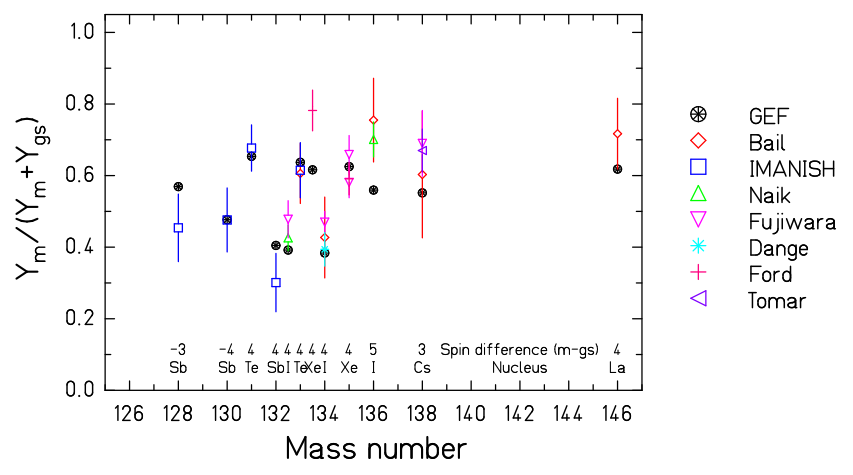

FIG. 67. (Color online) Measured isomeric ratios for several fission products from the ${ }^{239} \mathrm{Pu}\left(\mathrm{n}_{\mathrm{th}}, \mathrm{f}\right)$ reaction $\left.202-204\right]$ are compared with the GEF results. enced by the mass of the fragment, at least on the heavy peak. The difference of the spin of the isomer and the one of the ground state is nearly always the same (values around four in most cases), so the influence of this difference cannot be studied. However, it can be seen on the chain of the Sb isotopes that a negative spin difference is not problematic for the GEF model.

The ratios of the high-spin yield $\left(Y_{h}\right)$ over the sum of the low-spin and the high-spin yields $\left(Y_{l}+Y_{h}\right)$ are compared in Figs. 68 and 69 for fragments produced in the fission of 6 different systems: ${ }^{237} \mathrm{~Np}(5 / 2+)(\mathrm{n}, \mathrm{f}),{ }^{241} \mathrm{Am}$
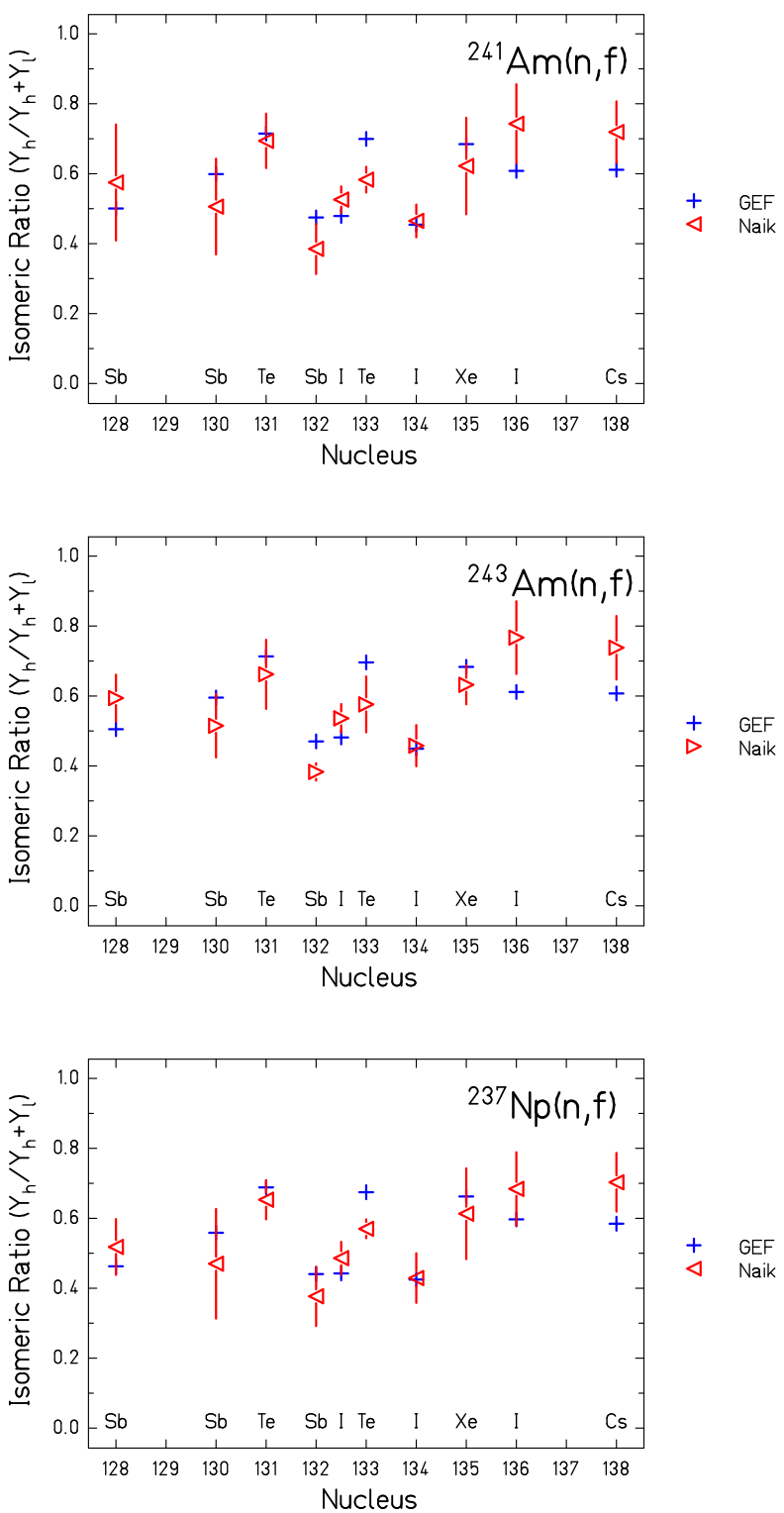

FIG. 68. (Color online) Measured isomeric ratios for several fission products from odd- $Z$ compound nuclei: ${ }^{237} \mathrm{~Np}(5 / 2+)$ $(\mathrm{n}, \mathrm{f}),{ }^{241} \mathrm{Am}(5 / 2-)(\mathrm{n}, \mathrm{f}),{ }^{243} \mathrm{Am}(5 / 2+)(\mathrm{n}, \mathrm{f})$ 205] are compared with the GEF results. 
$(5 / 2-)(\mathrm{n}, \mathrm{f}),{ }^{243} \mathrm{Am}(5 / 2+)(\mathrm{n}, \mathrm{f})$, and ${ }^{232} \mathrm{Th}(0+)(\mathrm{n}, \mathrm{f})$, in fast-neutron-induced fission, ${ }^{235} \mathrm{U}(7 / 2-)\left(\mathrm{n}_{\mathrm{th}}, \mathrm{f}\right)$, and ${ }^{252} \mathrm{Cf}(0+)(\mathrm{sf})$. The conclusions are the same as the previous ones on ${ }^{239} \mathrm{Pu}\left(\mathrm{n}_{\mathrm{th}}, \mathrm{f}\right)$. The agreement between the experimental data and the GEF prediction is good whatever the parity and the spin of the compound nucleus.

In order to extend our benchmark of the GEF prediction as a function of the compound nucleus, four isomeric ratios (the $\mathrm{Sb}$ chain and ${ }^{135} \mathrm{Xe}$ ) are compared for 15 fissioning systems in Figs. 70 and 71 . The ${ }^{132} \mathrm{Sb}$ isomeric ratios are over-predicted whereas the ${ }^{128} \mathrm{Sb}$ ratios are under-predicted. The ${ }^{135} \mathrm{Xe}$ isomeric ratio is well re-
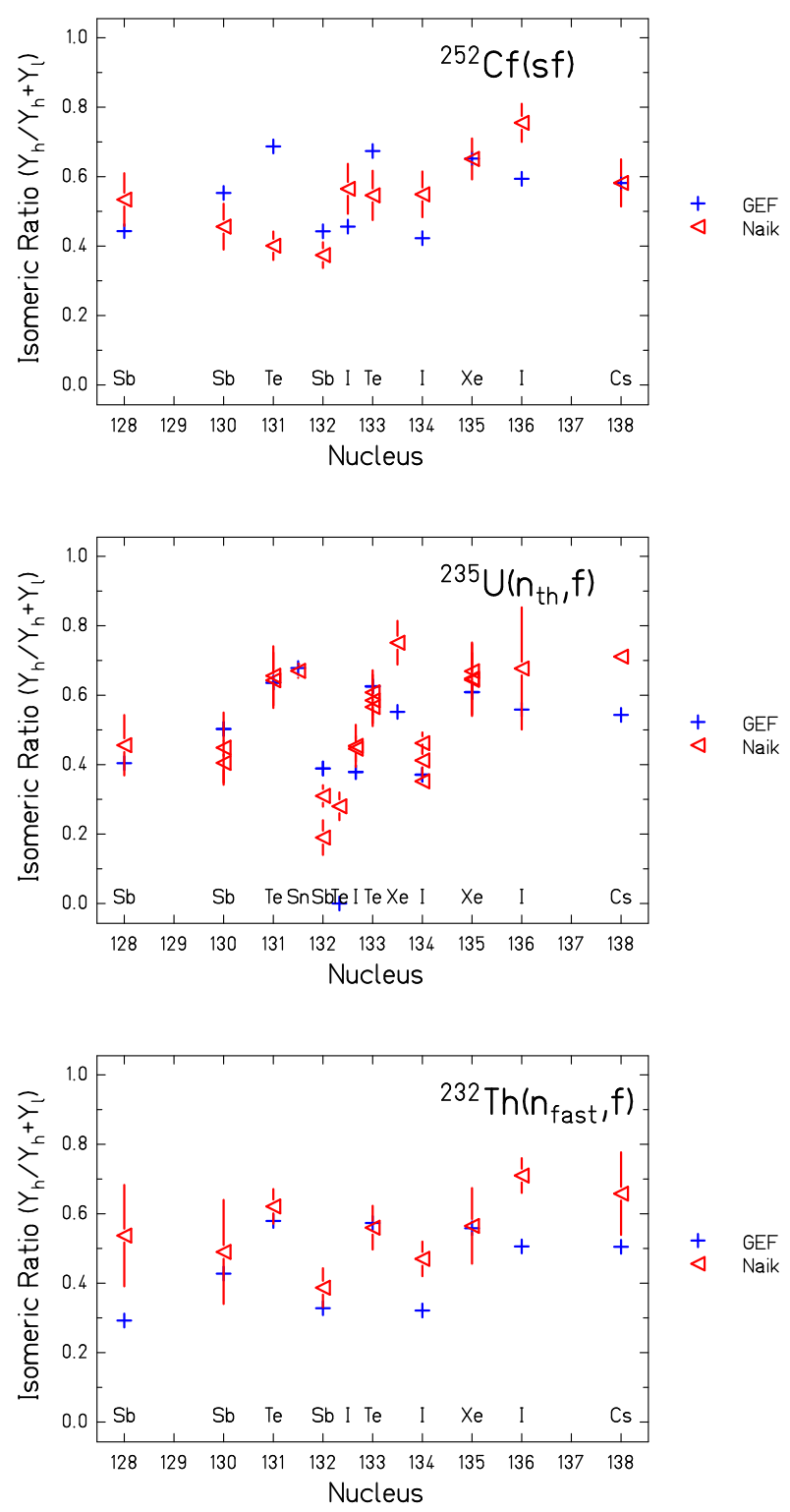

FIG. 69. (Color online) Measured isomeric ratios for several fission fragments from even- $Z$ compound nuclei: ${ }^{232} \mathrm{Th}(0+)$ $(\mathrm{n}, \mathrm{f}),{ }^{235} \mathrm{U}(7 / 2-)\left(\mathrm{n}_{\mathrm{th}}, \mathrm{f}\right),{ }^{252} \mathrm{Cf}(0+)(\mathrm{sf})$ 202] are compared with the GEF results.

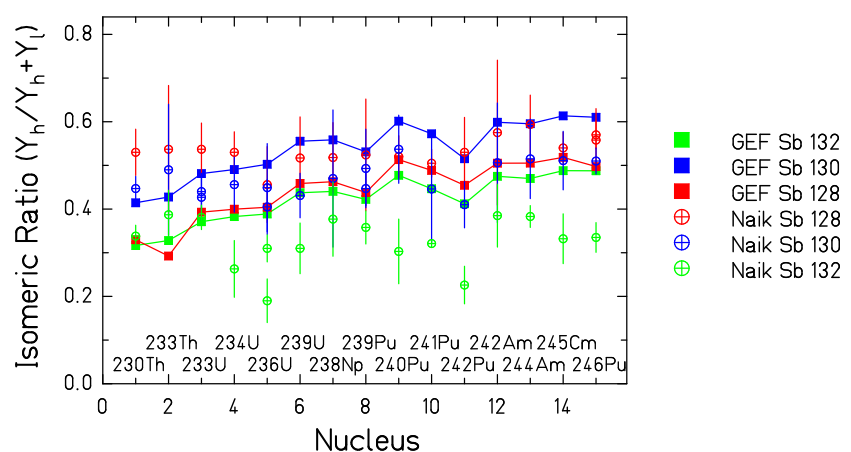

FIG. 70. (Color online) Measured isomeric ratios for the $\mathrm{Sb}$ chain for different fissioning nuclei [202, 203] are compared with the GEF results.

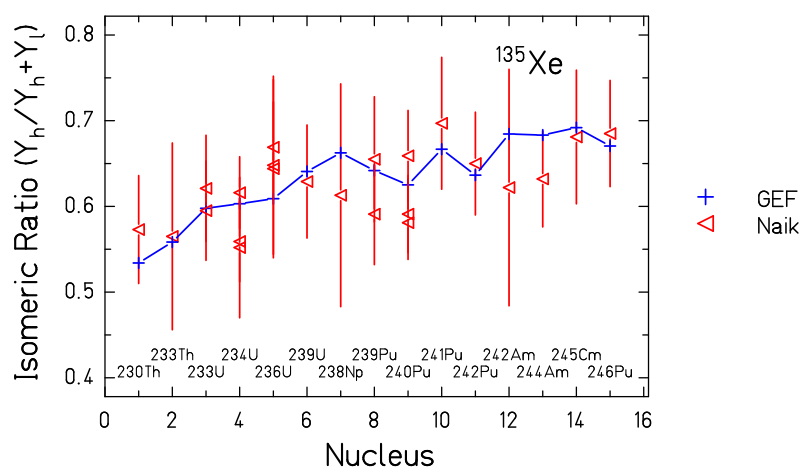

FIG. 71. (Color online) Measured isomeric ratio for ${ }^{135} \mathrm{Xe}$ for different fissioning nuclei [202, 203] is compared with the GEF result.

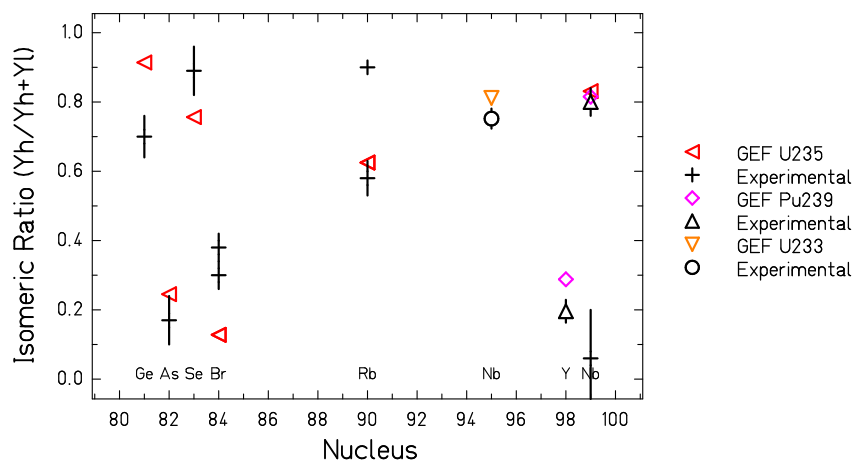

FIG. 72. (Color online) Measured isomeric ratios for light fragments from different thermal-neutron-induced reactions 204, 207, 208] are compared with the GEF results.

produced. In each case, the tendency with the variation of the compound nucleus is good.

Even if few data are available for the light group, Fig. 72 shows that the GEF code tends to overestimate the few ones available on the average. According to 

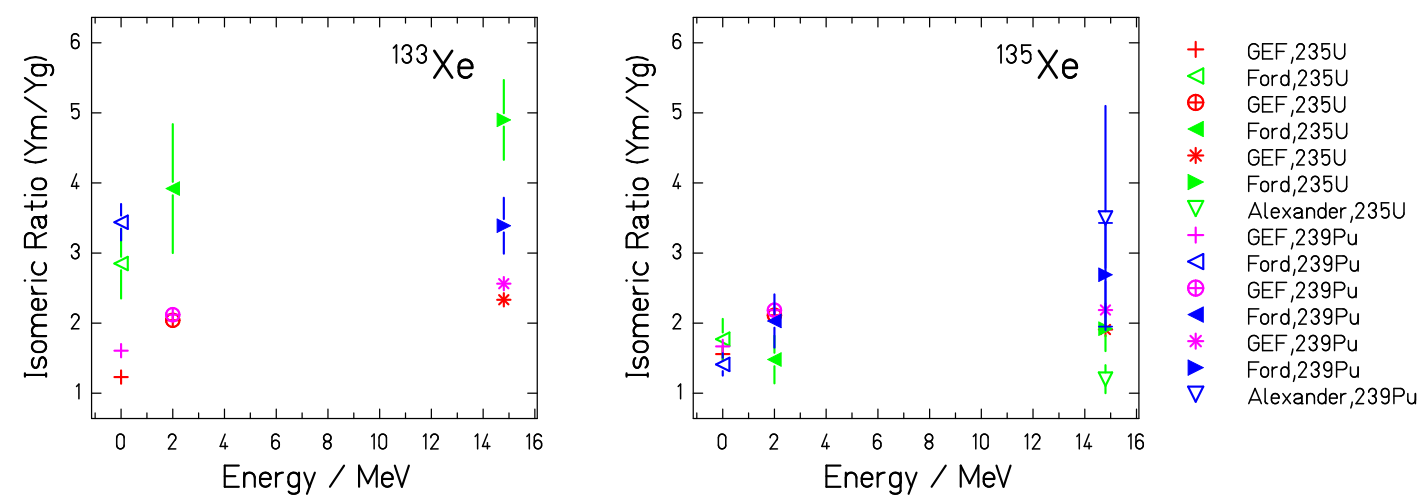

FIG. 73. (Color online) Measured isomeric ratios for ${ }^{133} \mathrm{Xe}$ and ${ }^{135} \mathrm{Xe}$ from ${ }^{235} \mathrm{U}(\mathrm{n}, \mathrm{f})$ and ${ }^{239} \mathrm{Pu}(\mathrm{n}, \mathrm{f})$ for different neutron energies [209] are compared with the GEF results.

the experimental data on the heavy peak that indicate a small influence of the compound nucleus on the isomeric ratio, the experimental ${ }^{99} \mathrm{Nb}$ value for ${ }^{235} \mathrm{U}$ can be wrong as the experimental ${ }^{99} \mathrm{Nb}$ value for ${ }^{239} \mathrm{Pu}$ is the complementary to 1 . This can be due to the inversion $Y_{m} / Y_{g s}=Y_{l} / Y_{h}$ for the nucleus contrary to a lot of nuclei where $Y_{m} / Y_{g s}=Y_{h} / Y_{l}$.

In order to study the influence of the excitation of the compound nucleus on the isomeric ratio, the ${ }^{135} \mathrm{Xe}$ and ${ }^{133} \mathrm{Xe}$ isomeric ratios are also compared as a function of the excitation energy with the Ford measurement (with thermal, 2-MeV, and 14-MeV neutrons) in Fig. 73 Ford observed an increase of the $Y_{m} / Y_{g s}$ ratio for ${ }^{133} \mathrm{Xe}$ whereas he saw no increase for ${ }^{135} \mathrm{Xe}$. The GEF code does not reproduce the nearly constant behavior before $3 \mathrm{MeV}$. A larger number of data is however required to extend this observation more especially in the range $E_{n}=2-14$ $\mathrm{MeV}$.

Photo-fission reactions also give some hints that the excitation energy does not have a huge influence on the isomeric ratio at least in the range $E^{*}=9.7-14.1 \mathrm{MeV}$ $\left(E_{n}=4-8 \mathrm{MeV}\right)$. Fig. 74 shows the isomeric ratio for ${ }^{134} \mathrm{I}$ for ${ }^{235} \mathrm{U}(\gamma, \mathrm{f})$ and ${ }^{238} \mathrm{U}(\gamma, \mathrm{f})$ as a function of the excitation energy of the compound nucleus. The experimental ratios are nearly constant for both compound nuclei in the domain $E^{*}=9.7-14.1 \mathrm{MeV}$. The GEF predictions are also nearly constant. The excitation-energy dependence of the isomeric ratio as parametrized in the GEF code seems to be correct, although the absolute values are underestimated by up to $20 \%$ in some cases.

In conclusion, the GEF prediction, by essentially assuming that the angular momentum of the fragments is created by the statistical population of single-particle and collective states according to the fragment temperature at scission, is in general in very good agreement with the data, both in the absolute values and in the systematic variations for various systems and as a function of energy.

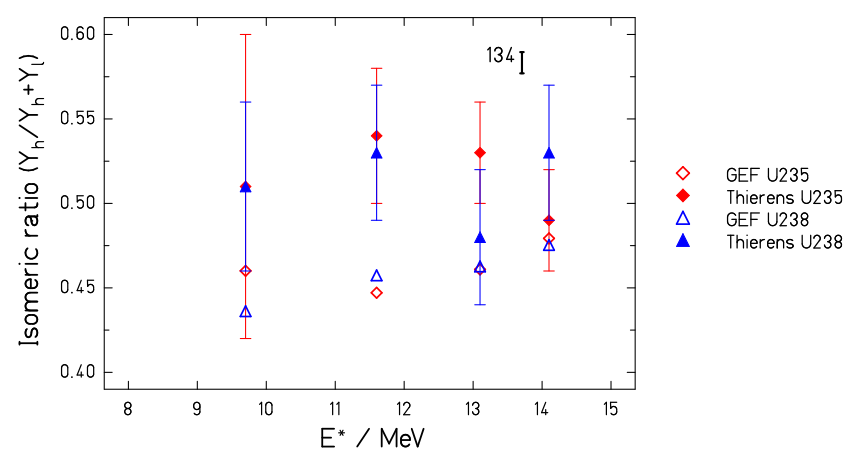

FIG. 74. (Color online) Isomeric ratio for ${ }^{134}$ I from photofission. Measured isomeric ratios for ${ }^{134} \mathrm{I}$ from ${ }^{235} \mathrm{U}(\gamma, \mathrm{f})$ and ${ }^{238} \mathrm{U}(\gamma, \mathrm{f})$ at different excitation energies [210] are compared with the GEF results.

\section{Even-odd Staggering in Angular Momentum}

In several experiments involving spontaneous, as well as thermal to fast neutron-induced fission, Naik and collaborators, see e.g. Refs. 202, 203, 205, 206 and therein, concluded that two features have significant impact on the understanding of angular-momentum generation in fission. They found that the angular momenta $J$ of fragments with spherical $N=82$ shell and those with even $Z$ are systematically lower compared to the angular momenta of fragments with $N$ out-of-shell and odd $Z$ number. The very strong even-odd staggering in $J$ as a function of $Z$ found by Naik and collaborators is very intriguing and most appealing. At first glance, an even-odd effect in fission-fragment angular momenta sounds surprising. Indeed, at scission the fragments are excited, and it is only after cooling down to the yrast line, that structural and/or even-odd effects are expected to play a role. By that time, memory of the initial stage is thus in 
principle lost. The conclusion by Naik and collaborators is based on the measurements of independent isomeric yield ratios (IYR) and subsequent analysis with a statistical model. The idea is that the probability for populating an isomer of a given spin depends on the initial angular-momentum distribution of the fragment. Calculations based on the statistical model are performed with a trial $J$ distribution, by adjusting the rms $J$ value, until the experimental IYRs were reproduced. By this method, Naik et al. found a very pronounced even-odd staggering as a function of $Z$, with $J_{r m s}$ being up to 4 $\hbar$ units larger for odd- $Z$ fragments than for even- $Z$ s. As observed in Sec. IX C 1, a consistent description of the experimental IYRs is achieved with the GEF code. To do so, it was found that the required magnitude of the aforementioned even-odd staggering is rather weak (about 0.4 $\hbar$ units), in disagreement with the strong effect deduced by Naik et al. (see also Sec. III A). In addition, a survey of the experimental data shows that the IYRs do not display any noticeable systematic staggering as function of $Z$. According to i) the physics arguments described above, ii) the absence of noticeable even-odd effects in the IYRs themselves, and iii) the weak staggering needed in GEF to describe consistently the experimental IYRs, we suggest that the large effect inferred by Naik et al. is strongly model-dependent. A few previous experiments 136 based on the same experimental IYR technique and on a similar statistical-model analysis yielded also an even-odd effect in $J$. Yet, the staggering was weaker than obtained in Refs. [202, 203, 205, 206]. The difference is understood here as due to differences in detail of the statistical analysis, and further corroborates the model-dependence of the extracted $J$ and its dependence on $Z$. We finally note that Monte-Carlo Hause-Feshbach (MCHF) calculations of the Los Alamos group [142] predict $J$ values similar to those derived from GEF and which do not exhibit any systematic even-odd staggering with $Z$. Furthermore, FIFRELIN calculations [211] assuming a simple flat distribution for the $J$ distribution of the fragments at scission yield a distribution after neutron and statistical gamma emission that is still exempt of a staggering as function of $Z$. Thus, the discrepancy between the interpretation in Refs. 202, 203, 205, 206] and the GEF results cannot be attributed to the neglecting of the angular momentum carried away along the fragment de-excitation after scission in the GEF model. Rather, we suggest the strong model-dependence of the staggering extracted by Naik et al..

\section{Prompt-neutron Multiplicities}

The multiplicities of prompt fission neutrons contain valuable information and, thus, provide a stringent test for the understanding of the fission process. Moreover, this quantity is very important for nuclear technology. The prompt-neutron multiplicity is rather directly connected with the excitation energies of the fragments. For- tunately, prompt-neutron yields have been measured for many fissioning systems. In a few cases, the variation of the prompt-neutron yield as a function of excitation energy and fragment mass has been determined.

\section{System Dependence}

There exist extended systematics of prompt-neutron multiplicities for spontaneous fission and for thermalneutron-induced fission. They are compared in Figs. 75 and 76 with results of the GEF code. It is obvious that the data cannot be parametrized by a simple function of a macroscopic parameter, e.g. the fissility parameter $Z^{2} / A$ or the Coulomb parameter $Z^{2} / A^{1 / 3}$.

a. Spontaneous Fission: In spontaneous fission, the most striking structural effects are (i) the approximate constancy of the neutron multiplicity for the different $\mathrm{Pu}$ isotopes that deviates from the average slope of the other isotopic chains and (ii) the decrease towards the heaviest Fm isotopes. The first effect is the consequence of the large yield of the standard 1 fission channel, which is characterized by a $17 \mathrm{MeV}$ higher TKE value [181] and a correspondingly reduced TXE. Let us recall that the large yield of the $\mathrm{S} 1$ fission channel for these nuclei is attributed to the influence of a shell in the light fragment around $Z=42$ in the GEF model. The yield of the standard 1 channel increases gradually from ${ }^{236} \mathrm{Pu}$ to ${ }^{244} \mathrm{Pu}$, which explains the almost constant prompt-neutron multiplicity for the $\mathrm{Pu}$ isotopes. The reduction of the prompt-neutron yield due to the increasing yield of the S1 channel compensates the general trend that shows an increase of the prompt-neutron yield with increasing mass number, as can be seen in the behavior of the uranium, curium, and californium isotopic sequences. The second effect reflects the rather sudden appearance above ${ }^{256} \mathrm{Fm}$ of a narrow symmetric fission component with TKE values which are higher by about $30 \mathrm{MeV}$ [212].

The measured values are very well reproduced by the GEF model with a few exceptions. The experimental value for ${ }^{232} \mathrm{Th}$ has a large uncertainty, and the one for ${ }^{253}$ Es was reported without mentioning the uncertainty range. Thus, these values may be considered with some caution. Moreover, the increase of the measured values from ${ }^{256} \mathrm{Fm}$ to ${ }^{257} \mathrm{Fm}$ seems to be in conflict with the increase of the measured yield of the narrow symmetric component and its high total kinetic energy, because the TKE and the TXE are connected through the $\mathrm{Q}$ value by energy conservation. The expected further decrease of the prompt-neutron yield towards ${ }^{258} \mathrm{Fm}$ is demonstrated by the calculated value in Fig. 75. Therefore, the measured value for ${ }^{256} \mathrm{Fm}$ may be doubted. The rms deviation between the remaining 19 experimental values and the corresponding calculations amounts to 0.086. This is also the order of magnitude of the expected uncertainty for the predictions of the prompt-neutron yields of nuclei in the vicinity of the systems shown in Fig. 75. Thus, the GEF model is expected to be able to estimate the 
Prompt-neutron yields for spontaneous fission

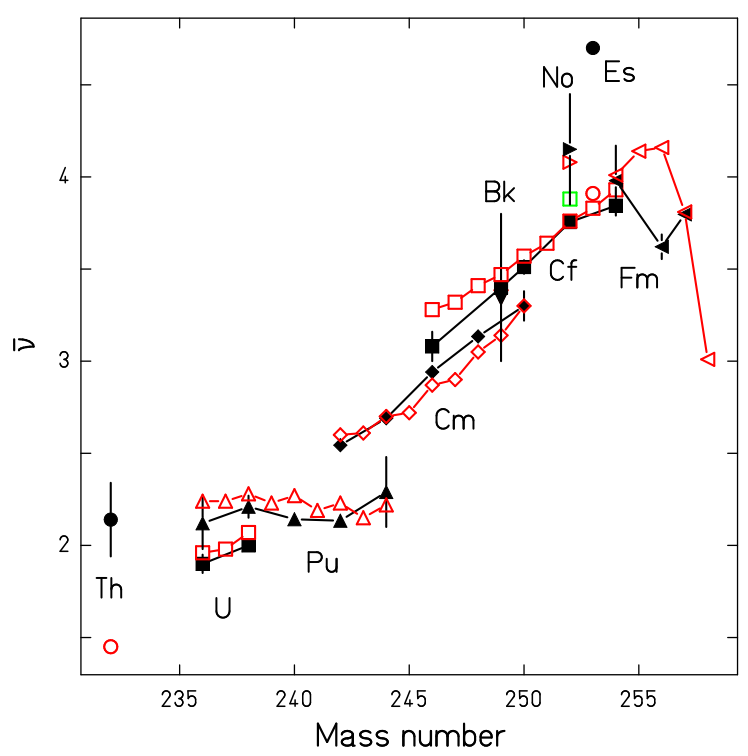

FIG. 75. (Color online) Systematics of prompt-neutron multiplicities for spontaneous fission. Measured mean promptneutron multiplicities for spontaneous fission (black full symbols) as a function of the mass number of the fissioning nucleus [213] in comparison with the result of the GEF model (red open symbols). Experimental error bars are not shown when they are smaller than the symbols. The value for ${ }^{253} \mathrm{Es}$ is reported without an experimental uncertainty.

prompt-neutron multiplicity for spontaneous fission with a precision better than 0.1 units.

b. Thermal-neutron-induced Fission: In the case of thermal-neutron-induced fission, the situation is more complex. A number of data are rather well reproduced by the GEF model, see Fig. 76, but there are also large deviations. The value for ${ }^{232} \mathrm{U}$ reported in Ref. 213 deviates by exactly one neutron from the value obtained by the GEF model. Unfortunately, Ref. 213] cites another publication [215] that is not easily accessible. Therefore, the possibility of a misprint, which is tentatively assumed in Fig. 76, could not be verified. For the large discrepancies for ${ }^{229} \mathrm{Th},{ }^{233} \mathrm{U},{ }^{238} \mathrm{Pu},{ }^{241,243} \mathrm{Am}$, and ${ }^{245,247} \mathrm{Cm}$ there is no obvious explanation. There is no obvious systematics in these deviations. It is striking that the data for the following systems with easily available target material $\left({ }^{235,238} \mathrm{U},{ }^{237} \mathrm{~Np},{ }^{239,241} \mathrm{Pu},{ }^{252} \mathrm{Cf}\right)$, and also ${ }^{232} \mathrm{U}$ are very well reproduced.

The situation is not clear. More experimental work would be desirable in order to better understand the structural effects, which are eventually responsible for the observed deviations, and in order to verify the result and to exclude possible systematic uncertainties of one or the other experiment. The rms deviation between all 21 experimental values, including those with large error bars, amounts to 0.17 , which is about twice the value found for

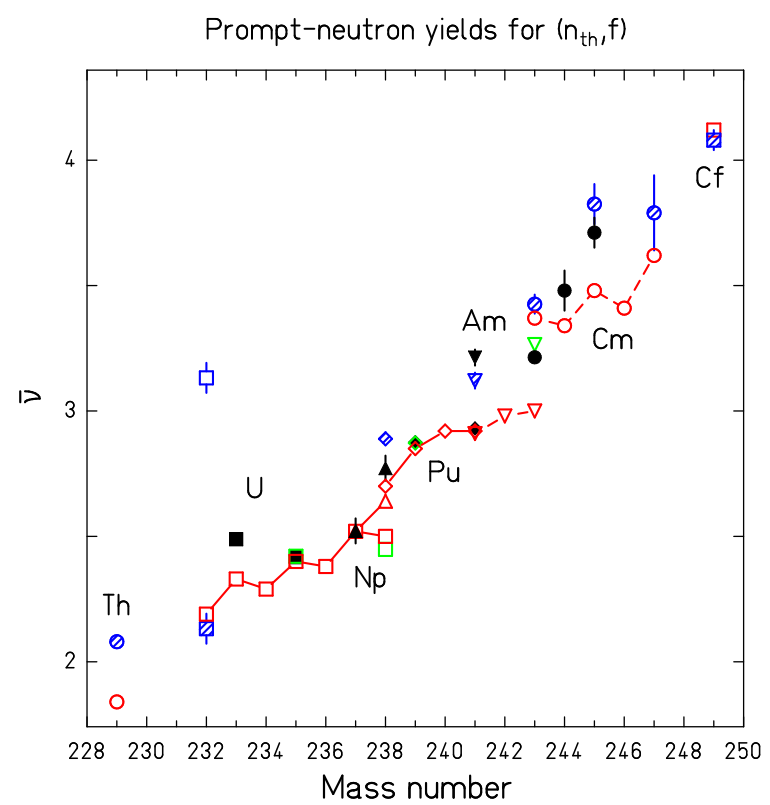

FIG. 76. (Color online) Systematics of prompt-neutron multiplicities for $\mathrm{n}_{\mathrm{th}}$-induced fission. Measured mean promptneutron multiplicities for thermal-neutron-induced fission as a function of the mass number of the target nucleus 214] (black full symbols), 213] (blue shaded symbols), and [179] (green open symbols) in comparison with the result of the GEF model (red open symbols). We assumed that the value 3.132 for ${ }^{232} \mathrm{U}$ given in [213] (blue open symbol) is wrong due to a misprint. The tentatively corrected value (2.132) is marked by a blue shaded symbol. Experimental error bars are not shown when they are smaller than the symbols.

spontaneous fission. For ${ }^{232} \mathrm{U}$, the tentatively corrected value was used. Thus, the GEF model is expected to be able to estimate the prompt-neutron multiplicity for thermal-neutron-induced fission with a precision better than 0.2 units.

\section{Energy Dependence}

For a few target nuclei, the prompt-neutron multiplicity has been measured in neutron-induced fission as a function of the incident-neutron energy. Great part of these data are compared with the results of the GEF model in Fig. 77. The experimental data are taken from Ref. 213]. Only part of the data are shown if they overlap in order not to overload the figure. The overall slope of the neutron multiplicity as a function of neutron energy is well reproduced by the model. The data for the two systems ${ }^{235} \mathrm{U}(\mathrm{n}, \mathrm{f})$ and ${ }^{239} \mathrm{Pu}(\mathrm{n}, \mathrm{f})$, which have been studied most extensively, are very well reproduced over the whole energy range up to almost $30 \mathrm{MeV}$. The data for ${ }^{232} \mathrm{Th}(\mathrm{n}, \mathrm{f})$ show a structure at the onset of secondchance fission, which is well reproduced by the model as well. The strong increase of the neutron multiplicity 
just above the threshold for second-chance fission can be explained by the fact that second-chance fission is only possible in this energy range, if the kinetic energy of the emitted pre-fission neutron is so low that the excitation energy of the daughter nucleus falls above its fission barrier. Thus, the average prompt-neutron energy is exceptionally low, and the corresponding neutron multiplicity is exceptionally high. Also, another peculiarity of this system, the weak increase of the prompt-neutron multiplicity in the low-energy range up to $3 \mathrm{MeV}$ is present in the model results. This effect is connected with the fact that for this even-even nucleus low incident neutron energies lead to excitation energies around the fission barrier. In the tunneling regime, at energies below the fission barrier, the TXE values do not directly follow the variations of the initial excitation energy. A similar, however much weaker structure than in ${ }^{232} \mathrm{Th}(\mathrm{n}, \mathrm{f})$ at the onset of firstchance fission of ${ }^{238} \mathrm{U}(\mathrm{n}, \mathrm{f})$ in the calculated values is not seen in the data. The structures seen in the model results at the threshold for third-chance fission near $15 \mathrm{MeV}$ for ${ }^{235} \mathrm{U}(\mathrm{n}, \mathrm{f})$ and ${ }^{239} \mathrm{Pu}(\mathrm{n}, \mathrm{f})$ cannot be compared, because there are no data measured between 15 and $22 \mathrm{MeV}$.

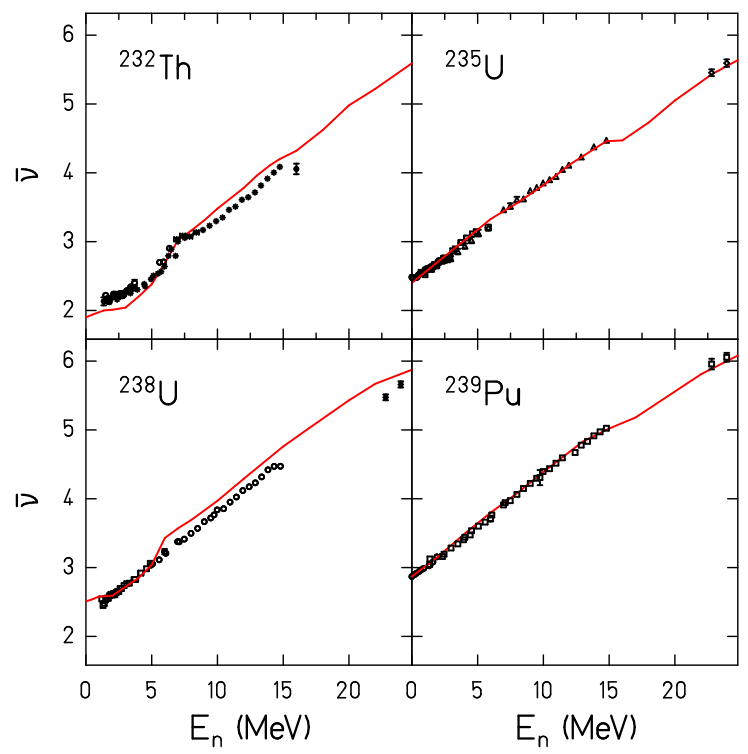

FIG. 77. (Color online) Energy dependence of mean promptneutron multiplicities. Measured prompt-neutron multiplicity for ${ }^{232} \mathrm{Th}(\mathrm{n}, \mathrm{f}),{ }^{235} \mathrm{U}(\mathrm{n}, \mathrm{f}),{ }^{238} \mathrm{U}(\mathrm{n}, \mathrm{f})$, and ${ }^{239} \mathrm{Pu}(\mathrm{n}, \mathrm{f})$ (black symbols, different symbols are used for different experiments) as a function of neutron energy (data from Ref. [213]) in comparison with the result of the GEF model (red line).

It is interesting to note that the energy-dependent prompt-neutron multiplicity is perfectly reproduced by the GEF model for the odd- $A$ targets ${ }^{235} \mathrm{U}(\mathrm{n}, \mathrm{f})$ and ${ }^{239} \mathrm{Pu}(\mathrm{n}, \mathrm{f})$, in contrast to the even- $A$ targets ${ }^{232} \mathrm{Th}(\mathrm{n}, \mathrm{f})$ and ${ }^{238} \mathrm{U}(\mathrm{n}, \mathrm{f})$, where the neutron yield is overestimated above the threshold for second-chance fission. This prob- lem is probably connected with the difficulties in describing the fission probabilities of systems with relatively low fissility and high neutron-separation energies, which were already reported in Sec. IX A. In general, the specific behavior of the prompt-neutron multiplicity at the onset of a higher-chance fission strongly depends on the behavior of the fission probability around the fission threshold, which shows a gradual increase in part of the systems and a more or less pronounced peak structure in other systems. In the first case, the mean neutron energy tends to increase, in the second case it tends to decrease with the opening of another fission chance. This feature strongly depends on structural effects in the level density (see the discussion in Sec. IX A). In addition, the neutron yield for ${ }^{232} \mathrm{Th}(\mathrm{n}, \mathrm{f})$ is underestimated at incident-neutron energies below $5 \mathrm{MeV}$. Possibly, this problem is related in some way with the discrepancies observed in the fragment yields from the fission of several thorium isotopes.

\section{Fragment-mass Dependence}

In the actinides, the prompt-neutron multiplicity has the typical saw-tooth behavior as a function of fragment mass. Fig. 78 shows the measured data for the system ${ }^{237} \mathrm{~Np}(\mathrm{n}, \mathrm{f})$ for two incident-neutron energies. The data for ${ }^{252} \mathrm{Cf}(\mathrm{sf})$ are shown in Fig. 79. The data are rather well reproduced by the GEF model.

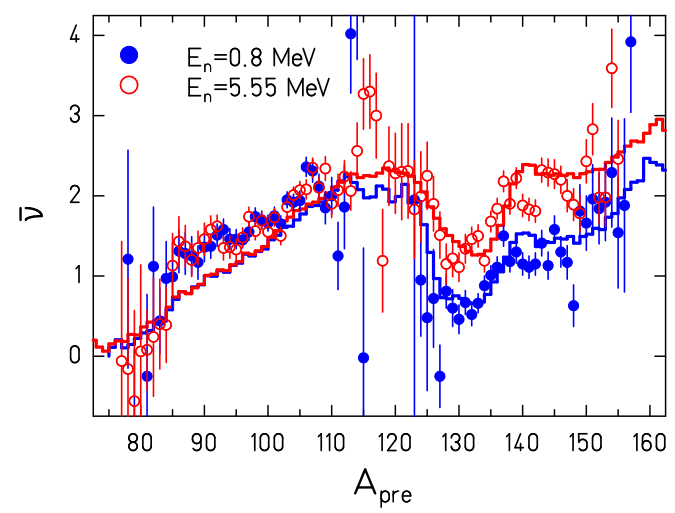

FIG. 78. (Color online) Measured prompt-neutron yield in ${ }^{237} \mathrm{~Np}(\mathrm{n}, \mathrm{f})$ as a function of pre-neutron mass at two different incident-neutron energies [80] (data points) in comparison with the result of the GEF model (histograms).

There are two prominent features in the model: First, the increasing yields are caused by the fragment deformation at scission which increases with the fragment mass in the range of the light and in the range of the heavy fragments. This feature is a consequence of a general characteristics of shells in deformed nuclei, already mentioned in Sec. VID: These shells extend over a broad range of neutron, respectively proton number, but the optimum deformation is correlated with the size of the 


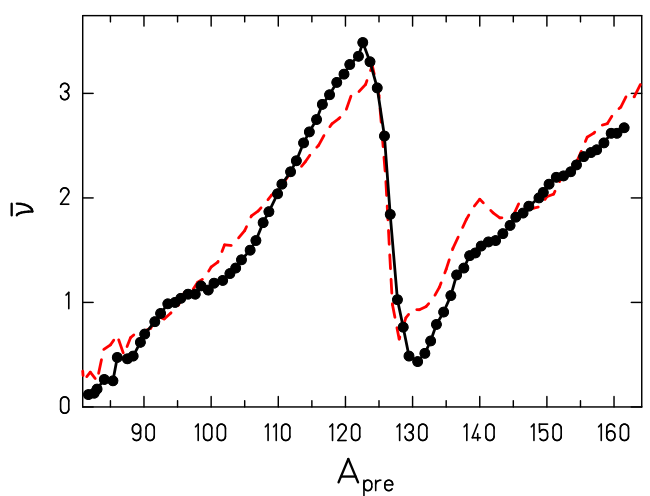

FIG. 79. (Color online) Measured prompt-neutron yield in ${ }^{252} \mathrm{Cf}(\mathrm{sf})[216]$ as a function of pre-neutron mass (data points) in comparison with the result of the GEF code (dashed line). The experimental uncertainties are smaller than the symbols.

system [74, 76]. Secondly, the intrinsic excitation energy at scission is subject to energy sorting [35]. Thus, the higher incident neutron energy in ${ }^{237} \mathrm{~Np}(\mathrm{n}, \mathrm{f})$ leads to an increased neutron multiplicity in the heavy fragment, only.

It is remarkable that the data of the two systems are well reproduced by the model with the fundamental assumption that the fragment deformation at scission is a unique function of the fragment shells.

\section{Multiplicity Distributions}

The distribution of prompt-neutron multiplicities provides a test for the fluctuation of the total excitation energy of the fragments. In the GEF model, the largest contribution to these fluctuations is caused by the distribution of fragment deformations around the equilibrium value at scission. The distributions for ${ }^{239} \mathrm{Pu}\left(\mathrm{n}_{\mathrm{th}}, \mathrm{f}\right)$ and ${ }^{252} \mathrm{Cf}(\mathrm{sf})$ shown in Fig. 80 are perfectly reproduced, whereas the calculated distribution for ${ }^{235} \mathrm{U}\left(\mathrm{n}_{\mathrm{th}}, \mathrm{f}\right)$ is slightly too narrow.

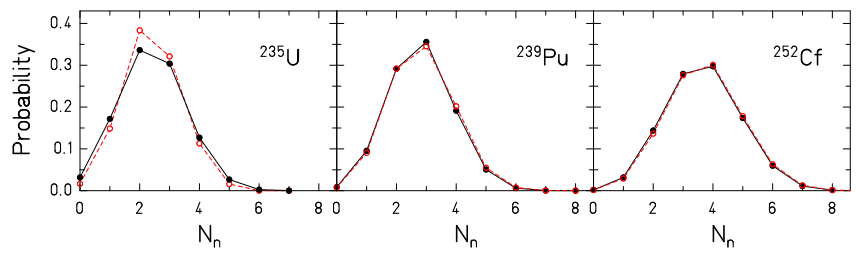

FIG. 80. (Color online) Measured distribution of promptneutron multiplicities in ${ }^{235} \mathrm{U}\left(\mathrm{n}_{\mathrm{th}}, \mathrm{f}\right),{ }^{239} \mathrm{Pu}\left(\mathrm{n}_{\mathrm{th}}, \mathrm{f}\right)$ and ${ }^{252} \mathrm{Cf}(\mathrm{sf})$ [217, 218] (black full points) in comparison with the result of the GEF model (red open points).

\section{Conclusion}

The manifold data on prompt-neutron multiplicities show a large variety of gross and subtle features. The GEF model is able to reproduce most of them with a satisfactory quality. Even more importantly, the model traces these features back to peculiar aspects of the physics governing the fission process. This way, the model provides a link to other observables which are consistently described by the model.

\section{E. Prompt-neutron Energies}

\section{Key Systems}

a. Prompt-neutron Energy Spectra: The experimental prompt-fission-neutron energy spectra for the systems ${ }^{235} \mathrm{U}\left(\mathrm{n}_{\mathrm{th}}, \mathrm{f}\right)$ [219] and ${ }^{252} \mathrm{Cf}(\mathrm{sf})$ [220] are compared with results of the GEF code in Fig. 81. In order to better visualize the deviations, the lower panels show a reduced presentation with the spectra normalized to a Maxwellian distribution with the parameter $T=1.32 \mathrm{MeV}$.

In this calculation, the de-excitation of the separated fragments has been obtained within the statistical model. It is assumed that both the emission of neutrons and the emission of E1 gammas do not change the angular momentum on the average, which seems to be a good approximation in the relevant angular-momentum range [124]. When the yrast line is reached, the angular momentum is carried away by a cascade of E2 gammas. The inverse neutron absorption cross-section has been described by the parametrization from [140]. Since the fast-neutron energy spectrum in fission is composed of the contributions from many emitting fragments, the use of this global description that is computed very quickly is probably not too critical. Gamma competition at energies above the neutron separation energy was considered. The gamma strength of the giant dipole resonance (GDR) following the description proposed in Ref. [141] was applied. The nuclear-level-density description as defined in Sec. III B is used. It is repeated here for convenience. The nuclear level density was modeled by the constant-temperature description of von Egidy and Bucurescu [64] at low energies. The level density was smoothly joined at higher energies with the modified Fermi-gas description of Ignatyuk et al. [65, 66] for the nuclear-state density

$$
\rho_{F G}=C_{F G} \frac{e^{2 \sqrt{\tilde{a} U}}}{\tilde{a}^{1 / 4} U^{5 / 4}}
$$

with $U=E+E_{\text {cond }}+\delta U(1-\exp (-\gamma E)), \quad \gamma=$ $0.055 / \mathrm{MeV}$ and the asymptotic level-density parameter $\tilde{a} /\left(\mathrm{MeV}^{-1}\right)=0.078 A+0.115 A^{2 / 3}$. The shift parameter $E_{\text {cond }}=2 \mathrm{MeV}-n \Delta, \Delta=12 \mathrm{MeV} / \sqrt{A}$ with $n=0,1,2$, for odd-odd, odd- $A$ and even-even nuclei, respectively, as proposed in Ref. 63]. $\delta U$ is the ground-state shell correction. A constant spin-cutoff parameter was used. The 

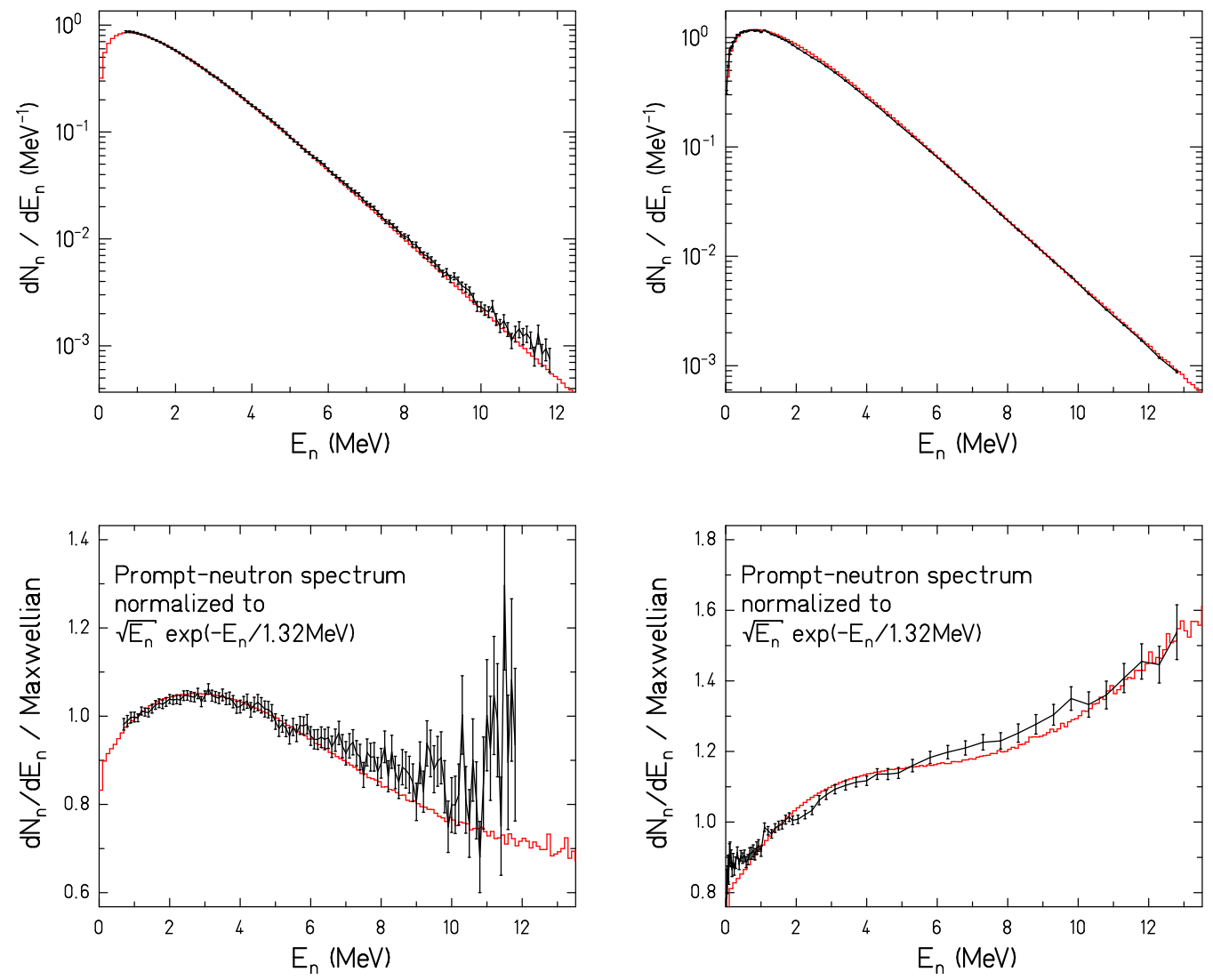

FIG. 81. (Color online) Experimental prompt-fission-neutron energy spectra (black lines and error bars) for ${ }^{235} \mathrm{U}\left(\mathrm{n}_{\mathrm{th}}, \mathrm{f}\right)$ 219] (left panels) and ${ }^{252} \mathrm{Cf}(\mathrm{sf})$ [220] (right panels) in comparison with the result of the GEF model (red histograms) in logarithmic scale. In the lowest panels, all spectra have been normalized to a Maxwellian with $T=1.32 \mathrm{MeV}$.

matching energy is determined from the matching condition (continuous level-density values and derivatives of the constant-temperature and the Fermi-gas part). Values slightly below $10 \mathrm{MeV}$ are obtained. The matching condition also determines the scaling factor $C_{F G}$ for the Fermi-gas part. It is related to the collective enhancement of the level density.

The resulting prompt-neutron energy spectra are shown in Fig. 81. The transformation of the neutronenergies into the laboratory frame was performed considering the acceleration phase [221, 222] after scission by a numerical trajectory calculation. The mean prescission total kinetic energy was assumed to be $40 \%$ of the potential-energy gain from saddle to scission derived by Asghar and Hasse [94] as

$$
<T K E>_{\text {pre }}=0.032\left(Z^{2} / A^{1 / 3}-1527\right) \mathrm{MeV}
$$

with a standard deviation of the same amount. The distribution was truncated at negative values.

The good reproduction of the measured neutron energy spectra, especially for the lighter system ${ }^{235} \mathrm{U}\left(\mathrm{n}_{\mathrm{th}}, \mathrm{f}\right)$, does not give indication for additional neutron emission at scission $223-226]$.

The emission during the acceleration phase is stronger for the system ${ }^{252} \mathrm{Cf}(\mathrm{sf})$, since higher excitation energies and, thus, shorter emission times are involved in this system. Neutron emission during fragment acceleration reduces especially the laboratory energies of the first neutrons emitted at short times from the most highly excited fragments in ${ }^{252} \mathrm{Cf}(\mathrm{sf})$ and allows for a decently consistent description of the two systems with the GEF code, using the same parameter set. Experimental prompt-fissionneutron energy spectra of the systems ${ }^{239} \mathrm{Pu}\left(\mathrm{n}_{\mathrm{th}}, \mathrm{f}\right)$ and ${ }^{240} \mathrm{Pu}(\mathrm{sf})$ are compared with the result of the GEF code in Figs. 82 and 83, again using the same model parameters. Obviously, the data are very well reproduced.

Finally, the prompt-neutron energy spectra for two systems, ${ }^{235} \mathrm{U}\left(\mathrm{n}_{\text {th }} \mathrm{f}\right)$ and ${ }^{239} \mathrm{Pu}\left(\mathrm{n}_{\mathrm{th}}, \mathrm{f}\right)$, are compared with the ENDF/B-VII.1 evaluation in Fig. 84. The calculated spectrum is shown with a variable bin size, see Table XIII as an objective measure to smooth the statistical fluctuations. The logarithmic energy scale shows clearly the general behavior in the low-energy region and in the high-energy tail. One can observe sizable differences for 


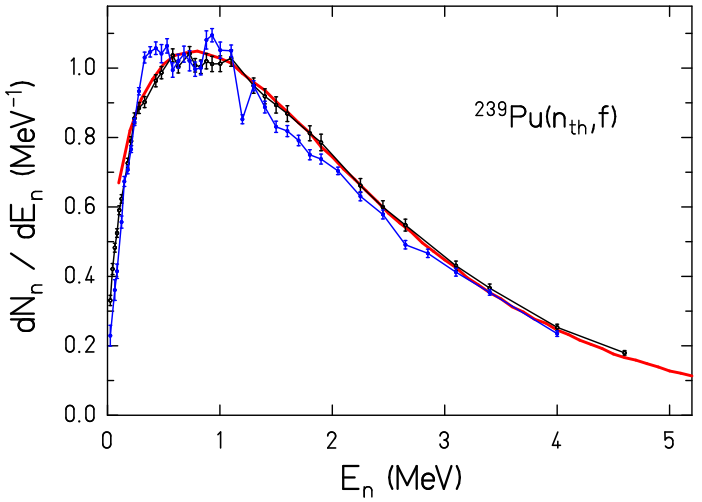

FIG. 82. (Color online) Experimental prompt-fission-neutron energy spectrum for the system ${ }^{239} \mathrm{Pu}\left(\mathrm{n}_{\mathrm{th}}, \mathrm{f}\right)$ from ref. 227] (black open symbols) and from 228] (blue full symbols) in comparison with the result of the GEF code (red thick full line). The calculated spectrum was normalized to the measured total neutron multiplicity $(\bar{\nu}=2.88[217])$. The measured spectra are slightly scaled for minimizing the overall deviations from the calculated spectrum in order to better compare the spectral shapes.

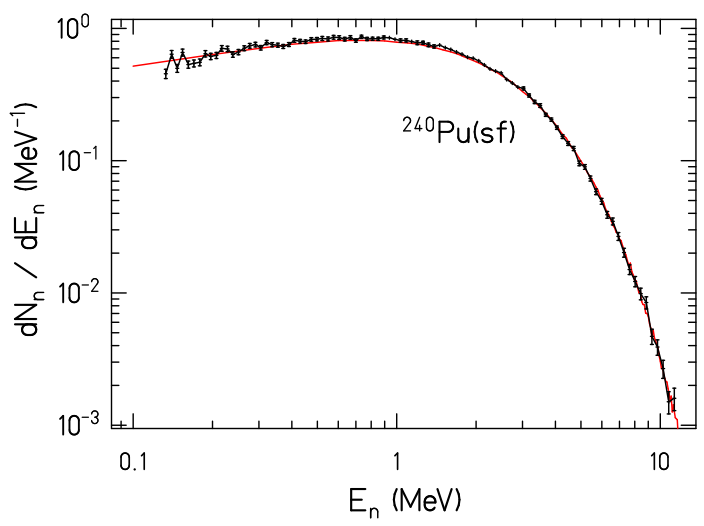

FIG. 83. (Color online) Experimental prompt-fission-neutron energy spectrum for the system ${ }^{240} \mathrm{Pu}(\mathrm{sf})$ from Ref. 229] (black symbols) in comparison with the result of the GEF code (red line). The measured data were scaled to the height of the calculated spectrum. Since the experiment covers especially well the lower-energy range, a double-logarithmic presentation was chosen.

${ }^{235} \mathrm{U}\left(\mathrm{n}_{\mathrm{th}} \mathrm{f}\right)$, in particular in the low-energy region. The fluctuations of the calculated spectrum in the order of $2 \%$ below $0.1 \mathrm{MeV}$ and above $10 \mathrm{MeV}$ can be explained by statistical fluctuations of the Monte-Carlo calculations, considering that the number of counts per bin is about 3000. However, the shoulder around $15 \mathrm{MeV}$ is real. The sharp decline of the calculated spectrum above $15 \mathrm{MeV}$ might be connected with the limited total excitation en-
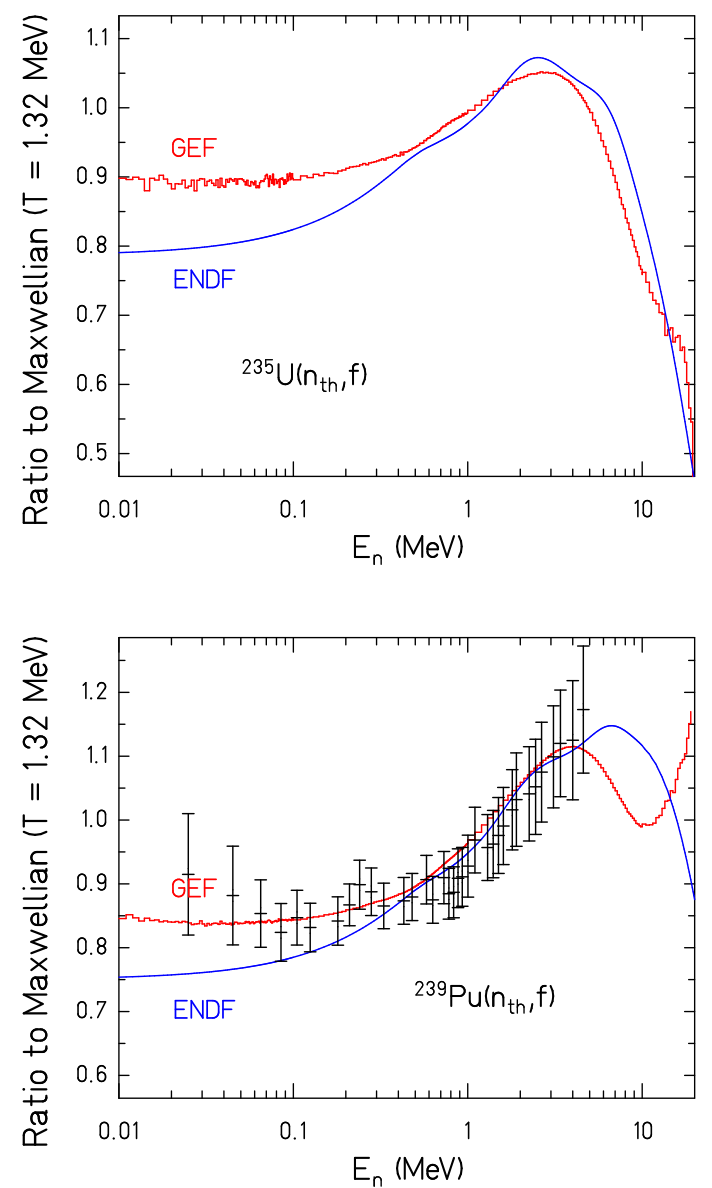

FIG. 84. (Color online) The prompt-neutron energy spectra for the systems ${ }^{235} \mathrm{U}\left(\mathrm{n}_{\mathrm{th}}, \mathrm{f}\right)$ (upper part) and ${ }^{239} \mathrm{Pu}\left(\mathrm{n}_{\mathrm{th}}, \mathrm{f}\right)$ (lower part) resulting from the GEF model (red histogram) are compared with the ENDF/B-VII.1 evaluation 179] (blue line) on a logarithmic energy scale. For the system ${ }^{239} \mathrm{Pu}\left(\mathrm{n}_{\mathrm{th}}, \mathrm{f}\right)$, the experimental data from ref. 227] are shown again in addition (black symbols). The binning of the calculated spectrum is adapted to the number of events in different energy regions (see text). The fluctuations in the calculated spectra below $0.1 \mathrm{MeV}$ and above $10 \mathrm{MeV}$ can be explained by statistical fluctuations of the Monte-Carlo calculation with $10^{9}$ events per system.

TABLE XIII. Bin-size values used in Fig. 84

\begin{tabular}{c|c}
\hline \hline Energy range & Bin size \\
\hline $0.01-0.1 \mathrm{MeV}$ & $1 \mathrm{keV}$ \\
\hline $0.1-1 \mathrm{MeV}$ & $10 \mathrm{keV}$ \\
\hline $1-5 \mathrm{MeV}$ & $100 \mathrm{keV}$ \\
\hline $5-10 \mathrm{MeV}$ & $200 \mathrm{keV}$ \\
\hline $10-20 \mathrm{MeV}$ & $500 \mathrm{keV}$ \\
\hline \hline
\end{tabular}


ergy of the fragments. The differences between the GEF result and the ENDF/B-VII.1 evaluation are similar for the system ${ }^{239} \mathrm{Pu}\left(\mathrm{n}_{\mathrm{th}}, \mathrm{f}\right)$ in the low-energy range, but appreciably stronger at outgoing-neutron energies above 5 $\mathrm{MeV}$, where, unfortunately, no experimental data exist. The experimental data are only shown for the system ${ }^{239} \mathrm{Pu}\left(\mathrm{n}_{\text {th }}, \mathrm{f}\right)$, because the many data for ${ }^{235} \mathrm{U}\left(\mathrm{n}_{\mathrm{th}} \mathrm{f}\right)$ would overload the figure. However, the good agreement between experiment and GEF result can be deduced from Fig. 81. In both cases, the GEF results agree better with the data than the spectra from the ENDF/B-VII.1 evaluation.

In general, the GEF code reproduces the available experimental prompt-fission-neutron energy spectra rather well. This qualifies the GEF code for estimating promptneutron energy spectra in cases where experimental data do not exist. These data can be generated by downloading the code [75] and by performing the calculations for the appropriate fissioning system. The code also seems to be a suitable tool for improving evaluations. Sizable deviations from the prompt-neutron energy spectra of the ENDF/B-VII.1 evaluation are observed in regions where there are scarce or no data.

b. Correlations: Since the prompt-neutron energy spectra measured in the laboratory frame are the result of a convolution due to the emission under different angles from the moving fragments, they are not very sensitive to the yield of neutrons with very low energies in the frame of the fragments. Therefore, one may look for other experimental signatures that are more sensitive to specific features of the neutron emission. One of these signatures is the variation of the neutron multiplicity as a function of the angle between the directions of the emitted neutrons and the light fission fragment. Fig. 85 shows the experimental data 230] in comparison with the result of the GEF code. The measured data are well reproduced over almost the complete angular range. The code un-

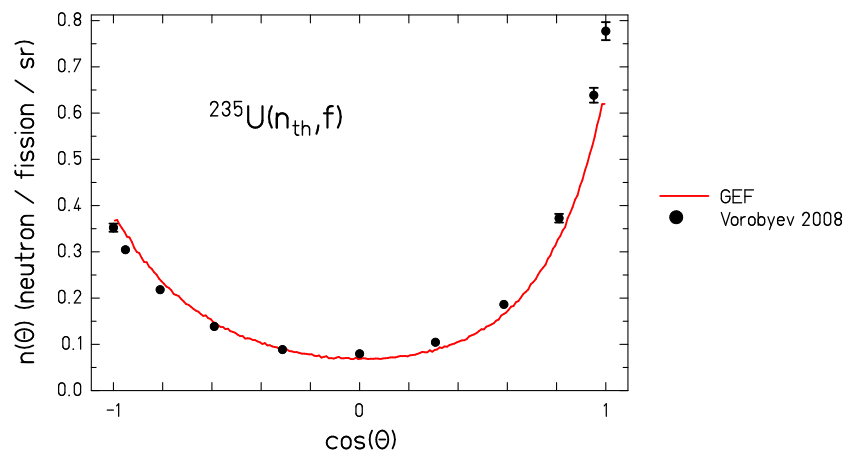

FIG. 85. (Color online) Variation of the prompt-neutron multiplicities versus the neutron direction relative to the direction of the light fission fragment. The result of the GEF code is compared with experimental data from Ref. [230]. The nominal threshold in the experiment was 0.15 to $0.2 \mathrm{MeV}$.
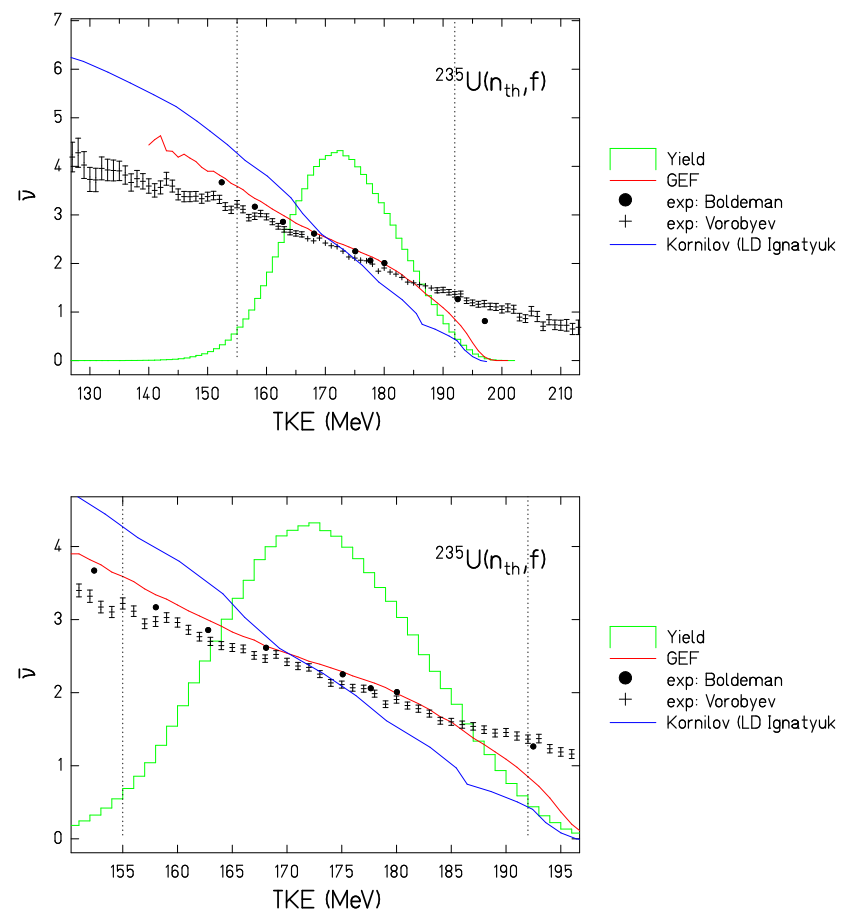

FIG. 86. (Color online) Mean prompt-neutron yield as a function of fission-fragment total kinetic energy for the system ${ }^{235} \mathrm{U}\left(\mathrm{n}_{\mathrm{th}}, \mathrm{f}\right)$. The experimental data of Boldeman et al. 231. and Vorobyev et al. 232] are compared with a calculation of Kornilov 233] (labelled as LD Ignatyuk) and the result of the GEF model (red line). The lower part shows a zoom on the central part of the TKE distribution. The green histogram shows the calculated pre-neutron TKE distribution in an arbitrary scale. The dotted vertical lines denote the region that contains $95 \%$ of the fission events.

derestimates the yield only very close to the direction of the light fragments. The two right-most points of the distribution correspond to angles of 5.7 and 9.9 degrees, corresponding to neutron energies in the fragment frame of 30 and $10 \mathrm{keV}$, respectively. Thus, these deviations can be explained by a slight underestimation of the neutronabsorption cross-sections in the very restricted low-energy regime below $50 \mathrm{keV}$. In the prompt-neutron energy spectrum, Figs. 81 to 83 , these events appear at laboratory energies around $1 \mathrm{MeV}$ due to the velocity of the emitting fragment. Here, no indication for this deviation can be seen. It seems that the description by the GEF code is very well suited for estimating the prompt-neutron energy spectra in the laboratory frame of heavy fissioning systems, which are most important for technical applications. The slight deviations in the angular distributions, Fig. 85, have practically no influence on the energy distribution of the prompt neutrons in the laboratory frame.

In the following, we investigate the prompt-neutron yield as a function of the fission-fragment total kinetic energy. Fig. 86 shows a comparison of the result of the 


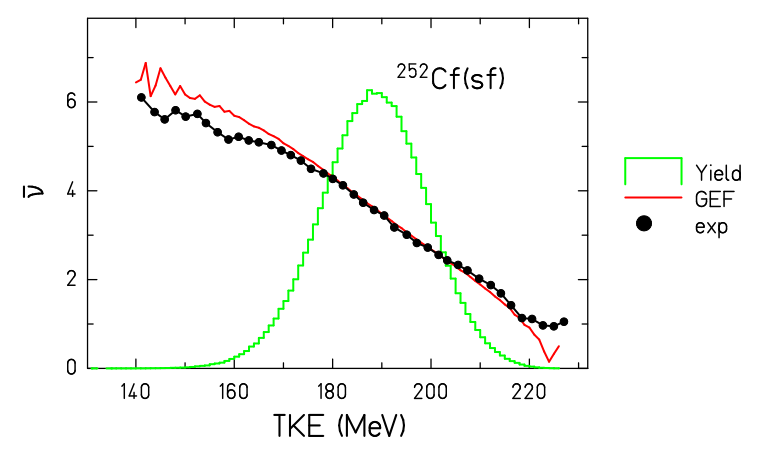

FIG. 87. (Color online) Mean prompt-neutron yield as a function of fission-fragment total kinetic energy for the system ${ }^{252} \mathrm{Cf}(\mathrm{sf})$. The experimental data of Budtz-Jorgensen et al. 216] are compared with the result of the GEF model. The green histogram shows the calculated pre-neutron TKE distribution in an arbitrary scale.

GEF code with experimental data 231, 232 and a previous calculation of Kornilov [233]. The GEF calculation has been performed using Thomas-Fermi masses of Myers and Swiatecki [18] with recommended shell corrections and schematic even-odd fluctuations. The variation of the prompt-neutron yields from the light and the heavy fragment are assumed to be uncorrelated for a given split in $Z$ and $N$.

The GEF calculation, in particular the slope, is rather close to the experimental data in the region between 155 $\mathrm{MeV}$ and $185 \mathrm{MeV}$. Also, the low-energy point of Boldeman et al. is well reproduced. For energies higher than $185 \mathrm{MeV}$, all calculations, also the calculation of Kornilov, are appreciably below the experimental data. The cut-off of the neutron multiplicity slightly below $200 \mathrm{MeV}$ is probably realistic, because even for the splits with the highest $Q$ values the excitation energies of the fragments fall below the corresponding neutron separation energy for these high TKE values.

One should not forget that scattering phenomena can considerably disturb experimental data in regions of low yield as e.g. demonstrated in Ref. 234]. Such processes would tend to flatten the variation of the measured prompt-neutron yield as a function of TKE. In this context, it is interesting to note that the data of Boldeman et al. have a steeper slope than the data of Vorobyev et al., especially in the wings of the TKE distribution. The data of Vorobyev et al. even extend to TKE values, where there is hardly any yield expected, and neutrons are still seen above TKE $=200 \mathrm{MeV}$, where neutron emission is suppressed in the GEF code due to the Q-value limit. This puts also doubts on the data of Vorobyev et al. for total kinetic energies below $150 \mathrm{MeV}$, where the yield is low, and scattering phenomena may have an important influence.

The GEF code reproduces also well the measured mean prompt-neutron yields as a function of the total fission- fragment kinetic energy for spontaneous fission of ${ }^{252} \mathrm{Cf}$ of Ref. 216], see Fig. 87. The deviations at high TKE appear in a region of extremely low yield. They may be explained by a background of events with lower TKE due to random coincidences of fragment and prompt-neutron signals in the experiment. Also the deviations at low TKE appear in a region with low yield. They may be caused at least to a part by incompletely measured TKE values due to scattering phenomena in the experiment.

One may speculate that the transport of a multitude of correlations along the fission process in the GEF code without any intermediate averaging has an important influence on correlations between different fission observables. These correlations might not have been fully considered in other models. The calculations with the GEF code do not give strong hints for additional phenomena like scission neutrons; the data of Figs. 86 and 87 can rather well be reproduced with the assumption of promptneutron emission from the fragments after scission, only.

c. Conclusion: The GEF model reproduces a large variety of neutron observables with a good precision in a consistent way without further adjustment to specific fissioning systems with a unique parameter set. With this global approach one is able to predict several characteristic quantities of the fission process, e.g. the energy and multiplicity distribution of prompt-fission neutrons, without the need for specific experimental information of the respective system, e.g. measured mass-TKE distributions. We recall that all properties of the fission fragments that are considered in the code (e.g. nuclear charge, mass, excitation energy, angular momentum) are sampled in the corresponding multi-dimensional parameter space by a Monte-Carlo technique (see Sec. VIII). Thus, all respective correlations are preserved. Moreover, correlations between all observables considered in the code are provided on an event-by-event basis. It should be stressed that it is straightforward to deduce covariances for the calculated prompt-neutron energy spectrum determined by the inner logic of the GEF model in analogy to the covariances of the fission-fragment yields from the list-mode data of the perturbed-parameter calculations.

The measured prompt-neutron energy spectra in fission induced by thermal neutrons are very well reproduced by the GEF code without any specific adjustment of the model for all systems that were investigated. It is to be expected that this agreement is preserved for fission induced by neutrons of higher energies. There are no systematic deviations which suggest the presence of scission neutrons in these cases.

\section{Energy Dependence}

a. Introduction: This section deals with the description of prompt-neutron spectra in neutron-induced fission reactions over a larger excitation-energy range extending from spontaneous fission to multi-chance fission. A 
number of measured prompt-neutron spectra from elaborate experiments are compared with the results of the GEF code 75, 165]. The GEF code calculates the contributions from the excited nucleus before scission and from the fragments simultaneously with the statistical model in a consistent way together with many other fission observables. The calculation is done without using an analytical formula with adjustable parameters for the shape of the prompt-neutron spectrum and without any input on fission-fragment properties for specific systems. Therefore, this study aims to give a coherent picture on the variation of the prompt-neutron spectrum for different fissioning systems as a function of excitation energy.

b. Description of the Calculation: The following figures show comparisons of measured fission promptneutron spectra extracted from EXFOR with results of the GEF code [75, 165]. All measurements have been performed relative to the system ${ }^{252} \mathrm{Cf}(\mathrm{sf})$. Thus, the data marked as ratio or $\mathrm{R}$ are directly measured. If the deduced prompt-neutron spectra are also given in EXFOR, they are shown as well, marked as yield or Y. The scale is $\mathrm{d} N / \mathrm{d} E$ in units of $1 / \mathrm{MeV}$.

GEF calculations on neutron yields and energy distributions have been performed for the indicated systems and for ${ }^{252} \mathrm{Cf}(\mathrm{sf})$. All calculations have been performed without any adjustment to specific systems with the very same parameter set. No particular information from experimental data, e.g. A-TKE spectra, has been used.

In order to clearly distinguish the calculation of prompt-neutron yields with the general approach of the GEF model from other models, a short summary of alternative approaches seems to be appropriate. One of the first widespread descriptions of the prompt-neutron spectrum was introduced by Watt 235. He proposed a closed formula, deduced from a Maxwell-type energy spectrum from one or two average fragments and the transformation into the frame of the fissioning system with at least two adjustable parameters: the temperature and the velocity of the average fragment. The "Los-Alamos model" [236] extended this approach essentially by the use of a triangular temperature distribution of the fragments to a four-term closed expression for an average light and an average heavy fragment. A similar two-fragment model was also used by Kornilov et al. in [223]. In 1989, Madland et al. 237] introduced the point-by-point model by considering the emission from all individual fragments, specified by $Z$ and $A$. This model was further developed e.g. by Lemaire et al. [238], Tudora et al. [239] and Vogt et al. [240]. In Refs. 241 244], the spectral shape was parameterized by the Watt formula 235] or an empirical shape function that had been introduced by Mannhart 245] in order to better model the shape of the neutron energy spectra in the fragment frame. Kornilov [246] proposed a phenomenological approach for the parameterization of a model-independent shape of the prompt-neutron spectrum. This approach was later also used by Kodeli et al. 247] and Maslov et al. 248]. These models often reach a high degree of agreement with the measured prompt-neutron spectra for particular fissioning systems with especially adjusted parameters. All models cited above are based on empirical data: the Watt model and the Los-Alamos model are directly fitted to the measured prompt-neutron spectrum, while the point-bypoint model is based on the measured $A$-TKE distribution. Manea et al. 249] proposed a scission-point model that predicts the $\operatorname{TKE}(A)$ distribution, in order to allow for calculations of prompt-neutron spectra with the point-by-point method if only the mass distribution is known. For completeness, we also mention a paper of Howerton [250], who developed a systematics for calculating the contributions of the post-fission neutrons to the total prompt-neutron spectra from all $(\mathrm{n}, \mathrm{xnf})$ reactions. This systematics was used in [251].

As a result of the GEF model, the prompt-neutron spectra and the ratios to the calculated ${ }^{252} \mathrm{Cf}(\mathrm{sf})$ spectrum are shown in Figs. 88 to 114 . Note that the measured and the calculated spectrum for ${ }^{252} \mathrm{Cf}(\mathrm{sf})$ are shown in Fig. 81. Due to the Monte-Carlo method used in the GEF code, the calculated spectra show statistical fluctuations, especially in the high-energy tail. The calculated total prompt-neutron multiplicity is given in addition in the figures. While the data are drawn in black, the colored lines denote the results of the GEF model for the neutron yields $(Y)$ and the neutron-yield ratios $(R)$ to the case of ${ }^{252} \mathrm{Cf}(\mathrm{sf})$ as explained in the figures. Note that the deviations between GEF results and experimental data in the two representations (ratio and yield) are not consistent, because the GEF yield ratios and the experimental yields (measured yield ratios times neutron yields for ${ }^{252} \mathrm{Cf}(\mathrm{sf})$ ) have been obtained with different prompt-neutron reference spectra: For the GEF ratios the calculated ${ }^{252} \mathrm{Cf}(\mathrm{sf})$ spectrum was used, for the experimental yields an evaluated ${ }^{252} \mathrm{Cf}(\mathrm{sf})$ spectrum was used. It seems that most of the experiments aimed only to determine the shape of the spectra. Therefore, an arbitrary scaling factor was applied to the measured spectra, such that the total prompt-neutron multiplicity agrees approximately with the GEF result. These scaling factors are listed in the legends of the figures. All figures are shown in linear and logarithmic scale.

\section{c. Results:}

1. Spectra:

${ }^{232} \mathbf{T h}(\mathbf{n}, \mathbf{f}), E_{n}=\mathbf{2 . 9} \mathbf{M e V}$ : The measured spectrum is very well reproduced up to $7 \mathrm{MeV}$. At higher energies, the measured spectrum shows strange fluctuations, which points at experimental uncertainties.

${ }^{232} \mathbf{T h}(\mathbf{n}, \mathbf{f}), E_{n}=\mathbf{1 4 . 7} \mathrm{MeV}$ : Most part of the spectrum is very well reproduced by the calculation. However, there is a local enhancement at very low energies, which is not strong enough in the calculation below $0.5 \mathrm{MeV}$. The structure around $8 \mathrm{MeV}$ is narrower and slightly shifted in the calculation. 

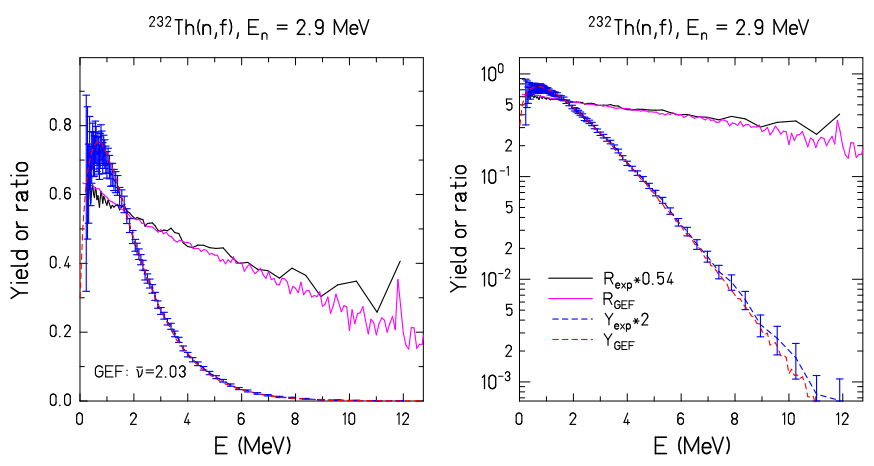

FIG. 88. (Color online) Prompt-neutron spectrum from ${ }^{232} \mathrm{Th}(\mathrm{n}, \mathrm{f}), E_{n}=2.9 \mathrm{MeV}$. (Data from EXFOR dataset 411100081.)
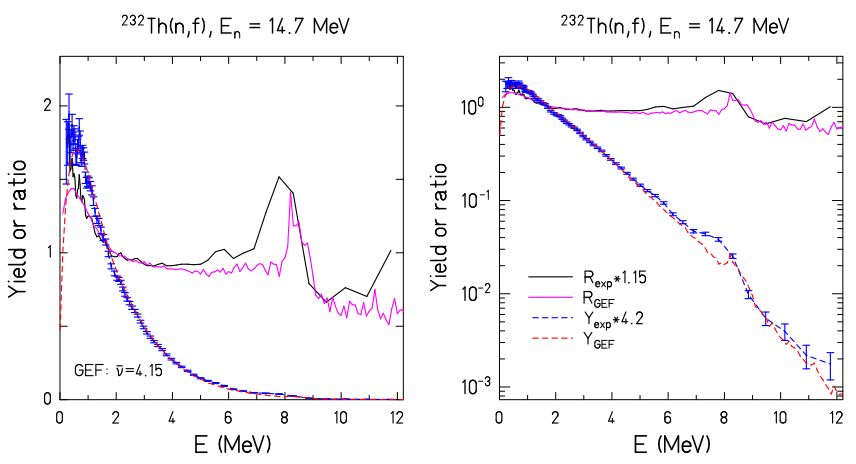

FIG. 89. (Color online) Prompt-neutron spectrum from ${ }^{232} \mathrm{Th}(\mathrm{n}, \mathrm{f}), E_{n}=14.7 \mathrm{MeV}$. (Data from EXFOR dataset 411100081.)
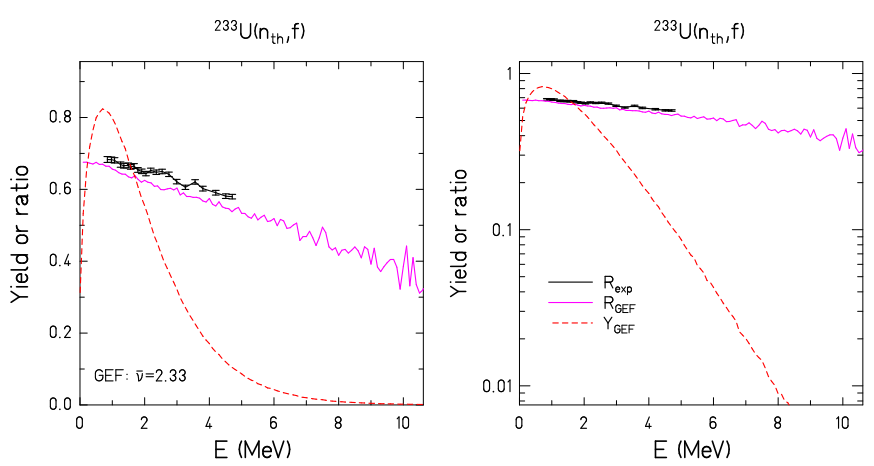

FIG. 90. (Color online) Prompt-neutron spectrum from ${ }^{233} \mathrm{U}\left(\mathrm{n}_{\mathrm{th}}, \mathrm{f}\right)$. (Data from EXFOR dataset 40871013.)

${ }^{233} \mathbf{U}\left(\mathbf{n}_{\mathrm{th}}, \mathbf{f}\right)$ : The calculated spectrum is very well reproduced in the range between 0.8 and $4.7 \mathrm{MeV}$ that is covered by the experiment. (The spectrum was not normalised.)
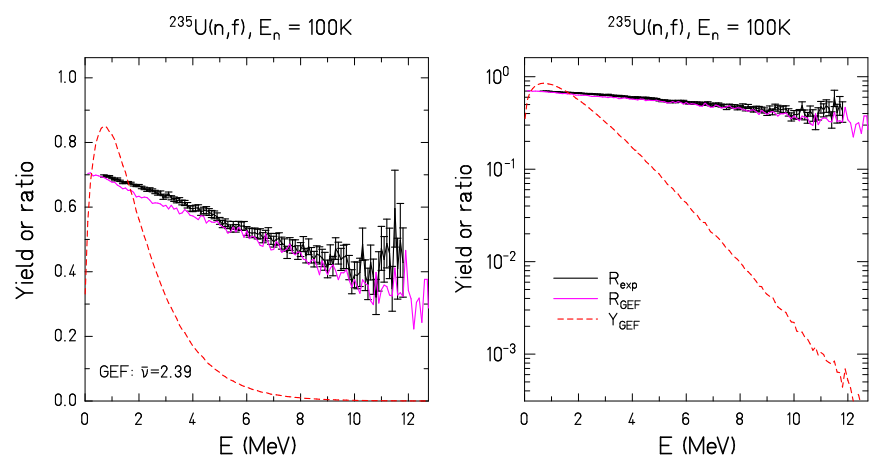

FIG. 91. (Color online) Prompt-neutron spectrum from ${ }^{235} \mathrm{U}(\mathrm{n}, \mathrm{f}), \quad E_{n}=100 \mathrm{~K}$. (Data from EXFOR dataset 31692006.)
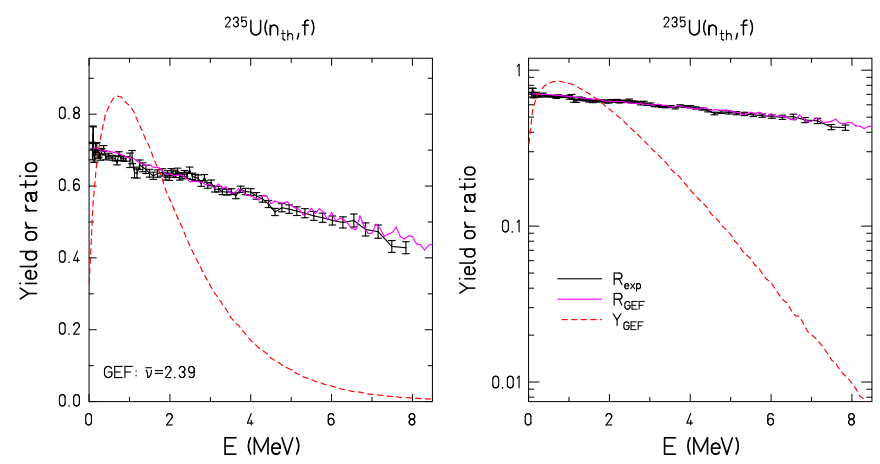

FIG. 92. (Color online) Prompt-neutron spectrum from ${ }^{235} \mathrm{U}\left(\mathrm{n}_{\mathrm{th}}, \mathrm{f}\right)$. (Data from EXFOR datasets 40871011, 40871012.)
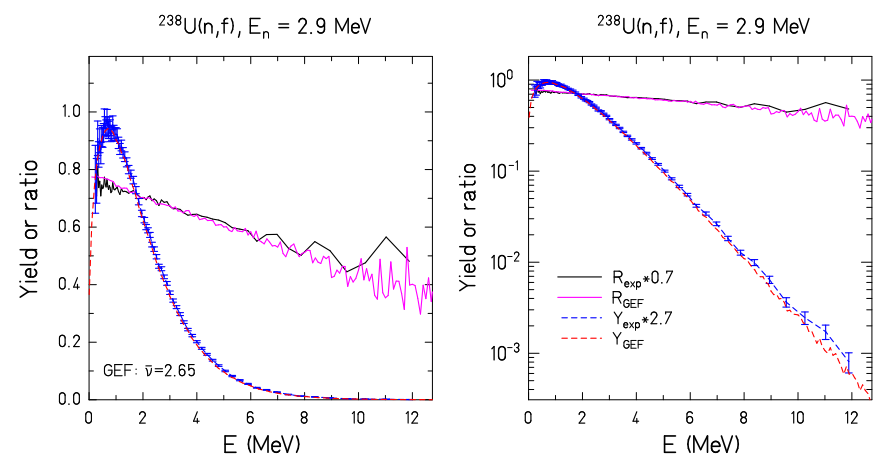

FIG. 93. (Color online) Prompt-neutron spectrum from ${ }^{238} \mathrm{U}(\mathrm{n}, \mathrm{f}), \quad E_{n}=2.9 \mathrm{MeV}$. (Data from EXFOR dataset 411100101.)

${ }^{235} \mathbf{U}(\mathbf{n}, \mathbf{f}), E_{n}=\mathbf{1 0 0} \mathbf{K}$ : The spectrum is well reproduced over the whole energy range. Between $1 \mathrm{MeV}$ and $5 \mathrm{MeV}$, the calculated spectrum is a little bit lower. (The spectrum was not normalized.) 

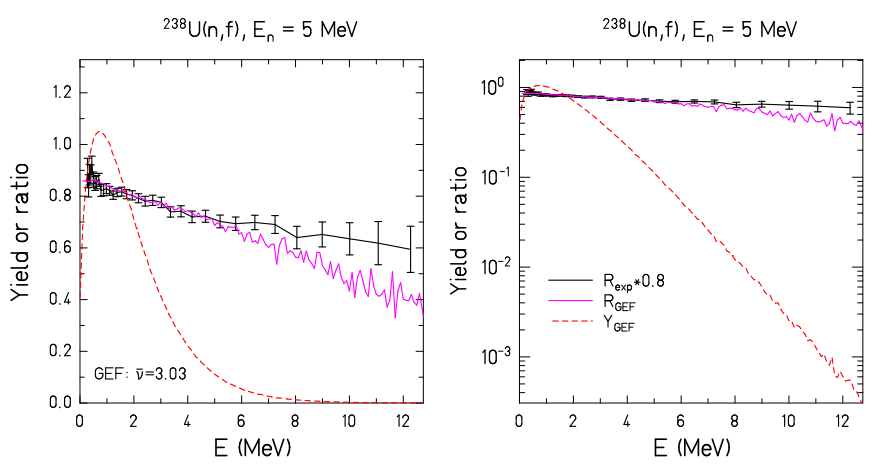

FIG. 94. (Color online) Prompt-neutron spectrum from ${ }^{238} \mathrm{U}(\mathrm{n}, \mathrm{f}), \quad E_{n}=5 \mathrm{MeV}$. (Data from EXFOR dataset 41450003.)
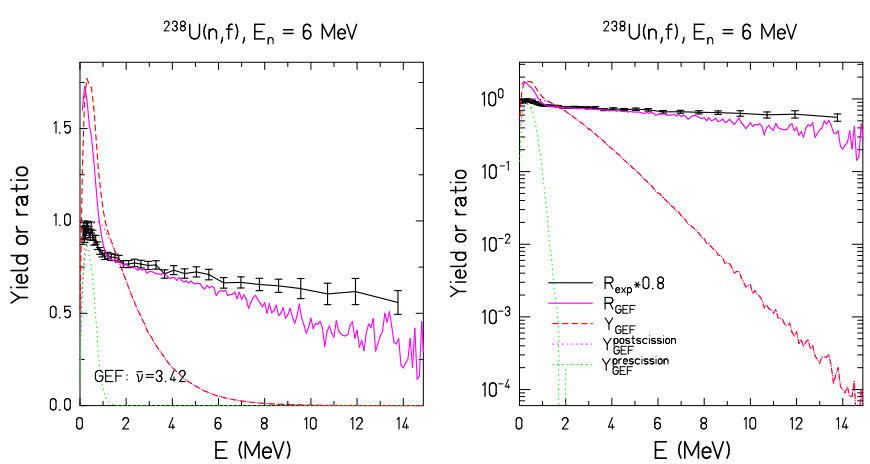

FIG. 95. (Color online) Prompt-neutron spectrum from ${ }^{238} \mathrm{U}(\mathrm{n}, \mathrm{f}), \quad E_{n}=6 \mathrm{MeV}$. (Data from EXFOR dataset 41447003.)
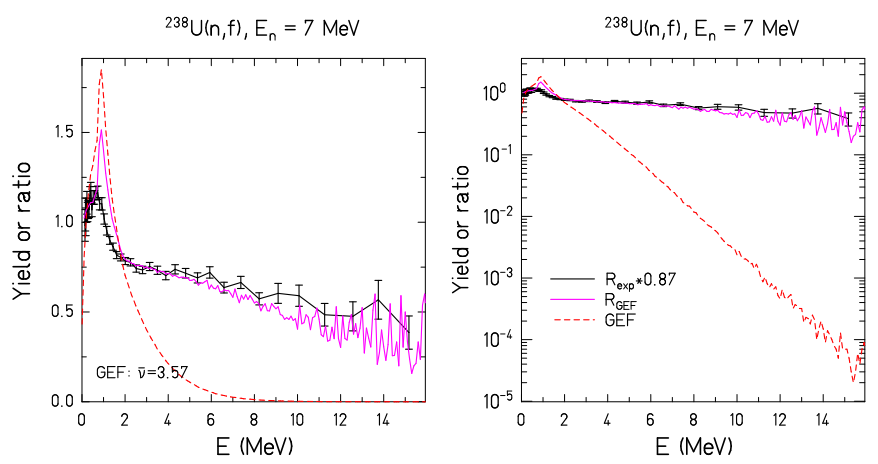

FIG. 96. (Color online) Prompt-neutron spectrum from ${ }^{238} \mathrm{U}(\mathrm{n}, \mathrm{f}), \quad E_{n}=7 \mathrm{MeV}$. (Data from EXFOR dataset 41447003.)

${ }^{235} \mathbf{U}\left(\mathbf{n}_{\mathrm{th}}, \mathbf{f}\right)$ : The measured spectrum is very well reproduced over the whole energy range.

${ }^{238} \mathbf{U}(\mathbf{n}, \mathbf{f}), E_{n}=\mathbf{2 . 9} \mathrm{MeV}$ : The measured spectrum is very well reproduced in the energy range below $6 \mathrm{MeV}$.
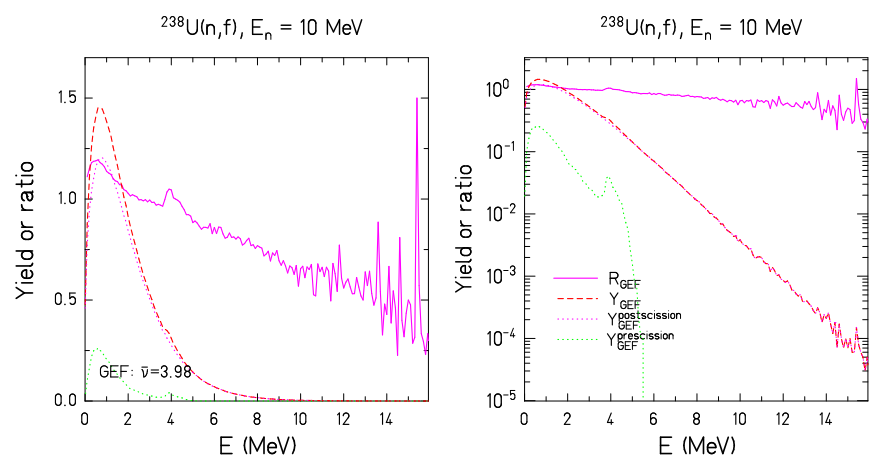

FIG. 97. (Color online) Prompt-neutron spectrum from ${ }^{238} \mathrm{U}(\mathrm{n}, \mathrm{f}), E_{n}=10 \mathrm{MeV}$. (No data available.)
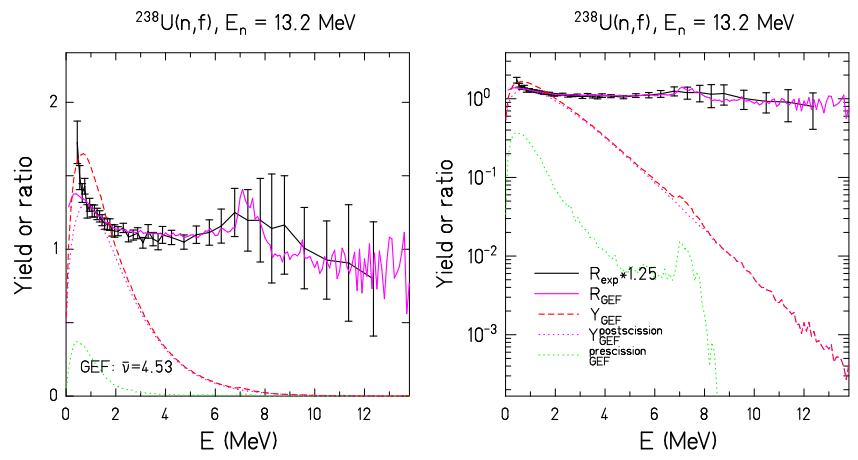

FIG. 98. (Color online) Prompt-neutron spectrum from ${ }^{238} \mathrm{U}(\mathrm{n}, \mathrm{f}), E_{n}=13.2 \mathrm{MeV}$. (Data from EXFOR dataset 41450003.)
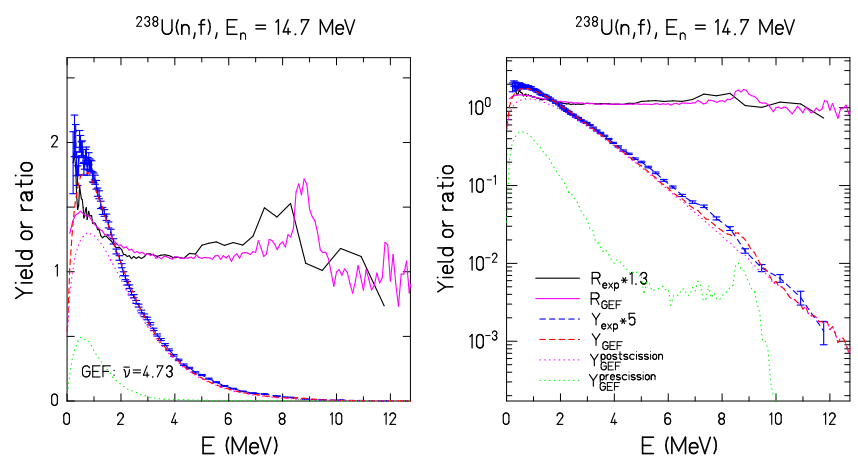

FIG. 99. (Color online) Prompt-neutron spectrum from ${ }^{238} \mathrm{U}(\mathrm{n}, \mathrm{f}), E_{n}=14.7 \mathrm{MeV}$. (Data from EXFOR dataset 411100101.)

At higher energies, the measured spectrum shows strange fluctuations, which points at experimental uncertainties.

${ }^{238} \mathbf{U}(\mathbf{n}, \mathbf{f}), E_{n}=\mathbf{5} \mathbf{M e V}$ : The measured spectrum is very well reproduced up to $5 \mathrm{MeV}$. At $5 \mathrm{MeV}$ there is a kink in the measured data, and the data have a smaller 

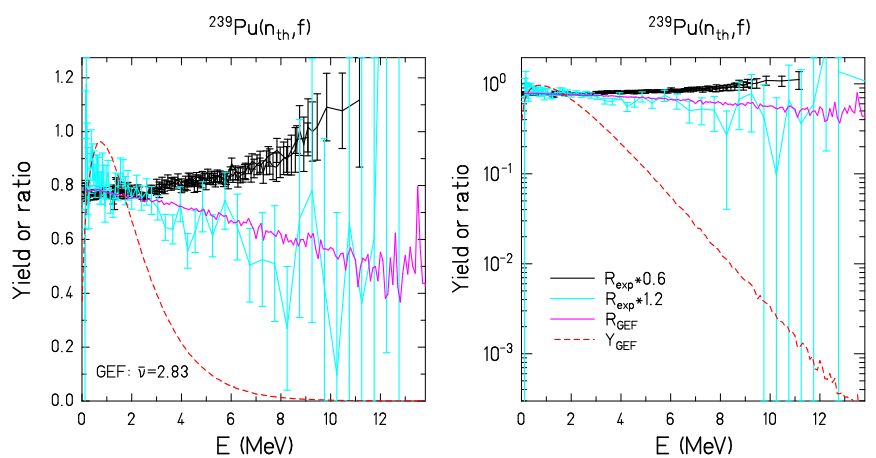

FIG. 100. (Color online) Prompt-neutron spectrum from ${ }^{239} \mathrm{Pu}\left(\mathrm{n}_{\mathrm{th}}, \mathrm{f}\right)$. (Data from EXFOR datasets 40871009, 40871010, 40872006, 41502004.)
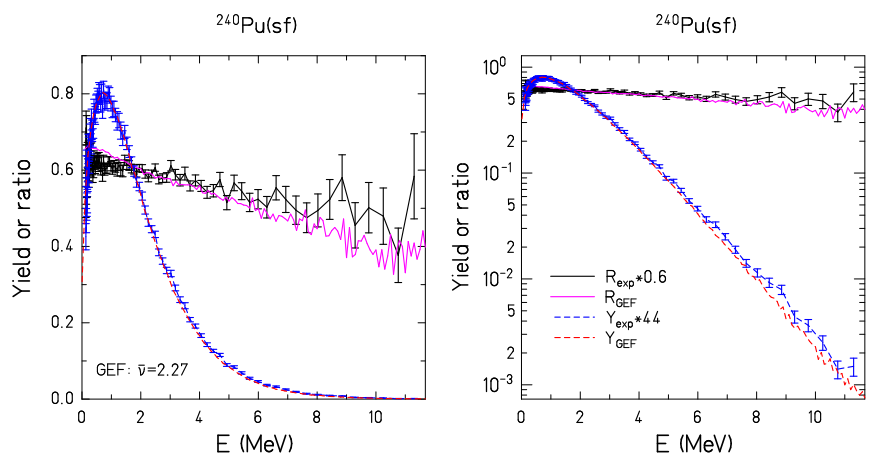

FIG. 101. (Color online) Prompt-neutron spectrum from ${ }^{240} \mathrm{Pu}(\mathrm{sf})$. (Data from EXFOR dataset 414210021.)
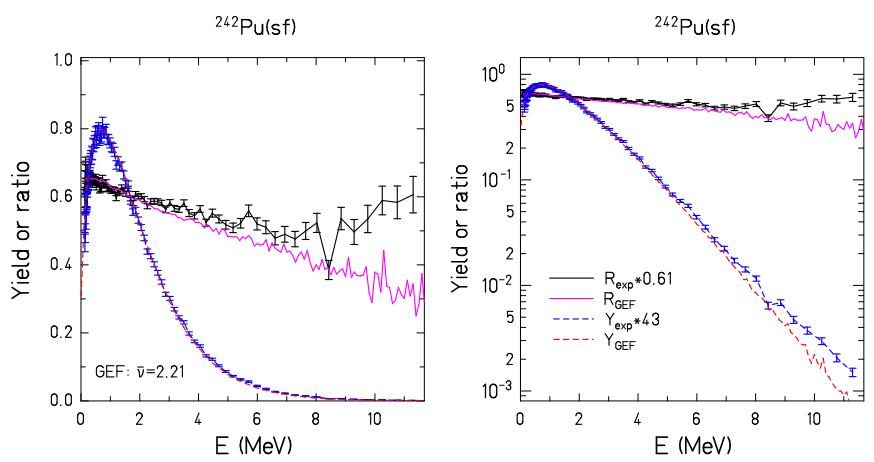

FIG. 102. (Color online) Prompt-neutron spectrum from ${ }^{242} \mathrm{Pu}(\mathrm{sf})$. (Data from EXFOR dataset 414210031.)

slope at higher energies. This may point at a background component in the experiment.

${ }^{238} \mathrm{U}(\mathbf{n}, \mathbf{f}), E_{n}=\mathbf{6} \mathrm{MeV}$ : The calculated spectrum above $1 \mathrm{MeV}$ has a steeper slope than the measured one. In addition, the calculated spectrum is enhanced at the lowest energies due to a contribution from second-chance
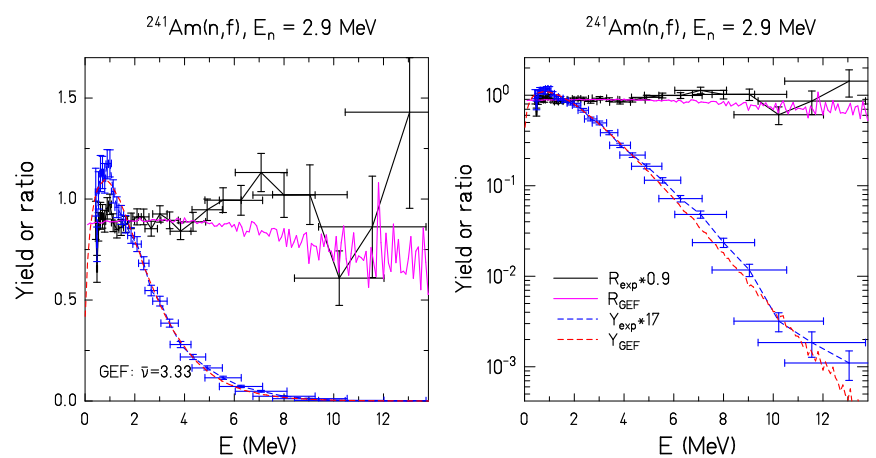

FIG. 103. (Color online) Prompt-neutron spectrum from ${ }^{241} \mathrm{Am}(\mathrm{n}, \mathrm{f}), E_{n}=2.9 \mathrm{MeV}$. (Data from EXFOR dataset 415890021.)
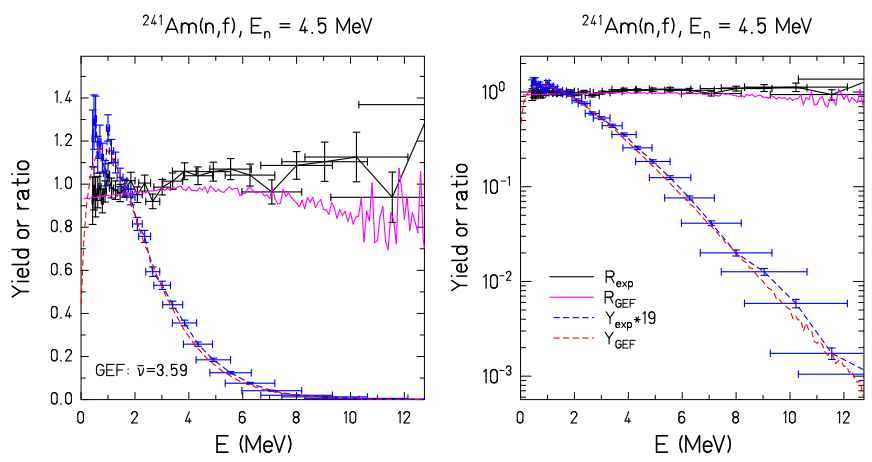

FIG. 104. (Color online) Prompt-neutron spectrum from ${ }^{241} \mathrm{Am}(\mathrm{n}, \mathrm{f}), E_{n}=4.5 \mathrm{MeV}$. (Data from EXFOR dataset 415890031.)
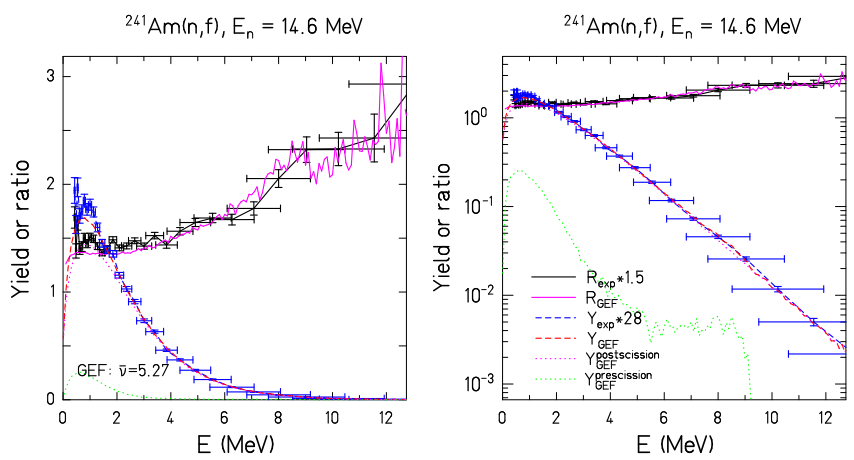

FIG. 105. (Color online) Prompt-neutron spectrum from ${ }^{241} \mathrm{Am}(\mathrm{n}, \mathrm{f}), E_{n}=14.6 \mathrm{MeV}$. (Data from EXFOR dataset 415890041.)

fission. This enhancement is overestimated by the calculation.

${ }^{238} \mathrm{U}(\mathbf{n}, \mathbf{f}), \quad E_{n}=\mathbf{7} \mathrm{MeV}$ : The calculated spectrum above $1 \mathrm{MeV}$ has a slightly steeper slope than the mea- 

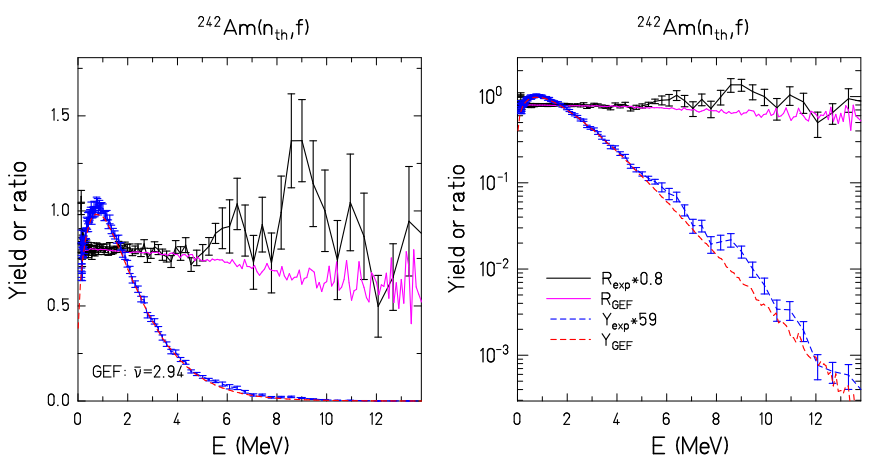

FIG. 106. (Color online) Prompt-neutron spectrum from ${ }^{242} \mathrm{Am}\left(\mathrm{n}_{\mathrm{th}}, \mathrm{f}\right)$. (Data from EXFOR dataset 414210081 .)
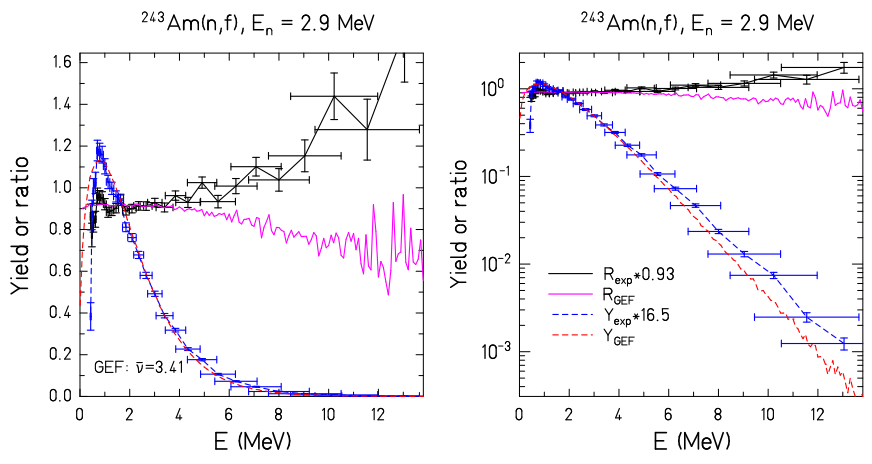

FIG. 107. (Color online) Prompt-neutron spectrum from ${ }^{243} \mathrm{Am}(\mathrm{n}, \mathrm{f}), E_{n}=2.9 \mathrm{MeV}$. (Data from EXFOR dataset 415890051.)

sured one. The spectrum is enhanced at the lowest energies due to a contribution from second-chance fission. Amplitude, width and position of this structure are not correctly reproduced by the calculation.

${ }^{238} \mathbf{U}(\mathbf{n}, \mathbf{f}), E_{n}=\mathbf{1 0} \mathbf{M e V}$ : This spectrum, for which no data are available, is added in order to allow a systematic view on the variation of the structure caused by the threshold of second- chance fission.

${ }^{238} \mathrm{U}(\mathbf{n}, \mathbf{f}), E_{n}=\mathbf{1 3 . 2} \mathrm{MeV}$ : The spectrum is well reproduced by the model within the experimental uncertainties. However, there is a local enhancement at very low energies, which is not strong enough in the calculation. The structure due to the threshold of second-chance fission is slightly shifted to lower energies and narrower in the calculation.

${ }^{238} \mathrm{U}(\mathbf{n}, \mathbf{f}), E_{n}=\mathbf{1 4 . 7} \mathrm{MeV}$ : Again, there is a local enhancement at very low energies below $0.6 \mathrm{MeV}$, which is not strong enough in the calculation. The shape and the position of the structure due to the threshold of secondchance fission are not correctly reproduced by the calculation.

${ }^{239} \mathbf{P u}\left(\mathbf{n}_{\mathrm{th}}, \mathbf{f}\right)$ : There are two experimental results with different slopes of the high-energy tail. The slope of the
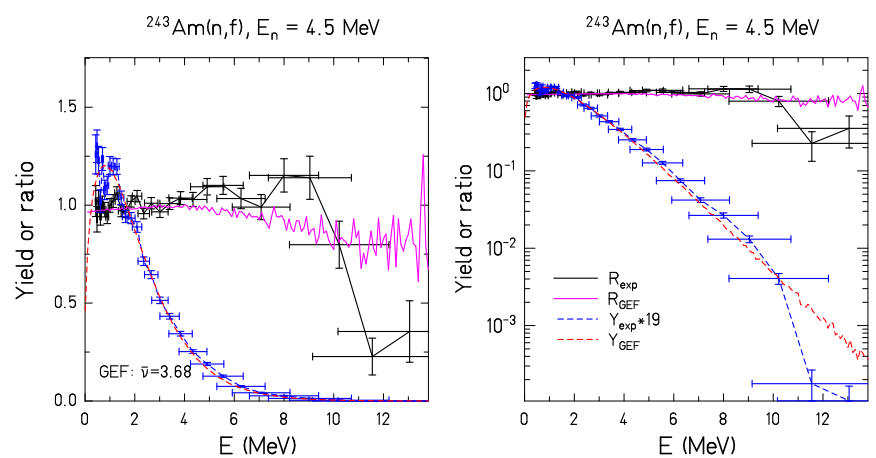

FIG. 108. (Color online) Prompt-neutron spectrum from ${ }^{243} \mathrm{Am}(\mathrm{n}, \mathrm{f}), E_{n}=4.5 \mathrm{MeV}$. (Data from EXFOR dataset 415890061.)
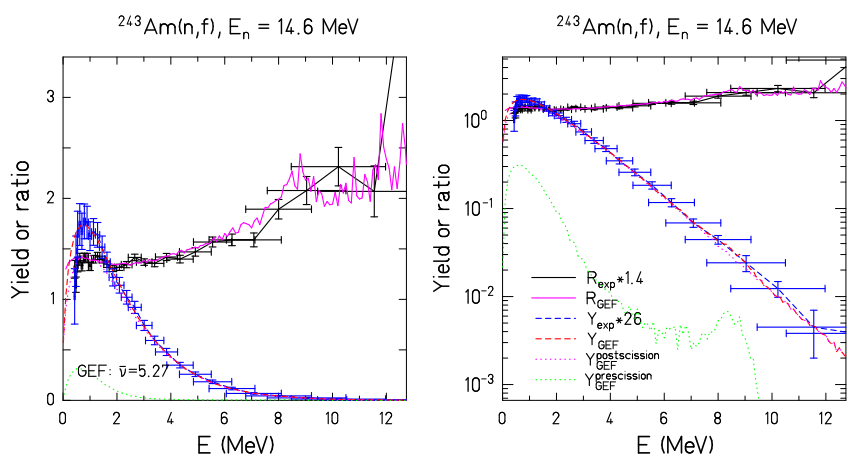

FIG. 109. (Color online) Prompt-neutron spectrum from ${ }^{243} \mathrm{Am}(\mathrm{n}, \mathrm{f}), E_{n}=14.6 \mathrm{MeV}$. (Data from EXFOR dataset 415890071.)
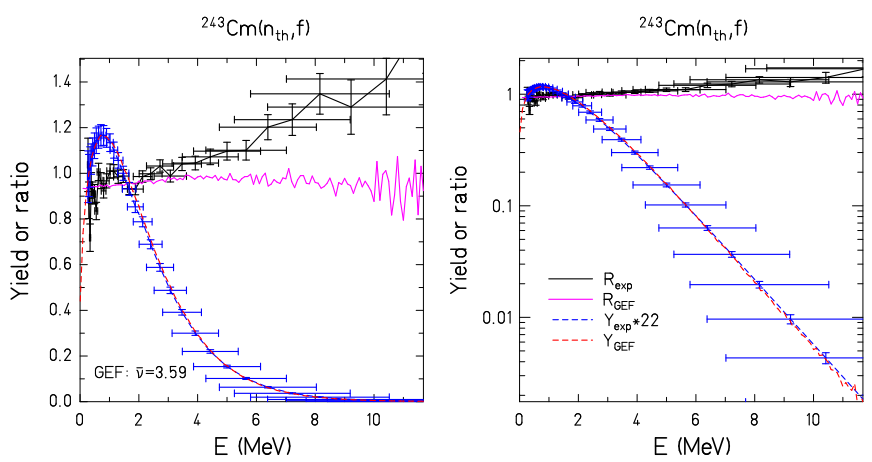

FIG. 110. (Color online) Prompt-neutron spectrum from ${ }^{243} \mathrm{Cm}\left(\mathrm{n}_{\mathrm{th}}, \mathrm{f}\right)$. (Data from EXFOR dataset 415890081.$)$

calculated spectrum agrees better with the steeper slope of one of the experiments, although this spectrum shows strong local fluctuations. The steeper slope is also much closer to the ones of the systems ${ }^{238} \mathrm{U}(\mathrm{n}, \mathrm{f}), E_{n}=2.9 \mathrm{MeV}$ and ${ }^{246} \mathrm{Cm}(\mathrm{sf})$, which have similar total prompt-neutron 

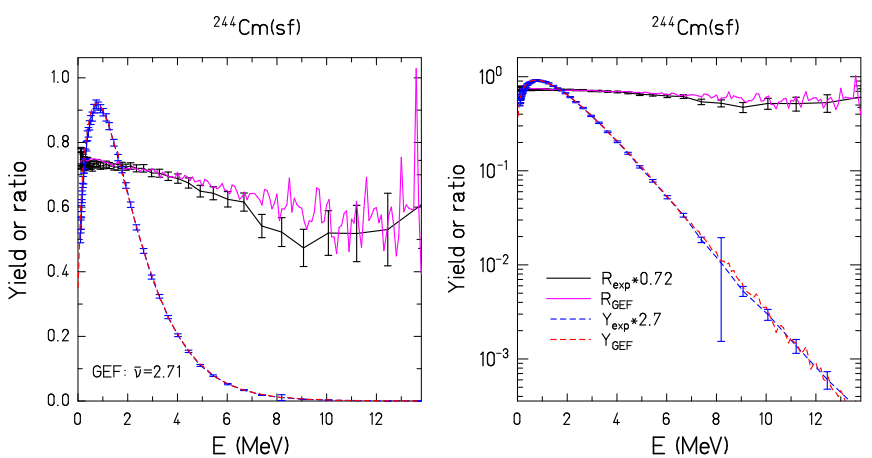

FIG. 111. (Color online) Prompt-neutron spectrum from ${ }^{244} \mathrm{Cm}(\mathrm{sf})$. (Data from EXFOR dataset 413400041 .)
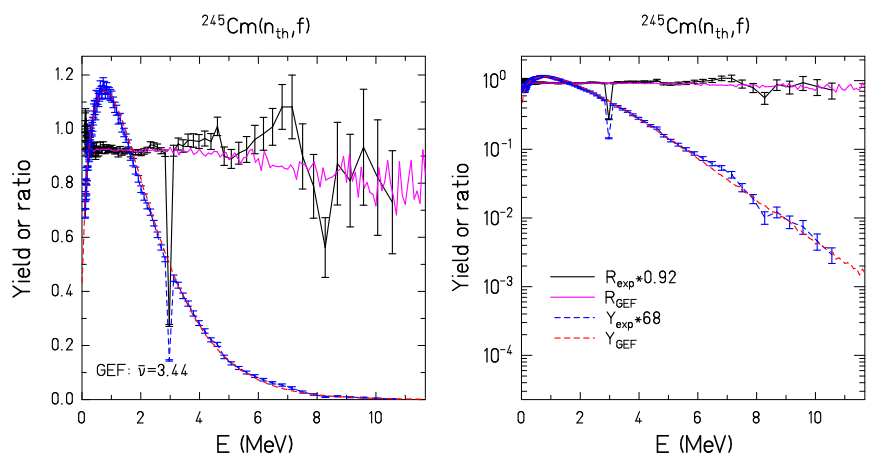

FIG. 112. (Color online) Prompt-neutron spectrum from ${ }^{245} \mathrm{Cm}\left(\mathrm{n}_{\mathrm{th}}, \mathrm{f}\right)$. (Data from EXFOR dataset 414210091.)
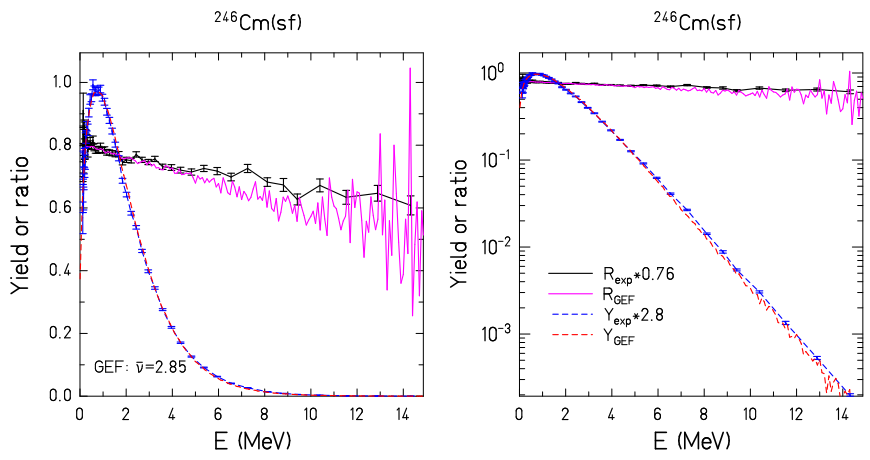

FIG. 113. (Color online) Prompt-neutron spectrum from ${ }^{246} \mathrm{Cm}(\mathrm{sf})$. (Data from EXFOR dataset 413400051.)

yields as ${ }^{239} \mathrm{Pu}(\mathrm{nth}, \mathrm{f})$. Since all these cases are restricted to first-chance fission, one should expect that the total prompt-neutron yield is a measure of the mean excitation energies of the primary fragments, which means that it should be correlated with the slope of the high-energy tail of the prompt-neutron spectrum. Due to this argument, the spectrum with the steeper slope appears to be more
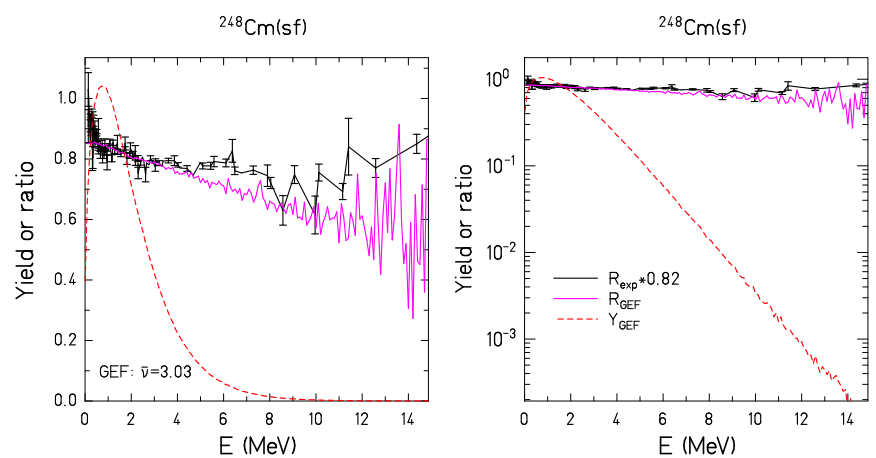

FIG. 114. (Color online) Prompt-neutron spectrum from ${ }^{248} \mathrm{Cm}$ (sf). (Data from EXFOR dataset 41113004.)

likely the correct one.

${ }^{240} \mathbf{P u}(\mathbf{s f}),{ }^{242} \mathbf{P u}(\mathbf{s f})$ : The measured spectra are well reproduced by the calculation, if the fluctuations in the experiment at higher energies are disregarded.

${ }^{241} \mathrm{Am}(\mathbf{n}, \mathbf{f}), E_{n}=\mathbf{2 . 9} \mathrm{MeV}$ : Below $4 \mathrm{MeV}$, the measured spectrum is well reproduced by the calculation. A comparison at higher energies is difficult due to the strong fluctuations in the measured spectrum.

${ }^{241} \operatorname{Am}(\mathbf{n}, \mathbf{f}), E_{n}=4.5 \mathrm{MeV}$ : The measured spectrum is well reproduced by the calculation below $2.5 \mathrm{MeV}$. Above this energy the experimental data fluctuate rather strongly.

${ }^{241} \mathbf{A m}(\mathbf{n}, \mathbf{f}), E_{n}=\mathbf{1 4 . 6} \mathbf{M e V}$ : The measured spectrum is very well reproduced by the calculation, including the structure around $9 \mathrm{MeV}$.

${ }^{242} \mathbf{A m}\left(\mathbf{n}_{\text {th }}, \mathbf{f}\right):$ Below $4.5 \mathrm{MeV}$, the measured spectrum is very well reproduced by the calculation. A comparison is difficult at higher energies due to the strong fluctuations in the measured spectrum.

${ }^{243} \mathrm{Am}(\mathbf{n}, \mathbf{f}), E_{n}=\mathbf{2 . 9} \mathrm{MeV}$ : The measured spectrum is well reproduced below $4 \mathrm{MeV}$. The calculated spectrum is much softer in the high-energy tail than the measured one. It is astonishing that the measured spectrum is appreciably stiffer than the spectrum of ${ }^{252} \mathrm{Cf}(\mathrm{sf})$, although the total prompt neutron yield is almost the same. This points at an experimental problem.

${ }^{243} \operatorname{Am}(\mathbf{n}, \mathbf{f}), E_{n}=4.5 \mathrm{MeV}$ : The measured spectrum is well reproduced below $4 \mathrm{MeV}$. A comparison at higher energies is difficult due to the strong fluctuations of the measured spectrum.

${ }^{243} \operatorname{Am}(\mathbf{n}, \mathbf{f}), E_{n}=14.6 \mathrm{MeV}$ : The measured spectrum is generally well reproduced by the calculation. The structure around $8 \mathrm{MeV}$ is slightly shifted to lower energies.

${ }^{243} \mathbf{C m}\left(\mathbf{n}_{\mathrm{th}}, \mathbf{f}\right)$ : When comparing the measured and the calculated ratios to the spectrum of ${ }^{252} \mathrm{Cf}(\mathrm{sf})$, the calculated spectrum appears to be much softer than the measured one. It is astonishing that the measured spectrum is as stiff as the one for ${ }^{243} \mathrm{Am}(\mathrm{n}, \mathrm{f})$ at $E_{n}=14.6 \mathrm{MeV}$ which has a much higher total prompt-neutron yield. The measured spectrum is also much stiffer than the one of 
the system ${ }^{252} \mathrm{Cf}(\mathrm{sf})$, although the total prompt-neutron yield is about the same. However, when comparing the empirical prompt-neutron spectrum, already multiplied with the reference spectrum of ${ }^{252} \mathrm{Cf}(\mathrm{sf})$, which is also listed in EXFOR, with the calculated spectrum, in particular in logarithmic scale in the right panel, there is very good agreement. That means that the ratio to ${ }^{252} \mathrm{Cf}$ and the spectrum given in EXFOR are not consistent. This kind of inconsistency is not observed for any other case.

${ }^{244} \mathbf{C m}(\mathbf{s f}): \quad$ The measured spectrum is well reproduced below $6 \mathrm{MeV}$. At higher energies, the measured spectrum has an unusual shape with a dip around $9 \mathrm{MeV}$. This dip is not found in the calculated spectrum.

${ }^{245} \mathbf{C m}\left(\mathbf{n}_{\mathrm{th}}, \mathbf{f}\right)$ : The measured spectrum is well reproduced at energies below $2.5 \mathrm{MeV}$. One value at $3 \mathrm{MeV}$ seems to be in error. At higher energies, the measured spectrum shows strong fluctuations, making a comparison difficult.

${ }^{246} \mathbf{C m}$ (sf) and ${ }^{248} \mathbf{C m}(\mathbf{s f}):$ Both measured spectra are well reproduced by the calculation up to $4 \mathrm{MeV}$. There are deviations and fluctuations in the experiment at higher energies.

\section{d. Discussion:}

1. General Observations: The most salient features of this comparison are:

1. There is a qualitatively rather good reproduction of the shape of the spectra, including the structural effects. There are some deviations in the quantitative reproduction of the structure at the threshold of second-chance fission.

2. In some cases, the exponential slope of the calculated spectrum exceeds the slope of the measured spectrum. The most important deviations are found for ${ }^{238} \mathrm{U}(\mathrm{n}, \mathrm{f}), \quad E_{n}=5$ and 6 $\mathrm{MeV},{ }^{239} \mathrm{Pu}\left(\mathrm{n}_{\mathrm{th}}, \mathrm{f}\right)$ with respect to one experiment, ${ }^{243} \mathrm{Am}(\mathrm{n}, \mathrm{f}), E_{n}=2.9 \mathrm{MeV}$, and ${ }^{243} \mathrm{Cm}\left(\mathrm{n}_{\mathrm{th}}, \mathrm{f}\right)$.

3. There are some fluctuations in the data for which the model does not provide an explanation. The most severe cases are ${ }^{241} \mathrm{Am}(\mathrm{n}, \mathrm{f}), E_{n}=2.9$ $\mathrm{MeV},{ }^{242} \mathrm{Am}\left(\mathrm{n}_{\mathrm{th}}, \mathrm{f}\right),{ }^{243} \mathrm{Am}(\mathrm{n}, \mathrm{f}), E_{n}=4.5 \mathrm{MeV}$, and ${ }^{243} \mathrm{Cm}\left(\mathrm{n}_{\mathrm{th}}, \mathrm{f}\right)$.

4. Two experiments for ${ }^{239} \mathrm{Pu}\left(\mathrm{n}_{\mathrm{th}}, \mathrm{f}\right)$ give diverging results.

5. There are some inconsistencies in different data tables from the same experiment for ${ }^{243} \mathrm{Cm}\left(\mathrm{n}_{\mathrm{th}}, \mathrm{f}\right)$. There is very good agreement of the calculated prompt-neutron spectrum with the spectrum, while there are strong deviations for the ratio with respect to ${ }^{252} \mathrm{Cf}(\mathrm{sf})$.
2. Pre-fission Neutron Emission: The prefission neutrons are registered in coincidence with fission only if the excitation energy of the residual nucleus falls above its fission barrier. This causes a pronounced structure in the prompt-fission-neutron spectrum. The structure of the calculated spectrum reproduces the structure in the measured spectra rather well in most cases. In the calculations, the structure depends on the description of pre-scission neutron emission, pre-equilibrium and statistical, as well as on the excitation-energy-dependent fission probabilities of the different nuclei. In particular, the mean energy of the structure in the calculated spectra depends on the value of the fission threshold in the GEF code. In particular for even-even fissioning nuclei, the number and the nature of levels at the fission barrier below the pairing gap are subject to strong nuclearstructure effects [159] and difficult to model with a global approach. In the experiment, the width of this structure is very sensitive to the energy spread of the incoming neutrons and the energy resolution in the measurement of the emitted neutrons. The mean energy is very sensitive to the energy definition of the incoming neutrons.

3. Inverse Cross Section: The mass- and energydependent transmission coefficients for neutron emission were parametrized by using inverse capture cross-sections according to Dostrovsky et al. 140 in a slightly modified version for fast computing, as already mentioned in Sec. III J

Since the fast-neutron spectrum in fission is composed of the contributions from many emitting fragments, the use of this global description is probably a satisfactory approximation.

e. Conclusion: The model behind the GEF code is unique in the sense that it provides practically all observables from nuclear fission without any need for specific experimental information by using a single fully consistent model description for all heavy fissioning systems. The present comparison with measured prompt-neutron spectra shows good agreement in most cases, but also some deviations, mostly in the high-energy tail of the spectrum and in the structures caused by threshold effects in pre-fission neutron emission. These structures are not exactly reproduced by the calculation, although their integral strength and their position in energy deviate only little in most cases. In particular in the fission of the lighter systems at higher energies, the model does not provide enough intensity at very low energies, mostly below $0.5 \mathrm{MeV}$, in the frame of the fissioning system. Some of this additional intensity is explained by the emission during the acceleration phase, but this contribution does not reach far enough down in energy. There seems to be a source of very low-energetic neutrons with an exponential-like spectrum in the frame of the fissioning system, which is not accounted for in the model. This problem has already been discussed in [241, 251]. A possible origin of these low-energy neutrons could be the pre-acceleration emission from fragments with very 
large transmission coefficients at low energies, which are not accounted for in the global description used in the present model.

A systematic view on the experimental data suggests that the uncertainties are underestimated in several cases. There are strange fluctuations in the measured spectra for ${ }^{241} \mathrm{Am}(\mathrm{n}, \mathrm{f}), E_{n}=2.9 \mathrm{MeV}$, for ${ }^{242} \mathrm{Am}\left(\mathrm{n}_{\mathrm{th}}, \mathrm{f}\right)$, for ${ }^{243} \mathrm{Am}(\mathrm{n}, \mathrm{f}), E_{n}=4.5 \mathrm{MeV}$, and for ${ }^{245} \mathrm{Cm}\left(\mathrm{n}_{\mathrm{th}}, \mathrm{f}\right)$. Contradictory results were obtained from different experiments for ${ }^{239} \mathrm{Pu}\left(\mathrm{n}_{\mathrm{th}}, \mathrm{f}\right)$. In the energy range up to $E_{n}=7 \mathrm{MeV}$, where at least most part of the spectrum is only fed by first-chance fission, the high-energy tail of the measured spectra becomes in general stiffer with increasing energy of the impinging neutron. This trend is weaker in the calculated spectra in some cases. But the variation of the stiffness is not continuous in the data as a function of the incoming-neutron energy. Sometimes, e.g. for ${ }^{238} \mathrm{U}(\mathrm{n}, \mathrm{f})$ at $E_{n}=7 \mathrm{MeV}$, the spectrum becomes softer again with increasing energy of the incoming neutrons. Moreover, the variations from one system to another one are not consistent with the model. After a careful analysis of this problem, the situation appears to be unclear. On the one hand, the mean temperature of the emitting fragments is expected to increase with increasing incoming-neutron energy. Thus, the trend to stiffer prompt-neutron spectra found in the experiment is qualitatively expected. On the other hand, these experiments are certainly very challenging, and some results may suffer from an incompletely suppressed background of scattered neutrons. This might be the reason for some unexpected fluctuations of the logarithmic slope of the spectra from one system to another as a function of incoming-neutron energy or total promptneutron yield. More data of high quality would certainly be helpful for a better understanding of this problem.

We would like to point out a slight inconsistency between the good agreement of the ratios of most promptneutron spectra to the spectrum of ${ }^{252} \mathrm{Cf}(\mathrm{sf})$ in this section and the deviations found in the previous section between the measured and the calculated spectrum of ${ }^{252} \mathrm{Cf}$ at energies below $5 \mathrm{MeV}$, while the spectrum of ${ }^{235} \mathrm{U}\left(\mathrm{n}_{\mathrm{th}}, \mathrm{f}\right)$ was well reproduced. This finding suggests to perform a deeper analysis of the experimental results.

One may conclude that the GEF model provides a global view on the systematic variation of the fission observables as a function of the fissioning system and its excitation energy. It reproduces the measured promptneutron spectra in general rather well. A detailed analysis reveals three types of deviations that are found for some of the systems. The description of the structure in the prompt-neutron spectrum due to the contribution of second-chance fission suffers probably from difficulties in modeling the level densities of even-even nuclei below the pairing gap by the global approach used in the code. Furthermore, there seems to be a source for the emission of neutrons with very low energies in some systems before or slightly after scission that is not sufficiently accounted for in the model. Finally, we think that there are indications that the stiffness of the measured prompt-neutron spectra is distorted in several cases by an incompletely suppressed background of scattered neutrons. Predictions for other systems where no experimental data are available are expected to be possible with rather good quality.

\section{F. Prompt-gamma Emission}

Information on prompt-gamma emission in the fission process is important for the gamma-heating problem in reactor cores and hence is linked to reactor safety. Furthermore, it is useful from a fundamental physics point of view, in order to learn about, among others, neutron/gamma emission competition, excitation-energy sharing and generation of angular momentum at scission.

\section{Average Values}

The calculation of reactor-core temperatures is a difficult problem because of the different nuclear reactions taking place in the core and the complex processes by which heat is generated, transported and evacuated. About $10 \%$ of the energy released in the core is in the form of gamma rays. When the reactor is shut down, gamma heating is the dominant energy-deposition process for all core materials and thus the problem of gamma heating is directly related to reactor safety. However, about $40 \%$ of this energy is prompt gamma emission $(<1$ $\mathrm{ns}$ ), and the available data in evaluated data-bases have the potential for improvement. Measurements have therefore been set at the top of the high-priority nuclear-data list of the NEA/OECD 252]. Yet, since all required data cannot be measured in the laboratory, reliable model calculations are required. Most important for applications is the total energy release, the number of emitted gamma rays and their energy spectrum. Knowledge on yields at highest gamma energy is particularly important, as these photons can travel over larger distances (many centimeters) from the initial reaction site.

According to the above, for application purposes, it has become customary to consider, at first place, the prompt gamma-ray multiplicity $M_{\gamma}$, the total energy $E_{\gamma, \text { tot }}$ released in the form of prompt gammas, and the average energy $\epsilon_{\gamma}$ per gamma quantum. These bulk observables, as measured in various experiments, are given in $\mathrm{Ta}$ bles XIV to XVI and compared to a set of model calculations for spontaneous fission of ${ }^{252} \mathrm{Cf}$ and thermalneutron-induced fission of ${ }^{235} \mathrm{U}$ and ${ }^{239} \mathrm{Pu}$. Firstly, it is noticed that there still exists a non negligible scattering between the experiments, namely for the gamma-ray multiplicity. This is, for a large part, related to experimental difficulties like the gamma-ray energy threshold of the measurement and the necessarily finite time window of a prompt event. These aspects are specific to each experiment and still hard to control in an accurate way. In addition, the high-energy part of the gamma spectrum (above about $6 \mathrm{MeV}$ ) is, in general, less reliable due to 
TABLE XIV. Average properties of prompt gammas from ${ }^{235} \mathrm{U}\left(\mathrm{n}_{\mathrm{th}}, \mathrm{f}\right)$.

\begin{tabular}{c|c|c|c|c}
\hline \hline Reference & $M_{\gamma}$ & $\epsilon_{\gamma}(\mathrm{MeV})$ & $E_{\gamma, \text { tot }}(\mathrm{MeV})$ & Energy range (MeV) \\
\hline Pleasonton [255] & $6.51 \pm 0.40$ & $0.99 \pm 0.07$ & $6.43 \pm 0.30$ & $0.09-10.0$ \\
Verbinski [256] & $6.70 \pm 0.30$ & $0.97 \pm 0.05$ & $6.51 \pm 0.30$ & $0.14-10.0$ \\
Oberstedt [258] & $8.91 \pm 0.11$ & $0.85 \pm 0.02$ & $6.92 \pm 0.09$ & $0.1-6.0$ \\
FIFRELIN [254] & 7.49 & 0.90 & 6.81 & $0.15-\infty$ \\
MCHF [259] & 8.05 & 0.88 & 7.06 & $0.14-\infty$ \\
FREYA [260] & 7.8 & 0.65 & 5.1 & $0.01-\infty$ \\
GEF & 6.89 & 0.95 & 7.08 & $0-\infty$ \\
\hline \hline
\end{tabular}

Note: Overview of available results for the prompt fission gamma multiplicity $M_{\gamma}$, average energy $\epsilon_{\gamma}$, and total energy $E_{\gamma, t o t}$ of the system ${ }^{235} \mathrm{U}\left(\mathrm{n}_{\mathrm{th}}, \mathrm{f}\right)$. The last column indicates the energy range of the gamma rays that have been either measured in experiment or considered in calculation. Refs. [255, 256, 258] correspond to experiment, while Refs. 254, 259, 260] give model predictions.

TABLE XV. Average properties of prompt gammas from ${ }^{239} \mathrm{Pu}\left(\mathrm{n}_{\mathrm{th}}, \mathrm{f}\right)$.

\begin{tabular}{c|c|c|c|c}
\hline \hline Reference & $M_{\gamma}$ & $\epsilon_{\gamma}(\mathrm{MeV})$ & $E_{\gamma, \text { tot }}(\mathrm{MeV})$ & Energy range $(\mathrm{MeV})$ \\
\hline Pleasonton [261] & $6.88 \pm 0.35$ & $0.98 \pm 0.07$ & $6.73 \pm 0.35$ & $0.122-6.1$ \\
Verbinski [256] & $7.24 \pm 0.70$ & $0.94 \pm 0.05$ & $6.81 \pm 0.30$ & $0.14-10.0$ \\
Chyzh [262] & 7.52 & 0.86 & 6.47 & $0.15-10.0$ \\
FIFRELIN [254] & 7.35 & 0.95 & 6.81 & $0.15-\infty$ \\
MCHF [259] & 8.62 & 0.89 & 7.67 & $0.14-\infty$ \\
GEF & 6.92 & 0.96 & 7.15 & $0-\infty$ \\
\hline \hline
\end{tabular}

Note: Refs. 256, 261, 262] correspond to experiment, while Refs. 254, 259] give model predictions. There are no uncertainties given in Ref. 262].

TABLE XVI. Average properties of prompt gammas from ${ }^{252} \mathrm{Cf}(\mathrm{sf})$.

\begin{tabular}{c|c|c|c|c}
\hline \hline Reference & $M_{\gamma}$ & $\epsilon_{\gamma}(\mathrm{MeV})$ & $E_{\gamma, \text { tot }}(\mathrm{MeV})$ & Energy range $(\mathrm{MeV})$ \\
\hline Pleasonton [255] & $8.32 \pm 0.40$ & $0.85 \pm 0.06$ & $6.84 \pm 0.30$ & $0.09-10.0$ \\
Verbinski [256] & $7.80 \pm 0.30$ & $0.88 \pm 0.04$ & $6.84 \pm 0.30$ & $0.14-6.0$ \\
Chyzh [263] & $8.14 \pm 0.4$ & $0.94 \pm 0.05$ & $7.65 \pm 0.55$ & $0.15-10.0$ \\
Billnert [264] & $8.30 \pm 0.08$ & $0.80 \pm 0.01$ & $6.64 \pm 0.08$ & $0.1-6.0$ \\
FIFRELIN [254] & 9.50 & 0.82 & 7.82 & $0.1-\infty$ \\
MCHF [259] & 10.7 & 0.85 & 9.10 & $0.14-\infty$ \\
FREYA [260] & 8.14 & - & - & $0.01-\infty$ \\
GEF & 7.24 & 0.86 & 6.83 & $0-\infty$ \\
\hline \hline
\end{tabular}

Note: Refs. 255, 256, 263, 264] correspond to experiment, while Refs. [254, 259, 260] give model predictions.

uncertain Compton-background subtraction [253]. Model calculations can be filtered according to the experimental conditions, provided that the latter are well known. A quantitative analysis of the influence of energy threshold and time window can be found in 254.

The survey of Tables XIV to XVI shows that the GEF code provides a fairly good description for all three observables. In particular, the almost identical values of the mean gamma energy for ${ }^{235} \mathrm{U}\left(\mathrm{n}_{\mathrm{th}}, \mathrm{f}\right)$ and ${ }^{239} \mathrm{Pu}\left(\mathrm{n}_{\mathrm{th}}, \mathrm{f}\right)$ as well as the clear decrease from ${ }^{239} \mathrm{Pu}\left(\mathrm{n}_{\mathrm{th}}, \mathrm{f}\right)$ to ${ }^{252} \mathrm{Cf}(\mathrm{sf})$ of Pleasonton et al. 255, 261 and Verbinski et al. 256], who cover all three systems, are well reproduced. Only the calculated multiplicity for ${ }^{252} \mathrm{Cf}(\mathrm{sf})$ falls slightly below the range covered by the experimental values. The values are close to the results obtained with other models used in the field [257].

\section{Energy Spectrum}

a. Global Shape: In order to probe the description of gamma emission with the GEF code in further detail, we now turn to the assessment of the gamma-energy spectrum. The experimental prompt-gamma spectra for thermal-neutron-induced fission of ${ }^{235} \mathrm{U}$ and ${ }^{241} \mathrm{Pu}$ and for spontaneous fission of ${ }^{252} \mathrm{Cf}$, as measured in most recent works [258, 264, 265], are shown in Figs. 115. 117 , respectively. In some cases, the available experi- 
mental data extend to $6 \mathrm{MeV}$, only, due to the uncertainty in background subtraction. The calculated spectra have been folded with the experimental energy resolution. Doppler broadening, which is generally less important, is neglected.

Detailed experiments with very high counting statistics and large-volume, high-granularity detectors, e.g. with the Darmstadt-Heidelberg Crystal ball, have been performed for spontaneous fission of ${ }^{252} \mathrm{Cf}$. These experiments cover a $\gamma$-energy range up to $80 \mathrm{MeV}$ including the whole GDR and extending to the postulated radiation from nucleus-nucleus coherent bremsstrahlung of the accelerating fission fragments 266], which is not considered in the GEF model. Fig. 118 shows an overview of these data in comparison with the result of the GEF code up to $15 \mathrm{MeV}$.

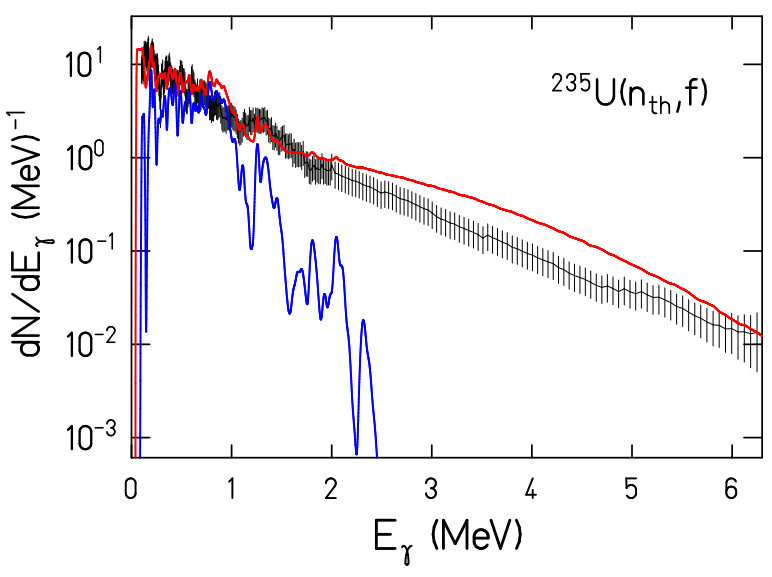

FIG. 115. (Color online) Experimental gamma-energy spectrum (black line with error bars) 264] for thermal-neutroninduced fission of ${ }^{235} \mathrm{U}$ in comparison with the GEF prediction (red line). The calculated contribution from E2 radiation is shown separately (blue line).

Overall, the comparison shows a reasonable description over the wide gamma-energy range. Note that no normalization was applied to the calculated spectrum. The relative yield of low- (below about $2 \mathrm{MeV}$ ) and highenergy gamma rays is fairly well described. Nevertheless, all calculated spectra overshoot the measured spectra between 2 and $6 \mathrm{MeV}$. This is one of the points that will be addressed in more detail below.

From the general pattern of the gamma spectrum, one can distinguish the signatures of the different contributing radiations: Statistical E1 emission dominates the high-energy part above $2 \mathrm{MeV}$, while $\mathrm{E} 2$ emission, mostly from rotational bands along the yrast line, strongly fills up the spectrum below $2 \mathrm{MeV}$. In spite of the limited experimental energy resolution, it is clear from Figs. 115 to 117 that the prompt-gamma spectrum presents structures at low-energy. These are precisely caused by the discrete rotational and vibrational transitions, as evidenced by the calculations restricted to E2 transitions

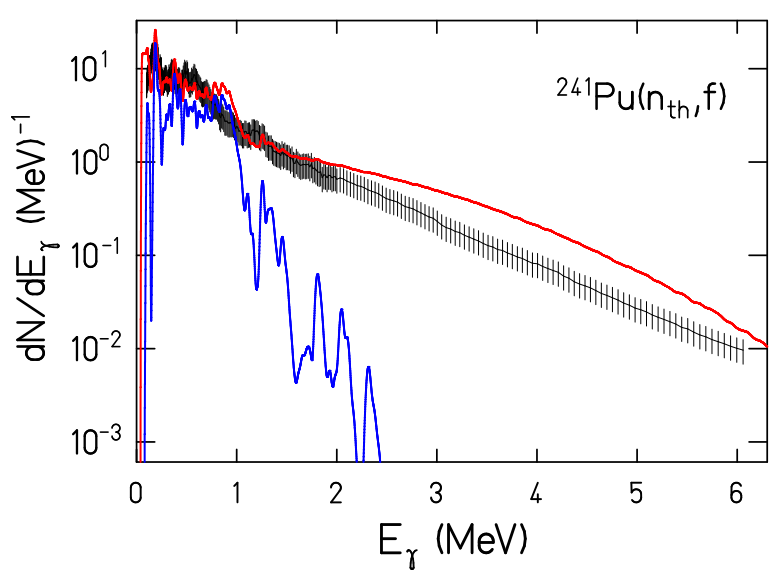

FIG. 116. (Color online) Experimental gamma-energy spectrum (black line with error bars) 265] for thermal-neutroninduced fission of ${ }^{241} \mathrm{Pu}$ in comparison with the GEF prediction (red line). The calculated contribution from E2 radiation is shown separately (blue line).

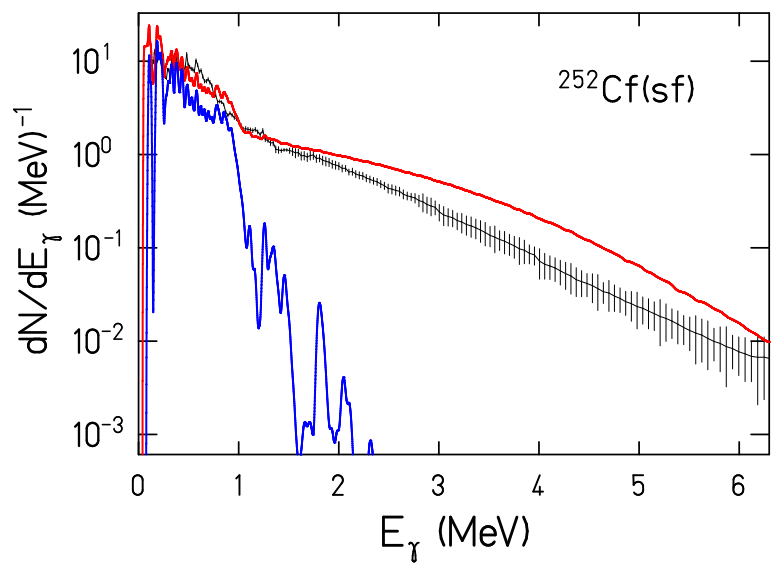

FIG. 117. (Color online) Experimental gamma-energy spectrum (black line with error bars) [258] for spontaneous fission of ${ }^{252} \mathrm{Cf}$ in comparison with the GEF prediction (red line). The calculated contribution from E2 radiation is shown separately (blue line).

(blue dashed line), and which dominate the spectrum in this region. The deviation of the calculated from the measured spectrum at very low energy is probably to a great part explained by efficiency losses of the gamma detection (the threshold does not correspond to a sharp cut: It presents a more complex uncontrolled pattern 253] and depends strongly on experimental conditions). Internal conversion (which is not modeled in GEF) does not play a major role [211]. In addition to the low-energy regime, previous inclusive measurements [269] showed an enhanced emission of medium-to-high- energy photons for specific magic fragment-mass splits. These two points have attracted much attention lately and triggered sev- 


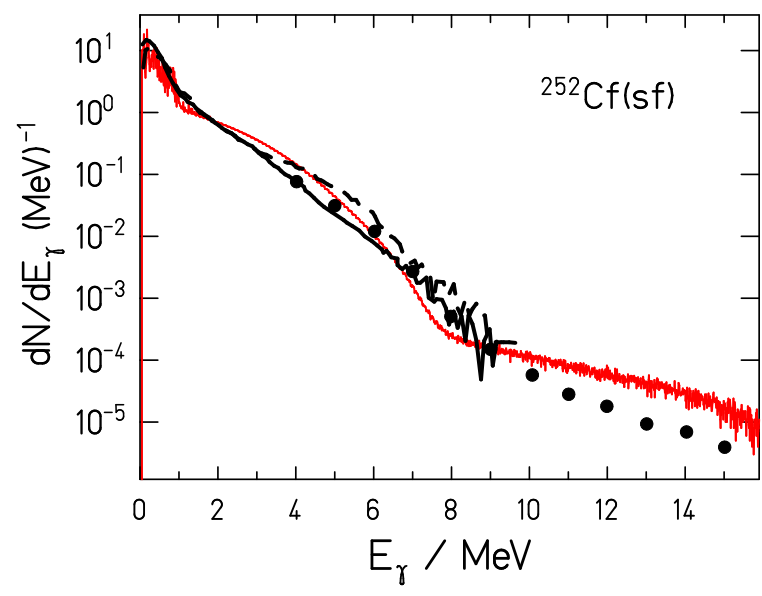

FIG. 118. Experimental prompt-gamma spectrum for ${ }^{252} \mathrm{Cf}(\mathrm{sf})$ (data points and black lines) in comparison with the result of the GEF code (red line). Black dashed line: Raw spectrum from [267], gated on the mass of the heavy fragment $126 \leq A_{H} \leq 136$. Black full line: Raw spectrum from [267], gated on $1 \overline{4} 4 \leq A_{H} \leq 154$. Full symbols: Raw data from [268]. The calculated spectrum is shown with a binsize of 10 $\mathrm{keV}$. It is not folded with the detector resolution.

eral experimental and theoretical efforts. They are addressed in some more detail in the following subsections.

The features of the spectrum at higher gamma energies in Fig. 118 are fairly well reproduced, in particular the kink near $8 \mathrm{MeV}$, approaching the peak energy of the GDR. The measured spectra have not been unfolded for the detector response. This explains most of the discrepancies between measured and calculated spectra above 9 $\mathrm{MeV}[269]$.

b. Possible Influence of the Pygmy Resonance: For all systems there appears a broad bump between 2 and 6 $\mathrm{MeV}$ in the calculated spectra that is not seen in the measured spectra. According to the calculation, the spectrum is dominated in all the range below $6 \mathrm{MeV}$ by gamma emission from states below the neutron separation energy. It seems that the first of these de-excitations rather proceed by a larger number of gammas of lower energy below $2 \mathrm{MeV}$, while transitions above $2 \mathrm{MeV}$ are suppressed. These transitions will increase the spectrum in the range below $2 \mathrm{MeV}$ by only a few percent, which would not be noticeable. Also the gamma multiplicity would increase only slightly. One possibility to explain this discrepancy in the gamma spectrum would be a more gradual decrease of the gamma strength towards lower gamma energies than expected from the tail of the giant dipole resonance that is used in the calculation. Already an energy dependence of the radiative transmission coefficient [270] $\mathcal{T} \propto E_{\gamma}^{2}$ for gamma energies below $6 \mathrm{MeV}$ would lead to much better agreement with the data. Indeed, it has been found that the gamma strength levels off and reaches an almost constant value at energies below
$3 \mathrm{MeV}$ in several tin isotopes [271]. This effect has been attributed to the influence of the pygmy resonance. The pygmy resonance is expected to be even stronger in the neutron-rich fission fragments and to dominate the lowenergy part of their gamma strength function. Thus, we tentatively attribute the absence of the calculated broad bump between 2 and $6 \mathrm{MeV}$ in the measured spectra to the influence of the pygmy resonance. Further studies of this problem are in preparation 272].

c. Low-energy Range: A more legible view on the low-energy part of the gamma spectrum is given in Figs. 119 and 120 for ${ }^{235} \mathrm{U}\left(\mathrm{n}_{\mathrm{th}}, \mathrm{f}\right)$ and ${ }^{252} \mathrm{Cf}(\mathrm{sf})$. As noted above, a distinct structure of discrete gamma peaks is observed, in spite of the limited resolution. These peaks are due to the de-excitation of the secondary fission fragments along their respective yrast bands, taking place after neutron emission and statistical (dipole) gamma emission. The GEF calculation shows a peak structure that is rather close to the experiment, however, the measurement is not fully reproduced: The comparison displayed in the figures reveals some shifts between experimental and calculated peaks. This observation is not surprising: It reflects the level of accuracy of the analytical prescription used for the modeling of the yrast line in GEF, documented in Sec. III J. Although the latter prescription has shown superior to existing analytical formalisms of this kind, as discussed in Sec. III J, deviations between experimental and calculated individual yrast energies can reach up to non negligible values, from a few tens of $\mathrm{keV}$ to a couple of $\mathrm{MeV}$ in worse cases. Such shifts are typically what can be found in Figs. 119 and 120. Additionally, these shifts can lead to different grouping of close-lying lines (see below) and further affect the comparison. That explains the mismatch in the locations of some experi-

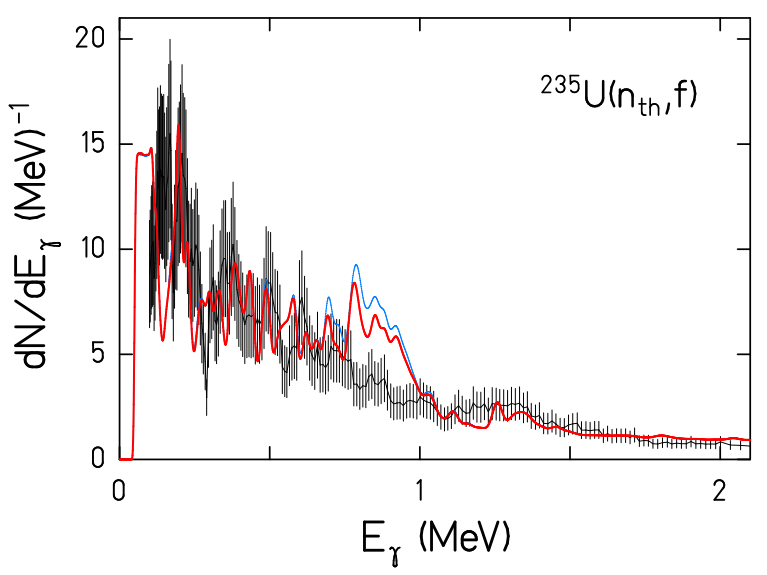

FIG. 119. (Color online) Low-energy part of the experimental gamma-energy spectrum (black line with error bars) 265] for thermal-neutron-induced fission of ${ }^{235} \mathrm{U}$ in comparison with the GEF prediction (red full line). In addition, the calculated spectrum that includes the delayed isomeric gamma transitions is shown by the light blue line. 


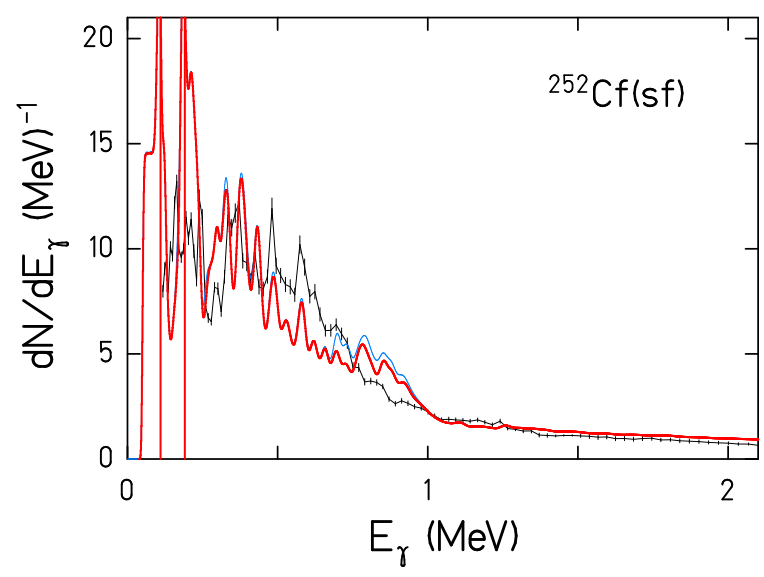

FIG. 120. (Color online) Low-energy part of the experimental gamma-energy spectrum (black line with error bars) 258] for spontaneous fission of ${ }^{252} \mathrm{Cf}$ in comparison with the $\mathrm{GEF}$ prediction (red full line). In addition, the calculated spectrum that includes the delayed isomeric gamma transitions is shown by the light blue line.

mental and calculated peaks in Fig. 119 and 120. If, instead of an analytical prescription, the full spectroscopic empirical knowledge on yrast lines is used as an input, a proper, nearly perfect, description of the low-energy part of the gamma spectrum is obtained [254, 273]. However, for many applications in nuclear technology, the promptgamma spectrum estimated by the GEF code may be suitable, considering that the calculation of prompt gammas with GEF is independent from external input of empirical fission-fragment yields, which are required for the other models. In addition, problems in matching spectroscopic information from experiment and systematics, respectively theory, are avoided, and GEF also provides predictions of similar quality in cases where fragments are formed for which no or only insufficient spectroscopic information is available.

The analysis of the low-energy structure can, in principle, give access to the identification of each contributing single fragment. The limited resolution of scintillation detectors used in the experiment discussed here (as compared to high-resolution Ge detectors) makes such a task difficult. Indeed, each peak actually contains contributions from transitions in several different fission products: These are so numerous and often have closelying transition energies, which, combined to a limited experimental resolution, then group together and form a broad peak. Nevertheless, we investigated the identity of the fragments responsible of the low-energy structure and unsurprisingly concluded to the contribution of heavy isotopes of $\mathrm{Zr}$, Mo, Ru, Pd, Sn, Sb, Te, Xe and Ba products. While the heaviest contributors do not depend much on the initial system, the relative weight of the contributors from the light fragment-mass group, of course, changes with the fissioning system. This analysis of the GEF low-energy structure of the gamma spectrum leads to the same conclusions as a similar analysis 254 performed with the FIFRELIN code, which uses empirical yrast levels. Our analysis suggests that the broad structure seen around 1.1-1.5 MeV in Figs. 115 and 116 for ${ }^{235} \mathrm{U}\left(\mathrm{n}_{\mathrm{th}}, \mathrm{f}\right)$ and ${ }^{241} \mathrm{Pu}\left(\mathrm{n}_{\mathrm{th}}, \mathrm{f}\right)$, but nearly absent in Fig. 117 for ${ }^{252} \mathrm{Cf}(\mathrm{sf})$, mainly originates from the lightest $\mathrm{Sr}$ and $\mathrm{Zr}$ products that are more abundantly produced in ${ }^{235} \mathrm{U}\left(\mathrm{n}_{\mathrm{th}}, \mathrm{f}\right)$ and ${ }^{241} \mathrm{Pu}\left(\mathrm{n}_{\mathrm{th}}, \mathrm{f}\right)$. Additionally, we note that the amount of E2 emission is constrained by the angularmomentum distribution of the fission fragments. Hence, the analysis of the low-energy part of gamma spectra with GEF may also be used to study angular-momentum generation in fission.

d. Influence of Isomeric States: There is a local overshoot of the calculated prompt-gamma spectra between $750 \mathrm{keV}$ and $1 \mathrm{MeV}$ in Figs. 119 and 120. It is particularly pronounced in the case of ${ }^{235} \mathrm{U}\left(\mathrm{n}_{\mathrm{th}}, \mathrm{f}\right)$. The key for its origin lies in the calculated total gamma spectra that include the gamma transitions that are delayed by the life-time of one or several isomers in the de-excitation gamma cascade. Note that this is a schematic calculation that assumes that the de-excitation of the primary fragments only proceeds by neutron evaporation and gamma decay. Beta decay is "switched off", and, thus, also the emission of delayed neutrons and gamma radiation after beta decay is not included. The overshoot is appreciably larger in the total spectra if compared to the calculated prompt spectra. This means that many of the gamma lines in the structure between $750 \mathrm{keV}$ and $1 \mathrm{MeV}$ of the total spectrum consist of delayed gammas, which are not contained in the measured prompt-gamma spectrum. We interpret a sizable part of the remaining overshoot of the calculated prompt-gamma spectrum by the presence of further isomeric states that are not included in the table of nuclear decay properties of JEFF 3.1.1, which are used in the GEF model. Most of those are probably not known. The concentration of isomeric transitions in this energy range may be explained by the known fact that isomers exist especially in the vicinity of doublymagic nuclei, where the gamma energies of the collective transitions at the yrast line are particularly high. Such nuclei are found in the left wings of the heavy and the light fragment groups, close to ${ }^{132} \mathrm{Sn}$ and ${ }^{78} \mathrm{Ni}$, respectively. Nuclei in both regions are, in relative, more strongly produced in fission of uranium and plutonium than in fission of californium. This explains the larger overshoot for ${ }^{235} \mathrm{U}\left(\mathrm{n}_{\mathrm{th}}, \mathrm{f}\right)$ and ${ }^{241} \mathrm{Pu}\left(\mathrm{n}_{\mathrm{th}}, \mathrm{f}\right)$ (not shown) than for ${ }^{252} \mathrm{Cf}(\mathrm{sf})$.

The presence of unknown isomers is also related to the observation of a saw-tooth shape of the mass-dependent prompt-gamma multiplicity that is discussed further below.

e. High-energy Gamma Bump: An appealing result was obtained by the Crystal-Ball collaboration [267, 269, 274] at Heidelberg for spontaneous fission of ${ }^{252} \mathrm{Cf}$ as well as several heavy-ion-induced fission reactions. A high-energy gamma-ray component, between about 3 to 
$8 \mathrm{MeV}$ and hereafter called gamma bump, was observed for specific fragment-mass partitions. This bump was ascribed to shell-related properties along the decay of the secondary neutron-rich fission products northwest of the doubly magic ${ }^{132} \mathrm{Sn}$. This interpretation is addressed with the GEF code here below.

The experimental gamma-ray spectrum as gated by different fragment-mass partitions is reported in Fig. 121 for ${ }^{252} \mathrm{Cf}(\mathrm{sf})$. For each gate, the spectrum obtained in the mass range (106-108) is shown for reference. The figure clearly reveals an excess of gamma-ray yield between about 3-8 MeV for partitions with the fragment masses between about 118 and 138. Further analysis showed that this excess originates from the heavy partner with mass around 132 of the corresponding fragment pairs. Since this gamma bump, restricted to a specific mass split, was evidenced in fission induced by different entrance channels [269, 274], the enhancement is attributed to properties of the equilibrated fission fragments rather than to effects connected with the fission process itself. The statistical-model analysis performed by the Crystal-Ball group suggested that the enhancement in high-energy gamma yield is due to the strongly reduced level densities in the vicinity of the shell closures at $Z=50$ and $N=82$.

The statistical-model calculations performed with the CASCADE code in Refs. [267, 269, 274, 275] succeeded in reproducing the gamma bump by using a prescription 216] of the nuclear level density which was deduced from measured prompt-neutron spectra as a function of fragment mass. However, these calculations are not satisfactory, because the energy-independent leveldensity parameter of Ref. 216] does not consider the well established washing out of shell effects with increasing excitation energy [276]. The level-density prescription implemented in GEF follows the widely used formulae of Ref. [64] in the constant-temperature regime and of Ref. [65] in the Fermi-gas regime (see Sec. III B). As shown in Fig. 122, also with this calculation, the gamma bump is well reproduced. In both formulae, the nuclear temperature depends on the shell-correction energies: An increased binding leads to an increase of the nuclear temperature. However, in contrast to the parametrisation of Ref. 192], this increase vanishes with increasing excitation energy. Thus, we confirm that the gamma bump results from the increase of the nuclear temperature by the shell effect that enhances the energies of the statistical gamma rays in near-closed-shell nuclei. Fig. 122 also shows another interesting feature: In parallel to the high-energy gamma bump there appears also a peak slightly below $1 \mathrm{MeV}$ that is caused by high-energetic non-statistical gamma transitions in near-magic nuclei. Also the experimental spectra (Fig. 121) show a broader low-energy peak in this energy range for the same mass windows. This is probably the same feature, appearing less clearly due to the limited resolution of the NaI crystals.

We note that the origin of the gamma bump was re-

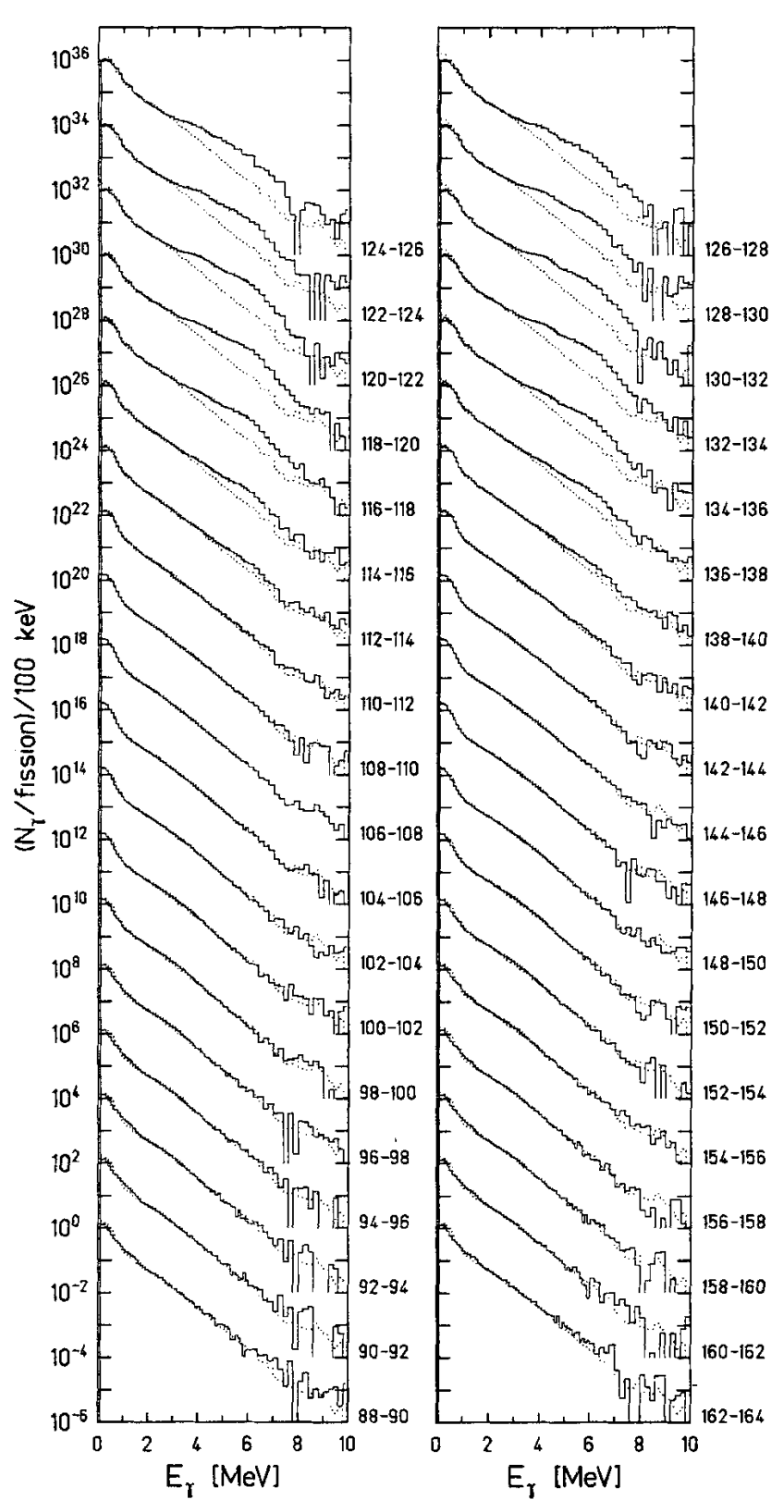

FIG. 121. (Color online) Experimental normalized gammaenergy spectra for different fragment mass splits scaled up by factors of 1, 100, 10000 , etc, for ${ }^{252} \mathrm{Cf}(\mathrm{sf})$. The dotted line shows the spectrum observed for the mass range (106-108). Extracted from Ref. [269].

cently addressed also with the FIFRELIN code 254. The results show a dominant contribution of the high-lying excited states in $\mathrm{Sb}$ isotopes close to $N=82$. However, the corresponding gamma-rays are found to be placed at the low-energy side of the experimental gamma bump, below $4 \mathrm{MeV}$. Although Regnier did not discuss it, the shape of the FIFRELIN spectrum gated on the neutron-rich $\mathrm{Sb}$ isotopes exhibits also an excess of gamma-ray yield above 


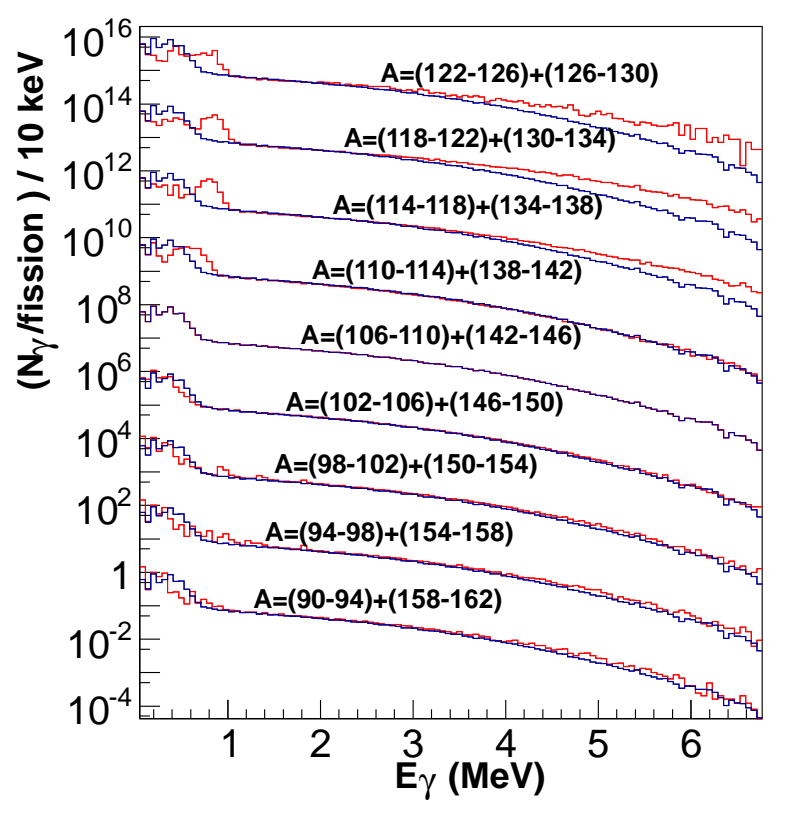

FIG. 122. (Color online) Calculated normalized gammaenergy spectra for different fragment mass splits (red lines) as obtained with the GEF code, and scaled up by suited factors for clarity for ${ }^{252} \mathrm{Cf}(\mathrm{sf})$. The blue line shows the spectrum for the mass range (106-110), or, equivalently, (142-146).

$5 \mathrm{MeV}$, where the spectrum is continuous. It may be that this observation offers a similar interpretation than the one extracted with the GEF code, namely that the gamma bump exists of statistical gammas rather than of gammas from discrete transitions along the yrast line.

\section{Saw-tooth Gamma Multiplicity}

Several measurements have been performed between the mid 60 s and early 90 s (see e.g. Refs. 255, 261, 277280]) to investigate the possible dependence of the gamma multiplicity on mass partition, and even as a function of single fragment mass. Ingenious methods (see e.g. Refs. [277, 278, 281, 282]) were developed to attribute a given detected photon to a specific fragment of the pair. These studies suggested a saw-tooth shape for $M_{\gamma}$ as a function of fragment mass, reminiscent of the sawtooth neutron-multiplicity pattern (see Sec. IX D), for several spontaneous fission and neutron-induced fission reactions. This observation is commonly linked to the fission-fragment deformation at scission (which in lowenergy fission is strongly connected to the structure of the nascent fragments) [203]. Fig. 123 shows the gamma multiplicity as a function of fission-fragment mass as extracted from measurements, as well as the prediction by the GEF code. Two observations attract attention. Firstly, the scattering of the experimental data points is noteworthy; different measurements suggest a dip in the fragment mass region $\approx 125$ - 135 with strongly varying magnitude. Secondly, while the GEF code reasonably describes the average multiplicity in the light and heavy fragment group, as well as the increasing trend with increasing mass, it is clear that it is not able to reproduce the pronounced aforementioned dip. Calculations by other authors, including the FIFRELIN 254 and Los Alamos MCHF 259, 273] models, did not converge neither: They can more or less reasonably reproduce the experimental saw-tooth pattern, but only with specific adjustment of model parameters, and at the price of losing consistency with the neutron data. Results obtained with the default parameters of the Los Alamos MCHF code 259, 273 that are consistent with the neutron data, yield results which, in magnitude and shape, are rather close to the GEF predictions (see Fig. 123).

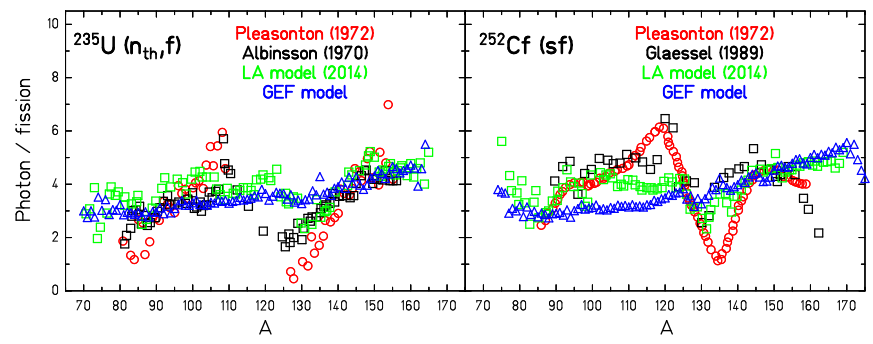

FIG. 123. (Color online) Gamma multiplicity as a function of fission-fragment mass for ${ }^{235} \mathrm{U}\left(\mathrm{n}_{\mathrm{th}}, \mathrm{f}\right)$ (left) and ${ }^{252} \mathrm{Cf}(\mathrm{sf})$ (right). Data extracted from Refs. 255, 261, 279, 280] (red and black symbols) are compared to Los Alamos MCHF 259, 273] (green squares) and GEF (blue triangles) calculations.

At first, it is not obvious that the saw-tooth behavior of the prompt-neutron yield and the saw-tooth behavior of the prompt-gamma multiplicity have the same origin, because the prompt gammas essentially are emitted from states below the respective neutron separation energy that does not depend on the initial excitation energy. Only in the rare cases when the initial fragment excitation energies falls below the neutron separation energy, the gamma multiplicity is sensitive to the initial excitation energy. Other possible effects that could be inserted in the calculation for reproducing the observed saw-tooth behavior would be a variation of the fragment angularmomentum distribution that leads to a corresponding variation of the number of non-statistical gammas or a strong decrease of the level-density parameter in magic nuclei that leads to a harder gamma spectrum and a reduced gamma multiplicity.

A very careful and well founded analysis about the existence of a saw-tooth-shaped mass-dependent gamma multiplicity has been presented in Ref. [280]. The authors conclude that the saw-tooth shape is essentially caused by deficiencies in the calibration of the fission detectors in the early experiments, because sufficiently refined procedures were not available at that time. Still, one should 
also consider another feature that reduces the multiplicity of prompt gammas in specific regions of the mass distribution: The fragments in the region of the dip have, for many of them, more or less long-lived isomeric states. Hence, depending on the size of the experimental time window used to select prompt events, existing isomeric states with lifetimes more than typically $1 \mu$ s in neutronrich $\mathrm{Sn}$ isotopes and neighboring nuclei, and transitions from these states are of course not detected. That leads to a decrease of the gamma multiplicity, and an unrealistically low spin may be deduced from the low $M_{\gamma}$ [283]. For ${ }^{235} \mathrm{U}\left(\mathrm{n}_{\mathrm{th}}, \mathrm{f}\right)$, the dip occurs in a region where fragment yields are particularly small, what does not facilitate the extraction of statistically significant and robust mass-gated $M_{\gamma}$ values. The critical influence of the presence of isomers, combined with the experimental conditions, was confirmed in recent MCHF calculations [273]: A nearly monotonic pattern for $M_{\gamma}$ as function of fragment mass is obtained when timing conditions are leveled off. Finally, we note that there are certainly many isomers that are still unknown and thus absent from available data bases. This argument was already mentioned in Sec. IX F $2 \mathrm{~d}$

The omission of these waiting points in the model calculation yields a predicted gamma multiplicity, which is larger than the measured one, and thus does not exhibit a dip. It should also be mentioned that, since the measurements of the early $90 \mathrm{~s}$, no recent experiment on this issue exists. A few measurements were performed at higher energies [189], and no very strong dip was observed. Yet, these data may not be best suited due to washing out of shell effects at these energies. Data relying on the prompt-gamma-spectroscopy method 283] at an excitation energy around $20 \mathrm{MeV}$ could not firmly conclude neither, due to the experimental bias caused by low yields and the presence of isomers. Finally, we mention the low-energy fission data of Naik et al. [203], based on the measurement of isomeric yield ratios: A clear signature of a pronounced saw-tooth was not confirmed neither, due to the scattering of the few data points and the model dependence of any extracted $M_{\gamma}$ value.

According to the above, including the un-ability of existing model calculations to describe the strong saw-tooth pattern of $M_{\gamma}$, on one side, and the likely influence of experimental bias, the question of the very existence of a pronounced saw-tooth gamma multiplicity shape is raised in the present work. A similar conclusion was suggested in Ref. [260]. All together calls for new measurements on this aspect. We are convinced that such data are of high priority as they strongly impact upon our understanding of the correlation between neutron and gamma emission, energy sharing and angular-momentum generation at scission.

\section{G. Fragment Kinetic Energies}

Another main fission observable is the kinetic energy of the fragments. The global shape of the kinetic energy as a function of fragment mass is easily reproducible: the kinetic energy can be estimated by the Coulomb repulsion between the deformed fragments. Describing the kinetic energy with high precision is however difficult.

The kinetic energies of pre- and post-neutron fragments are usually measured by the $2 v$ technique and the $2 E$ technique, respectively. The $2 E$-technique is very often used to extract the pre-neutron energies, however, a correction on mass-dependent prompt-neutron yields $\nu(A)$ must be applied. This $\nu(A)$ correction is often based on the Wahl evaluation e.g. [290]. The kinetic-energy data are usually of great quality, and the precision on the mean values is supposed to be around $0.5 \mathrm{MeV}$. The energy resolution of the detectors, however, is limited, leading to a mass resolution between 2 and 3 units [290]. The kinetic energy is also measured at the Lohengrin spectrograph, where only post-neutron fragments are available.
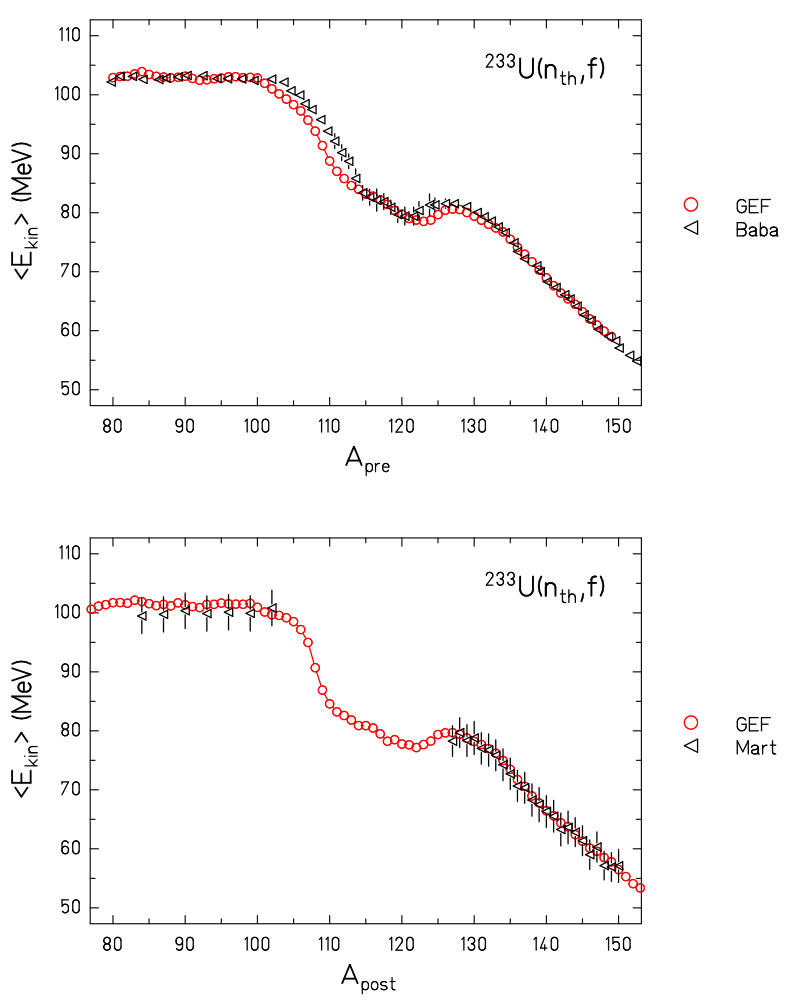

FIG. 124. (Color online) The measured mean kinetic energy before evaporation (upper part) and after evaporation (lower part) of prompt neutrons as a function of the mass of the fragment for ${ }^{233} \mathrm{U}\left(\mathrm{n}_{\mathrm{th}}, \mathrm{f}\right)$ [290, 291] is compared with the result of the GEF model.

Figs. 124 to 127 show the mean kinetic energy of the fragments for different fissioning nuclei. The agreement between the GEF predictions and the experimental data 
TABLE XVII. Mean TKE in MeV before prompt-neutron emission for well known systems.

\begin{tabular}{c|c|c|c|c}
\hline \hline Nucleus & ${ }^{233} \mathrm{U}\left(\mathrm{n}_{\mathrm{th}}, \mathrm{f}\right)$ & ${ }^{235} \mathrm{U}\left(\mathrm{n}_{\mathrm{th}}, \mathrm{f}\right)$ & ${ }^{239} \mathrm{Pu}\left(\mathrm{n}_{\mathrm{th}}, \mathrm{f}\right)$ & ${ }^{252} \mathrm{Cf}(\mathrm{sf})$ \\
\hline Recommended value & $170.1 \pm 0.5$ & $170.5 \pm 0.5$ & $177.9 \pm 0.5$ & $184.1 \pm 1.3$ \\
GEF & 172.32 & 172.04 & 178.85 & 188.14 \\
\hline \hline
\end{tabular}

Note: Mean TKE in MeV before evaporation of prompt neutrons for well known systems. The recommended values are extracted from Ref. [295], page 321.

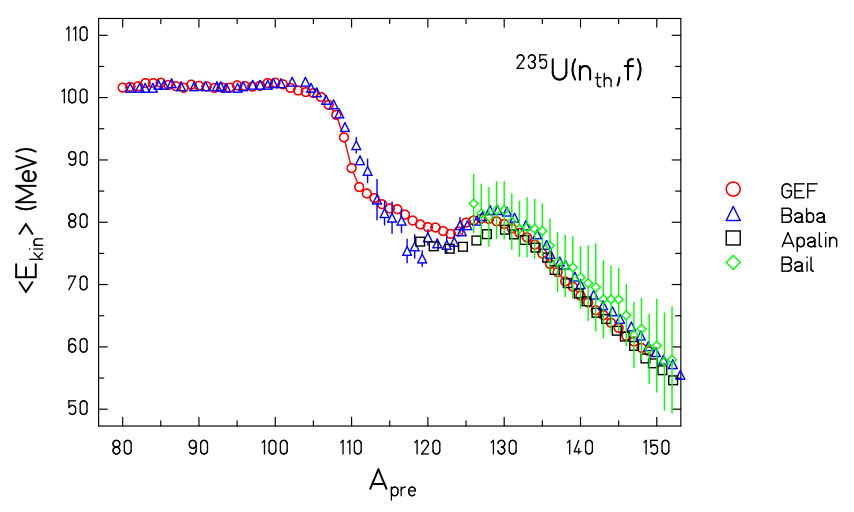

FIG. 125. (Color online) The measured mean kinetic energy before evaporation of prompt neutrons as a function of the mass of the fragment for ${ }^{235} \mathrm{U}\left(\mathrm{n}_{\mathrm{th}}, \mathrm{f}\right)$ [290, 292, 293] is compared with the result of the GEF model.

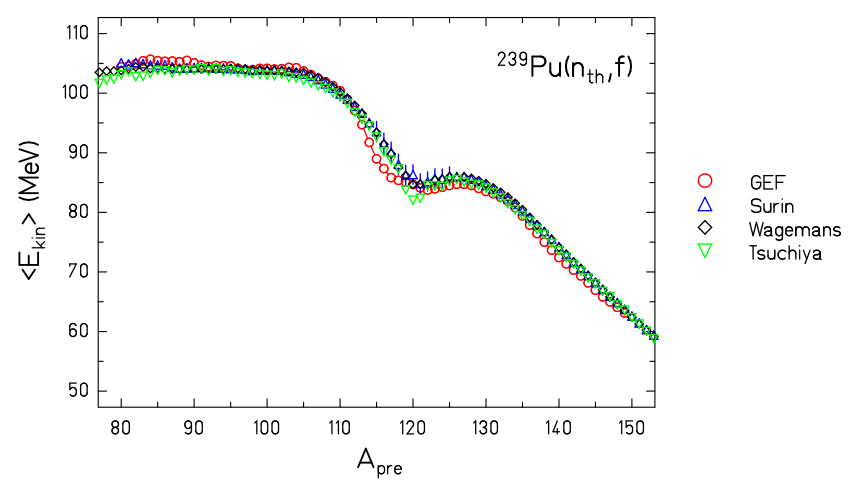

FIG. 126. (Color online) The measured mean kinetic energy before evaporation of prompt neutrons as a function of the mass of the fragment for ${ }^{239} \mathrm{Pu}\left(\mathrm{n}_{\mathrm{th}}, \mathrm{f}\right)$ [294 296] is compared with the result of the GEF model.

is very good in the thermal-neutron-induced fission of ${ }^{233} \mathrm{U},{ }^{235} \mathrm{U}$ and ${ }^{239} \mathrm{Pu}$ (see Figs. 124 to 126 ). However, the total kinetic energies of ${ }^{252} \mathrm{Cf}(\mathrm{sf})$ from the GEF model are higher by $4 \mathrm{MeV}$ than the measured data (see Fig. 127 and Table XVII).

Important deviations are also seen in the kinetic energy of neutron-induced fission of ${ }^{232} \mathrm{Th}$ (see Fig. 128) especially in the $A=120-130$ region i.e. in the border region of the SL and the S1 fission channel. This problem will be further investigated in Sec. XIB

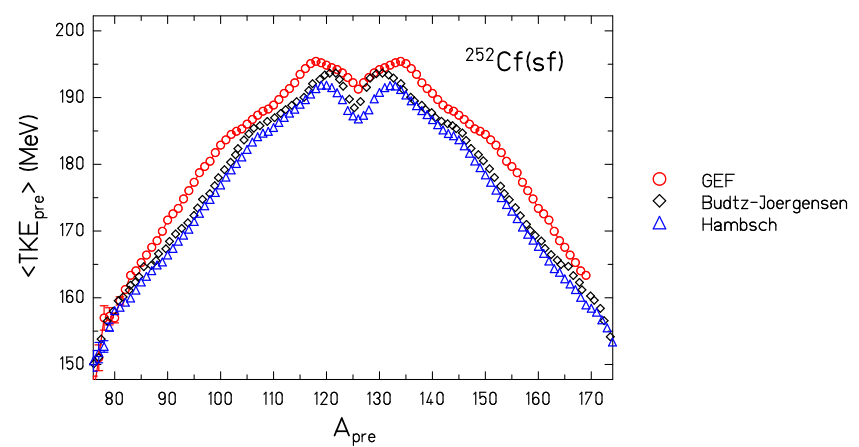

FIG. 127. (Color online) The measured mean total kinetic energy before evaporation of prompt neutrons as a function of the mass of the fragment for ${ }^{252} \mathrm{Cf}(\mathrm{sf})$ [216, 234] is compared with the result of the GEF model.

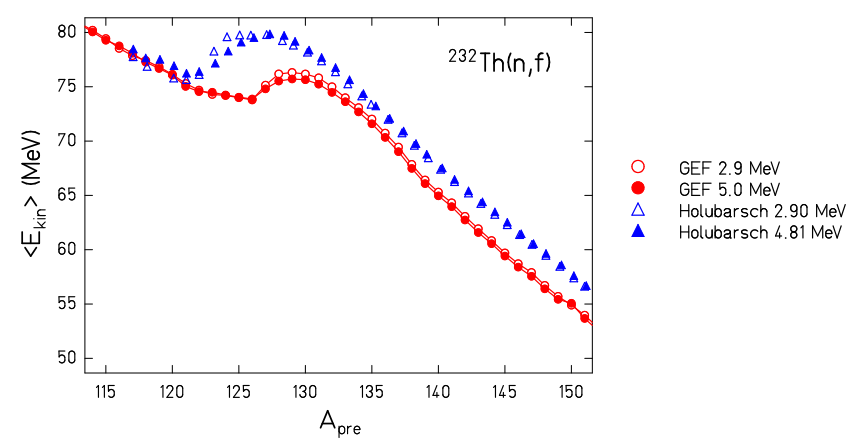

FIG. 128. (Color online) The measured mean kinetic energy before evaporation as a function of the mass of the fragment for ${ }^{232} \mathrm{Th}(\mathrm{n}, \mathrm{f})$ [297] is compared with the result of the GEF model.

Small deviations for ${ }^{232} \mathrm{Th}(\mathrm{n}, \mathrm{f})$ can also be observed in the regions of the S1 and the SL modes; they can be due to a wrong correction of prompt-neutron multiplicity $(\nu(A))$ for the experimental data when extracted from $2 E$ technique or to an underestimation of the $\operatorname{TKE}(\mathrm{S} 1)$ in the modeling of the $\mathrm{S} 1$ mode. The fission of the ${ }^{240} \mathrm{Pu}$ compound nucleus, either in neutron-induced fission or in spontaneous fission, gives some answers about the energy contribution of the S1 mode as its yield contribution is 
different (see Table XVIII).

It was observed that nearly all total-kinetic-energy distributions in neutron-induced fission have a shape close to a Gaussian with some skewness, which is well reproduced by the GEF code, see Sec. III F. An example is shown in Fig. 129 for the ${ }^{239} \mathrm{Pu}+\mathrm{n}$ reaction. For the spontaneous fission of ${ }^{238} \mathrm{Pu},{ }^{240} \mathrm{Pu}$ and ${ }^{242} \mathrm{Pu}$ a second component appears due to the large weight of the $\mathrm{S} 1$ mode. The component is also seen in the GEF calculated distribution as shown in Fig. 129. The calculated distributions are slightly shifted and systematically narrower than the measured ones. It has already been noted in [299] that the data of Milton et al. 298] should be increased by $4 \mathrm{MeV}$ to correspond to the recommended value for ${ }^{239} \mathrm{Pu}$ obtained in several measurements with the $2 v$ technique.

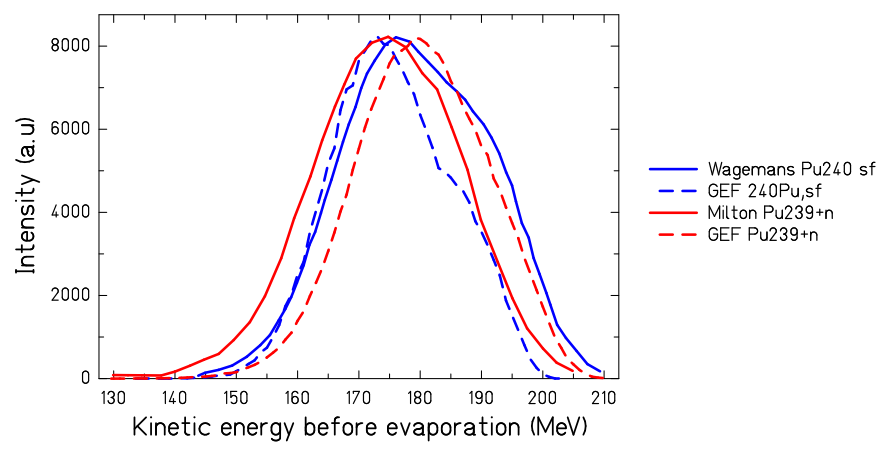

FIG. 129. (Color online) Total-kinetic-energy distribution before prompt-neutron evaporation for ${ }^{240} \mathrm{Pu}\left(\mathrm{n}_{\mathrm{th}}, \mathrm{f}\right)$ and ${ }^{240} \mathrm{Pu}(\mathrm{sf})$ [295, 298] in comparison with the result of the GEF model.

TABLE XVIII. Relative contributions of the different modes for ${ }^{239} \mathrm{Pu}\left(\mathrm{n}_{\mathrm{th}}, \mathrm{f}\right)$ and ${ }^{240} \mathrm{Pu}(\mathrm{sf})$ according to the GEF model.

\begin{tabular}{c|c|c|c}
\hline \hline & S1 & S2 & SA \\
\hline${ }^{240} \mathrm{Pu}(\mathrm{sf})$ & $16,2 \%$ & $83.2 \%$ & $0.6 \%$ \\
${ }^{239} \mathrm{Pu}+\mathrm{n}$ & $7.7 \%$ & $88.9 \%$ & $2.8 \%$ \\
\hline \hline
\end{tabular}

Though the mean TKE value is, in a lot of cases, in very good agreement, the dispersion of this value should also be studied. Fig. 130 illustrates the variance of the TKE distribution for neutron-induced reactions. The order of magnitude and the $Z$ dependence is well reproduced by the GEF model, however, the variance predicted by the GEF code is always lower than the experimental value. Because of energy conservation, a too small width in TKE also implies a too small width in the neutron multiplicity. The neutron-multiplicity distribution, as detailed in Sec. IX D, is also slightly too narrow for ${ }^{235} \mathrm{U}\left(\mathrm{n}_{\mathrm{th}}, \mathrm{f}\right)$, but the width agrees perfectly for ${ }^{239} \mathrm{Pu}\left(\mathrm{n}_{\mathrm{th}}, \mathrm{f}\right)$ and ${ }^{252} \mathrm{Cf}(\mathrm{sf})$. In view of the good agreement of the measured promptneutron multiplicity distributions with the GEF results, it may not be excluded that the influence of the experimental energy resolution is underestimated to some extent when this effect is subtracted from the width of the measured TKE distribution.

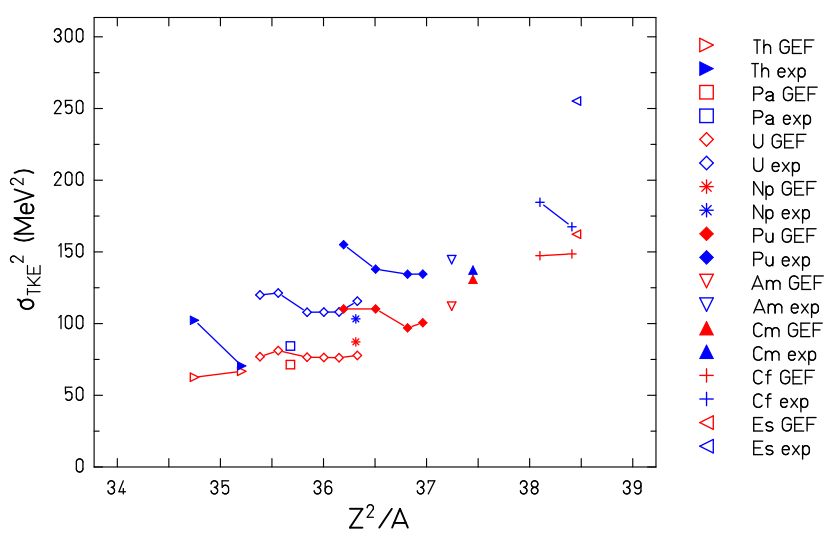

FIG. 130. (Color online) The measured variance of the TKE distribution before prompt-neutron evaporation for neutroninduced reactions [299] is compared with the result of the GEF model.

Moreover, the kinetic-energy distribution for each mass is narrower than the measured ones as shown in Fig. 131 for ${ }^{252} \mathrm{Cf}(\mathrm{sf})$.

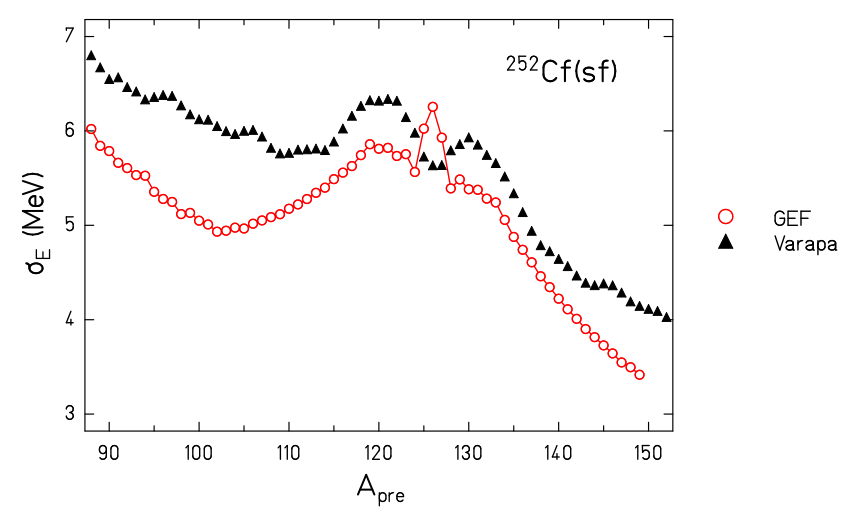

FIG. 131. (Color online) The measured mass-dependent width of the kinetic-energy distribution for ${ }^{252} \mathrm{Cf}(\mathrm{sf})$ [300] is compared with the result of the GEF model.

Fig. 132 shows the width of the kinetic energy for ${ }^{233} \mathrm{U}\left(\mathrm{n}_{\mathrm{th}}, \mathrm{f}\right)$ before and after evaporation of prompt neutrons. In agreement with the previous conclusions, the calculated width before evaporation is always narrower than the measured ones. It has to be noted that the experimental data (e.g Martin and Faust ones on Fig. 132) for the width of the kinetic-energy distribution can show some discrepancies, mainly due to the correction of the influence of the target thickness on the width of the energy 
distribution. The situation is not so clear after evaporation, where the GEF results agree with the data of Faust et al. for the light fragments. The calculated values are, however, smaller than all measured values in the heavy group.

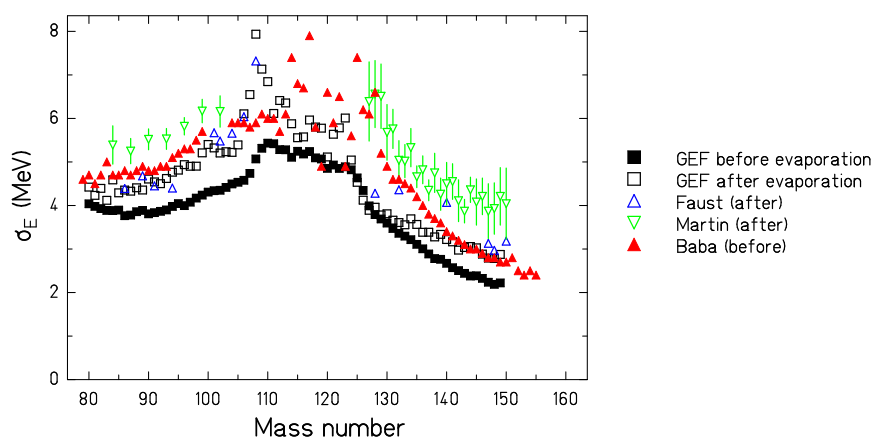

FIG. 132. (Color online) The measured width of the kineticenergy distribution for ${ }^{233} \mathrm{U}\left(\mathrm{n}_{\mathrm{th}}, \mathrm{f}\right)$ before evaporation (full symbols) and after evaporation (open symbols) 290, 291, 301] are compared with the result of the GEF model.

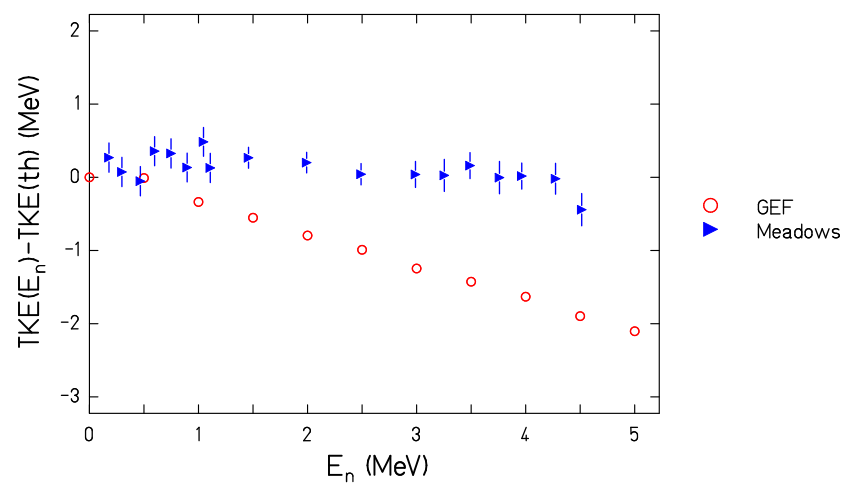

FIG. 133. (Color online) Difference between the total kinetic energy $\left(\operatorname{TKE}\left(E_{n}\right)\right)$ and the thermal value $(\operatorname{TKE}(\mathrm{th}))$ as a function of the neutron energy for ${ }^{235} \mathrm{U}(\mathrm{n}, \mathrm{f})$. Measured data [303] are compared with the result of the GEF model.

According to a lot of experiments (Ref. 299], page 366) it was observed that when increasing the excitation energy of the system the mean TKE does not change a lot. For example for ${ }^{235} \mathrm{U}(\mathrm{n}, \mathrm{f}) \operatorname{TKE}(5 \mathrm{MeV})$ - TKE(th) was observed to be around $-1 \mathrm{MeV} \pm 0.5 \mathrm{MeV}$ 107, 302, 303. which represents $0.5 \%$ of the TKE(th). This difference is of $2 \mathrm{MeV}$ for the ${ }^{239} \mathrm{Pu}(\mathrm{n}, \mathrm{f})$ reaction. However, the influence of the excitation energy of the system on the mean TKE is clearly overestimated by the GEF code as shown in Fig. 133. Due to energy conservation, the number of neutrons emitted should also evolve too much with the excitation energy of the system, by about 0.2 neutrons on $5 \mathrm{MeV}$. But this is not so much seen in Fig. 77 in
Sec. IX D. Possible explanations could be an increased mean kinetic energy of the prompt neutrons or an enhanced gamma emission. This demonstrates, how the interconnection between different fission observables can be studied by the GEF model and how this interconnection is important to reach a deeper understanding of the fission process.

\section{DATA FOR APPLICATION}

Nuclear industry strongly relies on the values of some specific fission yields. A short overview of some important features of the nuclear-reactor industry, where fission yields are important, is presented below.

\section{A. Decay Heat}

The isotopic fission yields are used in order to evaluate the decay heat. In a lot of cases, the decay data are the main problem of the decay-heat prediction, however, as shown in 304, fission yields are also of importance for the prediction.

The decay-heat calculation was performed for ${ }^{235} \mathrm{U}$ for a fission pulse at thermal energy, see Fig. 134. The GEF results as shown in the figure agree quite well with the JEFF ones. The discrepancies between the experimental data 306] and the calculated decay heat are mainly due to the decay data [307].

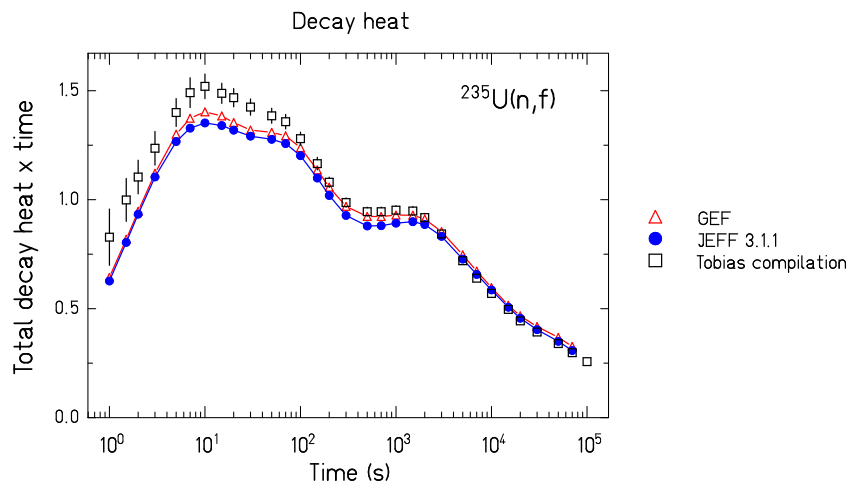

FIG. 134. (Color online) Total decay heat for a fission pulse for ${ }^{235} \mathrm{U}\left(\mathrm{n}_{\mathrm{th}}, \mathrm{f}\right)$. The calculations were performed with 305] with different fission yields.

\section{B. Delayed Neutrons}

In order to calculate the delayed fission-neutron yield $\nu_{d}$, the code implemented in [308] and the associated delayed-neutron-precursor values were used. This procedure was validated for ${ }^{235} \mathrm{U}\left(\mathrm{n}_{\mathrm{th}}, \mathrm{f}\right)$. When the JEFF 3.1.1 
fission yields are used, the calculated value of $100 \cdot \nu_{d}$ is 1.61; the recommended value is 1.62 [309].

As the number of the main delayed-neutron precursors is limited $\left({ }^{87} \mathrm{Br},{ }^{137} \mathrm{I},{ }^{88} \mathrm{Br},{ }^{138} \mathrm{I},{ }^{93} \mathrm{Rb},{ }^{89} \mathrm{Br},{ }^{94} \mathrm{Rb},{ }^{139} \mathrm{I}\right.$, $\left.{ }^{85} \mathrm{As},{ }^{98 m} \mathrm{Y},{ }^{93} \mathrm{Kr},{ }^{144} \mathrm{Cs},{ }^{140} \mathrm{I},{ }^{91} \mathrm{Br},{ }^{95} \mathrm{Rb},{ }^{96} \mathrm{Rb},{ }^{97} \mathrm{Rb}\right)$, the delayed-neutron yield allows observing some local discrepancies of the fission yields, which were found to be in good agreement with the empirical data in Sec. IX B at the first order.

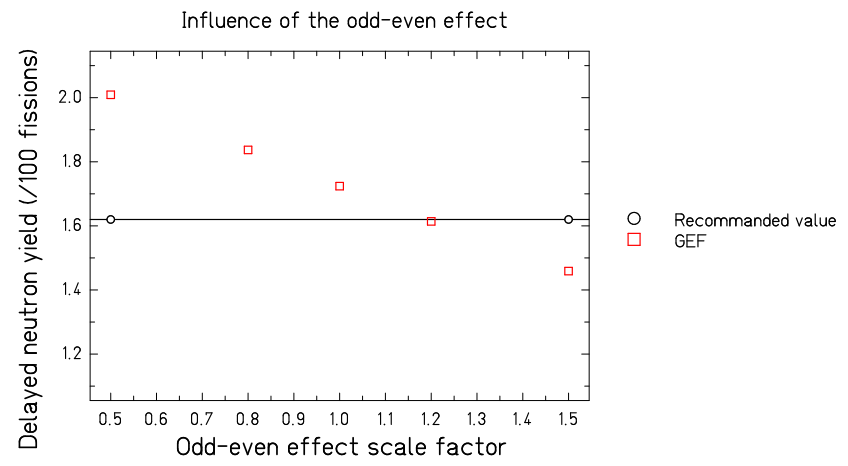

FIG. 135. (Color online) Influence of the odd-even effect on the ${ }^{235} \mathrm{U}\left(\mathrm{n}_{\mathrm{th}}, \mathrm{f}\right)$ calculated delayed-neutron (DN) yield in comparison with the recommended value 309].

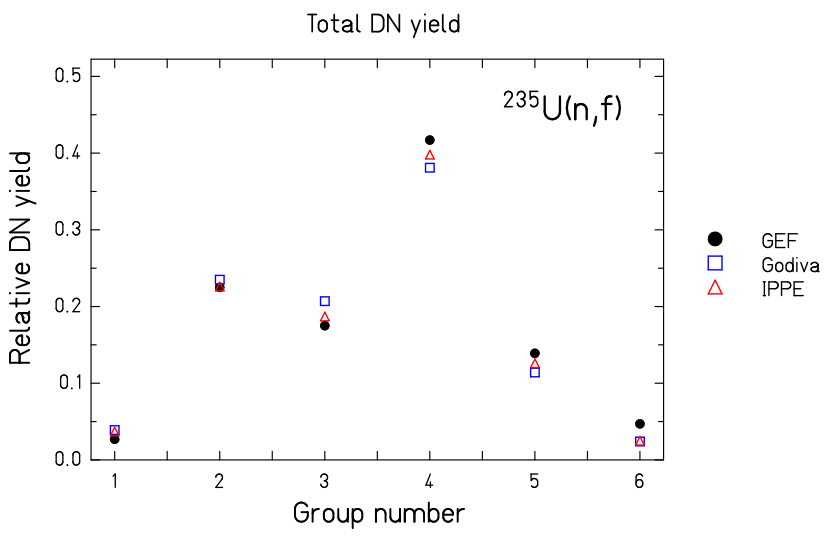

FIG. 136. (Color online) Relative delayed-neutron (DN) yield for ${ }^{235} \mathrm{U}(\mathrm{n}, \mathrm{f})$ at $E_{n}=1 \mathrm{MeV}$ as a function of the group number as defined by Keepin et al. in Annex 1. of Ref. [310]

Moreover, the delayed-neutron precursors are usually odd- $Z$ nuclei. The delayed neutrons are consequently an indirect way to observe the even-odd effect. Fig. 135 illustrates the influence of the odd-even effect on the delayedneutron yields for ${ }^{235} \mathrm{U}$. When the odd-even effect is modified by multiplying the local odd-even effect obtained by the GEF code by a scaling factor. When the scaling factor is larger (smaller) than one, the odd yields decrease (increase) and then the delayed-neutron yield decreases (increases), as observed in Fig. 135.
TABLE XIX. Delayed-neutron yields.

\begin{tabular}{c|c|c|c|c|c}
\hline \hline & ${ }^{235} \mathrm{U}$ & ${ }^{235} \mathrm{U}$ & ${ }^{238} \mathrm{U}$ & ${ }^{239} \mathrm{Pu}$ & ${ }^{239} \mathrm{Pu}$ \\
thermal & fast & fast & thermal & fast \\
\hline $\mathrm{NEA}$ & 1.62 & 1.63 & 4.65 & 0.65 & 0.651 \\
$\mathrm{GEF}$ & 1.72 & 1.64 & 4.40 & 0.71 & 0.673 \\
$(\mathrm{GEF}-\mathrm{NEA}) / \mathrm{NEA}$ & $+6.2 \%$ & $+0.6 \%$ & $-5.4 \%$ & $+9.2 \%$ & $+3.4 \%$ \\
\hline \hline
\end{tabular}

Note: Delayed-neutron yields for well-known systems. The NEA recommended values are extracted from 309]. GEF calculations were performed at $E_{n}=2 \mathrm{MeV}$ for the fast values.

The delayed fission-yield values were also compared with the recommended values for well-known fissioning systems in Table XIX. The GEF code over-estimates the delayed-fission yield slightly in most cases.

The group (as defined by Keepin et al. in Ref. 310]) repartition obtained with the GEF fission yields is also compared with the GODIVA and IPPE measurements at $1 \mathrm{MeV}$ in Fig. 136. This repartition given by GEF is in good agreement with the measured ones.

The energy dependence of the delayed-neutron yield was also studied for ${ }^{237} \mathrm{~Np},{ }^{235} \mathrm{U}$ and ${ }^{238} \mathrm{U}$, see Fig. 137 . The experimental data show a constant behavior up to $4 \mathrm{MeV}$ and then a sharp decrease. However, the GEF results show a decreasing slope whatever the energy domain. The slope is also lower than in the experimental results.

This slope should not only be associated with the oddeven effect because, when increasing the excitation energy the odd-even effect is reduced and the delayed-fissionneutron yields should increase, which is the opposite of the experimental observation. The slope is essentially due to the decrease of the peak-to-valley ratio of the fissionfragment mass yields as a function of the excitation energy. Fig. 138 shows the fission yields of the masses with at least one main delayed-neutron precursor. The fission yields of these masses decrease with the excitation energy. The constant behavior is due to the competition between the decreasing odd-even effect and the decreasing peakto-valley ratio 311 .

There are very few data above $E_{n} \approx 6 \mathrm{MeV}$, so the energy dependence when multi-chance fission is involved is difficult to benchmark. However, some data are available at $14 \pm 1 \mathrm{MeV}$. Fig. 139 shows that the experimental data are sometimes inconsistent among each other giving an increase or a decrease of the delayed neutron yields with the excitation energy. When considering that the delayed-neutron emission probability $\left(P_{n}\right)$ does not change with excitation energy and that $\nu_{d}=\Sigma\left(Y_{i} \cdot P_{n, i}\right)$, the influence of prompt-neutron emission on the fission yields $Y_{i}$ strongly suggests a decreasing tendency. The GEF results are consistent with the experimental data. 

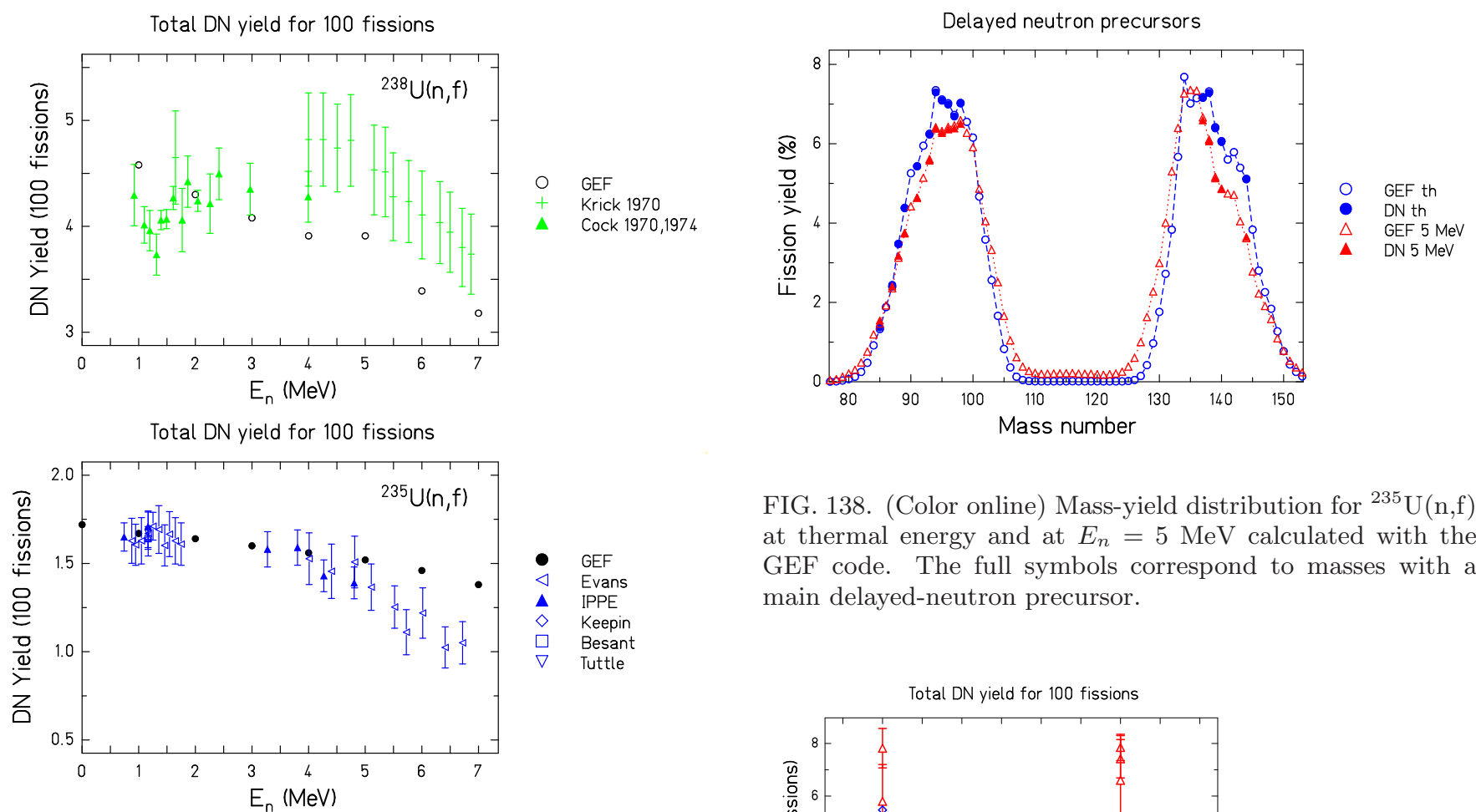

FIG. 138. (Color online) Mass-yield distribution for ${ }^{235} \mathrm{U}(\mathrm{n}, \mathrm{f})$ at thermal energy and at $E_{n}=5 \mathrm{MeV}$ calculated with the GEF code. The full symbols correspond to masses with a main delayed-neutron precursor.
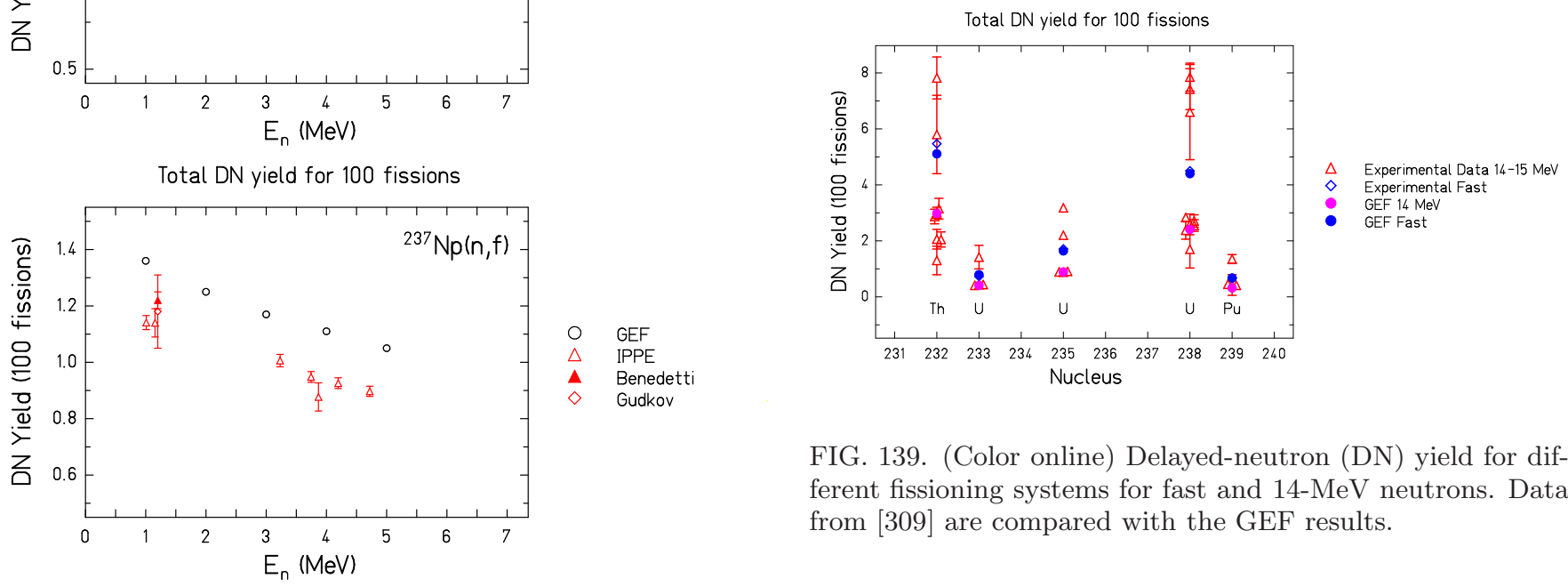

FIG. 137. (Color online) Delayed-neutron (DN) yields for ${ }^{237} \mathrm{~Np}(\mathrm{n}, \mathrm{f}),{ }^{235} \mathrm{U}(\mathrm{n}, \mathrm{f})$ and ${ }^{238} \mathrm{U}(\mathrm{n}, \mathrm{f})$, from [309] and references therein, in comparison with the GEF results.

\section{VALIDATION AND EVALUATION OF NUCLEAR DATA}

The theoretical concept of the GEF model, outlined in Sec. II, does not directly make quantitative predictions. It rather provides a rigid theoretical framework that defines a stringent link among a few key properties of the fissioning systems and practically all kind of fission observables. The quantitative predictions of the model depend on the values of a limited number of parameters, which are determined in a comprehensive way by an adjustment to a large body of various experimental data.

Considering the large number of several hundred systems that is covered by the model (not considering the

intricate variation of the fission observables with excitation energy) and the enormous complexity of the fission observables already for one system, the number of about 50 adjustable model parameters (that means far below one parameter per system) is remarkably small. These numbers elucidate that the model establishes strong relations between the different observables of one system and between the same observables of different systems. Thus, the model possesses the following fundamental virtues and constraints:

1. The model allows predicting the behavior of a specific system without any particular experimental information.

2. The model cannot be adjusted to a peculiar feature of a specific system.

The adjustment of the parameter values is difficult mainly for two reasons. First, the amount and diversity of measured fission observables is so huge that a complete 


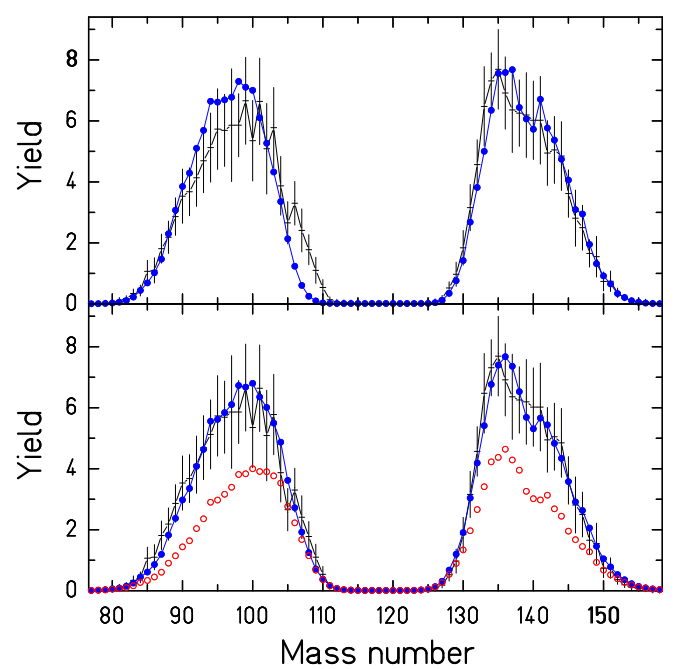

FIG. 140. (Color online) Evidence for a ${ }^{239} \mathrm{Pu}$ contaminant in a ${ }^{237} \mathrm{~Np}$ target. The fission-fragment mass distribution of the system ${ }^{237} \mathrm{~Np}\left(\mathrm{n}_{\mathrm{th}}, \mathrm{f}\right)$ from ENDF/B-VII (black crosses with error bars) in comparison with the result of the GEF code for a pure ${ }^{237} \mathrm{~Np}$ target (upper figure, blue full points) and for a composite target $\left(40 \%{ }^{237} \mathrm{~Np}\right.$ and $60 \%{ }^{239} \mathrm{Pu}$ ) (lower figure, blue full points). In addition, the contribution from the assumed ${ }^{239} \mathrm{Pu}$ contaminant is shown separately in the lower figure (open red symbols).

survey is practically impossible. Second, erroneous experimental results should be recognized and excluded from the fit procedure. This is not an easy task. However, the good agreement of the model results with the majority of the data considered in this report proves the strength of the basic concept of the GEF model and gives confidence in the reliability of the results. Thus, we propose to go a step further by using the GEF model for validating the experimental and evaluated data by verifying their consistency. In this way, the GEF model is employed for improving the quality of nuclear data. Moreover, due to its predictive power, the GEF model is used for extending the amount of nuclear data. The feasibility of this ambitious aim will be demonstrated by a few examples.

\section{A. Indications for a Target Contaminant}

The first case to be investigated is the fission-fragment mass distribution of the system ${ }^{237} \mathrm{~Np}\left(\mathrm{n}_{\mathrm{th}}, \mathrm{f}\right)$. Fig. 140 demonstrates that the evaluated spectrum can rather well be explained by a $60 \%$ (!) fission contribution from a target contaminant of ${ }^{239} \mathrm{Pu}$. This would correspond to a contamination of only $15 \mathrm{ppm}$ of ${ }^{239} \mathrm{Pu}$ in the ${ }^{237} \mathrm{~Np}$ target due to the large cross section for thermal-neutroninduced fission of ${ }^{239} \mathrm{Pu}$ that exceeds the one of ${ }^{237} \mathrm{~Np}$ by a factor of about 40000 . This demonstrates the sensitivity that can be reached in applying the GEF code for validation. An additional strong argument for the presence of a heavier target contaminant with mass number around 239 is the mean value of the mass distribution $<A>=118.03$, which would let room for the prompt emission of 1.94 neutrons for ${ }^{237} \mathrm{~Np}\left(\mathrm{n}_{\mathrm{th}}, \mathrm{f}\right)$, only. This is in contradiction to the measured value of $\bar{\nu}=2.5218$ [214].

Thus, we found two convincing arguments for the presence of an important contribution of a target impurity in the measurement underlying the evaluation of the mass distribution of the system ${ }^{237} \mathrm{~Np}\left(\mathrm{n}_{\mathrm{th}}, \mathrm{f}\right)$.

Similar considerations can be employed to investigate and eventually revise the mass distributions of other systems, for example ${ }^{254} \operatorname{Es}\left(\mathrm{n}_{\mathrm{th}}, \mathrm{f}\right)$ and ${ }^{255} \mathrm{Fm}\left(\mathrm{n}_{\mathrm{th}}, \mathrm{f}\right)$, which showed severe deviations from the GEF results (see Sec. IX B).

\section{B. An Inconsistent Mass Identification}

The next problem to be investigated is the discrepancy in the kinetic energy of neutron-induced fission of ${ }^{232} \mathrm{Th}$ between the GEF model and the experimental data found in Sec. IX G. Fig. 141 reveals that there is most probably a problem in the experimental data. According to our understanding, the kink in the kinetic-energy curve is caused by the transition from the SL to the lumped S1

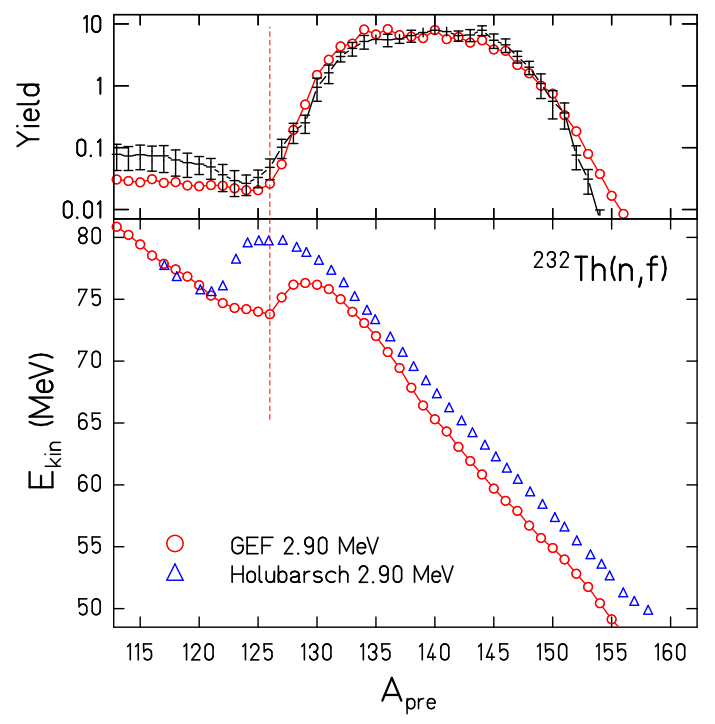

FIG. 141. (Color online) Inconsistency of mass and fragment kinetic energy for ${ }^{232} \mathrm{Th}(\mathrm{n}, \mathrm{f}), E_{n}=2.9 \mathrm{MeV}$. Fission-fragment mass distribution (upper part) and kinetic energies (lower part) of the system ${ }^{232} \mathrm{Th}(\mathrm{n}, \mathrm{f}), E_{n}=2.9 \mathrm{MeV}$ from the GEF model (red symbols) together with the evaluated mass distribution from ENDF/B-VII from fast-neutron induced fission of ${ }^{232} \mathrm{Th}$ (upper part, black crosses) and with the measured kinetic energies 297] (lower part, blue triangles). The dashed line marks the border of the asymmetric fission component according to the GEF model. 
and S2 fission-channel component with increasing mass asymmetry. This transition can also be observed by a kink in the mass distribution. In Fig. 141 this transition occurs at $A=126$ consistently in the mass distribution and in the kinetic energies from the GEF model. Also, the evaluated mass distribution for fast-neutron-induced fission is found at almost the same place. However, the kink in the measured kinetic energies is shifted by about 5 units to lower masses. This finding evokes severe doubts on the reliability of the data from Ref. [297]. The same problem appears for the measurement at $E_{n}=4.81 \mathrm{MeV}$ of the same authors.

\section{Complex Properties of Fission Channels}

It is remarkable that the GEF model is able to describe the fission-fragment distributions and their kinetic energies for all fissioning systems with a unique set of four fission channels. This is in contrast to previous work, where a complex set of channels had to be used in order to reproduce the experimental data. This becomes most evident for spontaneous fission of ${ }^{252} \mathrm{Cf}$. Table XX compares different parameterizations on the basis of the Brosa model [77] with the result of the GEF model.

TABLE XX. Yields of fission channels for the system ${ }^{252} \mathrm{Cf}(\mathrm{sf})$.

\begin{tabular}{|c|c|c|c|}
\hline Model & GEF & $\overline{\text { Brosa [312] }}$ & Brosa [313] \\
\hline $\mathrm{SL}$ & $2.6 \mathrm{E}-3 \%$ & $(3.0 \pm 0.2) \%$ & $3.1566 \%$ \\
\hline S1 & $0.54 \%$ & $(13.5 \pm 0.5) \%$ & $12.6676 \%$ \\
\hline S2 & $85.93 \%$ & $(48.2 \pm 1.1) \%$ & $46.9569 \%$ \\
\hline SA & $13.53 \%$ & $(0.3 \pm 0.1) \%$ & - \\
\hline S3 & - & $(35.0 \pm 1.2) \%$ & $0.9284 \%$ \\
\hline SX & - & - & $36.2905 \%$ \\
\hline
\end{tabular}

Note: Relative yields of the fission channels for the system ${ }^{252} \mathrm{Cf}(\mathrm{sf})$ according to different parameterizations.

${ }^{252} \mathrm{Cf}(\mathrm{sf})$ is one of the most intensively investigated systems. The mass distribution that has been determined with high precision is reproduced by the GEF model with a reduced Chi-squared of 0.62 with practically only 3 fission channels, the S1, S2 and the super-asymmetric (SA) channel. The super-long channel is so weak that it does not play any role. Also, the kinetic energies are well reproduced, except a general shift, see dedicated sections.

The reason for the smaller number and the strongly different yields of the fission channels obtained with the GEF model lies in the properties of the fission channels themselves. In the Brosa model [77], the shape of the mass distribution of all fission channels is assumed to be Gaussian. The mean total kinetic energy is parametrized by the analytical function

$$
Y(T K E)=\left(\frac{200}{T K E}\right)^{2} \cdot h \cdot \exp \left(\frac{-\left(L-l_{\max }\right)^{2}}{\left(L-l_{\min }\right) l_{d e c}}\right) .
$$

The charge-asymmetry degree of freedom enters via

$$
L=\frac{e_{0}^{2} \cdot Z_{1} \cdot Z_{2}}{T K E}
$$

For the definition of the different semi-length parameter values $l_{\max }, l_{\min }$ and $l_{d e c}$ and the population frequency $h$ see Ref. [77].

As described in Sec. VIB, in the GEF model the shape of the mass distribution of the $\mathrm{S} 2$ fission channel is given by a rectangular distribution convoluted with Gaussian distributions with different diffusenesses at the lower and the upper border. Moreover, the variation of the TKE as a function of fragment mass does not only consider the Coulomb factor $Z_{1} \cdot Z_{2}$. The TKE is also influenced by the fact that the deformation of the fragments in the different fission channels is mass dependent. This implies a different behavior of the mean TKE as a function of fragment mass.

These more complex properties of the fission channels explain the strongly different relative yields of the fission channels in the GEF model and allows describing all systems consistently with the same set of fission channels.

\section{Data Completion and Evaluation}

In many cases, the experimental data are incomplete, and it is the task of an evaluation process not only to estimate the reliability and consistency of the measured data but also to estimate the missing values with the help of systematics or theoretical models. The GEF model in combination with the dedicated optimization code MATCH [17] offers an efficient tool for this purpose.

The GEF code provides a complete set of fissionfragment yields for a specific fissioning system with uncertainties and covariances between all individual yields as determined by the model. Also many other observables with their uncertainties and covariances can be obtained, see Sec. VIII. If there are no experimental data, the GEF result may directly be used as a set of estimated values e.g. in order to extend evaluated data tables. In many cases, however, there are some experimental results available, but they are incomplete or rather uncertain. In this case, the result of the GEF code can be used for complementing the partial experimental data set in a consistent way. For this purpose, the GEF results should be adjusted in a suitable way to the experimental data.

\section{Mathematical Procedure in Two Dimensions}

In order to illustrate the solution of the problem, a schematic case in two dimensions is presented. The result of the GEF code, fission-fragment yields with their uncertainties and the covariance matrix, defines a multivariate normal distribution. This is a multi-dimensional probability-density distribution of a Gaussian-shaped 
cloud. In two dimensions, this distribution is given by

$$
\begin{array}{r}
f_{G E F}(x, y)=\frac{1}{2 \pi \sigma_{x} \sigma_{y} \sqrt{1-\rho_{x y}^{2}}} . \\
\exp \left(-\frac{1}{2\left(1-\rho_{x y}^{2}\right)}\left[\frac{\left(x-\mu_{x}\right)^{2}}{\sigma_{x}^{2}}+\frac{\left(y-\mu_{y}\right)^{2}}{\sigma_{y}^{2}}\right.\right. \\
\left.\left.-\frac{2 \rho_{x y}\left(x-\mu_{x}\right)\left(y-\mu_{y}\right)}{\sigma_{x} \sigma_{y}}\right]\right) .
\end{array}
$$

The variables are defined as follows:

$-x$ and $y$ form a two-dimensional space of possible values of two fission-fragment yields.

$-\mu_{x}$ and $\mu_{y}$ are the most probable values of the yields given by the GEF code.

$-\sigma_{x}$ and $\sigma_{y}$ are the standard deviations of the uncertainty ranges of these two yields given by GEF.

- $\rho_{x y}$ is the correlation coefficient between the variables $x$ and $y$ given by the GEF code.

$-\rho_{x y} \cdot \sigma_{x} \cdot \sigma_{y}$ is the covariance between the variables $x$ and $y$.

From this distribution, one can derive a Log-Likelihood function $L_{G E F}$ that expresses the likelihood of a set of fission-fragment yields $x$ and $y$ to be compatible with the GEF result

$$
\begin{aligned}
L_{G E F}(x, y)=\frac{1}{2\left(1-\rho_{x y}^{2}\right)} & {\left[\frac{\left(x-\mu_{x}\right)^{2}}{\sigma_{x}^{2}}+\frac{\left(y-\mu_{y}\right)^{2}}{\sigma_{y}^{2}}\right.} \\
- & \left.\frac{2 \rho_{x y}\left(x-\mu_{x}\right)\left(y-\mu_{y}\right)}{\sigma_{x} \sigma_{y}}\right] .
\end{aligned}
$$

Let us assume that there is one experimental value $x_{\text {exp }}$ available with the standard deviation of the uncertainty range $\sigma_{\text {exp }}$. The Log-Likelihood function $L_{\text {exp }}$ that expresses the likelihood of a fission-fragment yield $x$ to be compatible with the experiment is given by

$$
L_{\exp }(x)=-\frac{\left(x-x_{\exp }\right)^{2}}{2 \sigma_{x \exp }^{2}} .
$$

A set of variables $x$ and $y$ that is best compatible with both the model and the experiment may be found by constructing a combined Log-Likelihood function $L_{c}$, essentially as the sum of $L_{G E F}$ and $L_{e x p}$ and by searching the parameter values $x_{c}$ and $y_{c}$ that maximize the combined Log-Likelihood function $L_{c}$. This way, the information of the model calculation is considered in two ways: First, the absolute values deduced from the general knowledge on the physics derived from the body of available data and, secondly, the covariances that link the different yields by the inner logic of the model. However, this procedure would give more weight to the model result. Therefore, the number of yields provided by the model, in this case 2 , and the number of measured yields, in this case 1 , should be considered by enhancing $L_{\text {exp }}$ accordingly.

Thus, the proposed combined Log-Likelihood function with the appropriate weight is

$$
L_{c}=L_{G E F}+2 \cdot L_{\exp } .
$$

The exponential of the combined Log-Likelihood function (properly normalized) defines the resulting multivariate normal distribution, that is the multi-dimensional probability-density distribution of the fission yields, by which the corresponding covariance matrix is defined

It is expected that the correlations inside the model already assure that the resulting yields respect to a high degree some trivial conditions, e.g. that complementary yields are equal or the sum over the yields is normalized. In the code, the correlations of the model can be enhanced by increasing the last term in the bracket of Eq. (99), representing the model covariances. This way, the behavior of the code can be tuned.

In order to better exploit the available empirical knowledge, the dimensions of the multivariate distributions, from the model and from experiment, and the corresponding Log-Likelihood functions, Eqs. (99) and (100), can be extended to include additional quantities, for example the cumulative yields, which have often been measured rather precisely, or any other observables.

\section{Two Examples}

Figs. 142 and 143 illustrate the function of the MATCH code for the case of ${ }^{235} \mathrm{U}\left(\mathrm{n}_{\mathrm{th}}, \mathrm{f}\right)$ and ${ }^{241} \mathrm{Pu}(\mathrm{n}, \mathrm{f})$, respectively. The first case stands for a system with an almost completely measured mass distribution. Only a few yields near symmetry need to be completed. The MATCH code
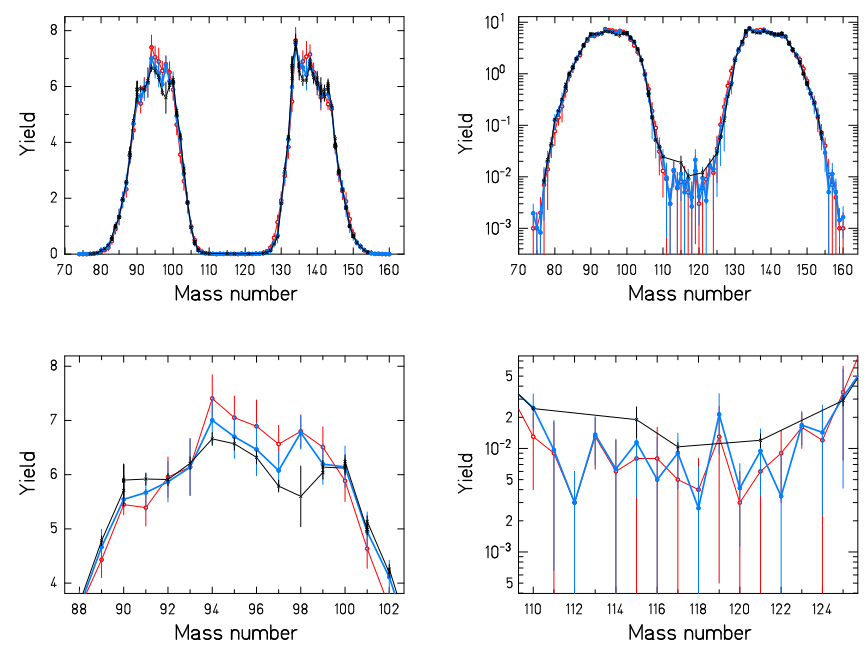

FIG. 142. (Color online) Adjustment of the fission-fragment mass yields from GEF (red symbols) to evaluated data 179. (black symbols) with the MATCH code for the system ${ }^{235} \mathrm{U}\left(\mathrm{n}_{\mathrm{th}}, \mathrm{f}\right)$. The blue symbols show the set of fission yields that maximizes the combined likelihood function of the evaluated data and the GEF result together with the covariance matrix from GEF. The upper figures show the full mass distribution in linear and logarithmic scale. The lower figures zoom on specific parts of the distribution. See text for details. 

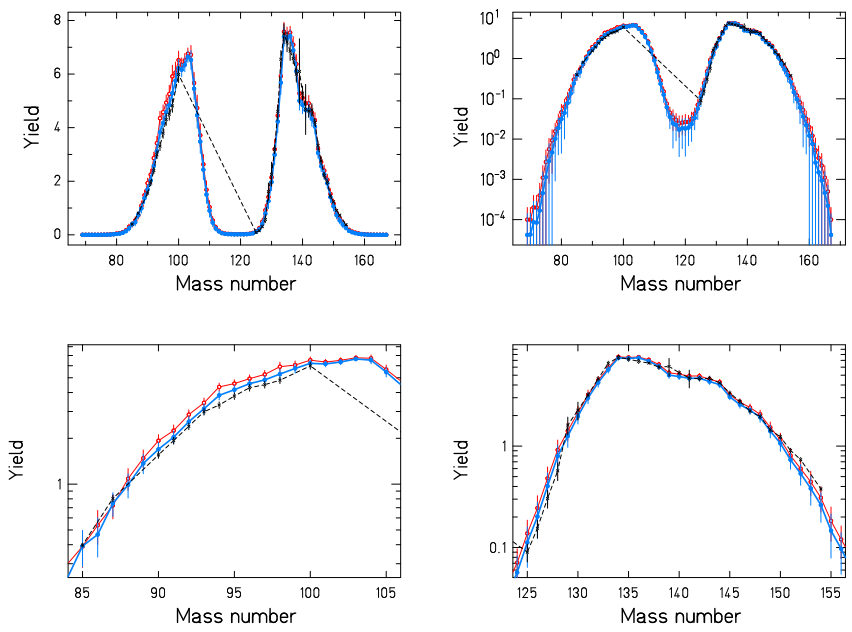

FIG. 143. (Color online) Adjustment of the fission-fragment mass yields from GEF (red symbols) to evaluated data [179] (black symbols) with the MATCH code for the system ${ }^{241} \mathrm{Pu}(\mathrm{n}, \mathrm{f}), E_{n}=2.5 \mathrm{MeV}$. The blue symbols show the set of fission yields that maximizes the combined likelihood function of the evaluated data and the GEF result together with the covariance matrix from GEF. The upper figures show the full mass distribution in linear and logarithmic scale. The lower figures zoom on specific parts of the distribution. See text for details.

was used with its default options. The data for the second system are much more incomplete. For example, there is a large gap around symmetry which is clearly seen by the straight dashed line that connects the available experimental points. In this case, the MATCH code was used with a relative weight of 100 for the experimental data and a relative weight of 10 for the covariances.

\section{CONCLUSION}

A new general approach for modeling nuclear fission on the basis of universal laws of physics and general properties of microscopic systems and mathematical objects has been derived. The most prominent features of the GEF model are the evolution of quantum-mechanical wave functions in systems with complex shape, memory effects in the dynamics of stochastic processes, the influence of the Second Law of thermodynamics on the evolution of open systems in terms of statistical mechanics, and the topological properties of a continuous function in multi-dimensional space.

It has been demonstrated that the model reproduces the measured fission barriers and the observed properties of the fission fragments, prompt neutrons and promptgamma radiation with a remarkable precision. This success reveals that the fission process possesses a high degree of inherent regularity. The suitability of the model for the evaluation of nuclear data is demonstrated.

\section{ACKNOWLEDGMENTS}

This work has been supported by the Nuclear Energy Agency of the OECD as well as by the the European Commission within the Sixth Framework Programme through EFNUDAT (project no. 036434) and within the Seventh Framework Programme through Fission-2010ERINDA (project no. 269499). We thank Mr R. Capote for drawing our attention to recent data on promptneutron spectra from fission induced by energetic neutrons. Special thanks go to Mr E. Dupont who followed this work with much interest and many helpful remarks. One of the authors (K.-H. S.) acknowledges stimulating discussions with the participants of the program on Quantitative Large Amplitude Shape Dynamics: fission and heavy ion fusion that was held at the INT of the University of Washington in Seattle, Washington, from 23 September to 15 November, 2013. We gratefully acknowledge the assistance of Mr P. Obložinský in the preparation of the manuscript and Mr. D. Hinde for a careful reading of the manuscript.
[1] K.-H. Schmidt, B. Jurado, and Ch. Amouroux, "General description of fission observables, GEF model", JEFF Report 24, NEA Data Bank of the OECD, 2014, available from http://www. oecd-nea.org/dbdata/nds_jefreports/.

[2] K.-H. Schmidt, B. Jurado, and Ch. Amouroux, "General description of fission observables, GEF model", Supplement to JEFF Report 24, NEA Data Bank of the OECD, 2014, available from http://www. oecd-nea.org/databank/docs/2014/.
[3] C. Wahl, "Nuclear-charge distribution and delayedneutron yields for thermal-neutron-induced fission of ${ }^{235} \mathrm{U},{ }^{233} \mathrm{U}$, and ${ }^{239} \mathrm{Pu}$ and for spontaneous fission of ${ }^{252}$ Cf", Atom. Data Nucl. Data Tables 39, 1 (1988).

[4] T. Asano, T. Wada, M. Ohta, T. Ichikawa, S. Yamaji, and H. Nakahara, "Dynamical calculation of multimodal nuclear fission of fermium nuclei", J. NuCL. RADIOCH. SC. 5, 1 (2004).

[5] T. Asano, T. Wada, M. Ohta, S. Yamaji, and H. Nakahara, "The dependency on the dissipation tensor of multi-model nuclear fission", J. NuCL. RAdioch. SC. 
7,7 (2006).

[6] Y. Aritomo, S. Chiba, and F. Ivanyuk, "Fission dynamics at low excitation energy", PHys. Rev. C 90, 054609 (2014).

[7] J. Randrup, P. Möller, and A. J. Sierk, "Fissionfragment mass distributions from strongly damped shape evolution", Phys. Rev. C 84, 034613 (2011).

[8] H. Goutte, J. F. Berger, P. Casoli, and D. Gogny, "Microscopic approach of fission dynamics applied to fragment kinetic energy and mass distributions in ${ }^{238} \mathrm{U}$ ", Phys. Rev. C 71, 024316 (2005).

[9] A. S. Umar, V. E. Oberacker, J. A. Maruhn, and P.G. Reinhard, "Microscopic description of nuclear fission dynamics", J. Phys. G: NuCl. PART. Phys. 37, 064037 (2010).

[10] V. V. Sargsyan, Z. Kanokov, G. G. Adamian, and N. V. Antonenko, "Quantum statistical effects in nuclear reactions, fission, and open quantum systems", PHYs. PART. NuClei 41, 175 (2010).

[11] S. Goriely, N. Chamel, and J. M. Pearson, "HartreeFock-Bogoliubov nuclear mass model with $0.50 \mathrm{MeV}$ accuracy based on standard forms of Skyrme and pairing functionals", PHYs. REV. C 88, 061302 (2013).

[12] Adam Sobiczewski and Yuri A. Litvinov, "Accuracy of theoretical descriptions of nuclear masses", PHys. REV. C 89, 024311 (2014).

[13] P.-G. Reinhard, M. Bender, W. Nazarewicz, and T. Vertse, "From finite nuclei to the nuclear liquid drop: Leptodermous expansion based on self-consistent meanfield theory", Phys. REv. C 73, 014309 (2006).

[14] S. Goriely, "The fundamental role of fission during rprocess nucleosynthesis in neutron star mergers", EUR. Phys. J. A 51, 22 (2015)

[15] https://mcnp.lanl.gov/

[16] http://www.fluka.org/fluka.php

[17] MATCH computer code, available at http://www.khs-erzhausen.de

[18] W. D. Myers and W. J. Swiatecki, "Nuclear properties according to the Thomas-Fermi model" NucL. PHYs. A 601, 141 (1996).

[19] A. V. Karpov, A. Kelic, and K.-H. Schmidt, "On the topographical properties of fission barriers", J. PHYs. G: Nucl. Part. Phys. 35, 035104 (2008).

[20] E. Wigner, "The transition state method", Trans. FARADAY SOT. 34, 29 (1938).

[21] M. Diebel, K. Albrecht, and R. W. Hasse, "Microscopic calculations of fission barriers and critical angular momenta for excited heavy nuclear systems", NuCL. PHYS. A 355, 66 (1981).

[22] J. C. Pei, W. Nazarewicz, J. A. Sheikh, and A. K. Kerman, "Fission barriers of compound superheavy nuclei", Phys. Rev. LetT. 102, 192501 (2009).

[23] For a continuous tracking of these degrees of freedom, suitable prescriptions must be defined that generalise these values that are defined for the separated fragments to the respective deformation parameters of the system on the fission path before scission. This is usually provided by the shape parameterisation.

[24] V. V. Pashkevich and A. Ya. Rusanov, "The ${ }^{226}$ Th fission valleys", NucL. Phys. A 810, 77 (2008).

[25] U. Mosel and H. W. Schmitt, "Potential energy surfaces for heavy nuclei in the two-center model", NuCL. PHYs. A 165, 73 (1971).
[26] K.-H. Schmidt, A. Kelic, and M. V. Ricciardi, "Experimental evidence for the separability of compoundnucleus and fragment properties in fission", EUROPH. LETT. 83, 32001 (2008).

[27] P. Möller, A. J. Sierk, T. Ichikawa, A. Iwamoto, R. Bengtsson, H. Uhrenholt, and S. Aberg, "Heavy-element fission barriers", Phys. Rev. C 79, 064304 (2009).

[28] G. D. Adeev and V. V. Pashkevich, "Theory of macroscopic fission dynamics", NuCl. PHYs. A 502, 405c (1989).

[29] A. Ya. Rusanov, M. G. Itkis, and V. N. Oklovich, "Features of mass distributions of hot rotating nuclei", PHYS. AT. NuCL. 60, 683 (1997).

[30] A. V. Karpov, P. N. Nadtochy, D. V. Vanin, and G. D. Adeev, "Three-dimensional Langevin calculations of fission fragment mass-energy distribution from excited compound nuclei", Phys. Rev. C 63, 054610 (2001).

[31] W. D. Myers, G. Manzouranis, and J. Randrup, "Adiabaticity criterion for charge equilibration with application to fission", Phys. LetT. B 98, 1 (1981).

[32] A. V. Karpov and G. D. Adeev, "Langevin description of charge fluctuations in fission of highly excited nuclei", Eur. Phys. J. A 14, 169 (2002).

[33] A. Al-Adili, F.-J. Hambsch, S. Pomp, and S. Oberstedt, "Impact of prompt-neutron corrections on final fissionfragment distributions", Phys. REv. C 86, 054601 (2012).

[34] D. H. Gross, "A New Thermodynamics from Nuclei to Stars", Entropy 6, 158 (2004) / Special Issue Quantum Limits to the Second Law of Thermodynamics.

[35] K.-H. Schmidt and B. Jurado, "Entropy-driven excitation-energy sorting in superfluid fission dynamics", Phys. Rev. Lett. 104, 212501 (2010).

[36] G. Boutoux, G. Belier, A. Chatillon, A. Ebran, T. Gorbinet, B. Laurent, J.-F. Martin, E. Pellereau, J. Taieb, L. Audouin, L. Tassan-Got, B. Jurado, H. Alvarez-Pol, Y. Ayyad, J. Benlliure, M. Caamano, D. Cortina-Gil, B. Fernandez-Dominguez, C. Paradela, J.-L. RodriguezSanchez, J. Vargas, E. Casarejos, A. Heinz, A. KelicHeil, N. Kurz, C. Nociforo, S. Pietri, A. Prochazka, D. Rossi, K.-H. Schmidt, H. Simon, B. Voss, H. Weick, and J. S. Winfield, "The SOFIA experiment", Phys. ProCEDIA 47, 166 (2013).

[37] CONFID computer code, available at http://www.khs-erzhausen.de.

[38] A. Kelic and K.-H. Schmidt, "Assessment of saddlepoint-mass predictions for astrophysical applications", Phys. LetT. B 634, 362 (2006).

[39] G. Royer and C. Bonilla, "Multiple-humped fission and fusion barriers of actinide and superheavy elements", J. Radioanal. Nucl. Ch. 272, 237 (2007).

[40] A. Dobrowolski, K. Pomorski, and J. Bartel, "Fission barriers in a macroscopic-microscopic model", PHYS. REv. C 75, 024613 (2007).

[41] M. Kowal, P. Jachimowicz, and A. Sobiczewski, "Fission barriers for even-even superheavy nuclei", PHYs. REv. C 82, 014303 (2010).

[42] M. Mirea and L. Tassan-Got, "Th and U fission barriers within the Woods-Saxon two center shell model", Centr. Eur. J. Phys. 9, 116 (2011).

[43] P. Jachimowicz, M. Kowal, and J. Skalski, "Secondary fission barriers in even-even actinide nuclei", PHYS. ReV. C 85, 034305 (2012). 
[44] M. Kowal and J. Skalski, "Examination of the existence of third, hyperdeformed minima in actinide nuclei", Phys. Rev. C 85, 061302(R) (2012).

[45] H. Abusara, A. V. Afanasjev, and P. Ring, "Fission barriers in covariant density functional theory: Extrapolation to superheavy nuclei", PHYs. REv. C 85, 024314 (2012).

[46] Bing-Nan Lu, En-Guang Zhao, and Shan-Gui Zhou, "Potential energy surfaces of actinide nuclei from a multidimensional constrained covariant density functional theory: Barrier heights and saddle point shapes", PHYs. REV. C 85, 011301(R) (2012).

[47] J.-P. Delaroche, M. Girod, H. Goutte, and J. Libert, "Structure properties of even-even actinides at normal and super deformed shapes analysed using the Gogny force", Nucl. Phys. A 771, 103 (2006).

[48] S. Goriely, M. Samyn, and J. M. Pearson, "Further explorations of Skyrme-Hartree-Fock-Bogoliubov mass formulas. VII. Simultaneous fits to masses and fission barriers", Phys. Rev. C 75, 064312 (2007).

[49] F. Minato and K. Hagino, "Fission barriers in the neutron-proton isospin plane for heavy neutron-rich nuclei", Phys. Rev. C 77, 044308 (2008).

[50] S. Goriely, S. Hilaire, A. J. Koning, M. Sin, and R. Capote, "Towards a prediction of fission cross sections on the basis of microscopic nuclear inputs", PHYS. REV. C 79, 024612 (2009).

[51] F. A. Ivanyuk and K. Pomorski, "Optimal shapes and fission barriers of nuclei within the liquid drop model", Phys. Rev. C 79, 054327 (2009).

[52] S. G. Nilsson, "Binding States of Individual Nucleons in Strongly Deformed Nuclei", Kgl. Danske Videnskab. Selskab, Mat.-Fys. Medd. 29, 16 (1955).

[53] W. D. Myers and W. J. Swiatecki, "Thomas-Fermi fission barriers", Phys. Rev. C 60, 014606 (1999).

[54] P. Möller, J. R. Nix, W. D. Myers, and W. J. Swiatecki, "Nuclear ground-state masses and deformations", Atom. Nucl. Data Tables 59, 185 (1995).

[55] It is the benefit of ref. [27], compared to most theoretical work, that it presents extensive tables of calculated barrier parameters of the actinides, which makes the quantitative comparison with this theory feasible.

[56] M. Baldo, U. Lombardo, E. E. Saperstein, and M. V. Zverev, "On the surface nature of the nuclear pairing", Phys. ReP. 391, 261 (2004).

[57] S. Bjoernholm and J. E. Lynn, "The double-humped fission barrier", REv. Mod. PHYs. 52, 725 (1980).

[58] R. Capote, M. Herman, P. Oblozinsky, P. G. Young, S. Goriely, T. Belgya, A. V. Ignatyuk, A. J. Koning, S. Hilaire, V. A. Plujko, M. Avrigeanu, O. Bersillon, M. Chadwick, T. Fukahori, Zhigang Ge, Yinlu Han, S. Kailas, J. Kopecky, V. M. Maslov, G. Reffo, M. Sin, E. Sh. Soukhovitskii, and P. Talou, "RIPL - Reference Input Parameter Library for calculation of nuclear reactions and nuclear data evaluations", NuCL. DATA SheETs 110, 3107 (2009).

[59] M. Dahlinger, D. Vermeulen, and K.-H. Schmidt, "Empirical saddle-point and ground-state masses as a probe of the droplet model", NuCl. Phys. A 376, 94 (1982).

[60] According to Ref. [61], the most precise and robust nuclear mass predictions are provided by the Duflo-Zuker model [62], which gives an rms deviation of $373 \mathrm{keV}$.

[61] C. Barbero, J. G. Hirsch, and A. E. Mariano, "Deformation and shell effects in nuclear mass formulas", NuCL.
PHYS. A 874, 81 (2012).

[62] A. P. Zuker, "On the microscopic derivation of a mass formula", Nucl. Phys. A 576, 65 (1994).

[63] K.-H. Schmidt and B. Jurado, "Inconsistencies in the description of pairing effects in nuclear level densities", Phys. Rev. C 86, 044322 (2012).

[64] T. von Egidy and D. Bucurescu, "Experimental energydependent nuclear spin distributions", Phys. Rev. C 80, 054310 (2009).

[65] A. V. Ignatyuk, G. N. Smirenkin, and A. S. Tishin, "Phenomenological description of the energy dependence of the level density parameter", Sov. J. NuCL. PHYs. 21, 255 (1975).

[66] A. V. Ignatyuk, "Systematics of Low-Lying Level Densities and Radiative Widths", HADrons NuCleI APPL. 3, 287 (2001).

[67] S. Bjoernholm, A. Bohr, and B. R. Mottelson, "Role of symmetry of the nuclear shape in rotational contributions to nuclear level densities, Proc. Int. Conf. on the Physics and Chemistry of Fission, Rochester 1973 (IAEA Vienna 1974) Vol. 1, p. 367.

[68] A. Gilbert and A. G. W. Cameron, "A composite nuclear-level density formula with shell corrections", CAn. J. Phys. 43, 1446 (1965).

[69] K.-H. Schmidt, S. Steinhäuser, C. Böckstiegel, A. Grewe, A. Heinz, A. R. Junghans, J. Benlliure, H.G. Clerc, M. de Jong, J. Müller, M. Pfützner, and B. Voss, "Relativistic radioactive beams: A new access to nuclear-fission studies", NuCl. Phys. A 665, 221 (2000).

[70] M. G. Itkis N. A. Kondrat'ev, S. I. Mul'gin, V. N. Okolovich, A. Ya. Rusanov, and G. N. Smirenkin, "Mass asymmetry of symmetric fission of nuclei with A approximately 200", Sov. J. Nucl. Phys. 52, 601 (1990).

[71] A. N. Andreyev, J. Elseviers, M. Huyse, P. Van Duppen, S. Antalic, A. Barzakh, N. Bree, T. E. Cocolios, V. F. Comas, J. Diriken, D. Fedorov, V. Fedosseev, S. Franchoo, J. A. Heredia, O. Ivanov, U. Koester, B. A. Marsh, K. Nishio, R. D. Page, N. Patronis, M. Seliverstov, I. Tsekhanovich, P. Van den Bergh, J. Van De Walle, M. Venhart, S. Vermote, M. Veselsky, C. Wagemans, T. Ichikawa, A. Iwamoto, P. Möller, and A. J. Sierk, "New Type of Asymmetric Fission in Proton-Rich Nuclei", Phys. Rev. Lett. 105, 252502 (2010).

[72] S. I. Mulgin, K.-H. Schmidt, A. Grewe, and S. V. Zhdanov, "Shell effects in the symmetric-modal fission of pre-actinide nuclei", Nucl. Phys. A 640, 375 (1998).

[73] J. P. Unik, J. E. Gindler, L. E. Glendenin, K. F. Flynn, A. Gorski, and R. K. Sjoblom, "Fragment mass and kinetic energy distributions for fissioning systems ranging from mass 230 to 256", Proc. Symp. Phys. Chem. Fission, Rochester 1973, IAEA Vienna (1974), vol. 2, p. 19.

[74] B. D. Wilkins, E. P. Steinberg, and R. R. Chasman, "Scission-point model of nuclear fission based on deformed-shell effects", PHys. REv. C 14, 1832 (1976).

[75] http://www. cenbg.in2p3.fr/GEF, http://www.khs-erzhausen.de.

[76] I. Ragnarsson and R. K. Sheline, "Systematics of nuclear deformations", PHYs. SCR. 29, 385 (1984).

[77] U. Brosa, S. Grossmann, and A. Müller, "Nuclear scission", Phys. Rep. 197, 167 (1990).

[78] S. I. Mulgin, V. N. Okolovich, and S. V. Zhdanov, "Observation of new channel in the proton-induced low- 
energy fission of nuclei from ${ }^{233} \mathrm{~Pa}$ to ${ }^{245} \mathrm{Bk}$ ", PHYs. LETT. B 462 29, (1999).

[79] M. G. Itkis, V. N. Okolovich, A. Ya, Rusanov, and G. N. Smirenkin, "Asymmetric fission of the pre-actinide nuclei". Z. Phys. A 320, 433 (1985).

[80] A. A. Naqvi, F. Käppeler, F. Dickmann, and R. Müller, "Fission fragment properties in fast-neutron-induced fission of ${ }^{237}$ Np", Phys. Rev. C 34, 218 (1986).

[81] W. Lang, H.-G. Clerc, H. Wohlfarth, H. Schrader, and K.-H. Schmidt, "Nuclear charge and mass yields for ${ }^{235} \mathrm{U}\left(n_{t h}, f\right)$ as a function of the kinetic energy of the fission products", Nucl. Phys. A 345, 34 (1980).

[82] P. Fong, "Statistical Theory of Nuclear Fission", (Gordon and Breach, New York, 1969).

[83] G. A. Pik-Pichak and V. M. Strutinsky, "Physics of the fission of the atomic nuclei", eds. N. A. Perfilov and V. P. Eismont, (Moscow, Gosatmomizdat, 1962) p. 12.

[84] A. V. Ignatyuk, "Statistical description of the yields of fission products", YAD. Fiz. 9, 357 (1969).

[85] Yu. Ts. Oganessian and Yu. A. Lazarev, "Heavy ions and nuclear fission", in Treatise on Heavy Ion Science, Vol. 4, ed. D. A. Bromley, Plenum Press, New York, 1985 , p. 1.

[86] J. R. Nix and W. J. Swiatecki, "Studies in the liquiddrop theory of nuclear fission", Nucl. Phys. 71, 1 (1965).

[87] M. G. Itkis, Yu. A. Muzychka, Yu. Ts. Oganessian, V. N. Okolovich, V. V. Pashkevich, A. Ya. Rusanov, V. S. Salamatin, G. N. Smirenkin, and G. G. Chubarian, "Fission of excited nuclei with $Z^{2} / A=20$ - 33: massenergy distributions of fragments, angular momentum, and liquid-drop model", Phys. At. NuCl. 58, 2026 (1995).

[88] A. Ya. Rusanov, V. V. Pashkevich, and M. G. Itkis, "Asymmetric fission barriers for hot rotating nuclei and experimental mass distributions of fission fragments", Phys. Aт. NuCl. 62, 547 (1999).

[89] F.-J. Hambsch, F. Vives, P. Siegler, and S. Oberstedt, "Study of the ${ }^{237} \mathrm{~Np}(\mathrm{n}, \mathrm{f})$-reaction at $\mathrm{MeV}$ neutron energies", NuCl. Phys. A 679, 3 (2000).

[90] H. Nifenecker, "A dynamical treatment of isobaric widths in fission : An example of frozen quantal fluctuations", J. Physique LetT. 41, 47 (1980).

[91] M. Asghar, "Charge distribution in fission - a quantum mechanical phenomenon", Z. PHYs. A 296, 79 (1980).

[92] M. Mirea, "Time-dependent pairing equations for seniority-one nuclear systems", PHYs. REV. C 78, 044618 (2008).

[93] M. Mirea, "New dynamical pair breaking effect", PHYS. LETT. B 680, 316 (2009).

[94] M. Asghar and R. W. Hasse, "Saddle-to-scission landscape in fission: experiments and theories", J. PHYs. Colloques 45, C6-455 (1984).

[95] H. J. Krappe, "Cluster model versus Fermi-fluid approach to fission theory", InT. J. MoD. Phys. E 16, 396 (2007).

[96] W. D. Myers and W. J. Swiatecki, "The congruence energy: a contribution to nuclear masses, deformation energies and fission barriers", NuCl. Phys. A 612, 249 (1997).

[97] The degeneracy of magnetic sub-states is not considered, because it contributes very little to the variation of the density of states as a function of excitation energy.
[98] M. Guttormsen, R. Chankova, M. Hjorth-Jensen, J. Rekstad, S. Siem, A. Schiller, and D. J. Dean, "Free energy and criticality in the nucleon pair breaking process", Phys. Rev. C 68, 034311 (2003).

[99] A. Schiller, A. Bjerve, M. Guttormsen, M. HjorthJensen, F. Ingebretsen, E. Melby, S. Messelt, J. Rekstad, S. Siem, and S. W. Odegard, "Critical temperature for quenching of pair correlations", PHYs. REV. C 63, 021306 (2001).

[100] U. Agvaanluvsan, A. Schiller, J. A. Becker, L. A. Bernstein, P. E. Garrett, M. Guttormsen, G. E. Mitchell, J. Rekstad, S. Siem, A. Voinov, and W. Younes, "Level densities and gamma-ray strength functions in 170,171,172 Yb", Phys. Rev. C 70, 054611 (2004).

[101] A. Bürger, A. C. Larsen, S. Hilaire, M. Guttormsen, S. Harissopulos, M. Kmiecik, T. Konstantinopoulos, M. Krticka, A. Lagoyannis, T. Loennroth, K. Mazurek, M. Norrby, H. T. Nyhus, G. Perdikakis, S. Siem, A. Spyrou, and N. U. H. Syed, "Nuclear level density and gamma-ray strength function of ${ }^{43} \mathrm{Sc} "$, PHYS. REV. C 85, 064328 (2012).

[102] A. C. Larsen, M. Guttormsen, R. Chankova, F. Ingebretsen, T. Loennroth, S. Messelt, J. Rekstad, A. Schiller, S. Siem, N. U. H. Syed, and A. Voinov, "Nuclear level densities and gamma-ray strength functions in ${ }^{44,45}$ Sc", Phys. Rev. C 76, 044303 (2007).

[103] N. U. H. Syed, A. C. Larsen, A. Brger, M. Guttormsen, S. Harissopulos, M. Kmiecik, T. Konstantinopoulos, M. Krticka, A. Lagoyannis, T. Lnnroth, K. Mazurek, M. Norby, H. T. Nyhus, G. Perdikakis, S. Siem, and A. Spyrou, "Extraction of thermal and electromagnetic properties in ${ }^{45}$ Ti", Phys. Rev. C 80, 044309 (2009).

[104] M. Guttormsen, A. C. Larsen, A. Buerger, A. Goergen, S. Harissopulos, M. Kmiecik, T. Konstantinopoulos, M. Krticka, A. Lagoyannis, T. Loennroth, K. Mazurek, M. Norrby, H. T. Nyhus, G. Perdikakis, A. Schiller, S. Siem, A. Spyrou, N. U. H. Syed, H. K. Toft, G. M. Tveten, and A. Voinov, "Fermi's golden rule applied to the gamma decay in the quasicontinuum of ${ }^{46}$ Ti", Phys. REv. C 83, 014312 (2011).

[105] A. C. Larsen, R. Chankova, M. Guttormsen, F. Ingebretsen, S. Messelt, J. Rekstad, S. Siem, N. U. H. Syed, S. W. Oedegard, T. Loennroth, A. Schiller, and A. Voinov, "Microcanonical entropies and radiative strength functions of ${ }^{50,51}$ V", PHYs. REV. C 73, 064301 (2006).

[106] K.-H. Schmidt and B. Jurado, "Final excitation energy of fission fragments", PHys. REv. C 83, 061601 (2011).

[107] R. Müller, A. A. Naqvi, F. Käppeler, and F. Dickmann, "Fragment velocities, energies and masses from fast neutron induced fission of ${ }^{235}$ U", PHys. REV. C 29, 885 (1984)

[108] J. R. Nix, "The normal modes of oscillation of a uniformly charged drop about its saddle-point shape", Ann. Phys. 41, 52 (1967).

[109] N. Dubray, H. Goutte, and J.-P. Delaroche, "Structure properties of ${ }^{226} \mathrm{Th}$ and ${ }^{256,258,260} \mathrm{Fm}$ fission fragments: Mean-field analysis with the Gogny force", PHYs. REV. C 77, 014310 (2008).

[110] J. R. Nix, J. Sierk, H. Hofmann, F. Scheuter, and D. Vautherin, "Stationary Fokker-Planck equation applied to fission dynamics", Nucl. Phys. A 424, 239 (1984).

[111] S. V. Zhdanov, M. G. Itkis, S. I. Mulgin, V. N. Okolovich, A. Ya. Rusanov, G. N. Smirenkin, and M. I. 
Subbotin, "Fragments Energy Distributions and Fission Dynamics of the Heated Nuclei II", Phys. At. NuCL. 56, 175 (1993).

[112] M. Caamano, F. Rejmund, and K.-H. Schmidt, "Evidence for the predominant influence of the asymmetry degree of freedom on the even-odd structure in fissionfragment yields", J. Phys. G: Nucl. PArt. Phys. 38, 035101 (2011).

[113] B. Jurado, K.-H. Schmidt, "Influence of complete energy sorting on the characteristics of the odd-even effect in fission-fragment element distributions", J. PHYS. G: Nucl. Part. Phys. 42, 055101 (2015).

[114] M. V. Ricciardi, A. V. Ignatyuk, A. Kelic, P. Napolitani, F. Rejmund, K.-H. Schmidt, and O. Yordanov, "Complex nuclear-structure phenomena revealed from the nuclide production in fragmentation reactions", NUCL. Phys. A 733, 299 (2004).

[115] M. V. Ricciardi, K.-H. Schmidt, and A. Kelic-Heil, "Even-odd effect in multifragmentation products: the footprints of evaporation", arXiv:1007.0386v1 [nucl-ex] (2010).

[116] B. Mei, H. S. Xu, X. L. Tu, Y. H. Zhang, Yu. A. Litvinov, K.-H. Schmidt, M. Wang, Z. Y. Sun, X. H. Zhou, Y. J. Yuan, M. V. Ricciardi, A. Kelic-Heil, R. Reifarth, K. Blaum, R. S. Mao, Z. G. Hu, P. Shuai, Y. D. Zang, Y. W. Ma, X. Y. Zhang, J. W. Xia, G. Q. Xiao, Z. Y. Guo, J. C. Yang, X. H. Zhang, X. Xu, X. L. Yan, W. Zhang, W. L. Zhan "Origin of odd-even staggering in fragment yields: Impact of nuclear pairing and shell structure on the particle-emission threshold energy", Phys. REV. C 89, 054612 (2014).

[117] L. G. Moretto, "Statistical description of a paired nucleus with the inclusion of angular momentum", NuCL. Phys. A 185, 145 (1972).

[118] D. Rochman, H. Faust, I. Tsekhanovich, F. Gönnenwein, F. Storrer, S. Oberstedt, and V. Sokolov, "Isotopic yields from the reaction ${ }^{245} \mathrm{Cm}\left(\mathrm{n}_{\mathrm{th}}, \mathrm{f}\right)$ at the Lohengrin mass separator", NuCL. PHys. A 710, 3 (2002).

[119] Note that the potential is defined as the binding energy of the nucleus in the respective shape at excitation energy zero. The driving force $F$ in a stochastic process is given by the derivative of the entropy with respect to the collective coordinate $q$ times the temperature: $F=T d S / d q[120]$, not by the potential. The concept of an excitation-energy-dependent potential-energy surface is only an effective way to express the properties of the level density above the potential.

[120] I. I. Gontchar, P. Fröbrich, N. I. Pischasov, "Consistent dynamical and statistical description of fission of hot nuclei", Phys. Rev. C 47, 2228 (1993).

[121] W. J. Swiatecki, "Systematics of spontaneous fission half-lives", Phys. REv. 100, 937 (1955).

[122] Z. Patyk, A. Sobiczewski, P. Armbruster, and K.-H. Schmidt, "Shell effects in the properties of the heaviest nuclei", Nucl. Phys. A 491, 267 (1989).

[123] G. Vladuca, A. Tudora, F.-J. Hambsch, S. Oberstedt, and I. Ruskov, "Evaluation of the fission cross-section within the multi-modal fission approach for ${ }^{235} \mathrm{U}(\mathrm{n}, \mathrm{f})$ ", NuCl. Phys. A 720, 274 (2003).

[124] J. R. Huizenga and R. Vandenbosch, "Interpretation of isomeric cross-section ratios for (n,gamma) and (gamma,n) reactions", Phys. Rev. 120, 1305 (1960).

[125] R. Vandenbosch and J. R. Huizenga, "Method for deducing the angular-momentum distibution from isomeric ra- tios", Phys. Rev. 120, 1313 (1960).

[126] H. Naik, S. P. Dange, R. J. Singh, and A. V. R. Reddy, "Single-particle spin effect on fission fragment angular momentum", Eur. Phys. J. A 31, 195 (2007).

[127] V. M. Strutinski, L. V. Groshev, and M. K. Akimova, "Spectra of gamma-rays from neutron capture by heavy nuclei (II)", Nucl. Phys. 16, 657 (1960).

[128] M. Zielinska-Pfabe and K. Dietrich, "Angular momentum distribution of fission fragments as a result of bending modes at the scission point", Phys. LETT. B 49, 123 (1974).

[129] J. O. Rasmussen, W. Nörenberg, and H. J. Mang, "A model for calculating the angular momentum distribution of fission fragments", NuCL. PHYs. A 136, 465 (1969).

[130] L. G. Moretto, G. F. Peaslee, and G. J. Wozniak, "Angular-momentum-bearing modes in fission", NuCL. Phys. A 502, 453c (1989).

[131] F. Gönnenwein, I. Tsekhanovich, and V. Rubchenya, "Angular momentum of near-spherical fission fragments", Intern. J. Mod. Phys. E 16, 410 (2007).

[132] L. Bonneau, P. Quentin, and I. N. Mikhailov, "Scission configurations and their implication in fission-fragment angular momenta", PHYs. Rev. C 75, 064313 (2007).

[133] M. M. Hoffman, "Torques by electrostatic repulsion", Phys. Rev. 133, B 174 (1964).

[134] S. G. Kadmensky, "Quantum properties of deformation modes of fissile-nucleus motion", Phys. Atom. Nuclei 71, 1193 (2008).

[135] R. W. Hasse and W. D. Myers, "Geometrical Relationships of Macroscopic Nuclear Physics", Springer, Berlin, 1988.

[136] N. Imanishi, I. Fujiwara, and T. Nishi, "Independent isomer yields of $\mathrm{Sb}$ and Te isotopes in thermal-neutron fission of ${ }^{233} \mathrm{U},{ }^{235} \mathrm{U}$ and ${ }^{239} \mathrm{Pu}$ ", NuCl. PHYs. A 263, 141 (1976).

[137] We would also expect an even-odd staggering of the fragment angular momentum in neutron number. However, this effect is not easily observable, because it is washed out by the fluctuations in the prompt-neutron emission.

[138] B. S. Tomar, R. Tripathi, and A. Goswami, "Odd-even effect in fragment angular momentum in low-energy fission of actinides", Pramana 68, 111 (2007).

[139] Th. Rubehn, K. X. Jing, L. G. Moretto, L. Phair, K. Tso, and G. J. Wozniak, "Scaling laws in ${ }^{3}$ He induced nuclear fission", Phys. Rev. C 54, 3062 (1996).

[140] I. Dostrovsky, Z. Fraenkel, and G. Friedlander, "Monte Carlo calculations of nuclear evaporation processes, III. Applications to low-energy reactions", PHYs. REv. 116, 683 (1959).

[141] A. R. Junghans, G. Rusev, R. Schwengner, A. Wagner, and E. Grosse, "Photon data shed new light upon the GDR spreading width in heavy nuclei", Phys. Lett. B 670, 200 (2008).

[142] I. Stetcu, P. Talou, T. Kawano, and M. Jandel, "Isomer production ratios and the angular momentum distribution of fission fragments", Phys. Rev. C 88, 044603 (2013)

[143] O. Litaize, O. Serot, D. Regnier, and C. Manailescu, "Investigation of $\mathrm{n}+{ }^{238} \mathrm{U}$ fission observables", NUCL. Datat SheEts 118, 216 (2014).

[144] M. A. J. Mariscotti, G. Scharf-Goldhaber, and B. Buch, "Phenomenological analysis of ground-state bands in even-even nuclei", PHys. REv. 178, 1864 (1969). 
[145] J. S. Batra and Raj K. Gupta, "Reformulation of the variable moment of inertia model in terms of nuclear softness", Phys. Rev. C 43, 1725 (1991).

[146] D. G. Madland and T. R. England, "The influence of isomeric states on independent fission product yields", NuCl. SCI. EnG. 64, 859 (1977).

[147] G. Rudstam, "Isomeric yields in fission", Proc. Specialists' Meeting on Fission Product Nuclear Data, Tokai, Japan, May 25-27, 1992, NEA/NSC/DOC(92)9.

[148] J. J. Griffin, "Statistical Model of Intermediate Structure", Phys. Rev. Lett. 17, 478 (1966).

[149] M. Blann, "Preequilibrium decay", Ann. Rev. Nucl. SCI. 25, 123 (1975).

[150] M. Blann and M. B. Chadwick, "Precompound MonteCarlo model for cluster induced reactions", PHYs. REV. C 62, 034604 (2000).

[151] C. Kalbach, "Missing final states and the spectral endpoint in exciton model calculations", PHYS. REV. C 73, 024614 (2006).

[152] A. V. Ignatyuk, V. M. Maslov, and A. B. Pashchenko, "Consistent analysis of cross sections on $(n, f)$ and $(n, x n)$ reactions for the actinides", Sov. J. Nucl. Phys. 47, 224 (1988).

[153] B. B. Back, H. Esbensen, C. L. Jiang, and K. E. Rehm, "Recent developments in heavy-ion fusion reactions", Rev. Mod. Phys. 86, 317 (2014).

[154] P. N. Nadtochy, E. G. Ryabov, A. E. Gegechkori, Yu. A. Anischenko, A. D. Adeev, "Four-dimensional Langevin dynamics of heavy-ion-induced fission", PHys. REv. C 85, 064619 (2012).

[155] W. D. Myers and W. J. Swiatecki, "Nuclear masses and deformations", NuCl. PHYs. 81, 1 (1966).

[156] R. Vogt and J. Randrup, "Neutron angular correlations in spontaneous and neutron-induced fission", PHYs. REv. C 90, 064623, (2014).

[157] V. M. Kupriyanov, K. K. Istekov, B. I. Fursov, and G. N. Smirenkin, "Simple description of the dependence of the fission barriers and the ratio gamma(n)/gamma(f) on the nucleonic composition for transuranium nuclei", Sov. J. NuCl. Phys. 32, 184 (1980).

[158] B. Back, Ole Hansen, H. C. Britt, and J. D. Garret "Fission of doubly even actinide nuclei induced by direct reactions", Phys. Rev. C 9, 1924 (1974).

[159] B. Back, H. C. Britt, O. Hansen, B. Leroux, and J. D. Garrett, "Fission of odd-A and doubly odd actinide nuclei induced by direct reactions", PHYs. REV. C 10, 1948 (1974).

[160] A. Gavron, H. C. Britt, E. Konecny, J. Weber, and J. B. Wilhelmy, "Measurement and interpretation of Gamma(n)/Gamma(f) for actinide nuclei", PHYs. REv. LETT. 34, 827 (1975).

[161] A. Gavron, H. C. Britt, E. Konecny, J. Weber, and J. B. Wilhelmy, "Gamma(n)/Gamma(f) for actinide nuclei using $\left({ }^{3} \mathrm{He}, \mathrm{df}\right)$ and $\left({ }^{3} \mathrm{He}, \mathrm{tf}\right)$ reactions", Phys. Rev. C 13, 2374 (1976).

[162] A. Gavron, H. C. Britt, P. D. Goldstone, J. B. Wilhelmy, and S. E. Larsson, "Complexity of the potential-energy surface for fission of ${ }^{238} \mathrm{U}$ ", Phys. Rev. Lett. 38, 1457 (1977).

[163] E. Cheifetz, H. C. Britt, and J. B. Wilhelmy, "Fission probabilities for actinide nuclei excited by the $\left({ }^{12} \mathrm{C}\right.$, ${ }^{8}$ Be $_{\text {g.s. }}$ ) reaction", Phys. Rev. C 24, 519 (1981).

[164] C. Rodriguez-Tajes, F. Farget, X. Derkx, M. Caamano, O. Delaune, K.-H. Schmidt, E. Clement, A. Dijon, A.
Heinz, T. Roger, L. Audouin, J. Benlliure, E. Casarejos, D. Cortina, D. Dore, B. Fernandez-Dominguez, B. Jacquot, B. Jurado, A. Navin, C. Paradela, D. Ramos, P. Romain, M. D. Salsac, and C. Schmitt, "Transfer reactions in inverse kinematics: An experimental approach for fission investigations", PHys. REv. C 89, 024614 (2014)

[165] K.-H. Schmidt and B. Jurado, "Prompt-neutron and prompt-gamma emission from a general description of the fission process", JEF/DOC 1423, NEA of OECD, Paris, 2011, available from 75].

[166] K.-H. Schmidt and B. Jurado, "Global view on fission observables - new insights and new puzzles", PHYs. Proc. 31, 147 (2012).

[167] E. A. C. Crough, "Fission-product yields from neutroninduced fission", At. Data Nucl. Data Tables 19, 417 (1977).

[168] W. E. Stein, "Velocities of fragment pairs from ${ }^{233} \mathrm{U}$, ${ }^{235} \mathrm{U}$, and ${ }^{239} \mathrm{Pu}$ fission", Phys. Rev. 108, 94 (1957).

[169] J. C. D. Milton and J. S. Fraser, "Spontaneous fission fragment velocity measurements and coincident gamma spectra for ${ }^{252}$ Cf", PHYs. REv. 111, 877 (1958).

[170] H. W. Schmitt, W. E. Kiker, and C. W. Williams, "Precision measurements of correlated energies and velocities of ${ }^{252}$ Cf fission fragments", Phys. Rev. 137, B 837 (1965).

[171] E. Moll, H. Schrader, G. Siegert, M. Asghar, J. P. Bocquet, G. Bailleul, J. P. Gautheron, J. Greif, G. I. Crawford, C. Chauvin, H. Ewald, H. Wollnik, P. Armbruster, G. Fiebig, H. Lawin, and K. Sistemich, "Analysis of ${ }^{236}$ U-fission products by the recoil separator 'Lohengrin"', Nucl. Instrum. Methods 123, 615 (1975).

[172] C. Donzaud, S. Czajkowski, P. Armbruster, M. Bernas, C. Böckstiegel, Ph. Dessagne, H. Geissel, E. Hanelt, A. Heinz, C. Kozhoharov, Ch. Miehe, G. Münzenberg, M. Pfützner, W. Schwab, C. Stephan, K. Sümmerer, L. Tassan-Got, and B. Voss, "Low-energy fission investigated in reactions of $750 \mathrm{~A} \mathrm{MeV}{ }^{238} \mathrm{U}$-ions on ${ }^{208} \mathrm{~Pb}$. II: Isotopic distributions", Eur. Phys. J. A 1, 407 (1998).

[173] A. Ebran, J. Taieb, G. Belier, A. Chatillon, B. Laurent, J.-F. Martin, and E. Pellereau, "Picosecond resolution on relativistic heavy ions' time-of-flight measurement", Nucl. Instrum. Methods A 728, 40 (2013).

[174] M. Caamano, O. Delaune, F. Farget, X. Derkx, K.-H. Schmidt, L. Audouin, C.-O. Bacri, G. Barreau, J. Benlliure, E. Casarejos, A. Chbihi, B. Fernandez-Dominguez, L. Gaudefroy, C. Golabek, B. Jurado, A. Lemasson, A. Navin, M. Rejmund, T. Roger, A. Shrivastava, and C. Schmitt, "Isotopic yield distributions of transfer- and fusion-induced fission from ${ }^{238} \mathrm{U}+{ }^{12} \mathrm{C}$ reactions in inverse kinematics", Phys. REv. C 88, 024605 (2013).

[175] R. du Rietz, E. Williams, D. J. Hinde, M. Dasgupta, M. Evers, C. J. Lin, D. H. Luong, C. Simenel, and A. Wakhle, "Mapping quasifission characteristics and timescales in heavy element formation reactions", PHYS. REV. C 88, 054618 (2013).

[176] H. Baba, N. Takahashi, A. Yokoyama, and T. Saito, "Fast fission mechanism and duality of the diffusion process", Eur. Phys. J. A 3, 281 (1998).

[177] I. Gontchar, M. Morjean, and S. Basnary, "Nuclear dissipation from fission time", Europhys. LETT. 57, 355 (2002).

[178] B. Jurado, C. Schmitt, K.-H. Schmidt, J. Benlliure, and A. R. Junghans, "Conditions for the manifestation of 
transient effects in fission", NuCL. PHYs. A 757, 329 (2005).

[179] M. B. Chadwick, M. Herman, P. Oblozinsky, M. E. Dunn, Y. Danon, A. C. Kahler, D. L. Smith, B. Pritychenko, G. Arbanas, R. Arcilla, R. Brewer, D. A. Brown, R. Capote, A. D. Carlson, Y. S. Cho, H. Derrien, K. Guber, G. M. Hale, S. Hoblit, S. Holloway, T. D. Johnson, T. Kawano, B. C. Kiedrowski, H. Kim, S. Kunieda, N. M. Larson, L. Leal, J. P. Lestone, R. C. Little, E. A. McCutchan, R. E. MacFarlane, M. MacInnes, C. M. Mattoon, R. D. McKnight, S. F. Mughabghab, G. P. A. Nobre, G. Palmiotti, A. Palumbo, M. T. Pigni, V. G. Pronyaev, R. O. Sayer, A. A. Sonzogni, N. C. Summers, P. Talou, I. J. Thompson, A. Trkov, R. L. Vogt, S. C. van der Marck, A. Wallner, M. C. White, D. Wiarda, and P. G. Young, "ENDF/B-VII.1 nuclear data for science and technology: cross sections, covariances, fission product yields and decay data", Nucl. Data Sheets 112, 2887 (2011).

[180] P. Schillebeeckx, C. Wagemans, A. J. Deruytter, and R. Barthelemy, "Comparative study of the fragments' mass and energy characteristics in the spontaneous fission of ${ }^{238} \mathrm{Pu},{ }^{240} \mathrm{Pu}$ and ${ }^{242} \mathrm{Pu}$ and in the thermal-neutroninduced fission of ${ }^{239} \mathrm{Pu}$ ", NuCl. Phys. A 545, 623 (1992).

[181] L. Dematte, C. Wagemans, R. Barthelemy, P. D'hondt, and A. Deruytter, "Fragments' mass and energy characteristics in the spontaneous fission of ${ }^{236} \mathrm{Pu},{ }^{238} \mathrm{Pu}$, ${ }^{240} \mathrm{Pu},{ }^{242} \mathrm{Pu}$, and ${ }^{244} \mathrm{Pu}$ ", NuCl. Phys. A 617, 331 (1997).

[182] E. K. Hulet, J. F. Wild, R. J. Dougan, R. W. Lougheed, J. H. Landrum, A. D. Dougan, P. A. Baisden, C. M. Henderson, and R. J. Dupzyk, "Spontaneous fission properties of ${ }^{258} \mathrm{Fm},{ }^{260} \mathrm{Md},{ }^{258} \mathrm{No}$ and ${ }^{260} 104$ : Bimodal fission", Phys. Rev. C 40, 770 (1989).

[183] D. C. Hoffman, D. M. Lee, K. E. Gregorich, M. J. Nurmia, R. B. Chadwick, K. B. Chen, K. R. Czerwinski, C. M. Gannett, H. L. Hall, R. A. Henderson, B. Kadkodayan, S. A. Kreek, and J. D. Leyba, "Spontaneous fission properties of 2.9-s ${ }^{256}$ No", PhYs. ReV. C 41, 631 (1990).

[184] T. M. Hamilton, K. E. Gregorich, D. M. Lee, K. R. Czerwinski, N. J. Hannink, C. D. Kacher, B. Kadkhodayan, S. A. Kreek, M. J. Nurmia, M. R. Lane, M. P. Neu. A. Türler, and D. C. Hoffman, "Spontaneous fission properties of ${ }^{259}$ Lr", Phys. Rev. C 46, 1873 (1992).

[185] M. R. Lane, K. E. Gregorich, D. M. Lee, M. F. Mohar, M. Hsu, C. D. Kacher, B. Kadkhodayan, M. P. Neu, N. J. Stoyer, E. R. Sylwester, J. C. Yang, and D. C. Hofmann, "Spontaneous fission properties of ${ }^{252} \mathrm{Rf}$ ", PHYs. REV. C 53, 2893 (1996).

[186] U. Quade, K. Rudolph, S. Skorka, P. Armbruster, H.G. Clerc, W. Lang, M. Mutterer, C. Schmitt, J. P. Theobald, G. Gönnenwein, J. Pannicke, H. Schrader, G. Siegert, and D. Engelhardt, "Nuclide yields of light fission products from thermal-neutron induced fission of ${ }^{233} \mathrm{U}$ at different kinetic energies", NuCL. PHYs. A 487, 1 (1988).

[187] Jie Zhao, Bing-Nan Lu, Dario Vretenar, En-Guang Zhao, and Shan-Gui Zhou, "Multidimensionally constrained relativistic mean-field study of triple-humped barriers in actinides", Phys. Rev. C 91, 014321 (2015).

[188] L. Csige, D. M. Filipescu, T. Glodariu, J. Gulyas, M. M. Günther, D. Habs, H. J. Karwowski, A. Krasznahorkay,
G. C. Rich, M. Sin, L. Stroe, O. Tesileanu, and P. G. Thirolf, "Exploring the multihumped fission barrier of ${ }^{238} \mathrm{U}$ via sub-barrier photofission", PHYs. REV. C 87, 044321 (2013).

[189] C. Schmitt, A. Guessous, J. P. Bocquet, H.-G. Clerc, R. Brissot, D. Engelhardt, H. R. Faust, F. Gönnenwein, M. Mutterer, H. Nifenecker, J. Pannicke, Ch. Ristori, and J. P. Thoebald, "Fission yields at different fission-product kinetic energies for the thermal-neutron-induced fission of ${ }^{239} \mathrm{Pu} "$, Nucl. Phys. A 430, 21 (1984).

[190] M. Djebara, M. Asghar, J. P. Bocquet, R. Brissot, J. Crancon, Ch. Ristori, E. Aker, D. Engelhardt, J. Gindler, B. D. Wilkins, U. Quade, and K. Rudolph, "Mass and nuclear-charge yields for ${ }^{249} \mathrm{Cf}\left(\mathrm{n}_{\mathrm{th}}, \mathrm{f}\right)$ at different fission-product kinetic energies", NuCL. PHYs. A 496, 346 (1989).

[191] R. Hentzschel, H. R. Faust, H. O. Denschlag, B. D. Wilkins, and J. Gindler, "Mass, charge and energy distributions in the very asymmetric fission of ${ }^{249} \mathrm{Cf}$ induced by thermal neutrons", NuCL. PHYs. A 571, 427 (1994).

[192] C. Budtz-Joergensen and H.-H. Knitter, "Simultaneous investigation of fission fragments and neutrons in ${ }^{252}$ Cf(sf)", Nucl. Phys. A 490, 307 (1988).

[193] IAEA Tecdoc 1168, "Compilation and evaluation of fission yield nuclear data", IAEA, Vienna, 2000.

[194] L. E. Glendenin, J. E. Gindler, D. J. Henderson, and J. W. Meadows, "Mass distributions for monoenergeticneutron-induced fission of ${ }^{235}$ U", PHYs. REV. C 24, 2600 (1981).

[195] J. E. Gindler, L. E. Glendenin, D. J. Henderson, and J. W. Meadows, "Mass distributions in monoenergeticneutron-induced fission of ${ }^{239} \mathrm{Pu}$ ", Phys. REV. C 27, 2058 (1983).

[196] L. E. Glendenin, J. E. Gindler, J. Ahmad, D. J. Henderson, and J. W. Meadows, "Mass distribution in monoenergetic-neutron-induced fission of ${ }^{232}$ Th", PHYS. REV. C 22, 152 (1980).

[197] S. Nagy, K. F. Flynn, J. E. Gindler, J. W. Meadows, and L. E. Glendenin, "Mass distributions in monoenergeticneutron-induced fission of ${ }^{238}$ U", PHYs. REV. C 17, 163 (1968).

[198] G. P. Ford and R. B. Leachman, "Fission Mass Yield Dependence on Angular Momentum", Phys. Rev. 137, B 826 (1965).

[199] J. Laurec, A. Adam, T. de Bruyne, E. Bauge, T. Granier, J. Aupiais, O. Bersillon, G. Le Petit, N. Authier, and P. Casoli, "Fission Product Yields of ${ }^{233} \mathrm{U}$, ${ }^{235} \mathrm{U},{ }^{238} \mathrm{U}$ and ${ }^{239} \mathrm{Pu}$ in Fields of Thermal Neutrons, Fission Neutrons and 14.7-MeV Neutrons", NuCL. DATA SheETs 111, 2965 (2010).

[200] M. Mac Innes, M. B. Chadwick, and T. Kawano, "Fission Product Yields for $14 \mathrm{MeV}$ Neutrons on ${ }^{235} \mathrm{U},{ }^{238} \mathrm{U}$ and ${ }^{239} \mathrm{Pu}$ ", NuCl. DATA Sheets 112, 3135 (2011) and references therein.

[201] T. C. Chapman, G. A. Anzelon, G. C. Spitale, and D. R. Nethaway, "Fission product yields from 6-9 MeV neutron-induced fission of ${ }^{235} \mathrm{U}$ and ${ }^{238} \mathrm{U}$ ", PHYs. REV. C 17, 1089 (1978).

[202] H. Naik, S. P. Dange, R. J. Singh, and T. Datta, "Systematics of fragment angular momentum in low-energy fission of actinides", NuCl. PHYs A 587, 273 (1995).

[203] H. Naik, S. P. Dange, and R. J. Singh, "Angular momentum of fission fragments in low energy fission of ac- 
tinides", Phys. Rev. C 71, 014304 (2005).

[204] A. Bail, O. Serot, L. Mathieu, O. Litaize, T. Materna, U. Koester, H. Faust, A. Letourneau, and S. Panebianco, "Isotopic yield measurement in the heavy mass region for ${ }^{239} \mathrm{Pu}$ thermal neutron induced fission", PHYs. REV. C 84, 034605 (2011).

[205] H. Naik, S. P. Dange, and R. J. Singh, "Fission fragment angular momentum in odd-Z fissioning systems", EuR. Phys. J. A 7, 377 (2000).

[206] H. Naik, S. P. Dange, D. J. Singh, S. K. Das, and R. Guin, "Some important aspects of fragment angular momentum in medium energy fission of ${ }^{238} \mathrm{U}$ ", NUCL. Phys. A 648 (1999) 45.

[207] A. L. Nichols, "Nuclear data requirements for decay heat calculations", Lectures given at the Workshop on Nuclear Reaction Data and Nuclear Reactors: Physics, Design and Safety, Trieste, 2002, IAEA, Vienna, Austria.

[208] T. Datta, S. P. Dange, A. G. C. Nair, Satya Prakash, and M. V. Ramaniah, "Fission fragment angular momentum: Ratios of independent yields of isomers of ${ }^{95} \mathrm{Nb}$ and ${ }^{132} \mathrm{I}$ in thermal-neutron-induced fission of ${ }^{233} \mathrm{U}$ ", Phys. Rev. C 25, 358 (1982).

[209] G. P. Ford, K. Wolfsberg, and B. R. Erdal, "Independent yields of the isomers of ${ }^{133} \mathrm{Xe}$ and ${ }^{135} \mathrm{Xe}$ for neutroninduced fission of ${ }^{233} \mathrm{U},{ }^{235} \mathrm{U},{ }^{238} \mathrm{U}$, and ${ }^{242} \mathrm{Am}{ }^{\mathrm{m}}$ ", PHYs. REV. C 30, 195 (1984).

[210] H. Thierens, B. Proot, D. De Frenne, and E. Jacobs, "Independent isomeric yield ratio of ${ }^{134} \mathrm{I}$ in the photofission of ${ }^{235} \mathrm{U}$ and ${ }^{238} \mathrm{U} "$, Phys. REv. C 25, 1546 (1982).

[211] D. Regnier, private communication (2015).

[212] E. K. Hulet, J. F. Wild, R. J. Dougan, R. W. Lougheed, J. H. Landrum, A. D. Dougan, M. Schädel, R. L. Hahn, P. A. Baisden, C. M. Henderson, R. J. Dupzyk, K. Sümmerer, and G. R. Bethune, "Bimodal Symmetric Fission Observed in the Heaviest Elements", PHYs. REV. LeTt. 56, 313 (1986).

[213] V. V. Malinovskij, V. G. Vorob'eva, and B. D. Kuz'minov, "Review of measurements of the average number of prompt neutrons", Report INDC(CCP)-239, IAEA, Vienna, Austria, 1985.

[214] R. W. Mills, "Fission product yield evaluation", PhD thesis, university of Birmingham, 1995.

[215] F. Manero, V. A. Konshin, "Status of the energydependent $\nu$-values for the heavy isotopes $(Z>90)$ from thermal to $15 \mathrm{MeV}$ and $\nu$-values for spontaneous fission", Atomic Energy Rev. 10, 637, IAEA, Vienna, Austria, 1972.

[216] C. Budtz-Jorgensen and H.-H. Knitter, "Simultaneous investigation of fission fragments and neutrons in ${ }^{252}$ Cf(sf)", Nucl. Phys. A 490, 307 (1988).

[217] M. S. Zucker and N. E. Holden, "Energy Dependence of Neutron Multiplicity in Fast-Neutron-Induced Fission for ${ }^{235,238} \mathrm{U}$ and ${ }^{239} \mathrm{Pu}$ ", Report BNL-38491 (1986).

[218] E. J. Axton, "Evaluation of the thermal neutron constants of ${ }^{233} \mathrm{U},{ }^{235} \mathrm{U},{ }^{239} \mathrm{Pu}$ and ${ }^{241} \mathrm{Pu}$ and the fission neutron yield of ${ }^{252}$ Cf", in TECDOC 335, Nuclear Standard Reference Data, p. 214, IAEA, Vienna, 1985.

[219] N. V. Kornilov, F.-J. Hambsch, I. Fabry, S. Oberstedt, T. Belgya, Z. Kis, L. Szentmiklosi, and S. Simakov, "The ${ }^{235} \mathrm{U}(\mathrm{n}, \mathrm{f})$ prompt fission neutron spectrum at $100 \mathrm{~K}$ input neutron energy", NuCl. SCIEnce Engin. 165, 117 (2010).

[220] W. Mannhart, "Physics of neutron emission in fission", INDC(NDS)-220 (1989) 305, IAEA, Vienna.
[221] V. P. Eismont, "Neutrons from fission of excited nuclei", Atomn. Ener. 19, 113 (1965).

[222] V. M. Maslov, Yu V. Porodzinskij, M. Baba, A. Hasegawa, N. Kornilov, A. B. Kagalenko, and N. A. Tetereva, "Prompt fission neutron spectra of ${ }^{238} \mathrm{U}(\mathrm{n}, \mathrm{f})$ above emissive fission threshold", Eur. Phys. J. A 18, $93(2003)$.

[223] N. V. Kornilov, A. B. Kagalenko, S. V. Poupko, P. A. Androsenko, and F. -J. Hambsch, "New evidence of an intense scission neutron source in the ${ }^{252} \mathrm{Cf}$ spontaneous fission", NuCl. Phys. A 686, 187 (2001).

[224] G. V. Danilyan, T. Wilpert, P. Granz, V. A. Krakhotin, F. Mezei, V. V. Novitsky, V. S. Pavlov, M. V. Russina, and P. B. Shatalov, "Angular correlations in emission of prescission neutrons from ${ }^{235} \mathrm{U}$ fission induced by slow polarized neutrons", Phys. Aтом. Nucl. 71, 2003 (2008).

[225] G. A. Petrov, A. M. Gagarski, I. S. Guseva, V. E. Sokolov, G. V. Val'ski, A. S. Vorobiev, D. O. Krinitcin, O. A. Shcherbakov, D. V. Nikolaev, Yu. S. Pleva, and T. A. Zavarukhina, "Basic results of investigations of scission neutrons in nuclear fission at low excitation energies", Phys. Aтом. Nucl. 71, 1137 (2008).

[226] N. Carjan and M. Rizea, "Scission neutrons and other scission properties as function of mass asymmetry in ${ }^{235} \mathrm{U}\left(\mathrm{n}_{\mathrm{th}}, \mathrm{f}\right) "$, Phys. ReV. C 82, 014617 (2010).

[227] A. A. Bojcov, A. F. Semenov, and B. I. Starostov, "Relative measurements of the spectra of prompt fission neutrons for thermal neutron fission of the nuclei ${ }^{233} \mathrm{U},{ }^{235} \mathrm{U}$, ${ }^{239} \mathrm{Pu}$ in the energy range $0.01-5 \mathrm{MeV} ", 6$ th All Union Conf. on Neutron Physics, Kiev (1983) Vol. 2, p. 294.

[228] A. Lajtai, J. Kecskemeti, J. Safar, and P. P. Dyachenko, "Prompt neutron spectra for energy range $30 \mathrm{keV}-4$ $\mathrm{MeV}$ from fission of ${ }^{233} \mathrm{U},{ }^{235} \mathrm{U},{ }^{239} \mathrm{Pu}$ induced by thermal neutrons", Nucl. Data Conf., Santa Fe (1985) Vol. 1, p. 613.

[229] L. V. Drapchinsky et al., communicated by N. Capote, 2012 .

[230] A. S. Vorobyev, O. A. Shcherbakov, Yu. S. Pleva, A. M. Gagarski, G. V. Val'ski, G. A. Petrov, V. I. Petrova, and T. A. Zavarukhina, "Measurements of angular and energy distributions of prompt neutrons from thermal neutron-induced fission", NuCl. Instrum. Methods A 598, 795 (2009)

[231] J. W. Boldeman, A. R. de L. Musgrover, R. L. Walsh, "Prompt neutrons from ${ }^{236} \mathrm{U}$ fission fragments", AUST. J. Phys. 24, 821 (1971).

[232] A. S. Vorobyev, O. A. Shcherbakov, Yu. S. Pleva, A. M. Gagarski, G. V. Val'ski, G. A. Petrov, V. I. Petrova, T. A. Zavarukhina, I. S. Guseva, V. E. Sokolov, D. O. Krinitzin, and D. V. Nikolaev, "Angular and energy distributions of prompt neutrons from thermal neutron-induced fission of ${ }^{233} \mathrm{U},{ }^{235} \mathrm{U}(\mathrm{n}, \mathrm{F})$ ", Conf: Int.Sem.on Interactions of Neutrons with Nuclei, No.17, p.60 (2010).

[233] N. V. Kornilov, private communation, 20. 4. 2012.

[234] F.-J. Hambsch and S. Oberstedt, "Investigation of the far asymmetric region in ${ }^{252} \mathrm{Cf}(\mathrm{sf})$ ", NuCl. PHYs. A 617, 347 (1997).

[235] B. E. Watt, "Energy Spectrum of Neutrons from Thermal Fission of ${ }^{235}$ U", Phys. Rev. 87, 103 (1952).

[236] D. Madland and J. Nix, "New calculation of prompt fission neutron spectra and average prompt neutron multiplicities", NuCl. SCI. ENG. 81, 213 (1982). 
[237] D. G. Madland, R. J. LaBauve, and J. R. Nix, "Recent improvements in the calculation of prompt fission neutron spectra: preliminary results", 1989, IAEAINDC(NDS)-220, 259, IAEA, Vienna, Austria.

[238] S. Lemaire, P. Talou, T. Kawano, M. B. Chadwick, and D. G. Madland, "Monte Carlo approach to sequential neutron emission from fission fragments", PHYS. REV. C 72, 024601 (2005).

[239] A. Tudora and F.-J. Hambsch, "Point by Point model calculation of the prompt neutron multiplicity distribution for spontaneous and neutron induced fission of actinides", Ann. Nucl. Energy 37, 771 (2010).

[240] R. Vogt, J. Randrup, D. A. Brown, M. A. Descalle, and W. E. Ormand, "Event-by-event evaluation of the prompt fission neutron spectrum from ${ }^{239} \mathrm{Pu}(\mathrm{n}, \mathrm{f})$ ", Phys. Rev. C 85, 024608 (2012).

[241] M. I. Svirin, G. N. Lovchikova, and A. M. Trufanov, "Features of the spectra of neutrons accompanying the neutron-induced emission fission of ${ }^{238}$ U", Phys. АTOM. NuCLEI 60, 727 (1997).

[242] A. M. Trufanov, G. N. Lovchikova, M. I. Svirin, A. V. Polyakov, V. A. Vinogradov, V. D. Dmitriev, and G. S. Boikov, "Investigation of the spectra of neutrons originating from ${ }^{238} \mathrm{U}$ fission induced by 0.5 - and $13.2-\mathrm{MeV}$ neutrons", Phys. Aтom. Nuclei 64, 1 (2001).

[243] G. N. Lovchikova, A. M. Trufanov, M. I. Svirin, V. A. Vinogradov, and A. V. Polyakov, "Spectra and mean energies of prompt neutrons from ${ }^{238} \mathrm{U}$ fission induced by primary neutrons of energy in the region $\mathrm{E}_{n}<20$ MeV", Phys. Atom. Nuclei 67, 1246 (2004).

[244] V. M. Maslov, " ${ }^{239} \mathrm{Pu}$ prompt fission neutron spectra”, AT. ENERGY 103, 633 (2007).

[245] W. Mannhart, "Evaluation of the ${ }^{252} \mathrm{Cf}$ fission neutron spectrum between $0 \mathrm{MeV}$ and $20 \mathrm{MeV}$ ", in proceedings of IAEA Meeting, Leningrad, 1986, IAEA-TECDOC410 (IAEA, Vienna, 1987), p. 158.

[246] M. V. Kornilov, A. B. Kagalenko, and F.-J. Hambsch "Computing the Spectra of Prompt Fission Neutrons on the Basis of a New Systematics of Experimental Data", Phys. Атом. Nuclei 62, 173 (1999).

[247] I. Kodeli, A. Trkov, R. Capote, Y. Nagaya, and V. Maslov, "Evaluation and use of the prompt fission neutron spectrum and spectra covariance matrices in criticality and shielding", Nucl. Instrum. Methods A 610, 540 (2009).

[248] V. M. Maslov, N. A. Tetereva, V. G. Pronyaev, A. B. Kagalenko, K. I. Zolotarev, R. Capote, T. Granier, B. Morillon, F.-J. Hambsch, and J.-C. Sublet, " ${ }^{235} \mathrm{U}(\mathrm{n}, \mathrm{f})$, ${ }^{233} \mathrm{U}(\mathrm{n}, \mathrm{f})$ and ${ }^{239} \mathrm{Pu}(\mathrm{n}, \mathrm{f})$ Prompt Fission Neutron Spectra", J. Korean Phys. Soc. 59, 1337 (2011).

[249] V. Manea and A. Tudora, "Approach for the fission fragment total kinetic energy TKE(A) calculation: Application to prompt neutron emission models", ANN. NUCL. ENERGY 38, 72 (2011).

[250] R. J. Howerton, "nu-bar revised", Nucl. SCI. ENG. 62, 438 (1977).

[251] G. S. Boykov, V. D. Dmitriev, G. A. Kudyaev, V. M. Maslov, Yu. B. Ostapenko, M. I. Svirin, and G. N. Smirenkin, "Peculiarities in the neutron spectra accompanying neutron-induced emission fission of actinide nuclei", Nucl. Energy 21, 585 (1994).

[252] G. Rimpault, A. Courcelle, and D. Blanchet, "Needs for accurate measurements of spectrum and multiplicity of prompt $\gamma$ emitted in fission", (http://www.oecd-nea.org/dbdata/hprl/tmp/ HPRLgammafission.pdf).

[253] A. Oberstedt, private communication (2015).

[254] D. Regnier, "Contribution à l'étude des gammas prompts de fission", $\mathrm{PhD}$ thesis, University Grenoble, France (2013).

[255] F. Pleasonton, R. L. Ferguson, and H. W. Schmitt, "Prompt Gamma Rays Emitted in the ThermalNeutron-Induced Fission of ${ }^{235}$ U", Phys. Rev. C 6, 1023 (1972).

[256] V. V. Verbinski, H. Weber, and R. E. Sund, "Prompt gamma rays from ${ }^{235} \mathrm{U}(\mathrm{n}, \mathrm{f}),{ }^{239} \mathrm{Pu}(\mathrm{n}, \mathrm{f})$, and spontaneous fission of ${ }^{252}$ Cf", Phys. Rev. C 7, 1173 (1973).

[257] Several predictions have been published for the models considered in the Tables XIV to XVI, using different sets of parameters. We present here the set which gives best agreement with experiment. As for the GEF prediction, default parameters have been used; no specific adjustment to these data was done.

[258] S. Oberstedt, T. Belgya, R. Billnert, R. Borcea, T. Brys, W. Geerts, A. Göök, F.-J. Hambsch, Z. Kis, T. Martinez, S. Oberstedt, L. Szentmiklosi, K. Takacs, and M. Vidali, "Improved values for the characteristics of prompt-fission gamma-ray spectra from the reaction ${ }^{235} \mathrm{U}\left(\mathrm{n}_{\mathrm{th}}, \mathrm{f}\right) "$ ", Phys. Rev. C 87, 051602(R) (2013).

[259] B. Becker, P. Talou, T. Kawano, Y. Danon, and I. Stetcu, "Monte Carlo Hauser-Feshbach predictions of prompt fission gamma rays: Application to $\mathrm{n}_{\mathrm{th}}+{ }^{235} \mathrm{U}$, $\mathrm{n}_{\mathrm{th}}+{ }^{239} \mathrm{Pu}$, and ${ }^{252} \mathrm{Cf}(\mathrm{sf}) "$ ", Phys. Rev. C 87, 014617 (2013).

[260] R. Vogt and J. Randrup, "Event-by-event study of photon observables in spontaneous and thermal fission", Phys. Rev. C 87, 044602 (2013).

[261] F. Pleasonton, "Prompt gamma rays emitted in the thermal-neutron induced fission of ${ }^{233} \mathrm{U}$ and ${ }^{239} \mathrm{Pu}$ ", NuCl. Phys. A 213, 413 (1973).

[262] A. Chyzh, C. Y. Wu, E. Kwan, R. A. Henderson, J. M. Gostic, T. A. Bredeweg, A. Couture, R. C. Haight, A. C. Hayes-Sterbenz, M. Jandel, H. Y. Lee, J. M. O'Donnell, and J. L. Ullmann, "Systematics of prompt gamma-ray emission in fission", Phys. Rev. C 87, 034620 (2013).

[263] A. Chyzh, C. Y. Wu, E. Kwan, R. A. Henderson, J. M. Gostic, T. A. Bredeweg, R. C. Haight, A. C. HayesSterbenz, M. Jandel, J. M. ODonnell, and J. L. Ullmann, "Evidence for the stochastic aspect of prompt gamma emission in spontaneous fission", PHYs. REV. C 85, 021601 (2012).

[264] R. Billnert, F.-J. Hambsch, A. Oberstedt, and S. Oberstedt, "New prompt spectral gamma-ray data from the reaction ${ }^{252} \mathrm{Cf}(\mathrm{sf})$ and its implication on present evaluated nuclear data files", PHYs. REV. C 87, 024601 (2013).

[265] S. Oberstedt, R. Billnert, T. Belgya, T. Brys, W. Geerts, C. Guerrero, F.-J. Hambsch, Z. Kis, A. Moens, A. Oberstedt, G. Sibbens, L. Szentmiklosi, D. Vanleeuw, and M. Vidali, "High-precision prompt-gamma-ray spectral data from the reaction ${ }^{241} \mathrm{Pu}\left(\mathrm{n}_{\mathrm{th}}, \mathrm{f}\right)$ "), PHYs. REV. C 90, 024618 (2014).

[266] S. P. Maydanyuk, V. S. Olkhovsky, G. Mandaglio, M. Manganaro, G. Fazio, and G. Giardina, "Bremsstrahlung emission of high energy accompanying spontaneous fission of ${ }^{252}$ Cf", PHYs. REV. C 82, 014602 (2010). 
[267] P. Singer, M. Mutterer, Yu. N. Kopach, M. Klemens, A. Hotzel, D. Schwalm, P. Thirolf, and M. Hesse, "Highenergy gamma-rays in alpha-accompanied spontaneous fission of ${ }^{252}$ Cf", Z. PHYs. A 359, 41 (1997).

[268] D. Pandit, S. Mukhopadhyay, S. Bhattacharya, S. Pal, A. De, and S. R. Banejee, "Coherent bremsstrahlung and GDR width from ${ }^{252}$ Cf cold fission", PHYS. LETT. B 690, 473 (2010).

[269] A. Hotzel, P. Thirolf, Ch. Ender, D. Schwalm, M. Mutterer, P. Singer, M. Klemens, J. P. Theobald, M. Hesse, F. Gönnenwein, and H. v. d. Plög, "High-energy gammarays accompanying the spontaneous fission of ${ }^{252} \mathrm{Cf}$ ", $\mathrm{Z}$. Phys. A 356, 299 (1996).

[270] A. Schiller, L. Bergholt, M. Guttormsen, E. Melby, J. Rekstad, and S. Siem, "Extraction of level density and gamma strength function from primary gamma spectra", Nucl. Instrum. Methods Phys. Res. A 447, 498 (2000).

[271] H. K. Toft, A. C. Larsen, A. Buerger, M. Guttormsen, A. Goergen, H. T. Nyhus, T. Renstroem, S. Siem, G. M. Tveten, and A. Voinov, "Evolution of the pygmy dipole resonance in Sn isotopes", Phys. REv. C 83, 044320 (2011).

[272] Also in other work an inconsistency in the description of prompt neutrons and prompt gammas has been observed. A good reproduction of both the prompt-neutron and the prompt-gamma spectra has been obtained in Ref. 259] by using different adjusted values of the spin cut-off parameter that determines the fragment angularmomentum distribution.

[273] I. Stetcu, P. Talou, T. Kawano, and M. Jandel, "Properties of prompt-fission gamma rays", PHYs. REV. C 90, 024617 (2014).

[274] J. B. Fitzgerald, D. Habs, F. Heller, P. Reiter, D. Schwalm, P. Thirolf, and A. Wiswesser, "Fragment mass dependence of the high-energy gamma-ray spectrum in fission", Z. PHYs. A 355, 401 (196).

[275] H. van der Plög, C. R. Laurens, J. C. S. Bacelar, A. Buda, J. J. Gaardhoje, G. van 't Hof, N. KalantarNayestanaki, A. van der Woude, and Z. Zelazny, "Study of the gamma emission probability accompanying the spontaneous fission of ${ }^{252}$ Cf", NuCl. Phys. A 569, 83 (1994).

[276] Srijit Bhattacharya, Deepak Pandit, Balaram Dey, Debasish Mondal, S. Mukhopadhyay, Surajit Pal, A. De, and S. R. Banerjee, "Examination of level density prescriptions for the interpretation of high-energy gammaray spectra", Phys. REv. C 90, 054319 (2014).

[277] S. A. E. Johansson, "Gamma de-excitation of fission fragments: (I). Prompt radiation", NuCl. Phys. A 60, 378 (1964).

[278] H. Maier-Leibnitz, H. W. Schmitt, and P. Armbruster, "Average number and energy of gamma-rays emitted as a function of fragment mass in ${ }^{235} \mathrm{U}$ thermal-neutroninduced fission", Proc. Symp. Phys. Chem. of Fission, Salzburg, 1965, Vol. II, IAEA Vienna, p. 143, 1965.

[279] H. Albinsson and L. Lindow, "Prompt gamma radiation from fragments in the thermal fission of ${ }^{235} \mathrm{U}$ ", Aktiebolaget Atomenergi Studsvik (Nykoping, Sweden) Technical Report No. AE-398, 1970 (unpublished).

[280] P. Glässel, R. Schmid-Fabian, D. Schwalm, D. Habs, and H. U. v. Helmolt, " ${ }^{252} \mathrm{Cf}$ fission revisited - new insights into the fission process", NuCl. PHYs. A 502, 315c (1989).
[281] K. Skarsvag, "Angular correlation of fission fragments and prompt gamma rays from slow neutron fission of ${ }^{235}$ U", NuCl. Phys. A 96, 385 (1967).

[282] E. Nardi, A. Gavron, and Z. Fraenkel, "Total energy associated with prompt gamma-ray emission in the spontaneous fission of ${ }^{252}$ Cf", PHYs. REv. C 8, 2293 (1973).

[283] A. Bogachev, L. Krupa, O. Dorvaux, E. Kozulin, M. Itkis, M. -G. Porquet, A. Astier, D. Curien, I. Deloncle, G. Duchene, B. J. P. Gall, F. Hanappe, F. Khalfallah, M. Rousseau, L. Stuttge, N. Redon, and O. Stezowski, "Fission fragment properties obtained in the gamma-gamma-gamma coincidence method in the reaction ${ }^{208} \mathrm{~Pb}\left({ }^{18} \mathrm{O}, \mathrm{f}\right) "$, Eur. Phys. J. A 34, 23 (2007).

[284] R. W. Pelle and F. C. Maienschein, "Spectrum of photons emitted in coincidence with fission of ${ }^{235} \mathrm{U}$ by thermal neutrons", Phys. Rev. C 3, 373 (1971).

[285] F. Pühlhofer, "On the interpretation of evaporation residue mass distributions in heavy-ion induced fusion reactions", NuCl. PHYs. 280, 267 (1977).

[286] D. J. Hofman, B. B. Back, C. P. Montoya, S. Schadmand, R. Varma, and P. Paul, "High energy gamma rays from ${ }^{252} \mathrm{Cf}$ spontaneous fission", Phys. Rev. C 47, 1103 (1993).

[287] K. Skarsvag, "Differential angular distribution of prompt gamma rays from spontaneous fission of ${ }^{252} \mathrm{Cf}$ ", Phys. Rev. C 22, 638 (1980).

[288] M. Jandel et al., "Prompt gamma-ray emission in neutron induced fission of ${ }^{235}$ U", LANL Report, LA-UR12-24975 (2013) (unpublished).

[289] R. P. Schmitt, G. Mouchaty, and D. R. Haenni, "Angular momentum transfer in ${ }^{12} \mathrm{C}-,{ }^{20} \mathrm{Ne}$ - and ${ }^{40} \mathrm{Ar}$-induced fission", NuCl. Phys. A 427, 614 (1984).

[290] H. Baba, G. Saito, N. Takahashi, A. Yokoyama, T. Miyauchi, S. Mori, D. Yano, T. Hakoda, K. Takamiya, K. Nikanishi, and Y. Nakagome, "Role of effective distance in the fission mechanism study by the doubleenergy measurement for uranium isotopes", J. NUCL. SCI. TEChN. 34, 871 (1997).

[291] F. Martin, "Étude des distributions en masse, charge nucléaire et énergie cinétique des produits de fission de $\mathrm{l}^{, 233} \mathrm{U}\left(\mathrm{n}_{\mathrm{th}}, \mathrm{f}\right)$ et du ${ }^{241} \mathrm{Pu}\left(\mathrm{n}_{\mathrm{th}}, \mathrm{f}\right)$ mesurées auprès du spectromètre de masse Lohengrin (ILL)", $\mathrm{PhD}$ thesis, University of Grenoble, 2013 (in French).

[292] V. F. Apalin, Yu. N. Gritsyuk, I. E. Kutikov, V. I. Lebedev, and L. A. Mikaelian, "Kinetic energy of fragments and energy balance in the fission of ${ }^{235} \mathrm{U}$ by thermal neutrons", NuCl. Phys. 71, 546 (1965).

[293] A. Bail, "Fission yield measurements. Measurement of mass and isotopic yields of heavy fission fragments: ${ }^{239} \mathrm{Pu}(\mathrm{n}, \mathrm{f}) ", \mathrm{PhD}$ thesis, University of Bordeaux, 2009.

[294] W. Surin, A. I. Sergachev, N. I. Rezchikov, and B. D. Kuz'minov, "Yields and kinetic energies of fragments in the fission of ${ }^{233} \mathrm{U}$ and ${ }^{239} \mathrm{Pu}$ by 5.5 - and $15-\mathrm{MeV}$ neutrons", Sov. J. NuCl. Phys. 14, 523 (1972).

[295] C. Wagemans, E. Allaert, A. Deruytter, R. Barthelemy, and P. Schillebeeckx, "Comparison of the energy and mass characteristics of the ${ }^{239} \mathrm{Pu}\left(\mathrm{n}_{\mathrm{th}}, \mathrm{f}\right)$ and the ${ }^{240} \mathrm{Pu}(\mathrm{sf})$ fragments", Phys. Rev. C 30, 218 (1984).

[296] C. Tsuchiya, Y. Nakagome, H. Yamana, H. Moriyama, K. Nishio, I. Kanno, K. Shin, and I. Kimura, "Simultaneous Measurement of Prompt Neutrons and Fission Fragments for ${ }^{239} \mathrm{Pu}\left(\mathrm{n}_{\mathrm{th}}, \mathrm{f}\right)$ ", J. NuCl. SCIENCE TeChN. 37, 941 (2000). 
[297] W. Holubarsch, E. Pfeiffer, and F. Gönnenwein, "Fragment kinetic energies in $\mathrm{MeV}$ neutron-induced fission of ${ }^{232}$ Th", NuCl. Phys. A 171, 631 (1971).

[298] C. J. D. Milton and J. S. Fraser, "Time-of-flight fission studies on ${ }^{233} \mathrm{U},{ }^{235} \mathrm{U}$ and ${ }^{239} \mathrm{Pu}$ ", CAN. Journ. Phys. 40, 1626 (1962).

[299] C. Wagemans, "The Nuclear Fission Process", 1991, CRC Press, Boca Raton, USA.

[300] N. Varapai, F.-J. Hambsch, S. Oberstedt, O. Serot, G. Barreau, N. Kornilov, and Sh. Zeinalov, "Development of a digital technique for the determination of fission fragments and emitted prompt neutron characteristics", in Proc. of the Int. Workshop on Nuclear Fission and Fission Product Spectroscopy, Cadarache (France), May 11-14, 2005. Eds. G. Fioni et al., AIP Conf. Proc., vol. 447, p. 369.

[301] H. R. Faust, Z. Bao, "Higher moments in the kinetic energy distributions of fission fragments", NuCL. PHYs. A 736, 55 (2004).

[302] Ch. Straede, C. Budtz-Joergensen, and H.-H. Knitter, ${ }^{2} 235 \mathrm{U}(\mathrm{n}, \mathrm{f})$ fragment mass-, kinetic energy- and angular distributions for incident neutron energies between thermal and $6 \mathrm{MeV} "$, Nucl. Phys. A 462, 85 (1987).

[303] J. W. Meadows, C. Budtz-Joergensen, "The fission fragment angular distributions and total kinetic energies for ${ }^{235} \mathrm{U}(\mathrm{n}, \mathrm{f})$ from 0.18 to $8.83 \mathrm{MeV} "$, Nucl. Data \& Meas. Series, ANL/NDM-64 (1963).

[304] J.-C. Benoit, "Développement dun code de propagation des incertitudes des données nucléaires sur la puissance résiduelle dans les réacteurs à neutrons rapides", $\mathrm{PhD}$ thesis, Université Paris Sud, 2012 (in French).

[305] R. A. Forrest, "FISPACT2007: User manual", March 2007.
[306] A. Tobias, "Derivation of decay heat benchmarks for ${ }^{235} \mathrm{U}$ and ${ }^{239} \mathrm{Pu}$ by a least squares fit to measured data", CEGB Report RD/B/6210/R89, May 1989.

[307] M. A. Kellet, O. Bersillon, and R. W. Mills, "The JEFF3.1/-3.1.1 radioactive decay data and fission yields sublibraries", JEFF Report 20, NEA Data Bank of the OECD, 2009.

[308] M.-L. Giacri-Mauborgne, "Création d'une bibliothèque d'activation photo-nucléaire et mesures de spectres d'émission de neutrons retardés", $\mathrm{PhD}$ thesis, University Caen, 2005 (in French).

[309] G. Rudstam, P. H. Finck, A. Filip, A. D'Angelo, and R. D. McKnight, "Delayed neutron data for the major actinides", NEA/WPEC6 and references therein.

[310] G. R. Keepin, T. F. Wimett, and R. K. Zeigler, "Delayed neutrons from fissionable isotopes of uranium, plutonium, and thorium", Phys. Rev. 107, 1044 (1957).

[311] D. R. Alexander and M. S. Krick, "Delayed neutron yield calculations for the neutron-induced fission of uranium-235 as a function of the incident neutron energy", NuCl. SCI. ENG. 62, 627 (1977).

[312] J. van Aarle, W. Westmeier, R. A. Esterlund and P. Patzelt, " ${ }^{252} \mathrm{Cf}$ : neutron multiplicities in correlation with fission-fragment mass and energy", NuCL. PHYs. A 578, 77 (1994).

[313] F.-J. Hambsch, G. Vladuca, A. Tudora, S. Oberstedt, and I. Ruskov, "Prompt fission neutron spectrum evaluation for ${ }^{252} \mathrm{Cf}(\mathrm{SF})$ in the frame of the multi-modal fission model", Ann. Nucl. Energy 32, 1032 (2005). 